\title{
Correlation functions in Schwarzian liquid
}

\author{
Yong-Hui Qi, ${ }^{1,2, *}$ Yunseok Seo, ${ }^{1,3,}$ Sang-Jin Sin, ${ }^{1, *}$ and Geunho Song ${ }^{1, \S}$ \\ ${ }^{1}$ Department of Physics, Hanyang University, Seoul, 04763, Korea \\ ${ }^{2}$ Center for High Energy Physics, Peking University, Beijing, 100871, Peoples Republic of China \\ ${ }^{3}$ GIST College, Gwangju Institute of Science and Technology, Gwangju 500-712, Korea
}

(Received 24 April 2018; published 5 March 2019)

\begin{abstract}
We analytically study low-temperature universal properties of a class of SYK-type models in the large N limit from the $\mathrm{AdS}_{2}$ gravity dual side in terms of $\mathrm{SL}(2, R)$-invariant Schwarzian action. The quantum correction to the conformal field theory $\mathrm{CFT}_{1}$ two-point correlation function due to the Schwarzian action produces a transfer of degree of freedom from the quasiparticle peak to the Hubbard band in density of states (DOS), a signature strong correlation. In Schwinger-Keldysh (SK) formalism, we calculate higherpoint thermal out-of-time order correlation (OTOC) functions, which indicate quantum chaos by having a Lyapunov exponent. Higher-order local spin-spin correlations are also calculated, which can be related to the dynamical local susceptibility of quantum liquids such as the spin liquid in disordered metals, marginal Fermi liquid, non-Fermi liquid, etc.
\end{abstract}

DOI: 10.1103/PhysRevD.99.066001

\section{INTRODUCTION}

Recently, the Sachdev-Ye-Kitaev (SYK) model [1,2] attracted a lot of interest [3-37]. It is a $(0+1)$-dimensional quantum mechanical system composed of $N$ Majorana fermions, with a random, all-to-all quartic interaction.

There are three novel features of the SYK model. The first one is the solvability at large $\mathrm{N}$ in the strong coupling limit. The second one is the emergence of conformal symmetry at the IR limit, as well as its spontaneous breaking which results in soft modes as the pseudo Nambu-Goldstone bosons (pNGBs) $[7,23,33]$. The third one is the quantum chaos behavior in four-point correlation functions.

There are several ways to generalize the SYK model. One is the generalization in flavor symmetry, which generalize the four-Majorana fermion interactions of SYK-like model to $q_{f}$-fermion with $f$-flavors [12]. The other is its generalization in higher-dimensional spacetime $[11,13,22]$. Since in the $(0+1)$-dimensional SYK model, the Majorana field $\psi$ is dimensionless $([\psi]=0)$, the coupling $J$ is always dimensionful $([J]=1)$. Thus, the $p \geq 4$-fermion interaction is always relevant at UV. While in general this is not true in $D \geq 2$, since the UV relevance of the $p$-fermions

\footnotetext{
„yhqi@pku.edu.cn

yseo@gist.ac.kr

*sjsin@ hanyang.ac.kr

§sgh8774@gmail.com
}

Published by the American Physical Society under the terms of the Creative Commons Attribution 4.0 International license. Further distribution of this work must maintain attribution to the author(s) and the published article's title, journal citation, and DOI. Funded by SCOAP. interactions depends on the dimension of the spacetime. For example, in a generalization of the $(1+1)$-dimensional SYK model, in analogy to the two-dimensional GrossNeveu model, it can be obtained by integrating out the tensor field that coupled with a new vector field [24]. In this case, the fermion field is dimensionful $([\psi]=1 / 2)$, meanwhile the coupling is dimensionless $([J]=0)$. Thus, the fourfermion interaction term is marginal. Generally speaking, the generalization to higher dimensions $(D \geq 3)$ will inevitably lead to irrelevant $p$-fermion interactions.

In this paper, motivated by the novel features of the spin liquid phase in SY state [2,38] in disordered metals depicted by SYK-like models [39-41], we study a general class of strongly interacting $(0+1)$-dimensional quantum mechanical models in the large $\mathrm{N}$ limit [16], whose lowenergy dynamics is depicted by the Schwarzian action. The action leads to pNGBs modes, which results in quantum corrections to the correlations of the quantum liquid such as "marginal Fermi liquid" (MFL) [42] and "fractionalized Fermi liquid" (FFL) [43].

The Schwarzian action is determined by the pattern of spontaneous breaking of reparametrization symmetry [7]. It has been conjectured that the gravity dual of the SYK model can be described by a two-dimensional dilaton gravity [6]: one example is the Jackiw-Teitelboim (JT) model $[44,45]$. Another example is the Almheiri-Polchinski (AP) model $[8,46]$. The low-energy quantum description of the SYK model is proposed to be holographically dual to a $(1+1)$-dimensional model of a black hole [5,23], although it is not completely conventional AdS/CFT.

By taking accounting of the soft mode from Schwarzian effective action, we set up a relatively simple reductive field 
method to calculate higher-point quantum correlation functions, order by order at large $\mathrm{N}$. With the matter correlation functions, we are able to calculate the spin-spin correlation functions and retarded Greens' functions. As it will be shown, the Schwarzian correction to the $\mathrm{AdS}_{2}$ symmetry, leads to a Hubbard band in the spectral function by transferring the degree of freedoms from the quasiparticle peak at CFT $_{1}$ fixed point to the side band, a hallmark of the strongly interacting systems. Our analytical results are consistent with the general tendency of the modification of the spectral functions by interaction, by computing in density functional theory (DFT) [47], e.g., the DOS result as Fig. 4B in Ref. [48], or in the dynamical mean-field theory (DMFT) approach [49].

The identification of the gravity dual of the SYK model is not exact. However, in strongly interacting system, the explicit model dependence is easily washed out. Namely, if two models have the same symmetry, the IR dynamics is controlled only by universal features. Whatever gravity dual model we take, the low-energy result is the same and controlled by the Schwarzian action. This is why we believe that our calculation is relevant to SYK model.

We are not calculating the SYK model, which is about the fermions with random coupling, while we are dealing with the correlation function of bosonic operators without it. However, it is rather interesting to observe that the resulting two- and four-point correlation functions are almost the same (apart from the sign factors) with the known results of SYK models [5] by field theory calculations.

We think the reason is as follow: in $(0+1)$ dimension there is no spinor and spin connection, and there is not much difference between the fundamental field and the composite boson apart from the scaling dimension and antisymmetry coming from the anticommutativity of fermion operators. So it is not surprising that the gravity calculation which pick up the leading order in large $\mathrm{N}$ should give the same correlation function if we can set the scaling dimension arbitrary.

The paper is organized as follow. In Sec. II, we study the correlation functions from Schwarzian action at both zero and finite temperature. In Sec. III, we study the zero temperature and thermal retarded Green's functions with loop correction from pNGBs, and local dynamical susceptibility of quantum liquid. The higher-order local spin-spin correlation functions beyond local susceptibility are also investigated. Generalization of $\mathrm{AdS}_{2}$ spacetime as near IR horizon of RN black hole in $\mathrm{AdS}_{d+1}$ spacetime is studied in Appendix C. In Sec. IV, we study higher-point correlation functions, the thermal OTOC functions in SK formalism.

\section{CORRELATION FUNCTIONS FROM SCHWARZIAN}

In this section, based on the low-energy effective action of the Schwarzian theory of time reparametrization from two-dimensional gravity, we calculate the correlation functions, especially the two-point one [50].

\section{A. Effective action of gravity and soft mode}

In the linearized theory of the boundary action of two-dimensional gravity, i.e., one-dimensional effective action as

$$
S_{\mathrm{eff}}=-C_{g} \int d t \phi_{r}(t) \operatorname{Sch}(f(t), t)
$$

$C_{g}$ is a constant depending on the bulk gravity parameter, $t$ is the boundary time coordinate, $f(t)$ is the field variable, $\phi_{r}(t)$ is the normalizable part of the dilaton, which is a constant on the cutoff boundary and plays a role of external coupling, while the divergent part that blows up at boundary is absorbed by a counter term, and can be identified as the source in nearly- $\mathrm{AdS}_{2}\left(\mathrm{NAdS}_{2}\right) /$ nearly$\mathrm{CFT}_{1}\left(\mathrm{NCFT}_{1}\right)$ description, $\operatorname{Sch}(f, t)$ is the Schwarzian derivative defined as

$\operatorname{Sch}(f, t)=\left(\frac{f^{\prime \prime}}{f^{\prime}}\right)^{\prime}-\frac{1}{2}\left(\frac{f^{\prime \prime}}{f^{\prime}}\right)^{2}=\frac{f^{\prime \prime \prime}}{f^{\prime}}-\frac{3}{2}\left(\frac{f^{\prime \prime}}{f^{\prime}}\right)^{2}$,

where the prime ' denotes the derivative with respect to $t$. The zero modes is described by the Schwarzian action. The effective action has a global $\operatorname{SL}(2)$ invariance, which is obvious that by noticing $\operatorname{Sch}((a f+b) /(c f+d), t)=$ $\operatorname{Sch}(f, t)$. By doing variation with respect to $f(t)$, the action becomes

$$
\delta S_{\mathrm{eff}} \sim-C_{g} \int \phi_{r}(t) d t \frac{[\operatorname{Sch}(f(t), t)]^{\prime}}{f^{\prime}} \delta f,
$$

and by using the property as

$$
\frac{[\operatorname{Sch}(f, t)]^{\prime}}{f^{\prime}}=\left[\frac{1}{f^{\prime}}\left(\frac{\left(f^{\prime}\right)^{\prime}}{f^{\prime}}\right)^{\prime}\right]^{\prime},
$$

and that $\delta\left(f^{\prime}\right)^{-1}=-\left(f^{\prime}\right)^{-2} \delta f^{\prime}$, one obtains the field equation of motion with respect to $t(s)$ turns out to be

$$
\left[\frac{1}{f^{\prime}}\left(\frac{\left(f^{\prime} \phi_{r}\right)^{\prime}}{f^{\prime}}\right)\right]^{\prime}=0
$$

which becomes $[\operatorname{Sch}(f, t)]^{\prime} / t^{\prime}=0$ when $\phi_{r}$ is a constant. One of the simplest nontrivial solutions might be a nonconstant function with constant Schwarzian.

\section{Zero temperature soft mode propagator}

Consider a linear transformation $f(\tau)=\tau(t)$, then according to the composition rule of Schwarzian derivative as

$$
\operatorname{Sch}(g(f), t)=f^{\prime 2} \operatorname{Sch}(g(f), f)+\operatorname{Sch}(f, t),
$$

where $g(f)=g(f(t))$ and $f=f(t)$, one has $\operatorname{Sch}(f, t)=$ $\tau^{\prime 2} \operatorname{Sch}(f, \tau)+\operatorname{Sch}(\tau, t)$, where $\operatorname{Sch}(f, \tau)=1 / 2$, when $\tau$ is 
a linear function of $t, \operatorname{Sch}(t, s)$ is constant and satisfies the equation of motion of the Schwarzian action, i.e., $\operatorname{Sch}(\tau, t)^{\prime} / f^{\prime}=0$. In the perturbative approach, one can set

$$
\tau(t) \equiv t+\epsilon k(t)
$$

where $t=i t$ is imaginary time and $\epsilon \ll 1$ is the expansion parameter, which can be chosen as the bulk gravitational interaction coupling, i.e., $\epsilon=\kappa_{N} \sim \sqrt{G_{N}}$, which is proportional to $G_{N}^{1 / 2}$ in gravity or large $N^{-1 / 2}$ as in the SYK model. By expanding the Schwarzian action, one has

$\operatorname{Sch}(f, t)=\epsilon k^{\prime \prime \prime}+\epsilon^{2}\left(-\frac{3}{2} k^{\prime \prime 2}-\left(k^{\prime \prime} k^{\prime}\right)^{\prime}\right)+O\left(\epsilon^{3}\right)$.

By dropping total derivative term, the leading-order action at $\epsilon^{2}$ order reads as

$$
S_{\mathrm{eff}}=\frac{3 C}{2} \epsilon^{2} \int d t k^{\prime \prime 2},
$$

where $C \propto \bar{\phi}_{r}$. By doing a Fourier transformation $k(t)=\sum_{n} k_{n} e^{\text {int }}$, the action becomes

$$
S_{\text {eff }}=\frac{3 C}{2} \int d t n^{4} k_{n} k_{-n},
$$

where hence and forth, the Einstein summation notation convention is applied for repeated $n \in \mathbb{Z}$. Thus, the soft mode propagator can be obtained

$$
\begin{aligned}
\epsilon^{2}\left\langle k\left(t_{1}\right) k\left(t_{2}\right)\right\rangle & =\frac{1}{6 \pi C} \sum_{n \neq 0} \frac{e^{\text {int }}}{n^{4}} \\
& =\frac{1}{6 \pi C}\left(\frac{1}{24}(|t|-\pi)^{4}-\frac{\pi^{2}}{12}(|t|-\pi)^{2}+\frac{7 \pi^{4}}{360}\right), \\
\epsilon^{2}\left\langle k\left(t_{1}\right) k^{\prime}\left(t_{2}\right)\right\rangle & =\frac{1}{36 \pi C} \operatorname{sgn}(t)(\pi-|t|)(2 \pi-|t|)|t|, \\
\epsilon^{2}\left\langle k^{\prime}\left(t_{1}\right) k^{\prime}\left(t_{2}\right)\right\rangle & =\frac{1}{6 \pi C}\left(-\frac{\pi^{2}}{6}+\frac{1}{2}(\pi-|t|)^{2}\right),
\end{aligned}
$$

where $t \equiv t_{1}-t_{2}$ and we have used that

$$
\begin{aligned}
\mathrm{Li}_{4}(z)+\mathrm{Li}_{4}\left(\frac{1}{z}\right) & =-\frac{1}{4 !}(2 \pi i)^{4} B_{4}\left(1+\frac{i}{2 \pi} \ln z\right) \\
& =-\frac{z^{4}}{24}+\frac{\pi z^{3}}{6}-\frac{\pi^{2} z^{2}}{6}+\frac{\pi^{4}}{45},
\end{aligned}
$$

where $z=e^{i t} . \operatorname{Li}_{n}(x)$ is the polylogarithmic function defined by the series $\operatorname{Li}_{n}(x)=\sum_{k=1}^{n} z^{k} / k^{n}$ for $|z|<1$ and $B_{n}(x)$ is the Bernoulli polynomial.

\section{Thermal soft mode propagator}

Consider a thermal circle transformation $f(\tau)=$ $\tan (\pi \tau / \beta)$ satisfying $f(\tau+\beta)=f(\tau)$ with period length $\beta=2 \pi$, in other words, by imposing a mapping from

$$
f(t)=\tan \frac{\tau(t)}{2}, \quad \tau(t)=i \frac{2 \pi}{\beta} t \equiv i t
$$

then the composition rule of Schwarzian derivative in Eq. (2.6) leads to an action results as $\operatorname{Sch}(f, t)=\tau^{\prime 2} / 2+$ $\operatorname{Sch}(\tau, t)$, when $\tau$ is a linear function of $t, \operatorname{Sch}(t, s)$ is constant and satisfies the equation of motion of the Schwarzian action, i.e., $\operatorname{Sch}(f, t)^{\prime} / f^{\prime}=0$. This can be traced back to the bulk equation of motion, which gives the dilaton solution.

In the perturbative approach as in Eq. (2.7), after expanding the effective Schwarzian action, higher-order self-interaction terms for $k(\tau(t))$ are present, which is suppressed by factor of $\epsilon$. By expanding the Schwarzian action, one has

$$
\begin{aligned}
\operatorname{Sch}(f, t)= & \frac{1}{2}+\epsilon\left(k^{\prime}+k^{\prime \prime \prime}\right)+\epsilon^{2}\left(\frac{1}{2} k^{\prime 2}-\frac{1}{2} k^{\prime \prime 2}-\left(k^{\prime \prime} k^{\prime}\right)^{\prime}\right) \\
& +O\left(\epsilon^{3}\right) .
\end{aligned}
$$

By dropping total derivative term, the leading-order action at $\epsilon^{2}$ order reads as

$$
S_{\text {eff }}=\frac{C}{2} \epsilon^{2} \int d t\left(k^{\prime \prime 2}-k^{\prime 2}\right)
$$

where $C \propto C_{g} \bar{\phi}_{r}$. By doing a Fourier transformation as $k(t)=\sum_{n} k_{n} e^{\text {int }}$ where $t \in[0,2 \pi]$, the action becomes

$$
S_{\mathrm{eff}}=\frac{C}{2} \epsilon^{2} \int d t\left(n^{4}-n^{2}\right) k_{n} k_{-n},
$$

where for repeated $n$ the Einstein summation convention is applied as stated before. Thus, the soft mode propagator can be obtained as

$$
\begin{aligned}
\epsilon^{2}\left\langle k\left(t_{1}\right) k\left(t_{2}\right)\right\rangle= & \frac{1}{2 \pi C} \sum_{n \neq 0, \pm 1} \frac{e^{\mathrm{int}}}{n^{2}\left(n^{2}-1\right)} \\
= & \frac{1}{2 \pi C}\left(1+\frac{\pi^{2}}{6}-\frac{(|t|-\pi)^{2}}{2}\right. \\
& \left.+(|t|-\pi) \sin |t|+\frac{5}{2} \cos |t|\right), \\
\epsilon^{2}\left\langle k\left(t_{1}\right) k^{\prime}\left(t_{2}\right)\right\rangle= & \frac{\operatorname{sgn}(t)}{2 \pi C}\left((\pi-|t|)(1-\cos |t|)-\frac{3}{2} \sin |t|\right), \\
\epsilon^{2}\left\langle k^{\prime}\left(t_{1}\right) k^{\prime}\left(t_{2}\right)\right\rangle= & \frac{1}{2 \pi C}\left(1+\frac{1}{2} \cos |t|-(\pi-|t|) \sin |t|\right),
\end{aligned}
$$

where $t \equiv t_{1}-t_{2}$ and we have used that 


$$
\begin{aligned}
\mathrm{Li}_{2}(z)+\mathrm{Li}_{2}\left(\frac{1}{z}\right) & =-\frac{1}{2}(2 \pi i)^{2} B_{2}\left(1+\frac{i}{2 \pi} \ln z\right) \\
& =\frac{\pi^{2}}{3}-\pi z+\frac{z^{2}}{2},
\end{aligned}
$$

where $z=e^{i t}$.

\section{B. Effective action of matter}

The $n$-point function of a matter field, e.g., a scalar $\Phi$ in $\mathrm{NAdS}_{2}$, can be computed by coupling the matter field to the bulk gravity in $\mathrm{AdS}_{2}$, and then rewriting the action by using $f(t)$, and rescaling by a factor $f^{\prime}(t)^{\Delta}$ at the insertion of each operator.

\section{Two-point matter correlation functions}

For a massive scalar $\Phi$ in $\mathrm{AdS}_{2}$ spacetime in Poincaré coordinate, since all gravitational configurations in twodimensional spacetime can be described by the metric, and the effective action is [7]

$$
\begin{aligned}
S_{\chi} & =\frac{1}{2} \int d^{2} x \sqrt{-g}\left[(\nabla \Phi)^{2}+m^{2} \Phi^{2}\right] \\
& =-N \int d t d t^{\prime} \frac{\Phi_{0}(t) \Phi_{0}\left(t^{\prime}\right)}{\left|t-t^{\prime}\right|^{2 \Delta}}+\cdots,
\end{aligned}
$$

where $N=\nu C_{\Delta}=(\Delta-1 / 2) \Gamma[\Delta] /\left[\sqrt{\pi} \Gamma\left(\Delta-\frac{1}{2}\right)\right]$ and we have used the asymptotic behavior of $\Phi$ at boundary

$$
\begin{aligned}
\Phi(t, z) & =\int d t^{\prime} K_{\Delta}\left(z, t, t^{\prime}\right) \Phi_{0}\left(t^{\prime}\right) \\
& =\left(t^{\prime}\right)^{1-\Delta} \Phi_{0}(t)+\cdots, \quad z \rightarrow 0,
\end{aligned}
$$

where $t^{\prime} \equiv z / \epsilon, K_{0}\left(z, t, t^{\prime}\right)$ is the normalized bulk-toboundary Green's function for $\Delta>1 / 2$ as

$$
K_{\Delta}\left(z, t, t^{\prime}\right)=C_{\Delta}\left(\frac{z}{z^{2}+\left(t-t^{\prime}\right)^{2}}\right)^{\Delta},
$$

where $C_{\Delta}=\Gamma(\Delta) /[\sqrt{\pi} \Gamma(\Delta-1 / 2)], \Phi_{0}(t)$ can be viewed as a source for a scalar operator with conformal dimension $\Delta$, e.g., for free scalar in pure $\mathrm{AdS}_{2} / \mathrm{CFT}_{1}$ case, $\Delta=\Delta_{+}$is the largest root of indicial equation with $\Delta_{ \pm}=1 / 2 \pm$ $\sqrt{1 / 4+m^{2}} \geq 1$. Consider that the trajectory of the boundary curve is $f(t)$, which can be transformed to the desired boundary conditions as

$$
\Phi_{0}(t)=\left[f^{\prime}(t)\right]^{1-\Delta} \Phi_{0}(f(t)) .
$$

Then, the effective action can be reparametrized as

$$
\bar{S}_{\text {eff }}=-N \int d t d t^{\prime}\left(\frac{f^{\prime}(t) f^{\prime}\left(t^{\prime}\right)}{\left[f(t)-f\left(t^{\prime}\right)\right]^{2}}\right)^{\Delta} \Phi_{0}(t) \Phi_{0}\left(t^{\prime}\right) .
$$

The two-point function of the dual field $\mathcal{O}(t)$ to the source $\Phi_{0}$ can be read as

$$
G\left(t, t^{\prime}\right) \equiv\left\langle\mathcal{O}(t) \mathcal{O}\left(t^{\prime}\right)\right\rangle \sim\left(\frac{f^{\prime}(t) f^{\prime}\left(t^{\prime}\right)}{\left[f(t)-f\left(t^{\prime}\right)\right]^{2}}\right)^{\Delta} .
$$

For the convenience of generalization to the higher-point correlation function, let's relabel $t=t_{1}, t^{\prime}=t_{2}$, then the two-point function can be reexpressed in a more tidy form as

$$
G_{2}\left(t_{1}, t_{2}\right)=2 N\left(\frac{f_{1}^{\prime} f_{2}^{\prime}}{f_{12}^{2}}\right)^{\Delta}
$$

where we have made the new notations as $f_{i}=f\left(t_{i}\right)$ and $f_{12}=\left|f\left(t_{1}\right)-f\left(t_{2}\right)\right|$.

\section{Three-point matter correlation functions}

In addition to the quadratic interactions in Eq. (2.18), three-point matter correlation functions can be obtained by introducing the cubic interaction term as [51]

$$
S_{\mathrm{int}}=\sum_{n \geq 3} \frac{\lambda_{n}}{n} \Phi^{n}=\frac{\lambda_{3}}{3} \Phi^{3}+\cdots,
$$

where $\cdots$ denotes the quartic and higher-order interactions. In this case, the equation of motion becomes

$$
\left(\nabla^{2}-m^{2}\right) \Phi=\lambda_{3} \Phi^{2}+\cdots
$$

In this case, the solution for $\Phi$ turns out to be

$$
\begin{aligned}
\Phi(t, z)= & \int d t^{\prime} K_{\Delta}\left(t, z ; t^{\prime}\right) \Phi_{0}\left(t^{\prime}\right)+\int d t^{\prime} d z^{\prime} G\left(t, z ; t^{\prime}, z^{\prime}\right) \\
& \times \lambda_{3} \int \prod_{m=1}^{2} d t_{m} K_{\Delta}\left(t^{\prime}, z^{\prime}, t_{m}\right) \Phi_{0}\left(t_{m}\right)
\end{aligned}
$$

where $K_{\Delta}\left(t, z ; t^{\prime}\right)$ is the boundary-to-bulk propagator in Eq. (2.20) and $G\left(t, z ; t^{\prime}, z^{\prime}\right)$ is the bulk-to-bulk Green's function which can be expressed in terms of Hypergeometric function as

$$
G\left(t, z ; t^{\prime}, z^{\prime}\right)=\frac{2 C_{\Delta}}{\nu}\left(\frac{s}{2}\right){ }_{2}^{\Delta} F_{1}\left(\frac{\Delta}{2}, \frac{\Delta}{2}+\frac{1}{2} ; \nu+1 ; s^{2}\right),
$$

where

$$
\Delta=\Delta_{+}, \quad \nu=\Delta-\frac{1}{2}, \quad s=\frac{2 z z^{\prime}}{z^{2}+z^{\prime 2}+\left(t-t^{\prime}\right)^{2}} .
$$

In the $s \rightarrow 0$ limit, one has $G\left(t, z ; t^{\prime}, z^{\prime}\right)=\left(2 C_{\Delta} / \nu\right)(s / 2)^{\Delta}$. By substituting the classical solution in Eq. (2.27) back into 
the original action, integrating by parts and using the properties of the bulk Green's function, one obtains the effective action as

$$
I_{\mathrm{int}}^{\mathrm{eff}}=\lambda_{3} \int \prod_{i=1}^{3} d t_{i} \Phi_{0}\left(t_{i}\right) I_{3}\left(t_{1}, t_{2}, t_{3}\right)+\cdots
$$

where

$$
I_{3}\left(t_{1}, t_{2}, t_{3}\right) \equiv \int d t \frac{d z}{z^{2}} \prod_{j=1}^{3} K_{\Delta}\left(t, z ; t_{j}\right) .
$$

Then the generic connected part of the tree-level three-point functions from the effective action becomes

$$
\left\langle\mathcal{O}\left(t_{1}\right) \mathcal{O}\left(t_{2}\right) \mathcal{O}\left(t_{3}\right)\right\rangle=-\lambda_{3} I_{3}\left(t_{1}, t_{2}, t_{3}\right) .
$$

To be concrete, one obtains

$$
\left\langle\mathcal{O}\left(t_{1}\right) \mathcal{O}\left(t_{2}\right) \mathcal{O}\left(t_{3}\right)\right\rangle=\frac{C_{3}}{\left(t_{12} t_{13} t_{23}\right)^{\Delta}}
$$

where $C_{3}=-\lambda_{3} \Gamma(\Delta / 2)^{3} \Gamma((3 \Delta-1) / 2) / 2 \pi \Gamma(\Delta-1 / 2)^{3}$. In analogy to Eq. (2.23), after the time-reparametrization transformation, the three-point functions of the boundary dual field $\mathcal{O}(t)$ to the source can be read as

$G_{3}\left(t_{1}, t_{2}, t_{3}\right) \equiv\left\langle\mathcal{O}\left(t_{1}\right) \mathcal{O}\left(t_{2}\right) \mathcal{O}\left(t_{3}\right)\right\rangle \sim\left(\frac{f_{1}^{\prime} f_{2}^{\prime} f_{3}^{\prime}}{f_{12} f_{13} f_{23}}\right)^{\Delta}$,

where we have made the abbreviation for the notations $f_{i} \equiv$ $f\left(t_{i}\right)$ and $f_{i j} \equiv f\left(t_{i}\right)-f\left(t_{j}\right)$.

\section{Zero temperature correlation functions}

Consider expand the boundary $t$ around the saddle of imaginary time $\tau$ as

$$
f(t)=t+\epsilon k(t) .
$$

The two-point function of the dual operator can be expanded as

$$
G\left(t_{1}, t_{2}\right)=\frac{1+\epsilon \mathcal{B}_{1}\left(t_{12}\right)+\epsilon^{2} \mathcal{B}_{2}\left(t_{12}\right)+O\left(\epsilon^{3}\right)}{t_{12}^{2 \Delta}},
$$

where $\mathcal{B}_{n}\left(t_{12}\right) \equiv \mathcal{B}_{n}\left(t_{1}-t_{2}\right)$ with $n=1,2, \ldots$ and

$$
\begin{aligned}
\mathcal{B}_{1}\left(t_{12}\right) & =\Delta\left(k^{\prime}\left(t_{1}\right)+k^{\prime}\left(t_{2}\right)-2 \frac{k\left(t_{1}\right)-k\left(t_{2}\right)}{t_{12}}\right), \\
\mathcal{B}_{2}\left(t_{12}\right) & =\Delta\left(\frac{\left(k\left(t_{1}\right)-k\left(t_{2}\right)\right)^{2}}{t_{12}^{2}}-\frac{k^{\prime}\left(t_{1}\right)^{2}+k^{\prime}\left(t_{2}\right)^{2}}{2}\right) \\
& +2 \Delta^{2}\left(\frac{k^{\prime}\left(t_{1}\right)+k^{\prime}\left(t_{2}\right)}{2}-\frac{k\left(t_{1}\right)-k\left(t_{2}\right)}{t_{12}}\right)^{2},
\end{aligned}
$$

where $t_{12} \equiv t_{1}-t_{2}$. In analogy to the finite temperature case, the two-, four-, six- and eight-point functions are, respectively, given by

$$
\begin{aligned}
G_{2} & =N \frac{1+\epsilon^{2}\left\langle\mathcal{B}_{2}\left(t_{12}\right)\right\rangle}{t_{12}^{2 \Delta}}, \\
G_{4} & =N \frac{\epsilon^{2}\left\langle: \mathcal{B}_{1}\left(t_{12}\right) \mathcal{B}_{1}\left(t_{34}\right):\right\rangle}{t_{12}^{2 \Delta} t_{34}^{2 \Delta}}, \\
G_{6} & =N \frac{\epsilon^{4}\left\langle: \mathcal{B}_{1}\left(t_{12}\right) \mathcal{B}_{1}\left(t_{34}\right) \mathcal{B}_{2}\left(t_{56}\right):\right\rangle}{t_{12}^{2 \Delta} t_{34}^{2 \Delta} t_{56}^{2 \Delta}}, \\
G_{8} & =N \frac{\epsilon^{4}\left\langle: \mathcal{B}_{1}\left(t_{12}\right) \mathcal{B}_{1}\left(t_{34}\right) \mathcal{B}_{1}\left(t_{56}\right) \mathcal{B}_{1}\left(t_{78}\right):\right\rangle}{t_{12}^{2 \Delta} t_{34}^{2 \Delta} t_{56}^{2 \Delta} t_{78}^{2 \Delta}},
\end{aligned}
$$

where $G_{2}=G_{2}\left(t_{1}, t_{2}\right), G_{4}\left(t_{1}, t_{2}, t_{3}, t_{4}\right)$, etc.

\section{Thermal correlation functions}

Consider expand the boundary $t$ around the saddle of a thermal circle, according to Eqs. (2.13) and (2.7) as

$$
f(t)=\tan \frac{t+\epsilon k(t)}{2}, \quad \text { or } \quad f(t)=e^{i t+i \epsilon k(t)},
$$

where we have dropped a common factor $2 \pi / \beta$, so to recover one has to rescale $t \rightarrow 2 \pi / \beta t$. The two-point function of the dual operators can be expanded as

$$
\begin{aligned}
G\left(t_{1}, t_{2}\right) & =\frac{1+\epsilon \mathcal{B}_{1}\left(t_{12}\right)+\epsilon^{2} \mathcal{B}_{2}\left(t_{12}\right)+O\left(\epsilon^{3}\right)}{\left(2 \sin \frac{t_{12}}{2}\right)^{2 \Delta}}, \\
& =G_{0}\left(t_{1}, t_{2}\right)\left[1+\epsilon \mathcal{B}_{1}\left(t_{12}\right)+\epsilon^{2} \mathcal{B}_{2}\left(t_{12}\right)+O\left(\epsilon^{3}\right)\right],
\end{aligned}
$$

where $\mathcal{B}_{n}\left(t_{i j}\right) \equiv \mathcal{B}_{n}\left(t_{i}, t_{j}\right)$. By neglecting the perturbation expansion term, $\epsilon \rightarrow 0$, the $\mathrm{AdS}_{2}$ thermal two-point functions is recovered as

$$
G_{0}\left(t_{1}, t_{2}\right)=\frac{1}{\left(2 \sin \frac{t_{12}}{2}\right)^{2 \Delta}}=\left(\frac{\pi}{\beta \sin \frac{\pi t_{12}}{\beta}}\right)^{2 \Delta},
$$

where in the last equality, we have recovered the thermal factor $t_{12} \rightarrow(2 \pi) / \beta t_{12}$. The leading correction to the thermal two-point-Green's function can be expressed more explicitly as 


$$
\begin{aligned}
\mathcal{B}_{1}\left(t_{12}\right)= & \Delta\left(k^{\prime}\left(t_{1}\right)+k^{\prime}\left(t_{2}\right)-\frac{k\left(t_{1}\right)-k\left(t_{2}\right)}{\tan \frac{t_{12}}{2}}\right), \\
\mathcal{B}_{2}\left(t_{12}\right)= & \frac{1}{2} \Delta^{2}\left(k^{\prime}\left(t_{1}\right)+k^{\prime}\left(t_{2}\right)+\frac{k\left(t_{2}\right)-k\left(t_{1}\right)}{\tan \frac{t_{12}}{2}}\right)^{2} \\
& +\frac{1}{4} \Delta\left(\frac{\left[k\left(t_{1}\right)-k\left(t_{2}\right)\right]^{2}}{\left(\sin \frac{t_{12}}{2}\right)^{2}}-2\left[k^{\prime}\left(t_{1}\right)^{2}+k^{\prime}\left(t_{2}\right)^{2}\right]\right),
\end{aligned}
$$

where for the brief ness, we do not recover the thermal factor. The expansion can be viewed in Feynman diagrams as shown in Fig. 1. It will be obvious in the following that the higher order with $\epsilon^{n} \mathcal{B}_{n}\left(t_{1}, t_{2}\right)$ for $n \geq 3$ will not contributes to the leading order of all point functions.

The generating functional of connected correlators can be expanded as

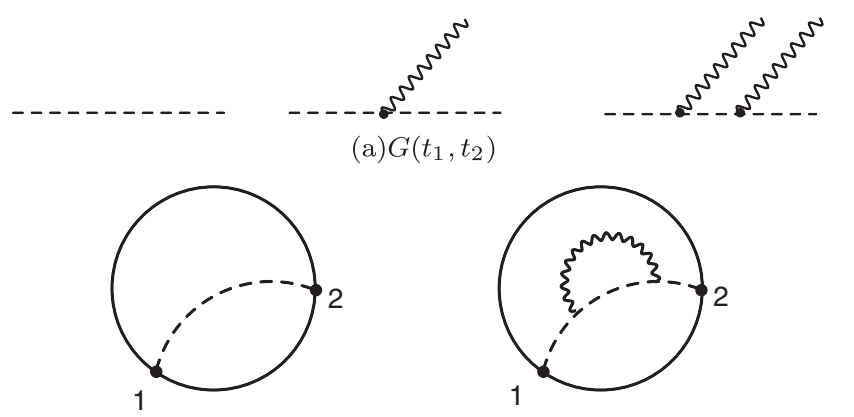

(b) $G_{2}\left(t_{1}, t_{2}\right)$

FIG. 1. (a) Feynman rules for two-point functions of scalars (dashed line) $G\left(t_{1}, t_{2}\right)$ and the corrections from gravitational soft mode (double wave lines) for $\mathcal{B}_{1}\left(t_{12}\right)$ and $\mathcal{B}_{2}\left(t_{12}\right)$ as shown in Eq. (2.42). (b) Feynman diagrams for two-point correlation functions $G_{2}\left(t_{1}, t_{2}\right)$ of scalar fields with loop corrections from soft modes as shown in Eq. (2.17).

$$
\begin{aligned}
& W=\ln \langle Z\rangle=\ln \left\langle e^{-S_{\text {eff }}}\right\rangle=\ln \left[\int \mathcal { D } \mu [ f ] e ^ { - \overline { S } _ { 0 } } \left(1+\epsilon^{2} N \int \prod_{i=1}^{2} d t_{i} \Phi_{0}\left(t_{i}\right) \frac{\mathcal{B}_{2}\left(t_{12}\right)+\epsilon^{2} \mathcal{B}_{4}\left(t_{12}\right)}{\left(2 \sin \frac{t_{12}}{2}\right)^{2 \Delta}}\right.\right. \\
& +\frac{1}{2} \epsilon^{2} \int \prod_{i=1}^{4} d t_{i} \Phi_{0}\left(t_{i}\right) \frac{: \mathcal{B}_{1}\left(t_{12}\right) \mathcal{B}_{1}\left(t_{34}\right):+\epsilon^{2}\left(: \mathcal{B}_{2}\left(t_{12}\right) \mathcal{B}_{2}\left(t_{34}\right):+2: \mathcal{B}_{1}\left(t_{12}\right) \mathcal{B}_{3}\left(t_{34}\right):\right)}{\left(2 \sin \frac{t_{12}}{2}\right)^{2 \Delta}\left(2 \sin \frac{t_{34}}{2}\right)^{2 \Delta}} \\
& +\frac{1}{6} \epsilon^{4} \int \prod_{i=1}^{6} d t_{i} \Phi_{0}\left(t_{i}\right) \frac{3: \mathcal{B}_{1}\left(t_{12}\right) \mathcal{B}_{1}\left(t_{34}\right) \mathcal{B}_{2}\left(t_{56}\right):+\epsilon^{2}\left(: \mathcal{B}_{2}\left(t_{12}\right) \mathcal{B}_{2}\left(t_{34}\right) \mathcal{B}_{2}\left(t_{56}\right):+6: \mathcal{B}_{1} \mathcal{B}_{2} \mathcal{B}_{3}:+3: \mathcal{B}_{1} \mathcal{B}_{1} \mathcal{B}_{4}:\right)}{\left(2 \sin \frac{t_{12}}{2}\right)^{2 \Delta}\left(2 \sin \frac{t_{34}}{2}\right)^{2 \Delta}\left(2 \sin \frac{t_{56}}{2}\right)^{2 \Delta}} \\
& \left.\left.+\frac{1}{24} \epsilon^{4} \int \prod_{i=1}^{8} d t_{i} \Phi_{0}\left(t_{i}\right) \frac{: \mathcal{B}_{1}\left(t_{12}\right) \mathcal{B}_{1}\left(t_{34}\right) \mathcal{B}_{1}\left(t_{56}\right) \mathcal{B}_{1}\left(t_{78}\right):+\epsilon^{2}\left(6: \mathcal{B}_{1}\left(t_{12}\right) \mathcal{B}_{1}\left(t_{34}\right) \mathcal{B}_{2}\left(t_{56}\right) \mathcal{B}_{2}\left(t_{78}\right):+4: \mathcal{B}_{1} \mathcal{B}_{1} \mathcal{B}_{1} \mathcal{B}_{3}:\right)}{\left(2 \sin \frac{t_{12}}{2}\right)^{2 \Delta}\left(2 \sin \frac{t_{34}}{2}\right)^{2 \Delta}\left(2 \sin \frac{t_{56}}{2}\right)^{2 \Delta}\left(2 \sin \frac{t_{78}}{2}\right)^{2 \Delta}}+\cdots\right)\right],
\end{aligned}
$$

where $\mathcal{B}_{n}\left(t_{i j}\right) \equiv \mathcal{B}_{n}\left(t_{i}, t_{j}\right), \mathcal{D} \mu[f] \equiv \mathcal{D} f(t) / f^{\prime}(t)$ is an $\operatorname{SL}(2, R)$-invariant measure [23], $\bar{S}_{0}=-N \int d t_{1} d t_{2} \Phi_{0}\left(t_{1}\right) \Phi_{0}\left(t_{2}\right) /$ $\left(2 \sin \left(t_{12} / 2\right)\right)^{2 \Delta}$ is the same as Eq. (2.22) with $f(t)=\tan (t / 2)$ without soft mode $\epsilon k(t)$ correction, and we have dropped the odd-leg source term considering that $\left\langle\mathcal{B}_{1}\left(t_{12}\right)\right\rangle=0$ or $\langle k(t)\rangle=0 .: \cdots:$ means the time ordering. From the functional $Z$, one can reads $2 n$-point functions as

$$
\left\langle\prod_{i=1}^{2 n} \mathcal{O}_{\Phi_{0}}\left(t_{i}\right)\right\rangle=\left.\frac{1}{Z\left(\Phi_{0}\right)} \prod_{i=1}^{2 n} \frac{\delta}{\delta \Phi_{0}\left(t_{i}\right)} Z\left(\Phi_{0}\right)\right|_{\Phi_{0} \rightarrow 0}
$$

From the generating functional $W\left[\Phi_{0}\right]$, one can read all connected $2 n$-points functions from irreducible Feynman diagrams. Thus, as the leading expansion, the two-, four-, six- and eight-point functions are, respectively, given by

$$
\begin{aligned}
G_{2} & =N \frac{1+\epsilon^{2}\left\langle\mathcal{B}_{2}\left(t_{12}\right)\right\rangle}{\left(2 \sin \frac{t_{12}}{2}\right)^{2 \Delta}}, \quad G_{4}=N \frac{\epsilon^{2}\left\langle: \mathcal{B}_{1}\left(t_{12}\right) \mathcal{B}_{1}\left(t_{34}\right):\right\rangle}{\left(2 \sin \frac{t_{12}}{2}\right)^{2 \Delta}\left(2 \sin \frac{t_{34}}{2}\right)^{2 \Delta}}, \\
G_{6} & =N \frac{\epsilon^{4}\left\langle: \mathcal{B}_{1}\left(t_{12}\right) \mathcal{B}_{2}\left(t_{34}\right) \mathcal{B}_{1}\left(t_{56}\right):\right\rangle}{\left(2 \sin \frac{t_{12}}{2}\right)^{2 \Delta}\left(2 \sin \frac{t_{34}}{2}\right)^{2 \Delta}\left(2 \sin \frac{t_{6}}{2}\right)^{2 \Delta}}, \quad G_{8}=N \frac{\epsilon^{4}\left\langle: \mathcal{B}_{1}\left(t_{12}\right) \mathcal{B}_{1}\left(t_{34}\right) \mathcal{B}_{1}\left(t_{56}\right) \mathcal{B}_{1}\left(t_{78}\right):\right\rangle}{\left(2 \sin \frac{t_{12}}{2}\right)^{2 \Delta}\left(2 \sin \frac{t_{34}}{2}\right)^{2 \Delta}\left(2 \sin \frac{t_{6}}{2}\right)^{2 \Delta}\left(2 \sin \frac{t_{78}}{2}\right)^{2 \Delta}} . \\
G_{10} & =N \frac{\epsilon^{6}\left\langle: \mathcal{B}_{1}\left(t_{12}\right) \mathcal{B}_{1}\left(t_{34}\right) \mathcal{B}_{2}\left(t_{56}\right) \mathcal{B}_{1}\left(t_{78}\right) \mathcal{B}_{1}\left(t_{9,10}\right):\right\rangle}{\left(2 \sin \frac{t_{12} 2}{2}\right)^{2 \Delta}\left(2 \sin \frac{t_{34}}{2}\right)^{2 \Delta}\left(2 \sin \frac{t_{56}}{2}\right)^{2 \Delta}\left(2 \sin \frac{t_{78}}{2}\right)^{2 \Delta}\left(2 \sin \frac{t_{9,10}}{2}\right)^{2 \Delta}},
\end{aligned}
$$

where $G_{2}=G_{2}\left(t_{1}, t_{2}\right), G_{4}=G_{4}\left(t_{1}, t_{2}, t_{3}, t_{4}\right)$, etc. : $\cdots:$ means the time ordering and $\langle\cdots\rangle \equiv Z_{0}^{-1} \int \mathcal{D} \mu[f] \cdots e^{-\bar{S}_{0}}$ and $Z_{0}=\left.Z\left(\Phi_{0}\right)\right|_{\Phi_{0} \rightarrow 0}$. The generalization to more higher-order $2 n$-point functions is straightforward. 


\section{E. Loop corrections to two-point function}

\section{Zero temperature case}

By using the correlation function $\mathcal{B}_{2}\left(t_{1}, t_{2}\right)$ in Eq. (2.36) and the soft mode propagators in Eq. (2.11), one obtains the correction to the two-point function as

$\epsilon^{2}\left\langle\mathcal{B}_{2}\left(t_{12}\right)\right\rangle=\frac{1}{18 \pi C}\left[\Delta\left(\pi t_{12}-\frac{t_{12}^{2}}{4}\right)-\pi \Delta^{2} t_{12}\right]$.

In the large Lorentzian time with $t \rightarrow i \hat{t}$ and in the contour chosen in Eq. (4.24), the loop correction to the two-point function turns out to be Lorentzian time independent as

$$
\epsilon^{2}\left\langle\mathcal{B}_{2}\left(t_{12}\right)\right\rangle=\frac{\pi(3-4 \Delta) \Delta}{72 C} .
$$

\section{Finite temperature case}

By using the correlation function $\mathcal{B}_{2}\left(t_{1}, t_{2}\right)$ in Eq. (2.42) and the soft mode propagators in Eq. (2.17), one obtains the loop corrections to the two-point function as

$$
\begin{aligned}
\epsilon^{2}\left\langle\mathcal{B}_{2}\left(t_{12}\right)\right\rangle= & \frac{1}{2 \pi C}\left[\frac { \Delta } { 4 \operatorname { s i n } ^ { 2 } \frac { t _ { 1 1 } } { 2 } } \left(t_{12}^{2}-2 \pi t_{12}\right.\right. \\
& \left.\left.+2\left(\pi-t_{12}\right) \sin t_{12}+4 \sin ^{2} \frac{t_{12}}{2}\right)\right] \\
& \left.+\frac{\Delta^{2}}{2}\left(\frac{t_{12}-2 \pi}{\tan \frac{t_{12}}{2}}-2\right)\left(\frac{t_{12}}{\tan \frac{t_{12}}{2}}-2\right)\right],
\end{aligned}
$$

which recovers Eq. (4.36) in Ref. [5]. The Feynman diagrams of the loop corrections from pNGBs are depicted in Fig. 1. In the large Lorentzian time with $t \rightarrow i \hat{t}$ and in the contour chosen in Eq. (4.24), one has the two-point functions as

$$
\epsilon^{2}\left\langle\mathcal{B}_{2}\left(t_{12}\right)\right\rangle=\frac{1}{2 \pi C}\left[\left(1-\frac{\pi^{2}}{4}\right) \Delta+2 \Delta^{2}\right] \sim \frac{\pi}{\beta} \Delta^{2},
$$

which is a constant and independent of $\hat{t}$.

\section{F. Three-point correlation functions}

The three-point function of the dual single-trace operator can be expanded as

$G\left(t_{1}, t_{2}, t_{3}\right)=G_{0}\left(t_{1}, t_{2}, t_{3}\right)\left[1+\epsilon \mathcal{C}_{1}\left(t_{123}\right)+\epsilon^{2} \mathcal{C}_{2}\left(t_{123}\right)+\epsilon^{2}\right]$,

where $\mathcal{C}_{n}\left(t_{i j k}\right) \equiv \mathcal{C}_{n}\left(t_{i j}, t_{j k}, t_{k i}\right)$, with $t_{i j}=\left|t_{i}-t_{j}\right|$. By neglecting the perturbation expansion term, $\epsilon \rightarrow 0$, the $\mathrm{AdS}_{2}$ thermal two-point functions is recovered as

$$
\begin{aligned}
G_{0}\left(t_{1}, t_{2}, t_{3}\right) & =\frac{C_{3}}{\left[2 \sin \left(\frac{t_{12}}{2}\right)\right]^{\Delta}\left[2 \sin \left(\frac{t_{23}}{2}\right)\right]^{\Delta}\left[2 \sin \left(\frac{t_{13}}{2}\right)\right]^{\Delta}} \stackrel{T=0}{=} \frac{C_{3}}{t_{12}^{\Delta} t_{23}^{\Delta} t_{13}^{\Delta}} \\
& \rightarrow \frac{C_{3}(\pi / \beta)^{3 \Delta}}{\left(\sin \frac{\pi t_{12}}{\beta}\right)^{\Delta}\left(\sin \frac{\pi t_{23}}{\beta}\right)^{\Delta}\left(\sin \frac{\pi t_{13}}{\beta}\right)^{\Delta}}
\end{aligned}
$$

where in the last equality, we have recovered the thermal factor $t_{12} \rightarrow(2 \pi) / \beta t_{12}$. The leading correction to the thermal two-point-Green's function can be expressed more explicitly as

$$
\begin{aligned}
\mathcal{C}_{1}\left(t_{123}\right)= & \Delta\left(k^{\prime}\left(t_{1}\right)+k^{\prime}\left(t_{2}\right)+k^{\prime}\left(t_{3}\right)-\frac{k\left(t_{1}\right)-k\left(t_{2}\right)}{2 \tan \frac{t_{12}}{2}}-\frac{k\left(t_{1}\right)-k\left(t_{3}\right)}{2 \tan \frac{t_{13}}{2}}-\frac{k\left(t_{2}\right)-k\left(t_{3}\right)}{2 \tan \frac{t_{23}}{2}}\right), \\
\mathcal{C}_{2}\left(t_{123}\right)= & \frac{\Delta^{2}}{2}\left(\frac{\sin \left(t_{12}\right)+\sin \left(t_{23}\right)+\sin \left(t_{31}\right)}{4\left(\sin \frac{t_{12}}{2}\right)\left(\sin \frac{t_{23}}{2}\right)\left(\sin \frac{t_{31}}{2}\right)}\left[k^{\prime}\left(t_{1}\right)+k^{\prime}\left(t_{2}\right)+k^{\prime}\left(t_{3}\right)\right]+\frac{\left[k\left(t_{1}\right)-k\left(t_{2}\right)\right]\left(\sin \frac{t_{12}}{2}\right)^{2}}{2\left(\sin \frac{t_{12}}{2}\right)\left(\sin \frac{t_{23}}{2}\left(\sin \frac{t_{31}}{2}\right)\right.}+\left(\leftrightarrow t_{23}\right)+\left(\leftrightarrow t_{31}\right)\right)^{2} \\
& +\frac{\Delta}{4}\left(\frac{\left[k\left(t_{1}\right)-k\left(t_{2}\right)\right]^{2}}{2\left(\sin \frac{t_{12}}{2}\right)^{2}}+\frac{\left[k\left(t_{1}\right)-k\left(t_{3}\right)\right]^{2}}{2\left(\sin \frac{t_{31}}{2}\right)^{2}}+\frac{\left[k\left(t_{2}\right)-k\left(t_{3}\right)\right]^{2}}{2\left(\sin \frac{t_{23}}{2}\right)^{2}}-2\left[k^{\prime}\left(t_{1}\right)^{2}+k^{\prime}\left(t_{2}\right)^{2}+k^{\prime}\left(t_{3}\right)^{2}\right]\right)
\end{aligned}
$$

where for the briefness, we did not recover the thermal factor $\beta / 2 \pi$ for this expression. These three-point corrections can also contributes as the loop correction to the twopoint, by taking $t_{3} \rightarrow t_{2}$, Eq. (2.51) exactly reduces to be the same formalism as Eq. (2.42).

\section{Correction to three-point correlation}

By using the correlation function $\mathcal{C}_{2}\left(t_{1}, t_{2}, t_{3}\right)$ in Eq. (2.51) and the soft mode propagators in Eq. (2.17), assuming $t_{1}>t_{2}>t_{3}$, then one obtains the loop corrections to the three-point function as depicted in the first Feynman diagram in Fig. 2(a)

$$
G_{3}\left(t_{123}\right) \propto \lambda_{3} \epsilon^{2}\left\langle\mathcal{C}_{2}\left(t_{123}\right)\right\rangle,
$$

and an explicit form of the correction term turns out to be

$$
\begin{aligned}
G_{\mathcal{V}_{1} \mathcal{V}_{2} \mathcal{V}_{3}=} & \frac{\lambda_{3} C_{3}}{2 \pi C}\left[\frac { \Delta } { 2 } \left(\frac{\left(\pi-t_{12}\right)}{2 \tan \left(\frac{t_{12}}{2}\right)}+\frac{1}{2}+t_{12} \frac{\left(t_{12}-2 \pi\right)}{4 \sin ^{2}\left(\frac{t_{12}}{2}\right)}\right.\right. \\
& \left.\left.+\left(t_{12} \leftrightarrow t_{23}\right)+\left(t_{12} \leftrightarrow t_{31}\right)\right)+\mathcal{O}\left(\frac{\Delta^{2}}{2}\right)\right],
\end{aligned}
$$



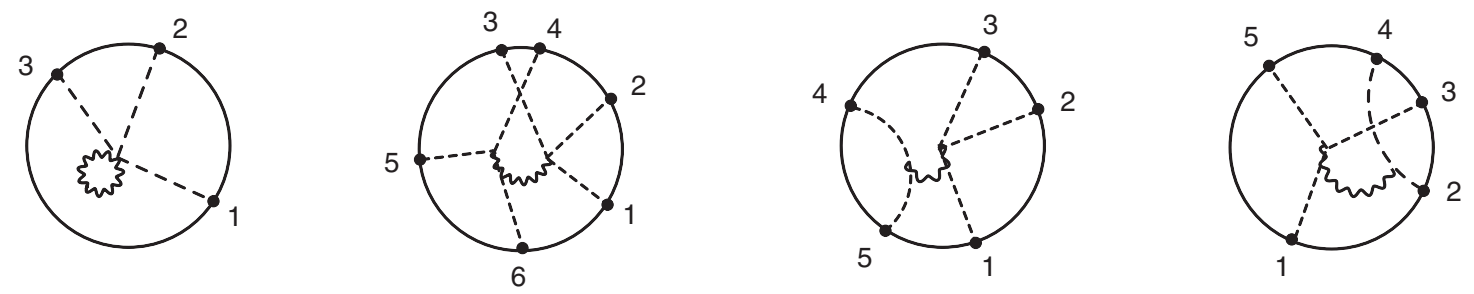

(a) $G_{3}\left(t_{1}, t_{2}, t_{3}\right)$

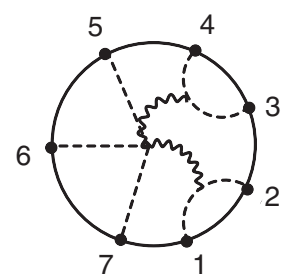

(c)

$G_{7}\left(t_{1}, t_{2}, t_{3}, t_{4}, t_{5}, t_{6}, t_{7}\right)$

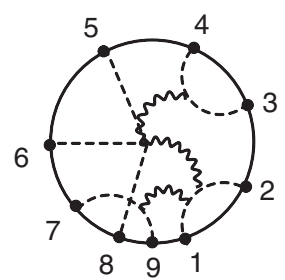

(d)

$G_{9}\left(t_{1}, t_{2}, t_{3}, t_{4}, t_{5}, t_{6}, t_{7}, t_{8}, t_{9}\right)$

FIG. 2. Typical Feynman diagrams for odd-point matter correlation functions of matter fields (dashed line) with loop corrections from soft modes (wave lines) as shown in Eq. (2.17): (a) Three-point correlation functions $G_{3}\left(t_{1}, t_{2}, t_{3}\right)$ with the corrections from gravitational soft mode to $\mathcal{C}_{1}\left(t_{123}\right)$ and $\mathcal{C}_{2}\left(t_{123}\right)$ as shown in Eq. (2.51); a six-point function $G_{6}\left(t_{123456}\right) \propto \lambda_{3}^{2} \epsilon^{2}\left\langle\mathcal{C}_{1}\left(t_{123}\right) \mathcal{C}_{1}\left(t_{456}\right)\right\rangle$ diagram is also shown on the right; (b) Five-point correlation functions $G_{5}\left(t_{12345}\right) \propto \lambda_{3} \epsilon^{2}\left\langle\mathcal{C}_{1}\left(t_{123}\right) \mathcal{B}_{1}\left(t_{45}\right)\right\rangle$; (c) Seven-point correlation functions $G_{7}\left(t_{1234567}\right) \propto \lambda_{3} \epsilon^{4}\left\langle\mathcal{C}_{2}\left(t_{123}\right) \mathcal{B}_{1}\left(t_{45}\right) \mathcal{B}_{1}\left(t_{67}\right)\right\rangle ;(\mathrm{d})$ Ninepoint correlation functions $G_{9}\left(t_{123456789}\right) \propto \lambda_{3} \epsilon^{5}\left\langle\mathcal{C}_{2}\left(t_{123}\right) \mathcal{B}_{1}\left(t_{45}\right) \mathcal{B}_{1}\left(t_{67}\right) \mathcal{B}_{1}\left(t_{89}\right)\right\rangle$.

where the coefficient $C$ comes from that associated with the soft mode propagators. Since the terms at order $\Delta^{2}$ is length, we will not list here.

\section{Correction to five-point correlation}

The correction to the five-point correction turns out to be

$$
G_{5}\left(t_{12345}\right) \propto \lambda_{3} \epsilon^{3}\left\langle\mathcal{C}_{2}\left(t_{123}\right) \mathcal{B}_{1}\left(t_{45}\right)\right\rangle .
$$

For the TOC's of five-point correlation function with normal time order $t_{1}>t_{2}>t_{3}>t_{4}>t_{5}$, one obtains the loop corrections to the correlation function as depicted in the first Feynman diagram in Fig. 2(b),

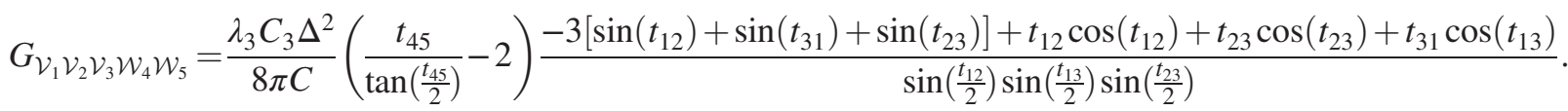

\section{QUANTUM LIQUID WITH SCHWARZIAN CORRECTIONS}

In this section, we study the retarded Green's functions as well as the local spin-spin correlation functions of the quantum liquid with Schwarzian correction in terms of the "Schwarzian liquid," which can be related to the spectral functions and local dynamical susceptibility of strongly interacting quantum liquid including the spin liquid phase [2,38], i.e., MFL [42] or FFL [43]. We also generalize the $\mathrm{AdS}_{2}$ vacuum in two-dimensional gravity to a higher-dimensional $\mathrm{RN}-\mathrm{AdS}_{d+1}$ vacuum in Einstein gravity with the Maxwell action as in Appendix C. We obtain the exact formula of $T=0$ residual entropy of a generic $\mathrm{SYK}_{p}$ model as in Appendix C 4. Moreover, the analytic formula of a new gauge covariant optical conductivity for $(0+1)$-dimensional quantum critical system is obtained in Appendix C 5.

\section{A. Quantum liquid from $\mathbf{A d S}_{2}$}

\section{Poincaré $\mathrm{AdS}_{2}$ :zero temperature $\mathrm{CFT}_{1}$}

In the $\mathrm{AdS}_{2}$ spacetime in the energy coordinate $z$, the metric and the gauge field is linear in $1+1$-dimensional spacetime as

$$
d s^{2}=-\frac{1}{4 c_{\mu}} \frac{\ell^{2}}{z^{2}}\left(-d t^{2}+d z^{2}\right), \quad A_{t}=\mu\left(1-\frac{z_{\star}}{z}\right),
$$

which is a $\mathrm{AdS}_{2}$ spacetime in the Poincare coordinate, $c_{\mu}$ contains UV information from a two-dimensional gravity. 
For the convenience, one may define an effective $\mathrm{AdS}_{2}$ radius as

$$
\ell_{L}^{2} \equiv-\frac{1}{4 c_{\mu}} \ell^{2}
$$

In the momentum spacetime, the Klein-Gordon equation of a charge scalar in out-wave $e^{-i \omega t+i k x}\left(\partial_{t} \rightarrow-i \omega, \partial_{x} \rightarrow i k\right)$ becomes

$\left[\partial_{z}^{2}+\left(\omega+q \mu\left(1-\frac{z_{\star}}{z}\right)\right)^{2}-\frac{m^{2} \ell^{2}}{z^{2}}\right] \phi(z, k)=0$,

which leads to the wave functions

$\phi(z)=c_{1} M_{i q \mu z_{\star},-\nu_{q}}(2 i z \bar{\omega})+c_{2} W_{i q \mu z_{\star},-\nu_{q}}(2 i z \bar{\omega})$,

where $\bar{\omega}=\omega+q \mu$ and the conformal dimension is

$$
\nu_{q}=\sqrt{\frac{1}{4}-\frac{m^{2} \ell^{2}}{4 c_{\mu}}-q^{2} \mu^{2} z_{\star}^{2}} .
$$

In the special case with $c_{\mu}=-1 / 4$, it just recovers the original one.

In the near horizon limit, one obtains the asymptotic behavior of the boson wave function as

$$
\begin{aligned}
& \phi(z)^{z \rightarrow \infty} 2^{i q \mu z_{\star}}\left(\frac{c_{1}(-1)^{\frac{1}{2}-\nu_{q}+i q \mu z_{\star}} \Gamma\left(1-2 \nu_{q}\right)}{\Gamma\left(i q \mu z_{\star}-\nu_{q}+\frac{1}{2}\right)}+c_{2}\right) \\
& \quad \times(i \bar{\omega})^{i q \mu z_{\star}} e^{-i \bar{\omega} z} z^{i q \mu z_{\star}}+\frac{c_{1} \Gamma\left(1-2 \nu_{q}\right) 2^{-i q \mu z_{\star}}(i \bar{\omega})^{-i q \mu z_{\star}}}{\Gamma\left(-i q \mu z_{\star}-\nu_{q}+\frac{1}{2}\right)} \\
& \quad \times e^{i \bar{\omega} z} z^{-i q \mu z_{\star}} .
\end{aligned}
$$

The out-going wave is $e^{-i \omega t+i \bar{\omega} z-i q \mu z_{\star} \ln z}$, which implies that the in-falling boundary condition to be $c_{1}=0$. On the other hand, in the infinite boundary $z \rightarrow 0$, one has $\phi(z) \stackrel{z \rightarrow 0}{\sim} B(\omega) z^{\nu_{q}+\frac{1}{2}}+A(\omega) z^{-\nu_{q}+\frac{1}{2}}$, where $A$ and $B$ are identified as source and response, respectively, and can be expressed more explicitly as

$$
\begin{aligned}
& A(\omega)=\frac{2^{\frac{1}{2}-\nu_{q}}(i \bar{\omega})^{\frac{1}{2}-\nu_{q}}\left[c_{2} \Gamma\left(2 \nu_{q}\right)+c_{1} \Gamma\left(-i q \mu z_{\star}+\nu_{q}+\frac{1}{2}\right)\right]}{\Gamma\left(-i q \mu z_{\star}+\nu_{q}+\frac{1}{2}\right)}, \\
& B(\omega)=\frac{c_{2} 2^{\nu_{q}+\frac{1}{2}}(i \bar{\omega})^{\nu_{q}+\frac{1}{2}} \Gamma\left(-2 \nu_{q}\right)}{\Gamma\left(-i q \mu z_{\star}-\nu_{q}+\frac{1}{2}\right)} .
\end{aligned}
$$

The two-point Green's function ban be read as

$$
G(\omega)=4^{-\nu_{q}}(i \bar{\omega})^{-2 \nu_{q}} \frac{\Gamma\left(2 \nu_{q}\right)}{\Gamma\left(-2 \nu_{q}\right)} \frac{\Gamma\left(-i q \mu z_{\star}-\nu_{q}+\frac{1}{2}\right)}{\Gamma\left(-i q \mu z_{\star}+\nu_{q}+\frac{1}{2}\right)} .
$$

By doing an inverse Fourier transformation, one has

$$
\begin{aligned}
\int_{-\infty}^{+\infty} d \omega e^{-i \omega t} \omega^{a}= & -e^{\frac{i \pi a}{2} \sin (\pi a) \Gamma(a+1)} \\
& \times \begin{cases}\frac{\operatorname{sgn}^{-1}(t)+1}{|t|^{a+1}}, & a>0 \\
\frac{\operatorname{sgn}(t)+1}{|t|^{a+1}}, & a<0 .\end{cases}
\end{aligned}
$$

$\int_{-\infty}^{+\infty} d \omega e^{-i \omega t}|\omega|^{a}=-2 \sin \left(\frac{\pi a}{2}\right) \frac{\Gamma(a+1)}{|t|^{a+1}}$,

where $e^{-i \pi a}=(-1)^{a}=(-i)^{2 a}$ and $\operatorname{sgn}(\mathrm{t})=t /|t|$. Thus, in the coordinate spacetime, assuming $t \gg 0$, then one obtains the retarded Green's function in real coordinate spacetime, which just recovers the ansatz of the form of the two-point correlation function at strong coupling at zero temperature $[5,29]$,

$G(t)=\sqrt{\frac{2}{\pi}} e^{-i \pi \nu_{q}} \sin \left(2 \pi \nu_{q}\right) \Gamma\left(1-2 \nu_{q}\right) \frac{\operatorname{sgn}(t)}{|t|^{1-2 \nu_{q}}} \sim b \frac{\operatorname{sgn}(t)}{|t|^{2 \Delta_{+}}}$.

It is worthy of noticing that the result reproduces the SYK uniform saddle-point solution, by making a match as below

$$
\begin{aligned}
b & \equiv\left(\frac{1}{2 \pi J^{2}}\left(1-\frac{2}{p}\right) \tan \frac{\pi}{p}\right)^{1 / p} \\
& =\sqrt{\frac{2}{\pi}} e^{-i \pi \nu_{q}} \sin \left(2 \pi \nu_{q}\right) \Gamma\left(1-2 \nu_{q}\right),
\end{aligned}
$$

where $\Delta \equiv p^{-1}=1 / 2-\nu_{q}$. In particularly, in the case that $\nu_{q}=1 / 4, m^{2} \ell^{2} /\left(4 c_{\mu}\right)=q^{2} \mu^{2} z_{\star}^{2}$, the Green's function just recovers the two-point function of SYK model with conformal dimension $\Delta_{ \pm}^{\mathrm{IR}}=1 / 4$ due to an emergent conformal symmetry at low energies and large $N$ at zero temperature,

$$
G(t)=\left\langle\left[\mathcal{O}_{\Delta}(t), \mathcal{O}_{\Delta}(0)\right]\right\rangle \sim \frac{\operatorname{sgn}(t)}{\sqrt{|t|}}, \quad t \gg 1,
$$

where $\sim$ denotes a common factor $\sqrt{2} e^{-\frac{i \pi}{4}}$ is dropped. It is useful to use the Fourier transforms for symmetric and antisymmetric function as $\int_{-\infty}^{+\infty} e^{i \omega t}|t|^{-2 \Delta}\{\operatorname{sgn}(t), 1\}=$ $2 \Gamma(1-2 \Delta)|\omega|^{2 \Delta-1}\{i \cos (\pi \Delta) \operatorname{sgn}(\omega), \sin (\pi \Delta)\}$.

\section{Global $\mathrm{AdS}_{2}$ :finite temperature $\mathrm{CFT}_{1}$}

Consider the $\mathrm{AdS}_{2}$ metric in global coordinates as in (B5) for hyperbolic case in the vacuum $b=1$ as

$d s^{2}=-\frac{1}{4 c_{\mu}} \frac{-d t^{2}+d z^{2}}{z_{0}^{2} \sinh ^{2}\left(z / z_{0}\right)}, \quad A_{t}(z)=\mu z_{0}\left(1-\operatorname{coth} \frac{z}{z_{0}}\right)$, 
where $z \in(0, \infty)$. It is worthy of noticing that it can be transformed into

$d s^{2}=-\frac{1}{4 c_{\mu}} \frac{-d t^{2}+d z^{2}}{\sinh ^{2} z}, \quad A_{t}(z)=\mu(1-\operatorname{coth} z)$,

by making a replacement

$$
t \rightarrow t z_{0}, \quad z \rightarrow z z_{0}, \quad \mu \rightarrow \frac{\mu}{z_{0}} .
$$

For the simplicity, let's consider the $z_{0}=1$ case at the beginning, we can obtain a general results by making an inverse rescaling

$$
\omega \rightarrow \omega z_{0}, \quad z \rightarrow \frac{z}{z_{0}}, \quad \mu \rightarrow \mu z_{0}, \quad z_{0}=\frac{1}{2 \pi T}
$$

the parameter $z_{0}$ is related to the temperature, according to Eq. (B3). The Klein-Gordan equation is

$$
\begin{gathered}
\phi^{\prime \prime}(z)+\left[\omega^{2}+\left(\frac{1}{4}-\nu_{1}^{2}\right) \frac{1}{\sinh z}\right] \phi(z)=0, \\
\nu_{1}=\sqrt{\frac{1}{4}-\frac{m^{2}}{4 c_{\mu}}} .
\end{gathered}
$$

For neutral scalar case $(\mu=0)$, the wave functions are

$$
\begin{aligned}
\phi(z)= & i^{-2 \nu_{1}} \tanh ^{\frac{1}{2}-\nu_{1}}(z)\left(-\operatorname{sech}^{2}(z)\right)^{-\frac{i \omega}{2}}\left[c_{2} \tanh ^{-\nu_{1}}(z)_{2} F_{1}\left(\frac{1}{4}\left(-2 \nu_{1}-2 i \omega+1\right), \frac{1}{4}\left(-2 \nu_{1}-2 i \omega+3\right) ; 1-\nu_{1} ; \tanh ^{2}(z)\right)\right. \\
& \left.+c_{1} i^{2 \nu_{1}} \tanh ^{\nu_{1}}(z)_{2} F_{1}\left(\frac{1}{4}\left(2 \nu_{1}-2 i \omega+1\right), \frac{1}{4}\left(2 \nu_{1}-2 i \omega+3\right) ; \nu_{1}+1 ; \tanh ^{2}(z)\right)\right] .
\end{aligned}
$$

For the charged scalar case, the Klein-Gordan equation is

$$
\phi^{\prime \prime}(z)+\left[\omega^{2}+\left(\frac{1}{4}-\nu_{1}^{2}\right) \frac{1}{\sinh ^{2} z}+2 q \mu \bar{\omega}(1-\operatorname{coth} z)\right] \phi(z)=0, \quad \nu_{1}=\sqrt{\frac{1}{4}-\frac{m^{2}}{4 c_{\mu}}-q^{2} \mu^{2}} .
$$

The wave functions are

$$
\begin{aligned}
\phi(z)= & -i \cosh (z) \tanh ^{\frac{1}{2}-\nu_{1}}(z)(\tanh (z)+1)^{-\frac{1}{2} i(2 \mu q+\omega+i)}(\tanh (z)-1)^{\nu_{1}+i \mu q+\frac{i \omega}{2}} \\
& \times\left[c_{1}\left(\frac{\tanh (z)}{\tanh (z)-1}\right)^{-\nu_{1}}{ }_{2} F_{1}\left(-i q \mu-\nu_{1}+\frac{1}{2},-i q \mu-\nu_{1}-i \omega+\frac{1}{2} ; 1-2 \nu_{1} ; \frac{2 \tanh (z)}{\tanh (z)-1}\right)\right. \\
& +c_{2}(-2)^{2 \nu_{1}}\left(\frac{\tanh (z)}{\tanh (z)-1}\right)^{\nu_{1}}{ }_{2} F_{1}\left(-i q \mu+\nu_{1}+\frac{1}{2},-i q \mu+\nu_{1}-i \omega+\frac{1}{2} ; 1+2 \nu_{1} ; \frac{2 \tanh (z)}{\tanh (z)-1)] .}\right.
\end{aligned}
$$

The wave function can also be reexpressed in a new coordinate $\rho \equiv \tanh z$ as

$$
\begin{aligned}
\phi(\rho)= & -\frac{i \rho^{\frac{1}{2}-\nu_{1}}(\rho+1)^{-\frac{1}{2} i(2 \mu q+\omega+i)}(\rho-1)^{\nu_{1}+i \mu q+\frac{i \omega}{2}}}{\sqrt{1-\rho^{2}}}\left[c_{12} F_{1}\left(-i q \mu-\nu_{1}+\frac{1}{2},-i q \mu-\nu_{1}-i \omega+\frac{1}{2} ; 1-2 \nu_{1} ; \frac{2 \rho}{\rho-1}\right)\right. \\
& \left.+c_{2}(-2)^{2 \nu_{1}}\left(\frac{\rho}{\rho-1}\right)^{2 \nu_{1}}{ }_{2} F_{1}\left(-i q \mu+\nu_{1}+\frac{1}{2},-i q \mu+\nu_{1}-i \omega+\frac{1}{2} ; 1+2 \nu_{1} ; \frac{2 \rho}{\rho-1}\right)\right] .
\end{aligned}
$$

In the near horizon limit $(\rho \rightarrow 1)$, the wave function can be reexpressed as $\psi(\rho)^{\rho \rightarrow 1} \sim a(\omega)(1-\rho)^{\frac{i \omega}{2}}+b(\omega)(1-\rho)^{-\frac{i \omega}{2}}$, where $\sim$ means that we have dropped a common factor $-i 2^{\nu_{1}-\frac{1}{2}}(-1)^{\nu_{1}+i \mu q+\frac{i \omega}{2}}$ in front of the wave function and the coefficients are

$$
\begin{aligned}
& a(\omega)=2^{-\frac{i \omega}{2}} \Gamma(-i \omega)\left(\frac{c_{1} \Gamma\left(1-2 \nu_{1}\right)}{\Gamma\left(i q \mu-\nu_{1}+\frac{1}{2}\right) \Gamma\left(-i q \mu-\nu_{1}-i \omega+\frac{1}{2}\right)}+\frac{c_{2} \Gamma\left(2 \nu_{1}+1\right)}{\Gamma\left(i q \mu+\nu_{1}+\frac{1}{2}\right) \Gamma\left(-i q \mu+\nu_{1}-i \omega+\frac{1}{2}\right)}\right), \\
& b(\omega)=2^{\frac{i \omega}{2}} \Gamma(i \omega)\left(\frac{c_{1} \Gamma\left(1-2 \nu_{1}\right)}{\Gamma\left(-i q \mu-\nu_{1}+\frac{1}{2}\right) \Gamma\left(i q \mu-\nu_{1}+i \omega+\frac{1}{2}\right)}+\frac{c_{2} \Gamma\left(1+2 \nu_{1}\right)}{\Gamma\left(-i q \mu+\nu_{1}+\frac{1}{2}\right) \Gamma\left(i q \mu+\nu_{1}+i \omega+\frac{1}{2}\right)}\right) .
\end{aligned}
$$

Since $e^{-i \omega t-i \frac{1}{2} \ln (1-\rho)}$ is the infalling wave, will impose the in-falling wave condition that $a(\omega)=0$, from which the relation between $c_{2}$ and $c_{1}$ can be determined. In the UV limit, one has 


$$
\phi(z)^{z \rightarrow 0} c_{1} \rho^{\frac{1}{2}-\nu_{1}}+c_{2} 4^{\nu_{1}} e^{4 i \pi \nu_{1}} \rho^{\frac{1}{2}+\nu_{1}} \equiv A(\omega) \rho^{\Delta_{-}}+B(\omega) \rho^{\Delta_{+}}
$$

where for $\sim$, we have dropped a common factor $-i(-1)^{\nu_{1}+i \mu q+\frac{i \omega}{2}}$. The conformal dimension is defined as $\Delta_{ \pm}=1 / 2 \pm \nu_{1}$. Thus, the retarded Green's functions are

$$
G^{R}(\omega)=\frac{B(\omega)}{A(\omega)}=4^{\nu_{1}} e^{4 i \pi \nu_{1}} \frac{c_{2}}{c_{1}}=-4^{\nu_{1}} e^{4 i \pi \nu_{1}} \frac{\Gamma\left(1-2 \nu_{1}\right) \Gamma\left(i q \mu+\nu_{1}+\frac{1}{2}\right) \Gamma\left(-i q \mu+\nu_{1}-i \omega+\frac{1}{2}\right)}{\Gamma\left(1+2 \nu_{1}\right) \Gamma\left(i q \mu-\nu_{1}+\frac{1}{2}\right) \Gamma\left(-i q \mu-\nu_{1}-i \omega+\frac{1}{2}\right)} .
$$

By using the rescaling relation in Eq. (3.16), one obtains the Green's function as

$$
\begin{aligned}
G^{R}(\omega)=z_{0}^{2 \Delta_{-}} \frac{B(\omega)}{A(\omega)} & =4^{\nu_{1}} z_{0}^{2 \nu_{1}-1} e^{4 i \pi \nu_{1}} \frac{c_{2}}{c_{1}} \\
& =-2(\pi T)^{1-2 \nu_{1}} e^{4 i \pi \nu_{1}} \frac{\Gamma\left(1-2 \nu_{1}\right) \Gamma\left(i \frac{q \mu}{2 \pi T}+\nu_{1}+\frac{1}{2}\right) \Gamma\left(+\nu_{1}-i \frac{\omega+q \mu}{2 \pi T}+\frac{1}{2}\right)}{\Gamma\left(1+2 \nu_{1}\right) \Gamma\left(i \frac{q \mu}{2 \pi T}-\nu_{1}+\frac{1}{2}\right) \Gamma\left(-\nu_{1}-i \frac{\omega+q \mu}{2 \pi T}+\frac{1}{2}\right)},
\end{aligned}
$$

where the prefactors $z_{0}$ are due to the rescaling of the coordinates in Eq. (3.15). For neutral case, i.e., $\mu=0$ and $q=0$, one has

$$
G^{R}(\omega)=-4^{\nu_{1}} e^{4 i \pi \nu_{1}} \frac{\Gamma\left[2\left(1-\Delta_{+}\right)\right]}{\Gamma\left[2\left(1+\Delta_{+}\right)\right]} \frac{\Gamma\left(\Delta_{+}\right)}{\Gamma\left(1-\Delta_{+}\right)} \frac{\Gamma\left(\Delta_{+}-i \frac{\omega}{2 \pi T}\right)}{\Gamma\left(1-\Delta_{+}-i \frac{\omega}{2 \pi T}\right)},
$$

where $\Delta_{+}=1 / 2+\nu_{1}$. The equation shows that the dimension $\Delta_{+}$sets the quasinormal mode frequencies as $i \omega_{n} \beta=2 \pi\left(\Delta_{-}+n\right)$. Therefore, by doing an inverse Fourier transformation and according to the integral identity as in Eq. (D13), one obtains the retarded Green's function in real coordinate spacetime which just recovers the ansatz of the form of the two-point correlation function at strong coupling at finite temperature [5,29],

$$
G^{R}(t)=\left\langle\mathcal{O}_{\Delta}(t) \mathcal{O}_{\Delta}(0)\right\rangle=e^{2 i \pi\left(2 \Delta_{+}-1\right)} \frac{2 \Delta_{+}-1}{\Gamma\left[2\left(1+\Delta_{+}\right)\right.} \frac{\Gamma\left(\Delta_{+}\right)}{\Gamma\left(1-\Delta_{+}\right)} \frac{\pi}{\beta} \frac{\operatorname{sgn}(t)}{\left[\sinh \frac{\pi t}{\beta}\right]^{2 \Delta_{+}}} \sim b \frac{\operatorname{sgn}(t)}{\left[\frac{\beta}{\pi} \sinh \frac{\pi t}{\beta}\right]^{2 \Delta_{+}}},
$$

where $\operatorname{sgn}(t) \equiv t /|t|$ is a step function. At this step, by making a comparison with that of SYK model as in Eq. (A10), one has

$$
2 J^{2} b^{\frac{1}{\Delta_{+}}} \pi=\left(1-2 \Delta_{+}\right) \tan \pi \Delta_{+}, \quad b=e^{2 i \pi\left(2 \Delta_{+}-1\right)} \frac{2 \Delta_{+}-1}{\Gamma\left[2\left(1+\Delta_{+}\right)\right]} \frac{\Gamma\left(\Delta_{+}\right)}{\Gamma\left(1-\Delta_{+}\right)}\left(\frac{\pi}{\beta}\right)^{2 \Delta_{+}-1} .
$$

The finite temperature retarded Green's function can be expanded as

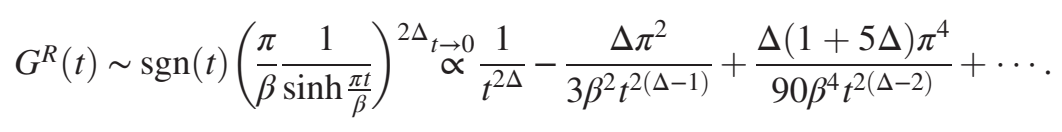

In frequency space, it can be reexpressed as $G^{R}(\omega)=$ $-i G(-i \omega+\epsilon)$. As expected, at low temperature, i.e., in the large $\beta$ limit, the retarded Green's function recovers the zero temperature one as $G^{R}(t) \sim t^{-2 \Delta}$.

For arbitrary $\Delta$, the result just recovers the retarded Greens'f function of a generic class of mean-field theories of the FFL phase of the lattice Anderson model [43], which can be obtained in an analogy procedure for fermion case, by solving the two-dimensional Dirac equation as shown in Appendix B 3.

For $\Delta=1 / 4$ case, the retarded Green's function obtained above describes a general class of strongly interacting spin liquid phase $[2,41]$ with a large density of low-energy spin excitations, or random/disordered paramagnet [38]. The spin liquid phase is due to the quantum fluctuations near a critical quantum Heisenberg spin-glass $[39,40]$, in which the quantum fluctuations are strong enough to overcome the tendency to spin-glass ordering $[2,38]$. While for $\Delta=1 / 4$, it describes a specific non-Fermi liquid (NFL) [52] in terms of the MFL phase [42].

As the SYK model in the IR limit, not only the retarded Green's function, but also the action enables exact solution, in the large $\mathrm{N}$ lmit, has the structure of a conformally invariant $(0+1)$-dimensional boundary of a $\mathrm{CFT}_{2}[38,53]$. 
The retarded Green's functions of $\mathrm{CFT}_{1}$ or $\mathrm{NCFT}_{1}$ in $\mathrm{AdS}_{2}$ and $\mathrm{NAdS}_{2}$ spacetime can be generalized to those in higher-dimensional spacetime, as explored in Appendix C.

\section{B. Schwarzian retarded Green's functions}

The two-point correlation function of FFL without Schwarzian correction in real time is

$$
G(t)=\frac{1}{i^{2 \Delta}} \frac{1}{\left[2 \frac{\beta}{2 \pi} \sinh \frac{t}{2} \frac{2 \pi}{\beta}\right]^{2 \Delta}} \stackrel{\beta \rightarrow \infty}{=} \frac{1}{i^{2 \Delta}} \frac{1}{t^{2 \Delta}},
$$

from which, one obtains the retarded Green's function, or the susceptibility of FFL as defined in Eq. (D3) as

$$
\begin{aligned}
G^{R}(\omega) & =-i \int_{-\infty}^{+\infty} d t \theta(t) e^{i \omega t} G(t) \\
& =-i\left(\frac{2 \pi}{\beta}\right)^{2 \Delta} \frac{e^{-i \pi \Delta} \Gamma(1-2 \Delta) \Gamma\left(\Delta-i \omega \frac{\beta}{2 \pi}\right)}{\Gamma\left(1-\Delta-i \omega \frac{\beta}{2 \pi}\right)},
\end{aligned}
$$

where $0<\Delta<1 / 2, \beta>0, \operatorname{Im} \omega>0$, we have used the integral in Eq. (D9) and in the last equality, we have used Euler's reflection principle. One can restore the temperature by multiplying each $\omega$ with factor $\beta /(2 \pi)$. The temperature dependent factor in front origins from thermal correlation function in Eq. (3.30), so that it recovers the quantum correlation function in the zero temperature limit. For zero temperature case, one has

$$
G^{R}(\omega)=(-1)^{-2 \Delta} \omega^{2 \Delta-1} \Gamma(1-2 \Delta),
$$

where $\Delta<1 / 2, \operatorname{Im} \omega>0$ and $(-1)^{-2 \Delta}=e^{2 i \pi \Delta}$.

The pNGBs loop corrections to the imaginary time thermal two-point functions in Eq. (2.47) can be reexpressed as real time one, by replacing $t_{12}$ with real time $i t$ as

$$
\begin{aligned}
\epsilon^{2}\left\langle\mathcal{B}_{2}\left(t_{12}\right)\right\rangle= & \frac{1}{2 \pi C}\left(\Delta\left[(2 \Delta+1)+\frac{\Delta}{2}\left(t^{2}+2 i \pi t\right)\right]\right. \\
& +\frac{\Delta(2 \Delta+1) t(t+2 i \pi)}{4 \sinh ^{2}\left(\frac{t}{2}\right)} \\
& \left.-i \Delta(2 \Delta+1)(\pi-i t) \frac{\operatorname{coth}\left(\frac{t}{2}\right)}{\sinh \left(\frac{t}{2}\right)}\right),
\end{aligned}
$$

where we will assigned every $t$ with a factor multiplying factor $2 \pi / \beta$. It can also be separated as two parts, one has even symmetry for time, while the other has odd symmetry as

$$
\begin{aligned}
\epsilon^{2}\left\langle\mathcal{B}_{2}\left(t_{12}\right)\right\rangle_{e}= & \frac{1}{2 \pi C}\left(\Delta\left[(2 \Delta+1)+\frac{\Delta}{2} t^{2}\right]\right. \\
& \left.+\frac{\Delta(2 \Delta+1) t^{2}}{4 \sinh ^{2}\left(\frac{t}{2}\right)}-\Delta(2 \Delta+1) \frac{t \cosh \left(\frac{t}{2}\right)}{\sinh \left(\frac{t}{2}\right)}\right) \\
\epsilon^{2}\left\langle\mathcal{B}_{2}\left(t_{12}\right)\right\rangle_{o}= & \frac{1}{2 \pi C}\left(\Delta^{2} t+\frac{\Delta(2 \Delta+1) t}{2 \sinh ^{2}\left(\frac{t}{2}\right)}\right. \\
& \left.-\Delta(2 \Delta+1) \frac{\cosh \left(\frac{t}{2}\right)}{\sinh \left(\frac{t}{2}\right)}\right) \pi i
\end{aligned}
$$

By doing Fourier transformation, the second and third part of the odd sector will be vanishing unless $-1<\Delta<0$, thus the nonvanishing part within $0<\Delta<1 / 2$, comes form the even part, which turns out to be

$\bar{G}^{R}(\omega)=G^{R}(\omega)\left[1+\epsilon^{2}\left\langle\mathcal{B}_{2}(\omega)\right\rangle_{e}+\epsilon^{2}\left\langle\mathcal{B}_{2}(\omega)\right\rangle_{o}\right]$,

where

$$
\begin{aligned}
\epsilon^{2}\left\langle\mathcal{B}_{2}(\omega)\right\rangle_{e}= & \frac{1}{2 \pi C}\left(\Delta(2 \Delta+1)+\frac{\Delta^{2}}{2}\left[\left(\psi_{\Delta-i \omega}^{(0)}\right)^{2}+\psi_{\Delta-i \omega}^{(1)}\right]\right. \\
& -\frac{\Delta^{2}+\omega^{2}}{2}\left[\left(\psi_{\Delta+1-i \omega}^{(0)}\right)^{2}+\psi_{\Delta+1-i \omega}^{(1)}\right] \\
& \left.-(1+2 \Delta)\left(1-i \omega \psi_{\Delta-i \omega}^{(0)}\right)\right), \\
\epsilon^{2}\left\langle\mathcal{B}_{2}(\omega)\right\rangle_{o}= & \frac{1}{2 \pi C}\left(-i \pi \Delta^{2} \psi_{\Delta-i \omega}^{(0)}\right),
\end{aligned}
$$

where we have used the integrals as in Eqs. (D13) and (D8).

\section{Zero temperature case}

For zero temperature case, one can do the Fourier transformation upon Eq. (2.45), which leads to

$$
\bar{G}^{R}(\omega)=G^{R}(\omega)\left[1+\frac{\Delta(\Delta-1)(2 \Delta-1)}{18 \pi C}\left(-\frac{i \pi}{\omega}+\frac{1}{2 \omega^{2}}\right)\right],
$$

where $\Delta<1 / 2$ and $G^{R}(t)=(-1)^{2 \Delta} \omega^{2 \Delta-1} \Gamma(1-2 \Delta)$ is given as in Eq. (3.32). The dynamical local susceptibility of Shcwarzian liquid becomes

$$
\begin{aligned}
\bar{\chi}_{\mathrm{loc}}^{(2)}(\omega) & =\int_{-\infty}^{+\infty} d t \theta(t) e^{i \omega t} \bar{G}_{2}(t) \bar{G}_{2}(-t) \\
& =\chi_{\mathrm{loc}}^{(2)}(\omega)\left(1+\frac{(2 \Delta-1)(4 \Delta-1) \Delta\left(9 C+\pi \Delta(\Delta-1)^{2}\right)}{162 \pi C^{2} \omega^{2}}+\frac{(\Delta-1)(4 \Delta-3)(2 \Delta-1)(4 \Delta-1) \Delta^{2}}{648 \pi^{2} C^{2} \omega^{4}}\right),
\end{aligned}
$$

where $\Delta<1 / 4$, and $\chi_{\mathrm{loc}}^{(2)}(\omega)=(-i \omega)^{4 \Delta-1} \Gamma(1-4 \Delta)$ as given in Eq. (3.42). After obtains loops correction from the Schwarzian effective action, the local susceptibility becomes more singular at zero frequency limit $\omega=0$. While these terms is vanishing when $\Delta=1 / 2,1 / 4$, the physical consequence of which can be observed at finite temperature. 


\section{Finite temperature case}

Therefore, for finite temperature case, the pNGBs loop corrected two-point thermal retarded Green's function becomes

$$
\begin{aligned}
\bar{G}_{T}^{R}(\omega)= & G_{T}^{R}(\omega)\left[1+\frac{1}{2 \pi C}\left(\Delta(2 \Delta+1)+\frac{\Delta^{2}}{2}\left(\left(\psi_{\Delta-i \omega \frac{\beta}{2 \pi}}^{(0)}\right)^{2}\right.\right.\right. \\
& \left.+\psi_{\Delta-i \omega \omega_{2 \pi}^{\beta}}^{(1)}\right)-\frac{\Delta^{2}+\omega^{2} \frac{\beta^{2}}{4 \pi^{2}}}{2}\left(\left(\psi_{\Delta+1-i \omega \frac{\beta}{2 \pi}}^{(0)}\right)^{2}+\psi_{\Delta+1-i \omega \frac{\beta}{2 \pi}}^{(1)}\right) \\
& \left.\left.-(1+2 \Delta)\left(1-i \omega \frac{\beta}{2 \pi} \psi_{\Delta-i \omega \frac{\beta}{2 \pi}}^{(0)}\right)-i \pi \Delta^{2} \psi_{\Delta-i \omega \frac{\beta}{2 \pi}}^{(0)}\right)\right], \\
0< & \Delta<1 / 2, \quad \beta>0, \quad \operatorname{Im} \omega>0,
\end{aligned}
$$

where $G^{R}(\omega)$ is defined in Eq. (3.31), and we have used that the iteration relation

$$
I_{\Delta+1-i \omega_{2 \pi}^{\beta}}^{(0)}=\frac{\frac{\beta^{2}}{4 \pi^{2}} \omega^{2}+\Delta^{2}}{2 \frac{\beta^{2}}{4 \pi^{2}}\left(2 \Delta^{2}+\Delta\right)} I_{\Delta-i \omega \omega_{2 \pi}^{\beta}}^{(0)},
$$

where $I_{\Delta-i \omega}^{(0)}$ is defined in Eq. (D9).

\section{High-order local spin-spin correlation}

By using the tree level retarded (real time) Green's function in Eq. (3.27), it is straightforward to calculate the local spin-spin correlation function, namely the dynamical local spin susceptibility $\chi_{\text {loc }}(\omega)$ as defined in Eq. (D5)

$$
\chi_{\mathrm{loc}}^{(2)}(\omega)=\int_{-\infty}^{+\infty} \theta(t) d t e^{i \omega t} G_{2}(t) G_{2}(-t) .
$$

According to the retarded Green's function defined in Eq. (3.30), by using Eq. (D13), we are able to calculate the local spin susceptibility at zero temperature as

$$
\begin{aligned}
\chi_{\mathrm{loc}}^{(2)}(\omega) & =\int_{-\infty}^{+\infty} d t \theta(t) \frac{e^{i \omega t}}{t^{4 \Delta}}=(-i \omega)^{-1+4 \Delta} \Gamma(1-4 \Delta) \\
& =e^{-i \frac{\pi}{2}(4 \Delta-1)} \omega^{-1+4 \Delta} \Gamma(1-4 \Delta)
\end{aligned}
$$

where the conformal dimension is limited as $0<\Delta<1 / 4$ and the frequency must be in the upper complex plane $\operatorname{Im} \omega>0$. We have also used $(-i)=e^{-i \frac{\pi}{2}}$ in the last equality of the above equation.

\section{Static and dynamic local susceptibility}

The leading-order low frequency behavior of local spin susceptibility is a constant, i.e., $\chi(\omega)=$ const. $+O(\omega)$, in which, the constant term is inverse proportional to the temperature as

$$
\chi_{\mathrm{loc}}^{(2)}(0)=\left(\frac{\beta}{\pi}\right)^{1-4 \Delta} \frac{\Gamma\left(\frac{1}{2}-2 \Delta\right) \Gamma(2 \Delta)}{2 \sqrt{\pi}} .
$$

While it turns out that for the special $\Delta=1 / 4$ case, the imaginary sector of $\chi(0)$ is divergent

$$
\chi_{\mathrm{loc}}^{(2)}(0) \propto \begin{cases}-\frac{\pi}{\beta}, & \Delta=1 / 2 \\ -\frac{1}{4 \Delta-1}+\ln \frac{2 \beta}{\pi}, & \Delta=1 / 4 .\end{cases}
$$

Consider first derivative of $\chi_{\text {loc }}^{\prime \prime}(\omega)$ with respect to frequency $\omega$, one obtains that the static local spin susceptibility $\chi_{\text {loc }}^{(3)}(0)$ is inversely proportional to the square of temperature, i.e., $\beta^{2}$ as

$\chi_{\mathrm{loc}}^{(3)}(0)=\frac{i}{2} \sqrt{\pi}\left(\frac{\beta}{\pi}\right)^{1-4 \Delta} \cot (2 \pi \Delta) \Gamma\left(\frac{1}{2}-2 \Delta\right) \Gamma(2 \Delta)$,

from which, it turns out that for the special $\Delta=1 / 2$ case, the imaginary part of $\chi_{\mathrm{loc}}^{(3)}(0)$ is divergent

$\chi_{\operatorname{loc}}^{(3)}(0) \propto \begin{cases}\frac{\pi}{\beta}\left(-\frac{1}{2 \Delta-1}+2+2 \ln \frac{\beta}{2 \pi}\right), & \Delta=1 / 2 \\ \frac{\pi^{2}}{2}, & \Delta=1 / 4 .\end{cases}$

While at the second-order derivative of $\chi_{\text {loc }}^{\prime \prime}(\omega)$ with respect to $\omega$, the static local spin susceptibility $\chi^{\prime \prime \prime \prime}(0)$ for both $\Delta=1 / 2$ and $\Delta=1 / 4$ case, becomes convergent and is inversely proportional to the cubic of temperature, i.e., $\beta^{3}$ as

$$
\chi_{\mathrm{loc}}^{(4)}(0) \propto \begin{cases}\frac{2}{3} \frac{\pi^{3}}{\beta}, & \Delta=1 / 2 \\ 14 \zeta(3), & \Delta=1 / 4\end{cases}
$$

where $\zeta(3) \approx 1.20206$ is the Riemann zeta function.

The effective bath for the local spin is given by the local spin-spin correlation function itself, which have nontrivial low frequency behavior, which appears only as a subdominant correction to the leading low frequency behavior $\chi^{(n)}(0) \sim$ const given $\beta$.

We will consider the dynamical local susceptibility in the following section.

\section{Fractionalized Fermi liquid}

For generic $\Delta$ case,

$$
G^{R}(t) \sim \frac{1}{\left[\frac{\beta}{\pi} \sinh \frac{\pi t}{\beta}\right]^{2 \Delta}} \stackrel{\beta \rightarrow \infty}{=} \frac{1}{t^{2 \Delta}},
$$

the result recovers the retarded Green's function of mean field theory of FFL [43], or generic NFL [19,35,38,54-56]. In this case, one would expect the local spin susceptibility $\chi_{\text {loc }}(\tau, \beta)$ in the large $\mathrm{N}$ limit is given by 


$$
\chi_{\mathrm{loc}}(\tau) \propto\left(\frac{\pi / \beta}{\sin \pi \tau / \beta}\right)^{4 \Delta} .
$$

For finite temperature case, by making a rescaling $t \rightarrow$ $(2 \pi / \beta) t$ or $\omega \rightarrow \beta /(2 \pi) \omega$, the dynamical local spin susceptibility at finite temperature becomes

$\chi_{\mathrm{loc}}^{(2) T}(\omega) \equiv \int_{-\infty}^{+\infty} \theta(t) d t e^{i \omega t} G_{2}\left(\frac{2 \pi}{\beta} t\right) G_{2}\left(-\frac{2 \pi}{\beta} t\right)$.

By using Eq. (3.27), one has

$$
\begin{aligned}
\chi_{\mathrm{loc}}^{(2) T}(\omega) & =\left(\frac{\beta}{2 \pi}\right)^{1-4 \Delta} \frac{\Gamma(1-4 \Delta) \Gamma\left(2 \Delta-i \frac{\beta}{2 \pi} \omega\right)}{\Gamma\left(1-2 \Delta-i \frac{\beta}{2 \pi} \omega\right)} . \\
0 & <\Delta<\frac{1}{4}, \quad \beta>0, \quad \operatorname{Im} \omega>0 .
\end{aligned}
$$

Based upon which, the higher-order local spin susceptibility with respect to the frequency becomes

$$
\begin{aligned}
& \chi_{\mathrm{loc}}^{(3) T}(\omega)=\frac{2 \pi}{\beta} \partial_{\omega} \chi_{\mathrm{loc}}^{(2) T}(\omega), \\
& \chi_{\mathrm{loc}}^{(4) T}(\omega)=\left(\frac{2 \pi}{\beta}\right)^{2} \partial_{\omega}^{2} \chi_{\mathrm{loc}}^{(2) T}(\omega) .
\end{aligned}
$$

By imposing Eq. (3.51), one obtains leading higher-order local spin susceptibility as

$$
\begin{aligned}
\left\{\chi_{\mathrm{loc}}^{(3) T}, \chi_{\mathrm{loc}}^{(4) T}, \chi_{\mathrm{loc}}^{(5) T}\right\}= & \chi_{\mathrm{loc}}^{(2) T}\left\{-\psi_{x}^{(0)},\left(\psi_{x}^{(0)}\right)^{2}+\psi_{x}^{(1)},\right. \\
& \left.-\left[\left(\psi_{x}^{(0)}\right)^{3}+3 \psi_{x}^{(0)} \psi_{x}^{(1)}+\psi_{x}^{(2)}\right]\right\},
\end{aligned}
$$

where $x \equiv 2 \Delta-i \omega \beta /(2 \pi)$, the prime is with respect to the frequency and we have used the definition of functions defined in Eq. (D10).

\section{Marginal Fermi liquid}

For $\Delta=1 / 4$ case,

$$
G^{R}(t) \sim \sqrt{\frac{\pi}{\beta \sinh \frac{\pi t}{\beta}}} .
$$

In this case, one would expect the local spin susceptibility $\chi_{\text {loc }}(\tau, \beta)$ in the large $\mathrm{N}$ limit is given by [41]

$$
\chi_{\mathrm{loc}}(\tau) \propto\left(\frac{\pi / \beta}{\sin \pi \tau / \beta}\right)+\cdots,
$$

where $\tau$ is the imaginary-time and $G(\tau)$ is the bosonic Green's function. The local susceptibility is the response to the local spin field. It is different from the uniform susceptibility $G(\tau)$, which is a consequence of the commutation relations of the spin [41]. The result also recovers the retarded Greens's function of spin liquid phase [2,41], which describes the strong quantum fluctuation in the infinite-range Heisenberg spin-glass model in the large $\mathrm{N}$ limit at low temperature.

The low frequency behavior of local spin susceptibility $\chi_{\mathrm{loc}}^{(2) T}(\omega)$ is given by Eq. (3.55).

$\chi_{\mathrm{loc}}^{(2) T}(\omega)=\ln \left(\frac{\beta}{2 \pi}\right)+\frac{1}{1-4 \Delta}-\gamma_{E}-\psi^{(0)}\left(\frac{1}{2}-i \frac{\beta \omega}{2 \pi}\right)$,

where we have used that $\psi(-1 / 2)=\psi(3 / 2)=2-\gamma_{E}-$ $2 \ln (2)$ and $\psi(1 / 2)=-\gamma_{E}-2 \ln (2)$. Thus, one obtains the universal form for low frequency behavior of the dynamical local spin-spin correlation susceptibility $[38,80]$

$$
\begin{aligned}
\chi_{\mathrm{loc}}^{\prime \prime}(\omega) & \equiv \operatorname{Im} \chi_{\mathrm{loc}}^{(2) T}(\omega)=-\operatorname{Im} \psi\left(\frac{1}{2}-i \frac{\omega \beta}{2 \pi}\right) \\
& =\frac{\pi}{2} \tanh \frac{\omega}{2 T},
\end{aligned}
$$

which is simply a smoothed-out version of the step function at zero temperature $\left(\operatorname{Im} \chi_{\operatorname{loc}}^{(2)}(\omega)=\pi \tanh (\pi \omega)\right)$ or in large frequency limit, i.e., $T \rightarrow 0$, or $T \ll \omega$. The result also recovers the local dynamical susceptibility, or spin-spin local correlations of spin liquid phase $[2,41]$. The local dynamical susceptibility implies that [52]

$$
\chi_{\mathrm{loc}}^{\prime \prime}(\omega) \propto \begin{cases}\frac{\omega}{2 T}, & \omega \ll T \\ \operatorname{sgn}(\omega), & \omega \gg T\end{cases}
$$

where the form of $\chi_{\text {loc }}^{\prime \prime}$ is precisely of the form for retarded one-particle self-energy due to exchange of spin and charge fluctuations in the phenomenological "marginal Fermi liquid" (MFL) description of High- $\mathrm{T}_{c}$ cuprates in the strange metal region [42]. In this case, at low temperature one has

$$
\chi_{\mathrm{loc}}^{\prime}(\omega) \equiv \int d \omega \frac{\chi_{\mathrm{loc}}^{\prime \prime}(\omega)}{\omega} \sim \ln \frac{1}{|\omega|}, \quad \omega \gg T .
$$

The results just recover the spin-polarization correlation function of MFL [42,52], which is a special case of NFL metal phase in a doped Mott insulator [19,35,38,55,56]. The theory of MFL assume that the self-energy $\Sigma(\omega, T)$ of the electrons behaves for $\omega>T$ like $\operatorname{Re} \Sigma(\omega, T) \sim \omega \ln |\omega|$, $\operatorname{Im} \Sigma(\omega, T) \sim|\omega|$, which is in contrast to ordinary Fermiliquid (FL) theory where $\operatorname{Re} \Sigma(\omega, T) \sim \omega, \operatorname{Im} \Sigma(\omega, T) \sim \omega^{2}$.

For higher-order local spin-spin correlation functions, at finite temperature case, one has 


$$
\begin{aligned}
& \chi_{\mathrm{loc}}^{(3) T}=\psi^{(1)}\left(\frac{1}{2}-i \frac{\beta \omega}{2 \pi}\right), \\
& \chi_{\mathrm{loc}}^{(4) T}=-\psi^{(2)}\left(\frac{1}{2}-i \frac{\beta \omega}{2 \pi}\right) .
\end{aligned}
$$

Thus, one obtains

$$
\begin{aligned}
& \operatorname{Re} \chi_{\operatorname{loc}}^{(3) T}(\omega)=\frac{\pi^{2}}{2} \operatorname{sech}^{2}\left(\frac{\beta}{2} \omega\right), \\
& \operatorname{Im} \chi_{\operatorname{loc}}^{(4) T}(\omega)=\pi^{3} \tanh \left(\frac{\beta}{2} \omega\right) \operatorname{sech}^{2}\left(\frac{\beta}{2} \omega\right),
\end{aligned}
$$

where in the last equality, we have used reflection principle in Eq. (3.61) as

$$
\begin{aligned}
\operatorname{Im} \psi\left(\frac{1}{2}+i \frac{\beta}{2 \pi} \omega\right) & =\frac{\pi}{2} \tanh \left(\frac{\beta}{2} \omega\right), \\
\operatorname{Re} \psi^{(1)}\left(\frac{1}{2}+i \frac{\beta}{2 \pi} \omega\right) & =\frac{\pi^{2}}{2} \operatorname{sech}^{2}\left(\frac{\beta}{2} \omega\right), \\
\operatorname{Im} \psi^{(2)}\left(\frac{1}{2}+i \frac{\beta}{2 \pi} \omega\right) & =\pi^{3} \tanh \left(\frac{\beta}{2} \omega\right) \operatorname{sech}^{2}\left(\frac{\beta}{2} \omega\right) .
\end{aligned}
$$

\section{Fermi liquid like}

For $\Delta=1 / 2$ case,

$$
G^{R}(t) \sim \frac{\pi}{\beta \sinh \frac{\pi t}{\beta}} .
$$

In this case, the local spin susceptibility $\chi_{\text {loc }}(\tau, \beta)$ in the large $\mathrm{N}$ limit is expected to be

$$
\chi_{\mathrm{loc}}(\tau) \propto\left(\frac{\pi / \beta}{\sin \pi \tau / \beta}\right)^{2}
$$

where $\tau$ is the imaginary-time. The low frequency behavior of local spin susceptibility $\chi_{\text {loc }}^{\prime \prime}(\omega)$ is given by

$$
\begin{aligned}
\chi_{\mathrm{loc}}^{(2) T}(\omega)= & \frac{\pi}{\beta}+i \omega\left[1-\gamma_{E}+\frac{1}{2-4 \Delta}\right. \\
& \left.+\ln \left(\frac{\beta}{2 \pi}\right)-\psi^{(0)}\left(-\frac{i \beta \omega}{2 \pi}\right)\right] .
\end{aligned}
$$

By using reflection principle in Eq. (3.61).

$$
\begin{aligned}
& \operatorname{Re} \psi\left(i \frac{\beta}{2 \pi} \omega\right) \stackrel{\omega \rightarrow 0}{\approx}-\gamma_{E}+\frac{\beta^{2} \omega^{2} \zeta(3)}{4 \pi^{2}}-\frac{\beta^{4} \omega^{4} \zeta(5)}{16 \pi^{4}}+\cdots, \\
& \operatorname{Im} \psi\left(i \frac{\beta}{2 \pi} \omega\right)=\frac{\pi}{\beta \omega}+\frac{1}{2} \pi \operatorname{coth}\left(\frac{\beta \omega}{2}\right)
\end{aligned}
$$

one obtains

$$
\begin{aligned}
\operatorname{Im} \chi_{\operatorname{loc}}^{(2) T}(\omega) & =\omega\left[1-\gamma_{E}+\frac{1}{2-4 \Delta}-\operatorname{Re} \psi^{(0)}\left(-i \frac{\beta}{2 \pi} \omega\right)\right] \\
& \stackrel{\omega \rightarrow 0}{\approx} \omega\left(1+\frac{1}{2-4 \Delta}-\frac{\beta^{2} \omega^{2} \zeta(3)}{4 \pi^{2}}\right), \\
\operatorname{Re} \chi_{\operatorname{loc}}^{(2) T}(\omega) & =\frac{\pi}{\beta}+\omega \operatorname{Im} \psi^{(0)}\left(-\frac{i \beta \omega}{2 \pi}\right)=-\frac{\omega}{2} \operatorname{coth} \frac{\beta \omega}{2} \\
& \stackrel{\omega \rightarrow 0}{\approx}-\frac{1}{\beta}-\frac{\beta \omega^{2}}{12}+\frac{\beta^{3} \omega^{4}}{720}+\mathcal{O}\left(\omega^{5}\right) .
\end{aligned}
$$

Thus,

$$
\chi_{\mathrm{loc}}^{\prime \prime}(\omega) \equiv \operatorname{Im} \chi_{\mathrm{loc}}^{(2)}(\omega) \sim \omega
$$

which indicates behavior of Fermi liquid-like. In this case,

$$
\begin{aligned}
\chi_{\mathrm{loc}}^{\prime}(\omega) & =\omega\left(2-\gamma_{E}+\frac{1}{2-4 \Delta}\right)+\frac{2 \pi}{\beta} \operatorname{Im} \ln \Gamma\left(-\frac{i \beta \omega}{2 \pi}\right) \\
& \stackrel{\omega \rightarrow 0}{\approx}\left(2+\frac{1}{2-4 \Delta}\right)+\frac{\pi^{2}}{\beta}-\frac{\beta^{2} \zeta(3)}{12 \pi^{2}} \omega^{3} .
\end{aligned}
$$

The higher-order susceptibility is

$$
\begin{aligned}
\chi_{\mathrm{loc}}^{(3)}(\omega)= & \frac{2 \pi}{\beta}\left[\frac{1}{2(1-2 \Delta)}+\ln \left(\frac{\beta}{2 \pi}\right)+1-\gamma_{E}\right. \\
& \left.-\psi^{(0)}\left(-\frac{i \beta \omega}{2 \pi}\right)\right]+i \omega \psi^{(1)}\left(-\frac{i \beta \omega}{2 \pi}\right), \\
\chi_{\mathrm{loc}}^{(4)}(\omega)= & \frac{4 \pi}{\beta} \psi^{(1)}\left(-\frac{i \beta \omega}{2 \pi}\right)-i \omega \psi^{(2)}\left(-\frac{i \beta \omega}{2 \pi}\right) .
\end{aligned}
$$

In this case, one obtains

$$
\begin{aligned}
\operatorname{Im} \chi_{\operatorname{loc}}^{(3)}(\omega) & =-\frac{2 \pi}{\beta} \operatorname{Im} \psi^{(0)}\left(-\frac{i \beta \omega}{2 \pi}\right)+\omega \operatorname{Re} \psi^{(1)}\left(-\frac{i \beta \omega}{2 \pi}\right) \\
& =\frac{\pi^{2}}{2 \beta} \frac{\sinh (\beta \omega)-\beta \omega}{\sinh ^{2} \frac{\beta \omega}{2}}, \\
\operatorname{Re} \chi_{\operatorname{loc}}^{(4)}(\omega) & =\omega \operatorname{Im} \psi^{(2)}\left(-\frac{i \beta \omega}{2 \pi}\right)+\frac{4 \pi}{\beta} \operatorname{Re} \psi^{(1)}\left(-\frac{i \beta \omega}{2 \pi}\right) \\
& =\frac{\pi^{3}}{\beta} \frac{\beta \omega \operatorname{coth}^{\left(\frac{\beta \omega}{2}\right)}-2}{\sinh ^{2} \frac{\beta \omega}{2}},
\end{aligned}
$$

where we have used the refection principle in Eq. (3.71) as

$$
\begin{aligned}
& \operatorname{Re} \psi^{(1)}\left(i \frac{\beta}{2 \pi} \omega\right)=-\frac{2 \pi^{2}}{\beta^{2} \omega^{2}}-\frac{1}{2} \pi^{2} \operatorname{csch}^{2}\left(\frac{\beta \omega}{2}\right), \\
& \operatorname{Im} \psi^{(2)}\left(i \frac{\beta}{2 \pi} \omega\right)=-\frac{8 \pi^{3}}{\beta^{3} \omega^{3}}-\pi^{3} \operatorname{coth}\left(\frac{\beta \omega}{2}\right) \operatorname{csch}^{2}\left(\frac{\beta \omega}{2}\right) .
\end{aligned}
$$




\section{Retarded Green's function}

It turns out that the loop correction from pNGBs to the thermal correlation functions, or the retarded Green's functions in Eq. (3.39), leads to a dynamically generated high-energy Hubbard band in spectral function, which corresponds to the destruction of quasiparticle states in the spectral function/DOS of quantum liquid, as shown in Fig. 3-7.

\section{1. $\Delta=1 / 4: M F L$}

In Fig. 3(a), we show the coupling strength evolution of the retarded Green's function of MFL with the Schwarzian correction in terms of "Schwarzian MFL" with $\Delta=1 / 4$ by increasing strength $\left(\sim C^{-1}\right)$ of pNGBs loop corrections to matter two-point correlation functions, due to Eq. (2.47). The $C=\infty$ case (dotted red curves) corresponds to conventional MFL with the fragile quasiparticle picture. With the increasing of the coupling strength (or decreasing of $C$ ) up to $C^{-1}=3 \pi$ (purple solid line), the DOS accumulates more in the $\omega=0$ region as the metallic phase with theFermi liquid behavior, meanwhile it develops a "slope-dig-ramp" shoulder structure, i.e., a Hubbard band at $\omega \approx 0.22$, which is dynamically generated DOS at finite frequency. The Hubbard band is a smoking gun indicating the presence of a bad metal phase. Among the intermediate range, there is a temperature dependent crossover between the Fermi liquid regime and bad metal regime in the strongly correlating regime, in which, the quasiparticle picture is still fragile or even broken down. This signature of MFL phase with
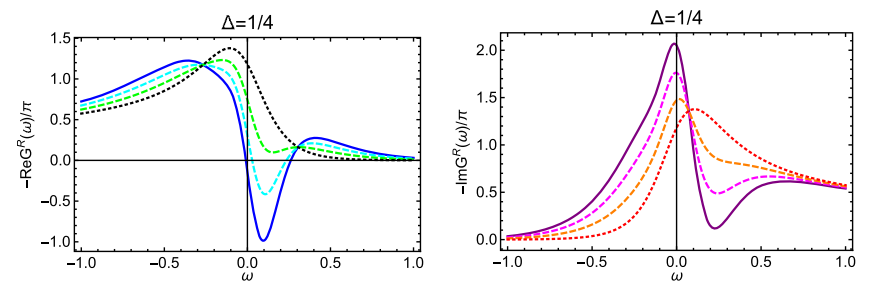

(a)Evolution of $\bar{G}^{R}(\omega)$ with coupling $(2 \pi C)^{-1}$
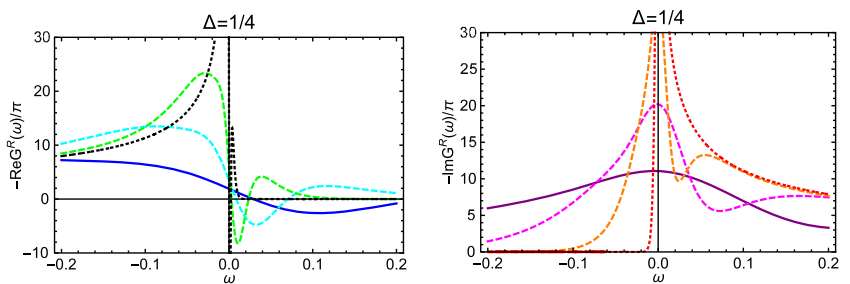

(b)Evolution of $\bar{G}^{R}(\omega)$ with temperature $\beta^{-1}$

FIG. 3. Dynamical susceptibility or retarded Green's functions of Schwarzian MFL with $\Delta=1 / 4: \bar{\chi}(\omega)=-G^{R}(\omega) / \pi$ given in Eq. (3.39): (a) Evolution with coupling strength $(2 \pi C)^{-1}: C=$ $1 / 3 \pi$ (blue/purple solid line), $C=1 / 2 \pi$ (cyan/magenta dashed line); $C=1 / \pi$ (green/orange dashed line) and $C=+\infty$ (black/ red dotted line). (b) Evolution with temperature $T=\beta^{-1}$ : In front of $\bar{\chi}(\omega)$, we have multiplying a temperature depending factor $\pi / \beta$. For different $\beta: \beta=2 \pi$ (blue/purple solid line), $\beta=20 \pi / 3$ (cyan/magenta dashed line), $\beta=20 \pi$ (green/orange dashed line); $\beta=200 \pi$ (black/red dotted line). We have chosen input parameters as $\beta=2 \pi$.
Schwarzian correction, i.e., a DOS with Hubbard band in strongly correlated region, is significant different from the conventional FL phase without such band structure. In particular, the signature of the quantum liquid can be depicted with only three input parameters: the temperature $\beta$, the conformal dimension $\Delta$ and coupling strength of lowenergy effective Schwarzian action $\sim C_{g}^{-1}$ that comprise the UV information of various two-dimensional gravity. Our analytical computation is consistent with the general tendency of the DOS calculated by DFT approach [47] or DMFT approach [49] with a state-of the art numerical calculation from first principles of many-body theory [48].

In Fig. 3(b), we also show the temperature evolution of retarded Green's functions of Schwarzian MFL with $\Delta=$ $1 / 4$ by decreasing temperature (or by increasing $\beta$ ) untill $T=1 /(200 \pi)$, which approximately corresponds to zero temperature case (dotted lines). The decreasing of the temperature from $T=1 /(2 \pi)$ to $T=1 /(20 \pi)$, the $\operatorname{dig}$ of DOS moves from $\omega \approx 0.22$ to more lower frequency region at $\omega \approx 0.02$, and so does the location of Hubbard band, which indicates that the dynamics is due to the pNGBs from spontaneous and explicit symmetry breaking. Meanwhile, the DOS accumulates rapidly and results in a peak at $\omega=0$, which implies that the quantum liquid becomes more metallic like in zero temperature limit.

In Figs. 4(a) and 4(b), we show the retarded Green's function at finite temperature with $\beta=2 \pi$ and coupling
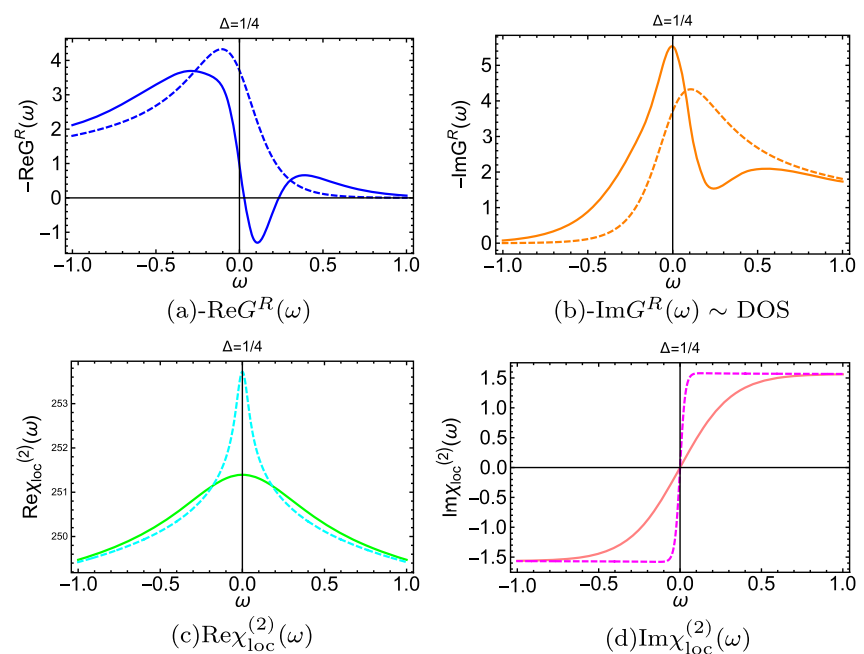

FIG. 4. Dynamical susceptibility or thermal retarded Green's functions $\chi(\omega)=G^{R}(\omega)$ given in Eq. (3.39) for quantum liquid with (solid line) or without (dashed line) Schwarzian correction. For $\Delta=1 / 4$ cases: (a) $-\operatorname{Re} G^{R}(\omega)$ (blue line); (b) $-\operatorname{Im} G^{R}(\omega)$ (orange line). We have chosen $\beta=2 \pi$ and $C=1 /(2 \pi)$. Local dynamical spin-spin correlation functions of quantum liquid $\chi_{\text {loc }}^{(2)}(\omega)$ as given in Eq. (3.51) in high temperature case with $\beta=$ $2 \pi$ (solid green/pink line) and low temperature case with $\beta=20 \pi$ (dashed cyan/magenta line): (c) $\operatorname{Re} \chi_{\text {loc }}^{(2)}(\omega),(d) \operatorname{Im} \chi_{\text {loc }}^{(2)}(\omega) \sim$ $\tanh (\omega \beta / 2)$. To avoid singularity of $\chi_{\text {loc }}^{(2)}(\omega)$ at $\Delta=1 / 4$, we have chosen $\Delta=1 / 4-\epsilon$ with $\epsilon=10^{-3}$. 
$C=1 /(2 \pi)$ for $\Delta=1 / 4$ case. It is worthy of noticing that the real part of $\chi^{(2)}(\omega)$ owns a peak at $\omega=0$ and decays with the increasing of $|\omega|$, and is expected to be a delta function $\delta(\omega)$ at $\omega=0$ in the zero temperature limit as shown in Fig. 4(c). While the imaginary part of local dynamical susceptibility, i.e., $\chi^{\prime \prime}(\omega) \propto \operatorname{Im} \chi^{(2)}(\omega)$ behaviors like a smoothness function $\sim \tanh \omega$ as given in Eq. (3.56) and shown in Fig. 4(d), which is expected to be a step function jumping at $\omega=0$ in the zero temperature limit.

\section{2. $\Delta=1 / 3: F F L$}

In this section, we study the spectral functions of a specific Schwarzian liquid with a conformal dimension $\Delta=1 / 3$. This is an intriguing phase between Schwarzian MFL phase $(\Delta=1 / 4)$ and Schwarzian FL $(\Delta=1 / 2)$ as will be discussed in more detail in the following section.

In Figs. 5(a)-5(b), we show the retarded Green's functions of FFL for $\Delta=1 / 3$ case with or without the Schwarzian correction, and we also plot the corresponding local dynamical susceptibility in Fig. 5(c)-5(d), which characters the local spin-spin correlation of disordered state.

\section{3. $\Delta=1 / 2$ : FL like}

The Fig. 6(a) shows the evolution of retarded Green's function with respect to the coupling coefficient $C^{-1}$ (where $C \sim C_{g} \bar{\phi}_{r}$ ), for FL (with $\Delta=1 / 2$ ) with Schwarzian correction, in terms of "Schwarzian FL". The coupling coefficient $C^{-1}$ (where $C \sim C_{g} \bar{\phi}_{r}$ ) characterizes the coupling

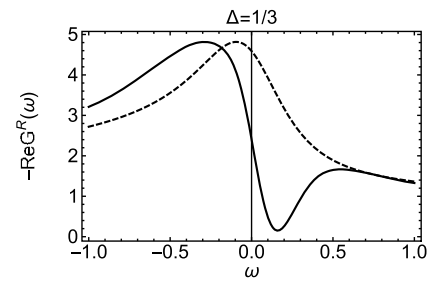

(a) $-\operatorname{Re} G^{R}(\omega)$

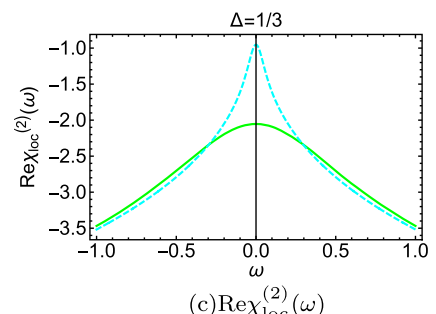

(c) $\operatorname{Re} \chi_{\operatorname{loc}}^{(2)}(\omega)$

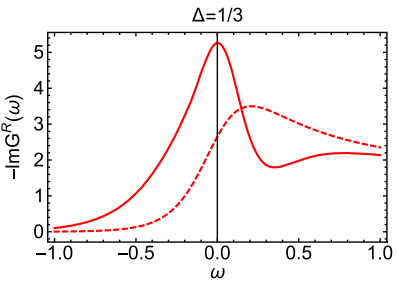

(b) $-\operatorname{Im} G^{R}(\omega) \sim$ DOS

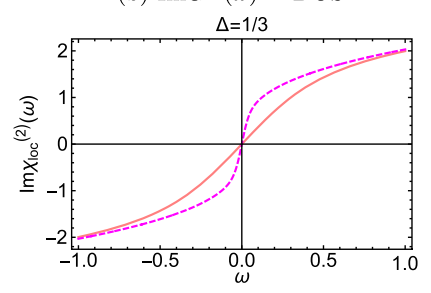

$(\mathrm{d}) \operatorname{Im} \chi_{\operatorname{loc}}^{(2)}(\omega)$
FIG. 5. Susceptibility or thermal retarded Green's functions $\chi(\omega)=G^{R}(\omega)$ of the quantum liquid with (solid line) or without (dashed line) the Schwarzian correction as given in Eq. (3.39). For $\Delta=1 / 3$ case: (a)-Re $G^{R}(\omega)$ (black line); (b)-Im $G^{R}(\omega)$ (red line). We have chosen $\beta=20 \pi$ and $C=1 /(2 \pi)$. Local dynamical spin-spin correlation functions of quantum liquid $\chi_{\text {loc }}^{(2)}(\omega)$ as given in Eq. (3.51): (c) $\operatorname{Re} \chi_{\mathrm{loc}}^{(2)}(\omega)$, (d) $\operatorname{Im} \chi_{\mathrm{loc}}^{(2)}(\omega)$ at high temperature with $\beta=2 \pi$ (solid green/pink line) or at low temperature with $\beta=20 \pi$ (dashed cyan/magenta line). strength of pNGBs loop corrections to matter two-point correlation functions, according to Eq. (2.47). The $C=\infty$ case (dotted red curves) corresponds to conventional FL, and the local susceptibility becomes exact step function $\theta(\omega)$ in the limit $\epsilon \rightarrow 0$. With the increasing of the coupling strength (or decreasing of $C$ ) up to $C^{-1}=3 \pi$ (solid purple line), the DOS accumulates more in the $\omega=0$ region and develops a small dig at the $\omega \approx 0.5$.

In Fig. 6(b), we also show the evolution of retarded Green's functions of Schwarzian FL with respect to the temperature. By decreasing temperature (or by increasing $\beta)$ from $T=1 /(2 \pi)$ down to $T=1 /(20 \pi)$ as well as $T=$ $1 /(200 \pi)$ (black/red dotted line). As expected, the DOS spread out among the frequency space at finite temperature, but there is still a peak at $\omega=0$, and a plateau in the $\omega>0$ region at low temperature limit as $T \rightarrow 0$.

\section{Large $p$ or small $\Delta$ behavior}

In this section, we consider the physical consequence when $\Delta$ becomes smaller as shown in Fig. 7. This is equivalent to increasing the number of interacting particles, i.e., $p \equiv 1 / \Delta[5])$. As stated before in the introduction section, the $p$-fermion interacting vertex with $p \geq 4$ is only UV relevant in $(0+1)$-dimensional spacetime. Without loss of generality, we chose some specific value for conformal dimension as $\Delta=1 / 8, / 16,1 / 32,1 / 64$, respectively. For larger $p$ or smaller $\Delta$, the spectral functions $\operatorname{Im} G^{R}(\omega)$ becomes more sharper at $\omega=0$. Consequently,
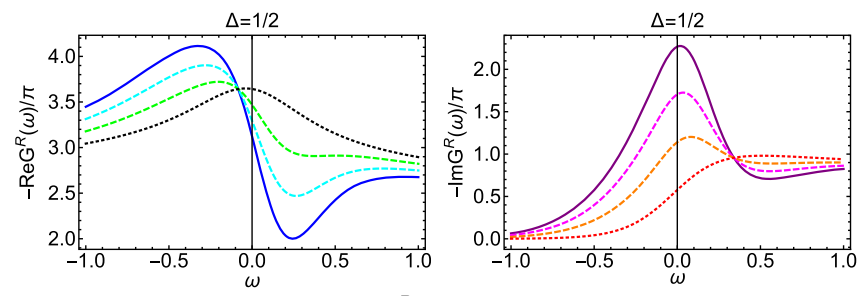

(a)Evolution of $\bar{G}^{R}(\omega)$ with coupling $(2 \pi C)^{-1}$
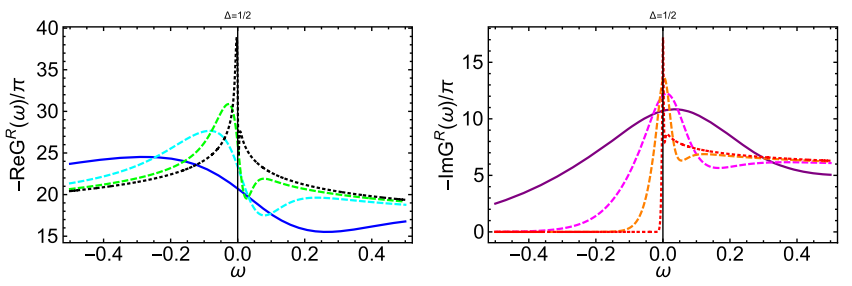

(b)Evolution of $\bar{G}^{R}(\omega)$ with temperature $\beta^{-1}$

FIG. 6. Dynamical susceptibility or retarded Green's functions of Schwarzian FL with $\Delta=1 / 2: \chi(\omega)=-\bar{G}^{R}(\omega) / \pi$ is given in Eq. (3.39). (a) Evolution with different coupling strength $(2 \pi C)^{-1}: C=1 / 3 \pi$ (blue/purple solid lines), $C=1 / 2 \pi$ (cyan/ magenta dashed line); $C=1 / \pi$ (green/orange dashed line) and $C=+\infty$ (black/red dotted line). We have chosen input parameters as $\beta=2 \pi$. (b) Evolution with different temperature $T$ : In front of Eq. (3.39), we have multiplying a temperature depending factor $\pi / \beta$. For different $T=\beta^{-1}: \beta=2 \pi$ (blue/purple solid lines), $\beta=20 \pi / 3$ (cyan/magenta dashed line); $\beta=20 \pi$ (green/ orange dashed line); $\beta=200 \pi$ (black/red dotted line). 


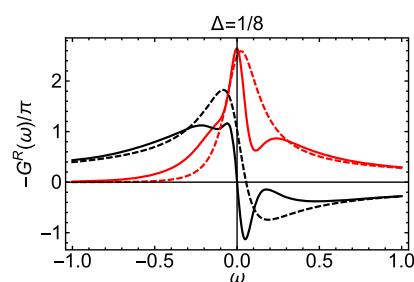

(a) $\Delta=1 / 8$

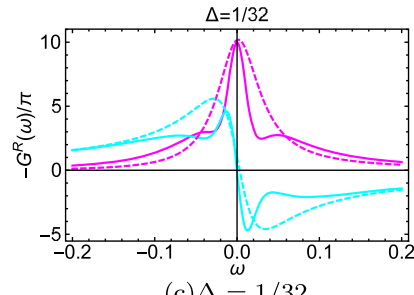

(c) $\Delta=1 / 32$

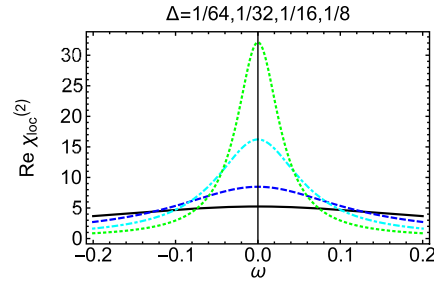

(e) $\operatorname{Re} \chi_{\text {loc }}$ (b) $\Delta=1 / 16$

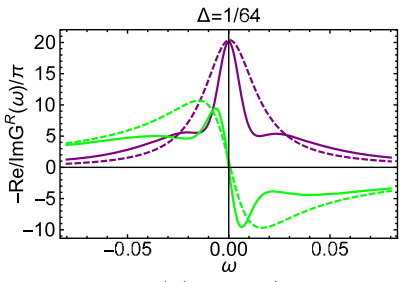

(d) $\Delta=1 / 64$

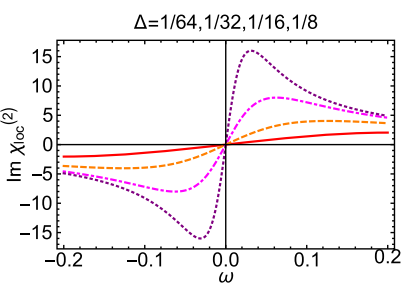

(f) $\operatorname{Im} \chi_{\text {loc }}$

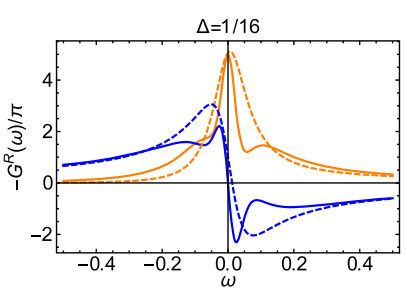

FIG. 7. Retarded Green's functions $G^{R}(\omega)$ of quantum liquid with (solid line) or without (dashed line) Schwarzian correction as given in Eq. (3.39). $\operatorname{Re} / \operatorname{Im} G^{R}(\omega)$ for (a) $\Delta=1 / 8$ (black or red line); (b) $\Delta=1 / 16$ (blue or orange line); (c) $\Delta=1 / 32$ (cyan or magenta line); (d) $\Delta=1 / 64$ (green or purple line). For all six cases, there are clean signatures of Hubbard band in DOS. Local dynamical susceptibility $\chi^{(2)}(\omega)$ with different $p \equiv 1 / \Delta(p=8$, 16, 32, 64 corresponds to solid, dashed, dot-dashed and dotted lines, respectively): (e) $\operatorname{Re} \chi^{(2)}(\omega)$; (f) $\operatorname{Im} \chi^{(2)}(\omega)$. We have chosen input parameters as $\beta=2 \pi$ and $C=1 /(2 \pi)$. With the increase of $p$, the density spectral function becomes more central localized at low frequency region, i.e., $\omega \sim 0$. the life time of the quasiparticle becomes longer as shown in Fig. 7. Conversely, for smaller $p$ or larger $\Delta$, the life time becomes shorter and the DOS shows nonquasiparticle behavior at low frequency.

With the increasing of $p=\Delta^{-1}$, or the decreasing of conformal dimension $\Delta$ from $\Delta=1 / 8$ to $\Delta=1 / 64$, the spectral functions $\operatorname{Im} G^{R}(\omega)$ become more and more centralized at $\omega=0$. It shows more metallic behavior at low frequency region, meanwhile the depth of Hubbard band increases towards low frequency region, and so does the location of Hubbard band.

\section{HIGH-POINT CORRELATION FUNCTIONS}

In this section, based on the low-energy effective action of the Schwarzian theory of time reparametrization from two-dimensional gravity, we calculate the high-point functions, especially the four-point correlation function $[51,57]$. The physical consequence of the four-point functions can be detected in the system of quantum chaos $[11,15,28,34,58-60]$, which can be characterized by an exponential growth of the thermal out-of-time-order correlating (OTOC) [59-64] four-point function with a scrambling time $\hat{t}$.

\section{A. Three-point function}

The three-point function $\left\langle\mathcal{O}^{\Delta}\left(t_{1}\right) \mathcal{O}^{\Delta}\left(t_{2}\right) \mathcal{O}^{\Delta}\left(t_{3}\right)\right\rangle$ in Eq. (2.50) can be generalized to a more generic case when $\Delta_{1}=\Delta_{2} \neq \Delta_{3}$, e.g., by introducing a bulk cubic interacting term $\Phi^{2} \phi$, coupled with two scalar and a dilaton, which is dual to boundary operators $\mathcal{O}_{\Phi}, \mathcal{O}_{\phi}$ with scaling dimension $\Delta$ and $\Delta_{\phi}=-1$, respectively [7]. To be more general, one can generalize the three-point functions to be $\left\langle\mathcal{O}^{\Delta_{1}}\left(t_{1}\right) \mathcal{O}^{\Delta_{2}}\left(t_{2}\right) \mathcal{O}^{\Delta_{3}}\left(t_{3}\right)\right\rangle$ as below

$$
G_{3}\left(t_{1}, t_{2}, t_{3}\right)=\frac{C_{3}}{\left[2 \sin \left(\frac{t_{12}}{2}\right)\right]^{\Delta_{12}}\left[2 \sin \left(\frac{t_{23}}{2}\right)\right]^{\Delta_{23}}\left[2 \sin \left(\frac{t_{13}}{2}\right)\right]^{\Delta_{13}}} \rightarrow \frac{C_{3}(\pi / \beta)^{\Delta_{12}+\Delta_{23}+\Delta_{13}}}{i^{\Delta_{123}}\left(\sinh \frac{\pi t_{12}}{\beta}\right)^{\Delta_{12}}\left(\sinh \frac{\pi t_{23}}{\beta}\right)^{\Delta_{23}}\left(\sinh \frac{\pi t_{13}}{\beta}\right)^{\Delta_{13}}},
$$

where $\Delta_{i j} \equiv \Delta_{i}+\Delta_{j}-\Delta_{k}$ and we have made the notation $\Delta_{123} \equiv \Delta_{12}+\Delta_{23}+\Delta_{13}=\Delta_{1}+\Delta_{2}+\Delta_{3}$. The generic coefficient in front turns out to be [65]

$$
C_{3}=-\frac{\Gamma\left(\frac{\left(\Delta_{123}-1\right)}{2}\right) \Gamma\left(\frac{\Delta_{12}}{2}\right) \Gamma\left(\frac{\Delta_{13}}{2}\right) \Gamma\left(\frac{\Delta_{23}}{2}\right)}{2 \pi \Gamma\left(\Delta_{1}-\frac{1}{2}\right) \Gamma\left(\Delta_{2}-\frac{1}{2}\right) \Gamma\left(\Delta_{3}-\frac{1}{2}\right)} .
$$

In the last equality, we have changed from the Euclidean/imaginary time to Loreantzian/real time, i.e., $t \rightarrow i t$. In the zero temperature limit, it recovers $G_{3}\left(t_{1}, t_{2}, t_{3}\right)=C_{3} /\left(t_{12}^{\Delta_{12}} t_{23}^{\Delta_{23}} t_{13}^{\Delta_{13}}\right)$. By doing an Fourier transform upon $\left(t_{1}, t_{2}, t_{3}\right)$ coordinate, one obtains the three-point correlation function in momentum space as

$$
G_{3}\left(\omega_{1}, \omega_{2}, \omega_{3}\right)=\int \prod_{i=1}^{3} d t_{i} e^{-i \omega_{i} t_{i}} G_{3}\left(t_{1}, t_{2}, t_{3}\right)=\int \frac{d t_{3} e^{-i\left(\omega_{1}+\omega_{2}+\omega_{3}\right) t_{3}} d t_{1} d t_{2} e^{-i\left(\omega_{2} t_{23}+\omega_{1} t_{13}\right)} C_{3}(\pi / \beta)^{\Delta_{123}}}{\left(\sinh \frac{\pi\left(t_{13}-t_{23}\right)}{\beta}\right)^{\Delta_{12}}\left(\sinh \frac{\pi t_{23}}{\beta}\right)^{\Delta_{23}}\left(\sinh \frac{\pi t_{13}}{\beta}\right)^{\Delta_{13}}}
$$

After integrating out $t_{3}$, one obtains a delta function $2 \pi \delta\left(\omega_{1}+\omega_{2}+\omega_{3}\right)$. By integrating out $\omega_{3}$ again, it becomes 


$$
G_{3}\left(\omega_{1}, \omega_{2}\right) \equiv \int \frac{d \omega_{3}}{2 \pi} G_{3}\left(\omega_{1}, \omega_{2}, \omega_{3}\right)=\int \frac{d s_{1} d s_{2} e^{-i\left(\omega_{2} s_{2}+\omega_{1} s_{1}\right)} C_{3}(\pi / \beta)^{\Delta_{123}}}{\left(\sinh \frac{\pi s_{12}}{\beta}\right)^{\Delta_{12}}\left(\sinh \frac{\pi s_{2}}{\beta}\right)^{\Delta_{23}}\left(\sinh \frac{\pi s_{1}}{\beta}\right)^{\Delta_{13}}},
$$

where we have redefined $t_{13}=s_{1}, t_{23}=s_{2}$ and it is worthy of noticing that $s_{12}=s_{1}-s_{2}$ is independent of $t_{3}$. For extremal case $\Delta_{3}=\Delta_{1}+\Delta_{2}$, one has $\Delta_{12}=0, \Delta_{23}=2 \Delta_{2}, \Delta_{13}=2 \Delta_{1}$, the three-point simplifies to be a product of 2 two-point correlation functions as

$$
G_{3}\left(\omega_{1}, \omega_{2}, \omega_{3}\right)=2 \pi \delta\left(\omega_{1}+\omega_{2}+\omega_{3}\right) C_{3} \int_{0}^{\beta} d t_{23} e^{-i \omega_{2} t_{23}} \frac{(\pi / \beta)^{2 \Delta_{2}}}{\left(\sinh \frac{\pi 2_{23}}{\beta}\right)^{2 \Delta_{2}}} \int_{0}^{\beta} d t_{13} e^{-i \omega_{1} t_{13}} \frac{(\pi / \beta)^{2 \Delta_{1}}}{\left(\sinh \frac{\pi t_{13}}{\beta}\right)^{2 \Delta_{1}}} .
$$

Alternatively, one may observe that assuming that the time translational invariance is kept, one can make a shift relative to $t_{13}$, so that the three-point correlation function in Eq. (4.1) can be reexpressed as

$$
G_{3}\left(t_{1}, t_{2}, t_{3}\right)=G_{3}\left(t_{12}, t_{23}, 0\right) \equiv G_{3}\left(s_{1}, s_{2}\right)=\frac{C_{3}(\pi / \beta)^{\Delta_{12}+\Delta_{23}+\Delta_{13}}}{i^{\Delta_{123}}\left(\sinh \frac{\pi s_{12}}{\beta}\right)^{\Delta_{12}}\left(\sinh \frac{\pi s_{2}}{\beta}\right)^{\Delta_{23}}\left(\sinh \frac{\pi s_{1}}{\beta}\right)^{\Delta_{13}}},
$$

we have redefined $t_{13} \equiv s_{1}, t_{23} \equiv s_{2}$ so that $t_{12}=t_{13}-t_{23}=s_{1}-s_{2}=s_{12}$. In this, case, we only need to make the Fourier transformation in $\left(s_{1}, s_{2}\right)$ coordinate and the same formula in Eq. (4.4), which can be reexpressed as

$$
G_{3}\left(\omega_{1}, \omega_{2}\right)=\int d s_{1} d s_{2} e^{-i\left(\omega_{2} s_{2}+\omega_{1} s_{1}\right)} \int d t \delta\left(t-s_{12}\right) \frac{C_{3}(\pi / \beta)^{\Delta_{12}+\Delta_{23}+\Delta_{13}}}{\left(\sinh \frac{\pi t}{\beta}\right)^{\Delta_{12}}\left(\sinh \frac{\pi s_{2}}{\beta}\right)^{\Delta_{23}}\left(\sinh \frac{\pi s_{1}}{\beta}\right)^{\Delta_{13}}},
$$

where we have introduced a delta function $\delta\left(t-s_{12}\right)=\int d \omega / 2 \pi e^{-i \omega\left(t-s_{12}\right)}$. The three-point function in momentum spacetime can be expressed in a convolution integral form of three two-point correlation as

$$
\begin{aligned}
G_{3}\left(\omega_{1}, \omega_{2}\right) & =\int d t \int \frac{d \omega}{2 \pi} e^{-i \omega\left(t-s_{12}\right)} \int d s_{1} d s_{2} e^{-i\left(\omega_{2} s_{2}+\omega_{1} s_{1}\right)} \frac{C_{3}(\pi / \beta)^{\Delta_{12}+\Delta_{23}+\Delta_{13}}}{\left(\sinh \frac{\pi t}{\beta}\right)^{\Delta_{12}}\left(\sinh \frac{\pi s_{2}}{\beta}\right)^{\Delta_{23}}\left(\sinh \frac{\pi s_{1}}{\beta}\right)^{\Delta_{13}}} \\
& =C_{3} \int \frac{d \omega}{2 \pi} \int d t \frac{e^{-i \omega t}(\pi / \beta)^{\Delta_{12}}}{\left(\sinh \frac{\pi(t-i \epsilon)}{\beta}\right)^{\Delta_{12}}} \int d s_{1} \frac{e^{-i\left(\omega_{1}-\omega\right) s_{1}}(\pi / \beta)^{\Delta_{13}}}{\left(\sinh \frac{\pi\left(s_{1}+i \epsilon\right)}{\beta}\right)^{\Delta_{13}}} \int d s_{2} \frac{e^{-i\left(\omega_{2}+\omega\right) s_{2}}(\pi / \beta)^{\Delta_{23}}}{\left(\sinh \frac{\pi\left(s_{2}+i \epsilon\right)}{\beta}\right)^{\Delta_{23}}} \\
& =C_{3} \int \frac{d \omega}{2 \pi} G_{\frac{\Delta_{12}}{2}}^{-}(\omega) G_{\frac{\Delta_{1}}{2}}^{+}\left(\omega_{1}-\omega\right) G_{\frac{\Delta_{2}}{2}}^{+}\left(\omega_{2}+\omega\right),
\end{aligned}
$$

the integrand above is nothing but the two-point correlation functions with conformal dimensions $\left(\Delta_{12}, \Delta_{13}, \Delta_{23}\right)$. In the last equality, we have used the Fourier transformation for the two-point correlation function as

$$
G_{\Delta}^{ \pm}(\omega) \equiv \int_{-\infty}^{+\infty} d \omega e^{-i \omega t}\left(\frac{\pi / \beta}{\sinh \left(\frac{\pi}{\beta}(t \pm i \epsilon)\right)}\right)^{2 \Delta}=e^{\mp i \pi \Delta} e^{\mp \frac{\beta}{2} \omega}\left(\frac{2 \pi}{\beta}\right)^{2 \Delta-1} \frac{1}{\Gamma(2 \Delta)}\left|\Gamma\left(\Delta-i \frac{\beta}{2 \pi} \omega\right)\right|^{2} .
$$

At zero temperature limit $(\beta \rightarrow \infty)$, it just recovers

$$
G_{3}\left(\omega_{1}, \omega_{2}\right)=C_{3} \int \frac{d \omega}{2 \pi} \int d t \frac{e^{-i \omega t}}{(t-i \epsilon)^{\Delta_{12}}} \int d s_{1} \frac{e^{-i\left(\omega_{1}-\omega\right) s_{1}}}{\left(s_{1}+i \epsilon\right)^{\Delta_{13}}} \int d s_{2} \frac{e^{-i\left(\omega_{2}+\omega\right) s_{2}}}{\left(s_{2}+i \epsilon\right)^{\Delta_{23}}} .
$$

By using Eq. (4.12), the retarded three-point Green's functions is defined as

$$
\begin{aligned}
G_{3}^{R}\left(\omega_{1}, \omega_{2}\right) & =C_{3} \int \frac{d \omega}{2 \pi} \int d t \theta(t) \frac{e^{-i \omega t}(\pi / \beta)^{\Delta_{12}}}{i^{\Delta_{12}}\left(\sinh \frac{\pi t}{\beta}\right)^{\Delta_{12}}} \int d s_{1} \theta\left(-s_{1}\right) \frac{e^{-i\left(\omega_{1}-\omega\right) s_{1}}(\pi / \beta)^{\Delta_{13}}}{i^{\Delta_{13}}\left(\sinh \frac{\pi s_{1}}{\beta}\right)^{\Delta_{13}}} \int d s_{2} \theta\left(-s_{2}\right) \frac{e^{-i\left(\omega_{2}+\omega\right) s_{2}}(\pi / \beta)^{\Delta_{23}}}{i^{\Delta_{23}}\left(\sinh \frac{\pi s_{2}}{\beta}\right)^{\Delta_{23}}} \\
& =C_{3} \int \frac{d \omega}{2 \pi} G_{\frac{\Delta_{12}}{2}}^{R}(\omega) G_{\frac{\Delta_{13}}{2}}^{A}\left(\omega_{1}-\omega\right) G_{\frac{\Delta_{23}}{2}}^{A}\left(\omega_{2}+\omega\right),
\end{aligned}
$$

where $G^{R} / G^{A}$ are retarded/advanced two-point Green's function in momentum spacetime as Eq. (4.12) is 


$$
G_{\Delta}^{R}(\omega) \equiv \int_{0}^{+\infty} d t e^{-i \omega t} \frac{(\pi / \beta)^{2 \Delta}}{\left(\sinh \frac{\pi t}{\beta}\right)^{2 \Delta}}=\left(\frac{2 \pi}{\beta}\right)^{2 \Delta-1} \Gamma(1-2 \Delta) \frac{\Gamma\left(\Delta+i \frac{\beta}{2 \pi} \omega\right)}{\Gamma\left(1-\Delta+i \frac{\beta}{2 \pi} \omega\right)}=G_{\Delta}^{A \star}(\omega) .
$$

In this case, the retarded three-point correlation function is

$$
\begin{aligned}
\left\langle G_{3}^{R}\left(\omega_{1}, \omega_{2}\right)\right\rangle= & C_{3} \int \frac{d \omega}{2 \pi}\left(\frac{2 \pi}{\beta}\right)^{\Delta_{12}+\Delta_{13}+\Delta_{23}-3} \Gamma\left(1-\Delta_{12}\right) \Gamma\left(1-\Delta_{13}\right) \Gamma\left(1-\Delta_{23}\right) \\
& \times \frac{\Gamma\left(\frac{\Delta_{12}}{2}+i \frac{\beta}{2 \pi} \omega\right)}{\Gamma\left(1-\frac{\Delta_{12}}{2}+i \frac{\beta}{2 \pi} \omega\right)} \frac{\Gamma\left(\frac{\Delta_{13}}{2}-i \frac{\beta}{2 \pi}\left(\omega_{1}-\omega\right)\right)}{\Gamma\left(1-\frac{\Delta_{13}}{2}-i \frac{\beta}{2 \pi}\left(\omega_{1}-\omega\right)\right)} \frac{\Gamma\left(\frac{\Delta_{23}}{2}-i \frac{\beta}{2 \pi}\left(\omega_{2}+\omega\right)\right)}{\Gamma\left(1-\frac{\Delta_{23}}{2}-i \frac{\beta}{2 \pi}\left(\omega_{2}+\omega\right)\right)} \\
= & C_{3}\left(\frac{2 \pi}{\beta}\right)^{\Delta_{123}-2} \Gamma\left(1-\Delta_{12}\right) \Gamma\left(1-\Delta_{13}\right) \Gamma\left(1-\Delta_{23}\right) G_{3,3}^{2,1}\left(1 \mid \begin{array}{c}
1-\frac{\Delta_{23}}{2}+i \frac{\beta}{2 \pi} \omega_{2}, 1-\frac{\Delta_{12}}{2}, 1-\frac{\Delta_{13}}{2}-i \frac{\beta}{2 \pi} \omega_{1} \\
\frac{\Delta_{12}}{2}, \frac{\Delta_{13}}{2}-i \frac{\beta}{2 \pi} \omega_{1}, \frac{\Delta_{23}}{2}+i \frac{\beta}{2 \pi} \omega_{2}
\end{array}\right),
\end{aligned}
$$

where $G_{p, q}^{m, n}$ are the Meijer $\mathrm{G}$ functions $G_{p, q}^{m, n}\left[\left\{\left\{a_{1}, \ldots, a_{n}\right\},\left\{a_{n+1}, \ldots, a_{p}\right\}\right\},\left\{\left\{b_{1}, \ldots, b_{m}\right\},\left\{b_{m+1}, \ldots, b_{q}\right\}\right\} ; z\right]$ with $n=1$, $m=2, p=3, q=3$ defined as below

$$
G_{p, q}^{m, n}\left(z \mid \begin{array}{l}
a_{1}, a_{2}, \cdots a_{p} \\
b_{1}, b_{2}, \cdots b_{p}
\end{array}\right) \equiv \frac{1}{2 \pi i} \int \frac{\prod_{j=1}^{m} \Gamma\left(b_{j}+s\right) \prod_{i=1}^{n} \Gamma\left(1-a_{i}-s\right)}{\prod_{k=n+1}^{p} \Gamma\left(a_{k}+s\right) \prod_{l=m+1}^{q} \Gamma\left(1-b_{l}-s\right)} z^{-s} d s,
$$

where we have defined $s=i \beta \omega / 2 \pi, z=1$, and

$$
\begin{aligned}
& b_{1}=\frac{\Delta_{12}}{2}, \quad b_{2}=\frac{\Delta_{13}}{2}-i \frac{\beta}{2 \pi} \omega_{1}, \quad 1-a_{1}=\frac{\Delta_{23}}{2}-i \frac{\beta}{2 \pi} \omega_{2}, \\
& a_{2}=1-\frac{\Delta_{12}}{2}, \quad a_{3}=1-\frac{\Delta_{13}}{2}-i \frac{\beta}{2 \pi} \omega_{1}, \quad 1-b_{3}=1-\frac{\Delta_{23}}{2}-i \frac{\beta}{2 \pi} \omega_{2} .
\end{aligned}
$$

Take $\Delta_{1}=\Delta_{2}=\Delta$ and $\Delta_{3}=\Delta_{h}$ as an example, the retarded three-point correlation function becomes

$$
\begin{aligned}
G_{\mathcal{O O}}^{R}\left(\omega_{1}, \omega_{2}\right)= & \frac{C_{3} \pi \Gamma\left(\Delta-\frac{i \beta \omega_{2}}{2 \pi}\right)_{3} F_{2}\left(2 \Delta-\Delta_{h}, \Delta+\frac{i \beta \omega_{1}}{2 \pi}, \Delta-\frac{i \beta \omega_{2}}{2 \pi} ; \Delta-\Delta_{h}+\frac{i \beta \omega_{1}}{2 \pi}+1, \Delta-\Delta_{h}-\frac{i \beta \omega_{2}}{2 \pi}+1 ; 1\right)}{\sin \left(\pi\left(\Delta_{h}-\Delta\right)-\frac{i \beta \omega_{1}}{2}\right) \Gamma\left(-2 \Delta+\Delta_{h}+1\right) \Gamma\left(-\Delta-\frac{i \beta \omega_{1}}{2 \pi}+1\right) \Gamma\left(\Delta-\Delta_{h}+\frac{i \beta \omega_{1}}{2 \pi}+1\right) \Gamma\left(\Delta-\Delta_{h}-\frac{i \beta \omega_{2}}{2 \pi}+1\right)} \\
& +\frac{C_{3} \pi \Gamma\left(\Delta_{h}-\frac{i \beta\left(\omega_{1}+\omega_{2}\right)}{2 \pi}\right)_{3} F_{2}\left(\Delta_{h}, \Delta-\frac{i \beta \omega_{1}}{2 \pi}, \Delta_{h}-\frac{i \beta \omega_{1}}{2 \pi}-\frac{i \beta \omega_{2}}{2 \pi} ;-\Delta+\Delta_{h}-\frac{i \beta \omega_{1}}{2 \pi}+1,-\frac{i \beta \omega_{1}}{2 \pi}-\frac{i \beta \omega_{2}}{2 \pi}+1 ; 1\right)}{\sin \left(\pi\left(\Delta-\Delta_{h}\right)+\frac{i \beta \omega_{1}}{2}\right) \Gamma\left(1-\Delta_{h}\right) \Gamma\left(-\Delta+\frac{i \beta \omega_{1}}{2 \pi}+1\right) \Gamma\left(1-\frac{i \beta\left(\omega_{1}+\omega_{2}\right)}{2 \pi}\right) \Gamma\left(-\Delta+\Delta_{h}-\frac{i \beta \omega_{1}}{2 \pi}+1\right)},
\end{aligned}
$$

where ${ }_{3} F_{2}\left(a_{1}, a_{2}, a_{3} ; b_{1}, b_{2} ; z\right)=\sum_{k=0}^{\infty}\left[\left(a_{1}\right)_{k}\left(a_{2}\right)_{k}\left(a_{3}\right)_{k} z^{k}\right] /\left[\left(b_{1}\right)_{k}\left(b_{2}\right)_{k} k !\right]$ is the generalized hypergeometric function with coefficients in terms of the Pochhammer symbol $(a)_{n}=\Gamma(a+n) / \Gamma(a)$.

\section{B. Four-point function}

\section{Zero-temperature case}

By using the correlation function $\mathcal{B}_{1}\left(t_{1}, t_{2}\right)$ in Eq. (2.36) and the soft mode propagators in Eq. (2.17), the correlation to the connected four-point function turns out to be $G^{(4)}\left(t_{1}, t_{2}, t_{3}, t_{4}\right)=\epsilon^{2}\left\langle\mathcal{B}_{1}\left(t_{12}\right) \mathcal{B}_{1}\left(t_{34}\right)\right\rangle /\left[t_{12} t_{34}\right]^{2 \Delta}$. For the normal ordering of the time case, i.e., $t_{1}>t_{2}>t_{3}>t_{4}$, the correlation function turns out to be vanishing,

$$
G_{\mathcal{V V W W}}^{(4)}=0
$$

which means that although the two-point function $\mathcal{B}_{1}\left(t_{12}\right)$ generates an energy fluctuation, they do not affect each other. While for the crossing time ordering case, i.e., $t_{1}>t_{3}>t_{2}>t_{4}$, the corresponding four-point function turns out to be nonvanishing. 


$$
G_{\mathcal{V W V W}}^{(4)}=\frac{1}{t_{12}^{2 \Delta} t_{34}^{2 \Delta}} \frac{\Delta^{2}}{9 C} \frac{t_{23}\left(t_{23}^{2}-3 t_{13} t_{24}\right)}{t_{12} t_{34}}
$$

which is proportional to the overall separation of the two pair $t_{23}$. In the absence of cross distance $t_{23}$, i.e., when $t_{2}=t_{3}$, the result $G_{\mathcal{V} \mathcal{W} \mathcal{V W}}^{(4)}$ just recovers $G_{\mathcal{V} \mathcal{V W W}}^{(4)}=0$. In the case $t_{3} \rightarrow t_{1}$ and $t_{2} \rightarrow t_{4}$, one has $G_{\mathcal{V W V W}}^{(4)} \sim t_{23}^{2} / t_{14}^{2} \sim 1$.

\section{Finite temperature case}

Suppose one has two operators $\mathcal{V}$ and $\mathcal{W}$ with the same conformal weight $\Delta$, which are dual to two bulk scalar field $\Phi$. The connected four-point function is given by

$$
\begin{aligned}
F^{(4)} & \equiv \frac{\left\langle\mathcal{V}\left(t_{1}\right) \mathcal{V}\left(t_{2}\right) \mathcal{W}\left(t_{3}\right) \mathcal{W}\left(t_{4}\right)\right\rangle}{\left\langle\mathcal{V}\left(t_{1}\right) \mathcal{V}\left(t_{2}\right)\right\rangle\left\langle\mathcal{W}\left(t_{3}\right) \mathcal{W}\left(t_{4}\right)\right\rangle}-1 \\
& =\epsilon^{2}\left\langle\mathcal{B}_{1}\left(t_{12}\right) \mathcal{B}_{1}\left(t_{34}\right)\right\rangle
\end{aligned}
$$

By using the correlation function $\mathcal{B}_{1}\left(t_{1}, t_{2}\right)$ in Eq. (2.42) and the soft mode propagators in Eq. (2.17), the correlation to the four-point function turns out to be $G^{(4)}\left(t_{1}, t_{2}, t_{3}, t_{4}\right)=$ $F^{(4)}\left(t_{1}, t_{2}, t_{3}, t_{4}\right) /\left[2 \sin \left(t_{12} / 2\right)\right]^{2 \Delta}\left[2 \sin \left(t_{34} / 2\right)\right]^{2 \Delta}$.

According to the relative ordering of the time, there are several possibilities; one is $t_{1}>t_{2}>t_{3}>t_{4}$, in which case one obtains

$$
F_{\mathcal{V V W W}}^{(4)}=\frac{2 \Delta^{2}}{\pi C}\left(1-\frac{t_{12}}{\tan \frac{t_{12}}{2}}\right)\left(1-\frac{t_{34}}{\tan \frac{t_{34}}{2}}\right),
$$

which can be viewed as arising from energy fluctuations. After recovering the thermal factor $t \rightarrow(2 \pi / \beta) t$, one just recovers the connected four-point function in Eq. (3.131) in Ref. [5]. Each two-point function $\mathcal{B}_{1}\left(t_{12}\right)$ generates an energy fluctuation, which affects each other. This result does not depend on the relative distance between the pair of points. In the double limit of $t_{12} \rightarrow 0$ and $t_{34} \rightarrow 0$, one has

$$
F_{\mathcal{V V W W}}^{(4)}=\frac{\Delta^{2}}{72 C \pi} t_{12}^{2} t_{34}^{2}
$$

The other result is obtained with the time order $t_{1}>t_{3}>t_{2}>t_{4}$, in this case, one has

$F_{\mathcal{V W V W}}^{(4)}=F_{\mathcal{V V W W W}}^{(4)}+\frac{\Delta^{2}}{C}\left(\frac{t_{23}}{\tan \frac{t_{12}}{2} \tan \frac{t_{34}}{2}}-2 \frac{\sin \frac{t_{23}}{2} \cos \frac{t_{14}}{2}}{\sin \frac{t_{12}}{2} \sin \frac{t_{34}}{2}}\right)$,

which depends on the overall separation of the two pair. In the absence of cross distance $t_{23}$, i.e., when $t_{2}=t_{3}$, the result $F_{\mathcal{V W} \mathcal{V W}}^{(4)}$ just recovers $F_{\mathcal{V} \mathcal{V W W}}^{(4)}$.

\section{OTOCS}

A simple diagnostic of quantum chaos is consider a square of the commutator by taking an expectation value in some thermal state, by considering a quantity, i.e., the commutator of operators separated in time as $[1,62]$

$$
\begin{aligned}
C(t) & =-\left\langle[\mathcal{V}(t), \mathcal{W}(0)]^{2}\right\rangle_{\beta}=C_{1}(t)-C_{2}(t), \\
C_{1}(t) & =\langle\mathcal{V}(t) \mathcal{W}(0) \mathcal{W}(0) \mathcal{V}(t)\rangle+\langle\mathcal{W}(0) \mathcal{V}(t) \mathcal{V}(t) \mathcal{W}(0)\rangle, \\
C_{2}(t) & =\langle\mathcal{V}(t) \mathcal{W}(0) \mathcal{V}(t) \mathcal{W}(0)\rangle+\langle\mathcal{W}(0) \mathcal{V}(t) \mathcal{W}(0) \mathcal{V}(t)\rangle,
\end{aligned}
$$

where $\mathcal{W}(t)$ and $\mathcal{V}(t)$ are two different operators dual to the source $\Phi_{0}(t)$, and $\langle\cdots\rangle_{\beta}=Z^{-1} \operatorname{Tr}\left[e^{-\beta H} \cdots\right]$, where the subscript $\beta$ is introduced to denote the thermal expectation value at temperature $T=\beta^{-1}$. The behavior of $C(t)$ in a chaotic system is by expanding it, there are four-point functions in $C$, two of them consist of $C_{1}(t)$ in terms of Lorentzian time-ordered correlators (TOCs), i.e., $G_{\mathcal{V} \mathcal{V W W}}^{(4)}=$ $\langle\mathcal{V}(t) \mathcal{V}(t) \mathcal{W}(0) \mathcal{W}(0)\rangle$, while the other two are OTOCs of the form $G_{\mathcal{V} \mathcal{W} \mathcal{W}}^{(4)}=\langle\mathcal{V}(t) \mathcal{W}(0) \mathcal{V}(t) \mathcal{W}(0)\rangle$, which can be used to diagnose chaos. The Feynman diagrams of fourpoint TOC and OTOC functions are shown in Fig. 8.

With the OTOC four-point function $G_{\mathcal{V W} \mathcal{V} \mathcal{W}}^{(4)}$, by making the parametrization with the SK four-contour [66,67], which is depicted in Fig. 9

$$
\left(\hat{t}_{i}\right)^{\text {OTOCs }}=\left(-\frac{\hat{t}}{2}-i \frac{\beta}{2}, \frac{\hat{t}}{2}-i \frac{\beta}{4},-\frac{\hat{t}}{2}, \frac{\hat{t}}{2}+i \frac{\beta}{4}\right),
$$

where $i=1,3,2,4$ and $\beta=2 \pi . \hat{t}$ is the separation of the early $V$ operator and the later $W$ operators. The contour goes from some initial time $\hat{t}_{1}$ within Euclidean domain, along the imaginary time axis to some time $\hat{t}_{2}$, then turns to the Euclidean domain time $\hat{t}_{3}$ again, and again runs along the imaginary time axis to $\hat{t}_{4}$.

In the $\hat{t} \gg \beta$ limit, one obtains the TOC and OTOC four-point functions, in the SK contour as depicted in Fig. 9
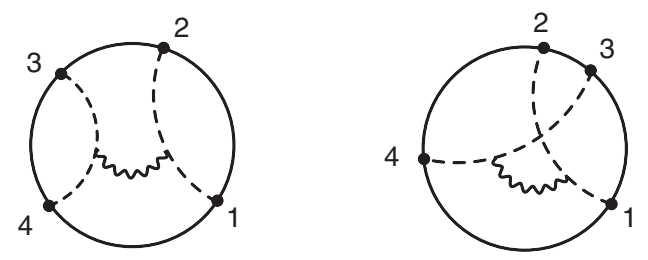

FIG. 8. Feynman diagrams for four-point correlation functions $G_{4}\left(t_{1}, t_{2}, t_{3}, t_{4}\right)$ of scalar fields with loop corrections from soft modes, as shown in Eq. (2.44). 


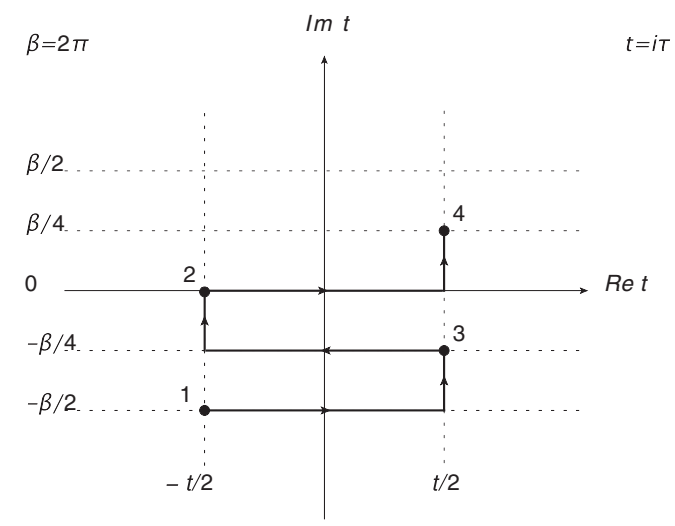

FIG. 9. Schwinger-Keldysh four contour for four-point OTOCs, with time chosen as in Eq. (4.24).

$$
\begin{aligned}
& G_{\mathcal{V} \mathcal{W} \mathcal{W}}^{(4)}=\frac{1}{4^{2 \Delta}} \frac{2 \Delta^{2}}{\pi C} \\
& G_{\mathcal{V W} \mathcal{W} \mathcal{W}}^{(4)}=-\frac{1}{4^{2 \Delta}} \frac{\Delta^{2}}{C} \cosh \hat{t} \sim \frac{1}{4^{2 \Delta}} \beta \frac{\Delta^{2}}{C} e^{\lambda_{L} \hat{t}},
\end{aligned}
$$

where $G_{\mathcal{V} \mathcal{V} \mathcal{W}}^{(4)}=G_{\mathcal{V} \mathcal{W} \mathcal{W}}^{(4)}\left(\hat{t}_{1}, \hat{t}_{2}, \hat{t}_{3}, \hat{t}_{4}\right)$ etc., and for the last equality of OTOCs, we have transferred the Euclidean time $\tau(t)$ in Eq. (2.7) to Minkowski time, i.e., $t_{i} \rightarrow i \hat{t}$ and recover the temperature by rescaling $t_{i} \rightarrow 2 \pi t_{i} / \beta$, while the $\beta$ in front comes from recovering of the thermal factor, i.e., by multiplying a factor $1 \rightarrow \beta / 2 \pi$. The behavior of thermal OTOCs at later time shows a exponential expansion with a Lyapnov exponent $\lambda_{L}=2 \pi / \beta$, which indicates the growth rate of chaos in thermal quantum systems with a large number of degrees of freedom, and is bounded in a universal system [59], as $\lambda \leq \lambda_{L}=2 \pi / \beta$. The Eq. (4.25) is valid under the condition that $t_{r} \ll t \ll t_{s}$, where $t_{r}$ is relaxation time, $t_{s}$ is the scrambling time and $t_{s} \sim \lambda_{L}^{-1} \ln C$ when $C(t)$ becomes of $O(1)$ under time long time evolution. By selecting the exponential increasing mode and doing Fourier transformation, in the choice of SK contour as in Eq. (4.24), or depicted in Fig. 9. The chaotic mode of OTOCs in frequency space becomes

$$
\begin{aligned}
G_{\mathcal{V W V W W}}^{(4)}(\omega) & =-i \frac{\beta}{2 \pi} \int_{-\infty}^{+\infty} d t e^{i \omega t} \theta(t) G_{\mathcal{V W} \mathcal{V W}}^{(4)}(t) \\
& =-\frac{\Delta^{2}}{4^{2 \Delta}} \frac{1}{2 C\left(\omega-i \lambda_{L}\right)}, \quad \operatorname{Im} \omega>\lambda_{L},
\end{aligned}
$$

while the normal mode of TOC becomes

$$
G_{\mathcal{V} \mathcal{V W W}}^{(4)}(\omega)=-\frac{1}{4^{2 \Delta}} \frac{\Delta^{2}}{C \omega}, \quad \operatorname{Im} \omega>0,
$$

which is singular at $\omega=0$. The chaotic behavior of fourpoint OTOC functions in frequency space is shown in Fig. 10. For maximal chaotic behavior with $\lambda_{L}=1$ $(\beta=2 \pi)$, it results in a nonzero frequency bump in the

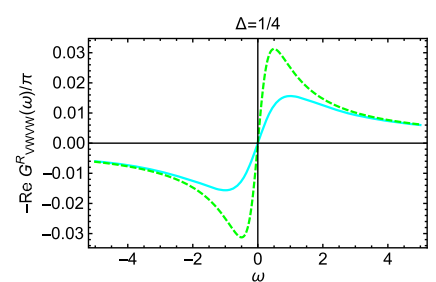

(a) $-\operatorname{Re} G_{\mathcal{V} \mathcal{W} \mathcal{V} \mathcal{W}}^{(4)}$

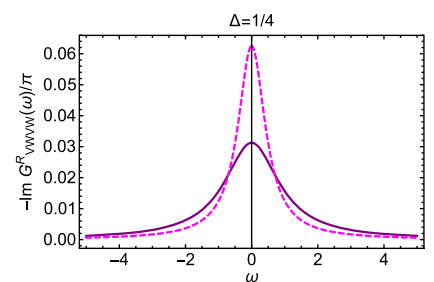

(b) $-\operatorname{Im} G_{\mathcal{V} \mathcal{W} \mathcal{V} \mathcal{W}}^{(4)} / \pi$
FIG. 10. The four-point OTOCs in frequency space with Lyapnov exponent as in Eq. (4.26) $-G_{\mathcal{V W} \mathcal{V W}}^{(4)}(\omega, \beta) / \pi$ of Schwarzian liquid: maximal chaotic behavior with $\lambda_{L}=1 \quad(\beta=2 \pi)$ (cyan/purple thick lines) or nonmaximal chaotic behavior with $\lambda_{L}=1 / 2(\beta=4 \pi)$ (green/magnet dashed lines). We have chosen a set of input parameters as $\Delta=1 / 4, C=1 /(2 \pi)$.

in low frequency region at large Lorentizian time. While at low temperature limit for $(\beta=4 \pi)$, the peak of the bulk moves more closer to low frequency range, or equivalently, a much more larger Lorentizian time to saturate the chaos, which corresponds to the nonmaximal chaotic behavior with $\lambda_{L}=1 / 2$. In the zero temperature limit, $\lambda_{L} \rightarrow 0$ $(\beta \rightarrow \infty)$, as expected, the peak of the bump moves to the $\omega=0$, and the mass spectrum of pNGB becomes NGB like.

\section{Six-point function}

It is straightforward to calculate the higher-point functions as in Eq. (2.43) such as the six-point functions obtained in Eq. (2.44). The typical Feynman diagrams of six-point TOC and OTOC functions are depicted in Fig. 11, respectively.

For the convenience of viewing physical consequence of six-point functions, one may generalize the SK fourcontour in Eq. (4.24) to be six-contour as shown in Fig. 12,
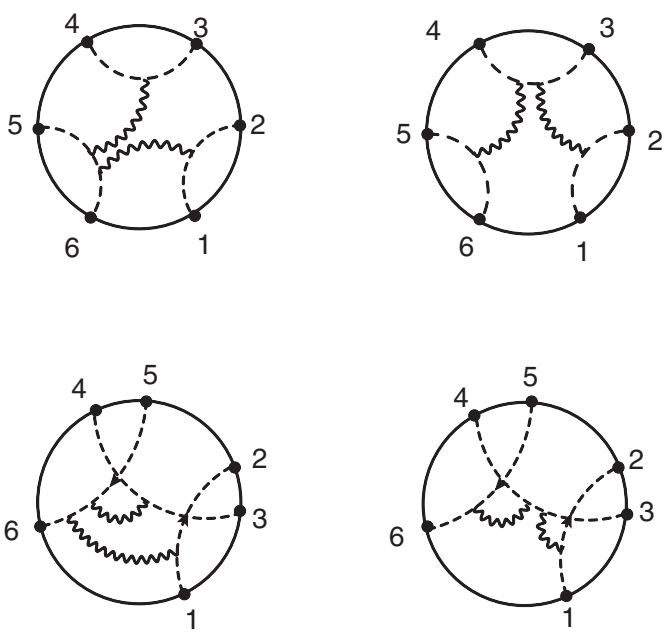

FIG. 11. Typical Feynman diagrams for six-point correlation functions $G_{6}\left(t_{1}, \ldots, t_{6}\right)$ of scalar fields with loop corrections from soft modes as shown in Eq. (2.44), in TOCs and OTOCs, respectively. 


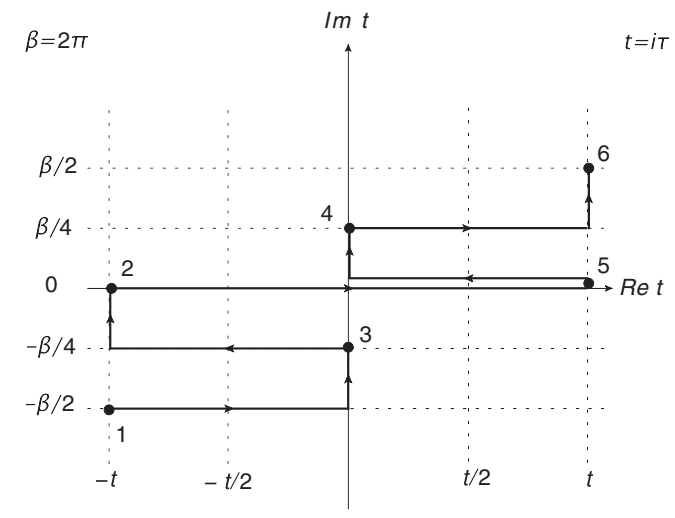

FIG. 12. Schwinger-Keldysh six contour for six-point OTOCs, with time chosen as in Eq. (4.28). One may entail an infinitesimal $\operatorname{Im} t_{52}=\delta>0$ to entail that $t_{2}$ is earlier than $t_{5}$ along imaginary time line, and in the end set it to be zero, which does not affect the results.

by increasing the real time with equal pace and imaginary time separately for OTOCs as

$$
\left(\hat{t}_{i}\right)^{\mathrm{OTOC}}=\left(-\hat{t}-\frac{\beta}{2} i,-\hat{t}-\frac{\beta}{4} i, 0,0+\epsilon i, \hat{t}+\frac{\beta}{4} i, \hat{t}+\frac{\beta}{2} i\right),
$$

where the time order is $i=1,3,2,5,4,6$.

For example, in the SK six contour chosen in Fig. 12, the six-point correlation function can be expressed more elegantly as $G_{6}\left(t_{1}, \ldots, t_{6}\right)=F_{6} / 4^{3 \Delta}$, where $F_{6}$ are

$$
\begin{aligned}
& F_{\mathcal{V W V X W X}}=F_{\mathcal{V W V W X X}}=\left(1-\frac{1}{2} \pi \cosh \hat{t}\right) F_{\mathcal{V N W W X X}}, \\
& F_{\mathcal{V V W W X X}}=F_{\mathcal{V} \mathcal{W W X W X}}=\frac{\left(8 \Delta+4-\pi^{2}\right) \Delta^{3}}{4 \pi^{2} C^{2}}
\end{aligned}
$$

where

$$
\begin{aligned}
& F_{\mathcal{V W V X \mathcal { W X }}}=\epsilon^{3}\left\langle\mathcal{B}_{1}\left(t_{1,2}\right) \mathcal{B}_{1}\left(t_{3,4}\right) \mathcal{B}_{2}\left(t_{5,6}\right) \theta\left(t_{32}\right) \theta\left(t_{54}\right)\right\rangle \\
& F_{\mathcal{V W V W X X}}=\epsilon^{3}\left\langle\mathcal{B}_{1}\left(t_{1,2}\right) \mathcal{B}_{1}\left(t_{3,4}\right) \mathcal{B}_{2}\left(t_{5,6}\right) \theta\left(t_{32}\right)\right\rangle \\
& F_{\mathcal{V V W} \mathcal{W} \mathcal{X X}}=\epsilon^{3}\left\langle\mathcal{B}_{1}\left(t_{1,2}\right) \mathcal{B}_{1}\left(t_{3,4}\right) \mathcal{B}_{2}\left(t_{5,6}\right) \theta\left(t_{54}\right)\right\rangle \\
& F_{\mathcal{V} \mathcal{W W} \mathcal{X X}}=\epsilon^{3}\left\langle\mathcal{B}_{1}\left(t_{12}\right) \mathcal{B}_{1}\left(t_{34}\right) \mathcal{B}_{2}\left(t_{56}\right)\right\rangle
\end{aligned}
$$

In a similar manner, one can redefine $\tilde{F}_{\mathcal{V} \mathcal{W} \mathcal{W X X}}=$ $F_{\mathcal{V N W W X X}}\left(t_{3,4} \leftrightarrow t_{5,6}\right)$ as

$$
\tilde{F}_{\mathcal{V} \mathcal{W W X X}}=\epsilon^{3}\left\langle\mathcal{B}_{1}\left(t_{12}\right) \mathcal{B}_{2}\left(t_{34}\right) \mathcal{B}_{1}\left(t_{56}\right)\right\rangle
$$

where $t$ is imaginary time, and $\theta\left(t_{i j}\right)$ is the step function. It turns out that, in the chosen SK six contour in Fig. (12), one obtains
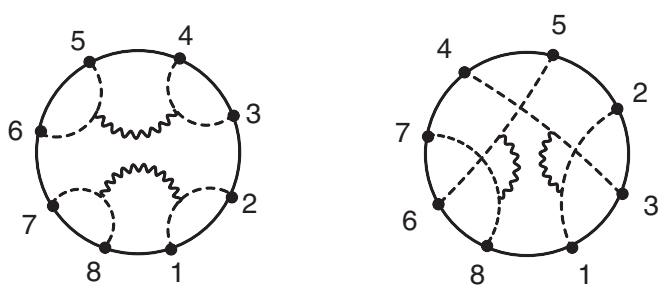

FIG. 13. Typical Feynman diagrams for eight-point functions $G_{8}\left(t_{1}, \ldots, t_{8}\right)$ of scalar fields with loop corrections from soft modes as shown in Eq. (2.44), in TOCs and OTOCs, respectively.

$$
\begin{aligned}
& \tilde{F}_{\mathcal{V W V X} \mathcal{X X}}-\tilde{F}_{\mathcal{V W V W X X}}-\tilde{F}_{\mathcal{V} \mathcal{W} \mathcal{X} \mathcal{W X}}+\tilde{F}_{\mathcal{V V W W X X}} \\
& =\frac{\Delta^{4}}{C^{2}} \cosh ^{2}(\hat{t}) \sim \frac{\Delta^{4}}{4 C^{2}} e^{2 \lambda_{L} \hat{t}},
\end{aligned}
$$

with the OTOCs time as $\hat{t}_{i}$ with $i=(1,3,2,5,4,6)$ and $\lambda_{L}=2 \pi / \beta$. The results just recover the 3-OTCs of sixpoint functions in Ref. [34].

\section{Eight-point function}

According to Eq. (2.43), the eight-point functions are obtained in Eq. (2.44). The typical Feynman diagrams of eight-point TOC and OTOC functions are depicted in Fig. 13, respectively.

For the convenience of viewing physical consequence of eight-point OTOC functions, one may generalize the SK four-contour in Eq. (4.24) to be eight-contour as shown in Fig. 14, by increasing the real time with equal pace and imaginary time separately, as

$$
\begin{aligned}
\left(\hat{t}_{i}\right)^{\mathrm{OTос}}= & \left(-\frac{3}{2} \hat{t}-\frac{\beta}{2} i,-\frac{1}{2} \hat{t}-\frac{\beta}{4} i ;-\frac{3}{2} \hat{t}, \frac{1}{2} \hat{t}+\epsilon i\right. \\
& \left.-\frac{1}{2} \hat{t}+\frac{\beta}{4} i, \frac{3}{2} \hat{t}+\frac{\beta}{4} i+\epsilon^{\prime} i ; \frac{1}{2} \hat{t}+\frac{\beta}{2} i, \frac{3}{2} \hat{t}+\frac{3 \beta}{4} i\right),
\end{aligned}
$$

where the time order is $i=(1,3,2,5,4,7,6,8)$.

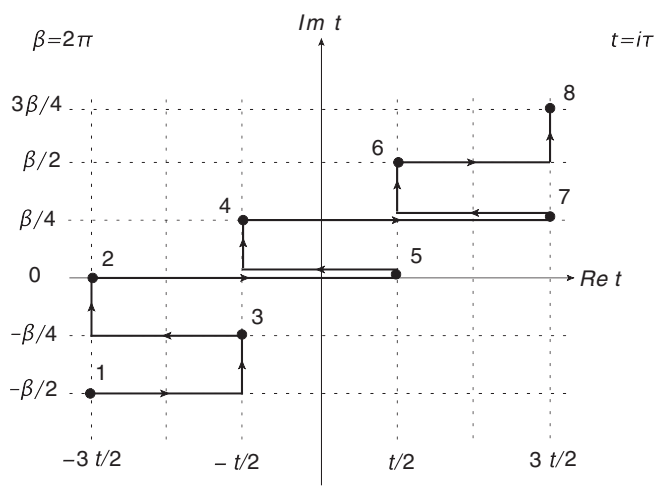

FIG. 14. Schwinger-Keldysh eight contour for eight-point OTOCs, with time chosen as in Eq. (4.33). One may entail infinitesimals $\operatorname{Im} t_{52}=\delta>0$ and $\operatorname{Im} t_{74}=\delta^{\prime}>0$ to entail that $t_{2,4}$ is earlier than $t_{5,7}$, respectively, along the imaginary time axis. 
In a similar manner as in calculating the six-point function, in the chosen SK eight-contour as in Fig. 14 with the OTOCs time as $\hat{t}_{i}$ with $i=(1,3,2,5,4,7,6,8)$, the thermal eight-point functions turns out to be $G_{8} \sim F_{8} / 4^{4 \Delta}$, where $F_{8}$ are

$$
\begin{aligned}
& F_{\mathcal{V} \mathcal{W W} \mathcal{X} X \mathcal{Y Y}}=F_{\mathcal{V} \mathcal{W} \mathcal{X} \mathcal{W} \mathcal{Y Y}}=\frac{4 \Delta^{4}}{\pi^{2} C^{2}}, \\
& F_{\mathcal{V W V Z W X Z X}}=F_{\mathcal{V W V W Z}}{ }_{\mathcal{X Z X}} \\
& =-F_{\mathcal{V W V Z W Z X X}}=-F_{\mathcal{V V W Z}}{ }_{\mathcal{W X Z X}} \\
& =-F_{\mathcal{V W V W Z Z X X}}=-F_{\mathcal{V V W W Z X Z X}} \\
& =\frac{4 \Delta^{4}}{\pi^{2} C^{2}}\left(1-\frac{\pi}{2} \cosh \hat{t}\right)^{2} \sim \frac{\Delta^{4} e^{2 \lambda_{L} \hat{t}}}{4 C^{2}},
\end{aligned}
$$

where

$$
\begin{aligned}
& F_{\mathcal{V U W W X X Y Y}}=\epsilon^{3}\left\langle\mathcal{B}_{1}^{4}\left(t_{1,8}\right)\right\rangle, \\
& F_{\mathcal{V V W X W X Y Y}}=\epsilon^{3}\left\langle\mathcal{B}_{1}^{4}\left(t_{1,8}\right) \theta\left(t_{54}\right)\right\rangle \text {, } \\
& F_{\mathcal{V W V Z W X Z X}}=\epsilon^{3}\left\langle\mathcal{B}_{1}^{4}\left(t_{1,8}\right) \theta\left(t_{32}\right) \theta\left(t_{54}\right) \theta\left(t_{76}\right)\right\rangle \text {, } \\
& F_{\mathcal{V W V W Z X Z X X}}=\epsilon^{3}\left\langle\mathcal{B}_{1}^{4}\left(t_{1,8}\right) \theta\left(t_{32}\right) \theta\left(t_{76}\right)\right\rangle, \\
& F_{\mathcal{V W V Z W Z X X}}=\epsilon^{3}\left\langle\mathcal{B}_{1}^{4}\left(t_{1,8}\right) \theta\left(t_{32}\right) \theta\left(t_{54}\right)\right\rangle, \\
& F_{\mathcal{V V W Z W X Z X}}=\epsilon^{3}\left\langle\mathcal{B}_{1}^{4}\left(t_{1,8}\right) \theta\left(t_{54}\right) \theta\left(t_{76}\right)\right\rangle, \\
& \left.F_{\mathcal{V W V W Z Z X X}}=\epsilon^{3}\left\langle\mathcal{B}_{1}^{4}\left(t_{1,8}\right) \theta\left(t_{32}\right)\right)\right\rangle \text {, } \\
& F_{\mathcal{V V W W Z X Z X}}=\epsilon^{3}\left\langle\mathcal{B}_{1}^{4}\left(t_{1,8}\right) \theta\left(t_{76}\right)\right\rangle,
\end{aligned}
$$

where we have introduced the new notation $\mathcal{B}_{1}^{4}\left(t_{1,8}\right) \equiv$ $\mathcal{B}_{1}\left(t_{1,2}\right) \mathcal{B}_{1}\left(t_{3,4}\right) \mathcal{B}_{1}\left(t_{5,6}\right) \mathcal{B}_{1}\left(t_{7,8}\right)$. It is easy to check that it satisfy the relation

$$
\begin{aligned}
& F_{\mathcal{V W V Z W Z X X}}-F_{\mathcal{V W V W Z Z X X}}-F_{\mathcal{V V W X}}{ }_{\text {WXYY }} \\
& +F_{\mathcal{V} \mathcal{W W X X Y Y Y}}=0
\end{aligned}
$$

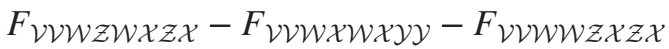

$$
\begin{aligned}
& +F_{\mathcal{V N W W X X Y Y}}=0, \\
& F_{\mathcal{V W V W Z X Z X}}-F_{\mathcal{V W V W Z Z X X}}-F_{\mathcal{V V W W Z X X X}} \\
& +F_{\mathcal{V} \mathcal{W W} \mathcal{X X Y \mathcal { Y }}}=\frac{\Delta^{4} \cosh ^{2}(\hat{t})}{C^{2}} \sim \frac{\Delta^{4} e^{2 \lambda_{L} \hat{t}}}{4 C^{2}} .
\end{aligned}
$$

For the higher-point OTCs, one would expect that the thermal system will approach the chaos much faster with time less than $\tau_{L} \equiv 1 / \lambda_{L}$.

\section{DISCUSSIONS AND CONCLUSION}

The SYK model is an intriguing quantum mechanical model displaying both a spontaneous and explicit breaking of an emergent reparametrization symmetry Diff ${ }_{1}$. The breaking patten of this symmetry determines many feature of the low-energy dynamical property of the model and some are expected to be universal in strongly interacting IR fixed point at large $\mathrm{N}$ limit.

\section{A. Features of the SYK-like model}

The most fabulous features of the SYK model is the solvability in the strongly interacting IR fixed point at large $\mathrm{N}$ limit. The mass spectrum of the SYK model is obtained by solving Schwinger-Dyson equation and the spectrum of two-point and four-point function, as well as more higherpoint functions are computed [3,5,32].

The other interesting features of the model is that in the strong coupling limit $(\beta J \gg 1)$, the four-point function saturates the maximal chaotic bound since it is dominated by the universal sector of gravity [59] which is characteristic of a gravity theory with black hole solutions [58]. The saturation means it achieves the maximally allowed chaos quantified by the Lyapunov exponent $\lambda=2 \pi \beta$, the growing rate of a thermal four-point OTOC function, as defined on the Keldysh contour [1,6], $\left\langle\mathcal{V}_{i}(0) \mathcal{W}_{j}(t) \mathcal{V}_{i}(0) \mathcal{W}_{j}(t)\right\rangle_{\beta} \sim e^{\lambda t} / N$, which is true at a time range between the dissipation time and the scrambling time, i.e., $t \in\left(\lambda^{-1}, \lambda^{-1} \ln N\right)$. The exponential growing manner reflects an underlying chaotic dynamics.

Another novel feature of the model is the emergent conformal symmetry, i.e., the time reparametrizations diffeomorphism symmetry Diff ${ }_{1}$, or Virasoro symmetry, at low energy and its spontaneous and explicit breaking [5,7].

\section{B. Spontaneous breaking of Diff 1}

In the SYK model, the emergent Diff ${ }_{1}$ symmetry is spontaneously broken down to $\operatorname{SL}(2, R)$ symmetry $[68,69]$, which is kept in the Schwarzian action (the Lagrangian) at finite frequency. From the gravity viewpoint, the Diff symmetry is an approximate asymptotic boundary symmetry of the perfect $\mathrm{AdS}_{2}$ at IR conformal fixed point $(\omega=0$ or $J=\infty)$, and is spontaneously broken down to a one-dimensional global conformal group $\mathrm{SO}(2,1) \sim$ $\mathrm{SL}(2, R)$ symmetry, or large diffeomorphism Diff owned by the $\mathrm{AdS}_{2}$ symmetry.

\section{Explicit breaking of Diff 1}

In the SYK model, the emergent Diff ${ }_{1}$ symmetry is also explicitly broken, since the symmetry is not kept by the Lagrangian any more as one slightly moves away from the IR conformal fixed point, where the kinetic term $\partial_{\tau}$ becomes relevant at low frequency or strong coupling region $(\omega \ll 1$ or $J \gg 1)$. From the gravity viewpoint, the bulk spacetime is slightly deviated from $\mathrm{AdS}_{2}$ vacuum to $\mathrm{NAdS}_{2}$ by taking account of the backreaction due to arbitrary tiny energy excitation.

\section{Diff $\boldsymbol{1}_{\mathbf{1}}$ symmetry breaking pattern}

The pattern of spontaneous breaking of the Diff ${ }_{1}$ results in an infinite number of zero mode, namely, the NGBs characterized by the coset $\operatorname{Diff}_{1} / \mathrm{SL}(2, R)$. As the $\operatorname{Diff}_{1}$ 
symmetry is explicitly broken, the leading-order dynamical correction is described by a $\mathrm{SL}(2, R)$-invariant Schwarzian derivative of the reparametrization $f(t)$ in terms of effective Schwarzian action in Eq. (A11) as described in Appendix A, which determines many aspects of the theory. As will be seen, the dynamics of the Schwarzian correction to the quantum correlations of SYK model is characterized by Schwarizian action with an $\operatorname{SL}(2, R)$ unbroken symmetry. As the Diff ${ }_{1}$ symmetry is explicitly breaking due to a small but nonvanishing derivation $\epsilon \sim \kappa \sim J^{-1}$ (this is equivalent to a small $\omega \ll 1$ or the presence of a relevant kinetic term $\partial_{\tau}$ ), associated with an infinitesimal fluctuation field $k(t)$ in a dynamical reparametrization function $f=t+\epsilon k(t)$, as in Eq. (2.7) and (2.13) for zero and finite temperature cases, respectively. To be brief, the Diff ${ }_{1}$ symmetry is parametrized by $f$ and is explicitly broken by the small fluctuation $\epsilon k(t)$, which is parametrized by the coset $\operatorname{Diff}_{1} / \mathrm{SL}(2, R)$.

\section{E. Quantum and thermal correlations}

For zero temperature case as in Eq. (2.10), the zero modes of the fluctuation field, i.e., NGBs, leads to a zero action in the IR conformal fixed point $(J=\infty$ or $\epsilon=0)$, while the soft modes of the fluctuation field, i.e., pNGBs, leads to a nonvanishing action when the classic solution is deviated away from the conformal limit (a small but finite $\epsilon \sim J^{-1}$ ). For finite temperature case as in Eq. (2.16), the first exciting state of pNGBs, i.e., the $n= \pm 1$ soft modes, also leads to a zero action. In both cases, the two-point function is singular and needs to be regularized. Consequently, the two-point and four-point correlation functions of matter field obtain loop corrections from the pNGBs as the reminiscent of the breaking of the time reparamterization symmetry $\operatorname{Diff}_{1}$.

In conclusion, we study the retarded Green's function of Schwarzian liquid, which can be depicted by a $(0+1)$ dimensional strongly interacting quantum mechanical/statistics model dual to a general $(1+1)$-dimensional classical dilaton gravity model. Based upon the two-point correlation functions of matter, which get loop corrections from pNGBs in coset $\operatorname{Diff}_{1} / \mathrm{SL}(2, R)$, we obtain the bosonic retarded Green's functions as well as local dynamical susceptibility, i.e., the second-order local spin-spin correlation functions for quantum liquid. We also calculate the four-point as well as higher-point thermal OTOC functions in SK formalism, which cultivate the quantum chaos at large real time.

To manifest our results, we show the spectral functions of not only Schwarzian MFL described with conformal dimension $\Delta=1 / 4(p=4)$ but also Schwarzian FL with $\Delta=1 / 2(p=2)$, as well as a specific quantum liquid phase with $\Delta=1 / 3(p=3)$. Large $p$-body behavior of the Schwarzian liquid is studied too. Moreover we make comparison with the leading-order retarded Green's functions, which just recovers the results of quantum liquid from $\mathrm{AdS}_{2} / \mathrm{CFT}_{1}$ approach.

In the infrared (IR) conformal fixed point with zero frequency $(\omega=0)$ where the Diff ${ }_{1}$ symmetry is emergent, the spectral functions owns Fermi liquid [70] peak in DOS at $\omega=0$ and leads to typical metallic behavior. The symmetry is spontaneously broken to $\operatorname{SL}(2, R)$ and leads to zero modes on the boundary in terms of "boundary graviton" [7], which are the Fourier modes of the Diff ${ }_{1}$ symmetry. At finite frequency $(\omega \neq 0)$, the Diff ${ }_{1}$ symmetry is explicit broken. As its physical consequence, the system develops a feature which is interpreted as bad metalic behavior with a high-energy Hubbard band dynamically generated. In the intermediate region, there is a temperature dependent crossover between Fermi liquid phase and bad metal phase in the strongly correlation regime, in which the quasiparticle picture is fragile or even broken down.

We make generalizations of four-point correlation to higher-point correlation functions. As noninclusive demos, we show concise analytic results on six-point as well as eight-point thermal OTOC functions in SK contour, which exhibit exponential growth until progressively a longer timescale and thus sensitive to more fine grained quantum chaos. We also obtain analytic expression for third-order and fourth-order local spin-spin correlation functions.

The Schwarzian liquid can be related not only to FFL, but also disordered metals without quasiparticles scenery depicted by SYK like model, such as NFL phase $[38,43,52-56]$. We study the matter retarded Green's function by taking account of the loop corrections from pNGBs to the matter two-point correlation function, and unexpectedly find a Hubbard band or dynamically generated DOS in the spectral functions, which is due to the spontaneous and explicit breaking of time reparametrization symmetry and is a distinct signature of Schwarzian liquid, comparing with the conventional strongly interacting quantum liquid. The existence of pNGBs mode from Schwarzian action in the quantum liquid also provides a dynamical mechanism for explaining the commonly observations of bad metal in strongly correlated system.

\section{ACKNOWLEDGMENTS}

For valuable discussions, we thank Gleb Arutyunov, Roberto Emparan, Ara Go, Sunly Khimphun, Ki-Seok Kim, Bum-Hoon Lee, Yue-Zhou Li, Andrew Lucas, Zhan-Feng Mai, Eun-Gook Moon, Ioannis Papadimitriou, Varun Sethi, Run-Qiu Yang, Hossein Yavartanoo, Junggi Yoon, Kentaroh Yoshida, and YunLong Zhang. This work is supported by the Mid-career Researcher Program through the National Research Foundation (NRF) of Korea under Grant No. NRF2016R1A2B3007687. Y.S. is supported by the Basic Science Research Program through NRF under Grant No. NRF-2016R1D1A1B03931443. For the hospitality, we also would like to thank APCTP focus workshop 
program "Geometry and Holography of Quantum Critical Point" during the visit at the POSTECH, Pohang. The authors thank the Yukawa Institute for Theoretical Physics at Kyoto University. Discussions during the workshop YITP-T-18-04 "New Frontiers in String Theory" were useful in completing this work.

\section{APPENDIX A: SYK MODEL AND SCHWARZIAN}

\section{The action of SYK model}

\section{a. SYK model introduction}

In the path integral, the prototypical SYK model is described by the partition function an action $Z(J)=$ $\int D \psi_{i} e^{-S}$, with the action as

$$
S=\int d \tau\left(\frac{1}{2} \sum_{i=1}^{N} \chi_{i} \partial_{\tau} \chi_{i}-\frac{1}{4 !} \sum_{i, j, k, l} J_{i j k l} \chi^{i} \chi^{j} \chi^{k} \chi^{l}\right),
$$

where $\chi^{i}$ are $N$ Majorana fermions, satisfying $\left\{\chi_{i}, \chi_{j}\right\}=$ $\delta_{i j}$, interacting with random interactions involving four fermions at a time. $J_{i j k l}$ is a Gaussian random infinite-range exchange interaction of all-to-all quartic coupling, which are mutually uncorrelated and satisfies the Gaussian's probability distribution function $P\left(J_{i j k l}\right) \sim \exp \left(-N^{3} J_{i j k l}^{2} /\right.$ $\left.12 J^{2}\right)$, which leads to zero mean $\mathbb{E}\left[J_{i j k l}\right]=0$ and variance $\mathbb{E}\left[J_{i j k l}^{2}\right]=3 ! J^{2} / N^{3}$ with width of order $J / N^{3 / 2}$, respectively. The $\mathbb{E}[\cdots]$ denotes an average over disorder. The $J$ is the only one effective coupling after the disorder averaging for the random coupling $J_{i j k l}$. The random couplings $J_{i j k l}$ represents disorder, and does not correspond to a unitary quantum mechanics [14,33]. For Euclidean time $\tau=i t$, the model can be viewed alternatively as a one-dimensional statistical model of Majorana fermions. For finite temperature case, the quantum mechanical model can be alternatively depicted in a quantum statistics. By using a Hubbard-Stratonovich transformation, it is possible to rewrite the original partition function of SYK model as a functional integral of the form [1,39-41] as

$Z=e^{-\beta F}=\int \mathcal{D} \mathcal{G} \mathcal{D} \Sigma \exp (-N \bar{S})$,

$\bar{S}=-\left[\ln \operatorname{Pf}\left(\partial_{\tau}-\Sigma\right)-\frac{1}{2} \int d \tau_{1} d \tau_{2}\left(\Sigma \mathcal{G}-\frac{J^{2}}{4} \mathcal{G}^{p}\right)\right]$,

where $\bar{S}=S / N$ is a disorder-averaged nonlocal effective action by doing Gaussian integral over the disorder and integrating out fermions after introducing a bilocal field $\mathcal{G}\left(\tau, \tau^{\prime}\right)$ and a Lagrange multiplier field $\Sigma\left(\tau, \tau^{\prime}\right)$. Pf denotes the Pfaffian, and the first term of the action can also be reexpressed as $\ln \left[\operatorname{det}\left(\partial_{\tau}-\Sigma\right)\right] / 2, \tau, \tau^{\prime}$ are Matsubara times, and $p=4$ denotes the number of Majorana fermion in the vertex.

At large $N$ limit, i.e, a model with the number of Majorana fermion $N \gg 1$, by doing variation with respect to $\mathcal{G}$ and $\Sigma$, or equivalently by counting the resummed Feynman diagrams, the solution of SYK model is described by the Schwinger-Dyson (SD) equations in real spacetime as

$$
\mathcal{G}=\left(\partial_{\tau}-\Sigma\right)^{-1}, \quad \Sigma=J^{2} \mathcal{G}^{p-1},
$$

where $\mathcal{G}=\mathcal{G}\left(\tau, \tau^{\prime}\right)$ is the two-point Green's function, $\Sigma=$ $\Sigma\left(\tau, \tau^{\prime}\right)$ is one particle irreducible (1PI) self-energy.

The first kinetic term in the $G$ represents a conformal breaking term as will be clear in the following. Substituting the full solutions of the above classical equations of motion back into the effective action in the partition functions, one obtains the leading large- $N$ saddle-point free energy $F$ in low temperature expansion as $[5,11]$

$$
\begin{aligned}
\bar{F} & =\frac{F}{N}=-\frac{1}{\beta} \frac{\ln Z}{N}=\frac{1}{\beta} \bar{S} \\
& =e_{0}-s_{0} \beta^{-1}-\frac{1}{2} \gamma \beta^{-2}+\cdots,
\end{aligned}
$$

where $e_{0}$ is the nonuniversal (UV completion sensitive) ground state energy density, $s_{0}$ is the universal zero temperature entropy density and $c_{v} \equiv \gamma \beta^{-1}$ is the specific heat density. $\cdots$ denote terms with higher order in $\beta^{-1}$.

The residual zero temperature entropy density and the thermal capacity density turns out to be

$$
\begin{aligned}
& s_{0}=-\frac{\partial \bar{F}}{\partial T}=-\frac{1}{T} \frac{\partial \bar{F}}{\partial \ln T}=\beta \frac{\partial \bar{F}}{\partial \ln \beta}=\beta^{2} \frac{\partial \bar{F}}{\partial \beta} \\
& c_{v}=\frac{1}{\beta} \gamma=-T \frac{\partial^{2} \bar{F}}{\partial^{2} T}=T \frac{\partial s_{0}}{\partial T}=\frac{\partial s_{0}}{\partial \ln T}=-\beta \frac{\partial s_{0}}{\partial \beta} .
\end{aligned}
$$

In the ultraviolet (UV) limit at short distance, $\omega \gg J$, the kinetic term dominates and the four-fermion interactions term is irrelevant so that the theory has $N$ weakly interacting massless Majorana fermions. The fermions have a two-point function given by $\mathcal{G}_{0}(\tau)=\operatorname{sgn}(\tau) / 2$ regardless of temperature, or $\mathcal{G}(\omega)=i \omega^{-1}$ in frequency space, assuming the time translation symmetry is kept. The action is invariant under arbitrary time reparametrizations and consequently the Hamiltonian is zero. While in the low-energy IR limit at large distance, the frequency (in the momentum spacetime $\left.\partial_{\tau} \sim i \omega\right)$ is much smaller than the UV coupling $J$, i.e., $\omega \ll J$, means the model becomes strongly interacting at low energies. Consequently, the kinetic term $\partial_{\tau}$ can be dropped, so that the SD equations in the IR limit are modified to be conformal invariant ones as in

$$
\int d \tau^{\prime \prime} \mathcal{G}\left(\tau, \tau^{\prime \prime}\right) \Sigma\left(\tau^{\prime \prime}, \tau^{\prime}\right)=-\delta\left(\tau-\tau^{\prime}\right), \quad \Sigma=J^{2} \mathcal{G}^{p-1} .
$$

In this case, the SD equation in the conformal limit is reparametrization invariant, which means that under an infinitesimal transformation of the time reparametrization $\tau \rightarrow \tau+\epsilon(\tau)$, the two-point function transforms as $\mathcal{G} \rightarrow \mathcal{G}+\delta_{\epsilon} \mathcal{G}$ with 
$\delta_{\epsilon} \mathcal{G}=\left[\Delta\left(\partial_{\tau} \epsilon(\tau)+\partial_{\tau^{\prime}} \epsilon\left(\tau^{\prime}\right)\right)+\epsilon(\tau) \partial_{\tau}+\epsilon\left(\tau^{\prime}\right) \partial_{\tau^{\prime}}\right] \mathcal{G}$,

where $\epsilon(\tau)=\delta \tau$ is an infinitesimal conformal transformation.

In this case, $\mathcal{G}+\delta_{\epsilon} \mathcal{G}$ still solve the conformal SD equations in Eq. (A6).

As a physical consequence, an extra general reparametrization symmetry with an arbitrary function $f(t)$ and conformal invariance is emergent in the IR limit as long as $\omega \ll J$ or equivalently, $J \rightarrow \infty$, namely, in strong interactions, as $\tau \rightarrow f(\tau)$,

$$
\begin{aligned}
& \mathcal{G} \rightarrow\left|f^{\prime}(\tau) f^{\prime}\left(\tau^{\prime}\right)\right|^{\Delta} \mathcal{G}\left(f(\tau), f\left(\tau^{\prime}\right)\right), \\
& \Sigma \rightarrow\left|f^{\prime}(\tau) f^{\prime}\left(\tau^{\prime}\right)\right|^{\Delta(p-1)} \Sigma\left(f(\tau), f\left(\tau^{\prime}\right)\right),
\end{aligned}
$$

where $\Delta=1 / p$ is the conformal dimension of $\mathrm{CFT}_{1}$, which is explicitly broken by the kinetic term $\partial_{\tau}$ in medium energy range, i.e., $\omega \sim J$, which is the explicit symmetry breaking parameter. To be brief, both the two-point function $\mathcal{G}$ and self-energy $\Sigma$ are conformal invariant in the IR background. Therefore, $\beta J \gg 1$ can also be viewed as the conformal limit of the model.

In the IR limit, the action is reparametrization invariant by dropping the kinetic term $\partial_{\tau}$ inside the action, while the solution $\mathcal{G}$ is only $\operatorname{SL}(2, R)$ invariant. Thus, one can view reparametrization invariance as an emergent symmetry of the IR theory, which is spontaneously broken by the conformal solution $\mathcal{G}$. The emergent full reparametrization symmetry, i.e., the Virasoro group SL(2), is presented by the generators

$$
\begin{aligned}
D & =-\tau \partial_{\tau}-\Delta, \quad P=\partial_{\tau}, \quad K=\tau^{2} \partial_{\tau}+2 \tau \Delta, \\
{[D, P] } & =P, \quad[D, K]=-K, \quad[P, K]=-2 D,
\end{aligned}
$$

where $H, D$ and $K$ is a generator of time translation, dilatation and special conformal transformation, respectively. The zero modes in the effective action can be viewed as Nambu-Goldstone (NG) modes for the spontaneous breaking of the full SL(2) conformal symmetry down to $\mathrm{SL}(2, R)$. Since the action is $\operatorname{SL}(2, R)$ gauge invariant, in the path integral, one need to dived the integral by a volume of $\operatorname{SL}(2, R)$.

At zero and finite temperature, the two-point function has a conformal ansatz form at zero and at finite temperature, respectively, as

$$
\begin{aligned}
& T=0: \mathcal{G}(\tau)=\frac{b}{|\tau|^{2 \Delta}} \operatorname{sgn}(\tau), \\
& T \neq 0: \mathcal{G}(\tau)=b\left(\frac{\pi}{\beta \sin \frac{\pi \tau}{\beta}}\right)^{2 \Delta} \operatorname{sgn}(\tau),
\end{aligned}
$$

where $\Delta$ is the IR conformal dimension, which turns out to be inversely proportional to the d.o.f $p$ of the disordered interaction, i.e., $\Delta=1 / p$ and $b^{p}=\left(2 \pi J^{2}\right)^{-1}(1-2 \Delta)$ $\tan (\pi \Delta)$ at leading order in $1 / N$. The Green's function represent the low frequency behavior of the retarded Green's function for the SYK model in the strong coupling limit. The ansatz form above can be obtained by applying the reparametrization at saddle point $f(\tau)=\tau$ in zero temperature case, while in finite temperature case, the time direction is considered Euclidean and compactified into a thermal circle $f(t)=e^{2 \pi i t / \beta}$ or $f(\tau)=\tan (\pi \tau / \beta)$ satisfying $f(\tau+\beta)=f(\tau)$. To be brief, the thermal quantum mechanics or quantum statistics can be achieved through the reparametrization of a zero temperature quantum mechanics, by mapping a straight line of imaginary time $\tau$ to a thermal circle, i.e., $\bar{\tau}=\tan (\pi \tau / \beta)$ with periodic boundary conditions over a periodic lattice length $\beta$.

\section{b. Effective Schwarzian action}

In the low-energy limit, the model can be described by a local effective action proportional to the Schwarzian derivative [1] in terms of Schwarzian theory [23], which can be understood as the dynamics of a Goldstone bosons $f(\tau)$, a near-zero mode for the breaking of reprarametrization invariance [5], with a coefficient of order $(\beta J)^{-1}$ as [1]

$$
S_{\mathrm{eff}}=-\frac{N \alpha}{J} \int d \tau \operatorname{Sch}(f(\tau), \tau),
$$

where $\operatorname{Sch}(f, \tau)$ is the Schwarzian derivative in Eq. (2.2), which is invariant under SL(2) symmetry $f \rightarrow$ $(a f+b) /(c f+d)$ and it is an exact symmetry at zero temperature since $f(\tau)=\tau$. The prime indicates the derivative with respect to the $\tau . f(\tau)$ is the NambuGoldstone bosons, or the zero modes involving large diffeomorphisms, which are nontrivial on the boundary. When one move away from the IR fixed point $(\omega \ll J \rightarrow \infty)$, the NG bosons cease to be zero mode and leads to a nonzero action, i.e., $\operatorname{Sch}(f(\tau), \tau) \neq 0$, e.g., $f(\tau)=\tan (\pi \tau / \beta)$, a black hole with finite temperature as a deformed parameter from $\mathrm{AdS}_{2}$. At the finite temperature, the effective action becomes $S_{\text {eff }}=-2 \pi^{2} N \alpha /(J \beta)$.

The form of the action itself implies an $\operatorname{SL}(2, R)$ invariant solution $f \rightarrow(a f+b) /(c f+d)$ with $a, b, c, d \in$ $\mathbb{R}$ and $a d-b c=1$, which is the same as $\operatorname{SL}(2, C)$. For instance, at finite temperature, $f(\tau)=\tan (\pi \tau / \beta)$, the Schwarzian is $\operatorname{Sch}(f, \tau)=2 \pi^{2} / \beta^{2}$. Since the effect coupling of the theory $1 / g^{2} \propto N \alpha /\left(J \beta^{2}\right)$, at large $N$ and fixed temperature, the theory is weakly coupled, dominated by fluctuations around the saddle point $\tau$, but is strongly coupled at ultra low temperature, i.e., $g \propto \beta$. It also interesting to consider a reparametrization $f(\tau) \rightarrow \tan (\pi \epsilon(\tau) / \beta)$, the Schwarzian becomes $\operatorname{Sch}(f(\tau), \tau)=\operatorname{Sch}(f(\tau), \tau)+$ $2(\pi / \beta)^{2} f^{\prime 2}$. Considering a small reparametrization $\tau \rightarrow \tau+$ $\epsilon(\tau)$, by using the equivalent form of the Schwarzian with terms of the total derivatives, the action can be re-epxressed as a local one as 


$$
\begin{aligned}
S_{\text {eff }} & =\frac{N \alpha}{2 J} \int d \tau\left(\frac{\epsilon^{\prime \prime 2}}{\epsilon^{\prime 2}}-\left(\frac{2 \pi}{\beta}\right)^{2} \epsilon^{\prime 2}\right), \\
\operatorname{Sch}(\epsilon(\tau), \tau) & =\left(\frac{\epsilon^{\prime \prime}}{\epsilon^{\prime}}\right)^{\prime}-\frac{1}{2} \frac{\epsilon^{\prime \prime 2}}{\epsilon^{\prime 2}}
\end{aligned}
$$

where we have dropped the total derivative terms $\left(\epsilon^{\prime \prime} / \epsilon\right)^{\prime}$, the action has an expression of lowest order in derivatives that vanishes for global SL(2) transformation. Consider a small fluctuation on the fixed parametrization $\epsilon(\tau)=\tau+$ $\hat{\epsilon}(\tau)$, and expanded up to quadratic order, one obtains the quantum action in terms of Pseudo Nambu-Goldsonte (PNG) boson field $\hat{\epsilon}$. In this case, not only the SL(2) symmetry is broken by the $\operatorname{SL}(2, R)$-invariant $\operatorname{IR~} G$ solution, but also is explicitly broken, which gives a small Schwarzian action for $\hat{\epsilon}(\tau)$, which is vanishing in the strong interacting limit $J \rightarrow \infty$ at order of large $N$. The effective action is a potential term for the zero mode, thus, the Schwarzian action can be viewed as a mass term for PNG boson. Therefore, the low-energy effective Schwarzian action above makes reparametrization modes $\hat{\epsilon}$ PNG bosons, in terms of soft modes $[9,33]$.

\section{Self-energy}

With the two-point correlation functions $\mathcal{G}(\tau)$, it is also possible to consider the scattering rate of quantum liquid with soft modes from Schwarzian effective action, by doing fourier transformation upon the imaginary time self-energy. In $\mathrm{SYK}_{p}$ model, according to the SD equations in Eq. (A3) or Eq. (A6) with $p=1 / \Delta$, the conformal dimension of the self-energy can be estimated as

$$
\begin{aligned}
& \Sigma_{\Delta} \propto \mathcal{G}(\tau)^{p-1}=\frac{1}{i^{2(\Delta-1)}} \frac{1}{\left[2 \frac{\beta}{2 \pi} \sinh \frac{t}{2} \frac{2 \pi}{\beta}\right]^{2(\Delta-1)}} \\
& \stackrel{\beta \rightarrow \infty}{=} \frac{1}{i^{2(\Delta-1)}} \frac{1}{t^{2(\Delta-1)}},
\end{aligned}
$$

where we have used Eq. (3.30). Thus, $\Sigma_{\Delta}(\omega) \propto \mathcal{G}_{\Delta-1}(\omega) \sim$ $\mathcal{G}_{\Delta}{ }^{\prime \prime}(\omega)$, where the prime denotes the derivative with respect to the frequency $\omega$.

\section{Partition functions}

According to Eq. (A4), by using the thermal parametrization $f(t)=\tan (\pi t / 2)$, the free energy in low temperature expansion i.e., $\beta \gg 1$, can be obtained from the effective action.

From the effective action of the gravity sector in Eq. (2.1), one obtains the free energy

$$
\begin{aligned}
F_{0} & =-\frac{1}{\beta} \ln Z=\frac{1}{\beta} S_{\text {eff }}=\frac{1}{\beta} C_{g} \phi_{r} \int d t \operatorname{Sch}(f(t), t) \\
& =C_{g} \phi_{r} \int d t \frac{1}{2 \beta} \rightarrow C \frac{2 \pi}{\beta} 2 \pi \frac{1}{2 \beta}=C \frac{2 \pi^{2}}{\beta^{2}},
\end{aligned}
$$

which leads to the zero temperature entropy $S=N s_{0}=$ $\beta F=2 \pi^{2} \beta^{-1}$ and the specific heat $C_{V}=N c_{v}=\gamma \beta^{-1}=$ $4 \pi^{2} C \beta^{-1}$, which are both linear in temperature.

While from the effective action of the scalar matter given in Eq. (2.22),

$$
\begin{aligned}
F_{\chi} & =-\frac{1}{\beta} \ln Z_{\chi}=\frac{1}{\beta} \bar{S}_{\mathrm{eff}} \propto \int_{\epsilon}^{\beta-\epsilon} d t\left(\frac{\pi}{\beta \sin \frac{\pi t}{\beta}}\right)^{2 \Delta} \\
& =\frac{\pi^{2 \Delta+\frac{1}{2}} \beta^{1-2 \Delta} \sec (\pi \Delta)}{\Gamma(1-\Delta) \Gamma\left(\Delta+\frac{1}{2}\right)}+\frac{2 \epsilon^{1-2 \Delta}}{2 \Delta-1},
\end{aligned}
$$

where the first term is a finite one as leading IR correction under the case that $\Delta<3 / 2$, since this free energy due to matter $F_{\chi} \propto \beta^{1-2 \Delta}$ dominates over the free energy due to the gravity $F \propto \beta^{-2}$ at low temperature limit. While the second term is a UV divergent term, since it is a constant, thus contributes to the ground state energy density $e_{0}$ as obvious in Eq. (A4).

One can also include the one-loop exact Schwarzian partition function by direct functional path integration of the Schwarzian theory as [23]

$$
Z_{\text {Sch }}=\frac{1}{4 \pi g^{3}} \exp \left(\frac{\pi}{g^{2}}\right), \quad g \equiv \frac{\epsilon^{2}}{C},
$$

from which, the one loop corrections to the free energy is obtained from

$$
F_{\mathrm{Sch}}=-\frac{1}{\beta} \ln Z_{\mathrm{Sch}} \sim-\frac{3}{2} \ln \frac{\beta}{C} .
$$

Therefore, the loop corrections of graviton soft mode to the scalar matter field turns to contributes a finite logarithmic temperature term for the free energy.

\section{APPENDIX B: WAVE FUNCTION IN GLOBAL AdS $_{2}$}

In the main text, we mainly focus on the bosonic retarded Green's function of Schwarzian liquid, a similar procedure might be imposed to fermion's case, which leads to NFL underlying fundamental Dirac or Weyl fermions $[19,35,55,56]$. For $\Delta=1 / 4$ case, one just recovers the FFL of lattice Anderson model [43]. To obtain thermal fermionic retarded Green's function of quantum liquid, one needs to solve the wave functions of Dirac fermions in $(1+1)$-dimensional spacetime in global $\mathrm{AdS}_{2}$ coordinate.

\section{Global $\mathrm{AdS}_{\mathbf{2}}$ spacetime}

In $(1+1)$-dimensional spacetime, the spacetime metric of hyperbolic $\mathrm{AdS}_{2}$ black hole in global coordinate is given by 


$$
d s^{2}=-\left(\frac{r^{2}}{r_{0}^{2}}-1\right) d t^{2}+\left(\frac{r^{2}}{r_{0}^{2}}-1\right)^{-1} d r^{2}
$$

where the second expression is for global coordinate. The gauge field in two-dimensional spacetime is

$$
A_{t}(r)=\mu\left(r-r_{0}\right)
$$

which leads to a constant $U(1)$ field strength, since $F_{t r}=E=-\mu$. The temperature of the black hole turns out to be

$$
T=\frac{1}{2 \pi r_{0}} \text {. }
$$

In Cartesian gauge, the metric

$$
d s^{2}=-d x^{2}-d y^{2}+d z^{2}
$$

can be classified into three classes, according to the index $k=-1$ for embedding conic curves $-x^{2}-y^{2}+z^{2}=$ $k=-1$, which is obvious in the Lorentzian gauge as

$$
\begin{aligned}
d s^{2} & =-\cosh ^{2} \rho d \tau^{2}+d \rho^{2}, \quad \rho \in(0,+\infty), \quad \tau \in(0,2 \pi), \\
(x, y, z) & =\cosh \rho(\sin \tau, \cos \tau, \tanh \rho), \quad y \pm i x=e^{ \pm i \tau} \cosh \rho \\
d s^{2} & =-e^{2 \sigma} d t^{2}+d \sigma^{2}, \quad \sigma \in(-\infty,+\infty), \quad t \in(-\infty,+\infty), \\
(x, y, z) & =\left(e^{\sigma} t, \cosh \sigma-e^{\sigma} t^{2} / 2, \sinh \sigma+e^{\sigma} t^{2} / 2\right), \\
d s^{2} & =-\sinh ^{2} \rho d \tau^{2}+d \rho^{2}, \quad \rho \in(0,+\infty), \quad \tau \in(0,2 \pi), \\
(x, y, z) & =\sinh \rho(\sinh \tau, \cosh \tau, \operatorname{coth} \rho), \\
x \pm z & = \pm e^{ \pm \tau} \sinh \rho,
\end{aligned}
$$

where for hyperbolic case, the $(\rho, \tau)$ is just the usual bulk Rindler like coordinates at positive side of Rindler space with boundary at $\rho \rightarrow \infty$. The Poincaré time $t$ runs from $-\infty$ to $+\infty$, while the Rindler time $\tau$ is $2 \pi$ periodic. In the Schwarzschild gauge,

$$
\begin{aligned}
d s^{2} & =-\left(\frac{r^{2}}{r_{0}^{2}}+1\right) d t^{2}+\left(\frac{r^{2}}{r_{0}^{2}}+1\right)^{-1} d r^{2}=\frac{-d t^{2}+d z^{2}}{\left[\sin \left(z / r_{0}\right)\right]^{2}}, \\
A_{t} & =\mu r_{0}\left(\cot \frac{z}{r_{0}}-1\right), \quad r=r_{0} \cot \frac{z}{r_{0}}, \\
d s^{2} & =-r^{2} d t^{2}+\frac{1}{r^{2}} d r^{2}=\frac{-d t^{2}+d z^{2}}{z^{2}}, \\
A_{t} & =\mu\left(\frac{1}{z}-\frac{1}{z_{0}}\right), \quad r=\frac{1}{z}, \\
d s^{2} & =-\left(\frac{r^{2}}{r_{0}^{2}}-1\right) d t^{2}+\left(\frac{r^{2}}{r_{0}^{2}}-1\right)^{-1} d r^{2}=\frac{-d t^{2}+d z^{2}}{\left[\sinh \left(z / r_{0}\right)\right]^{2}}, \\
A_{t} & =\mu r_{0}\left(\operatorname{coth} \frac{z}{r_{0}}-1\right), \quad r=r_{0} \operatorname{coth} \frac{z}{r_{0}}, \quad(\mathrm{~B} 5)
\end{aligned}
$$

where $r \in\left(r_{0}, \infty\right)$ and $r_{0}$ is the Rindler horizon with $\ell$ being $\mathrm{AdS}_{2}$ radius, the transformation between $r$ and $z$ relates the parabolic orbits and to elliptic/hyperbolic orbits. At infinite boundary, the elliptic and hyperbolic type just reduces to be parabolic one.

\section{Boson in global $\mathbf{A d S}_{2}$}

Consider the $\mathrm{AdS}_{2}$ metric in global coordinates as

$$
d s^{2}=\ell^{2}\left(d \rho^{2}-\cosh ^{2} \rho d \tau^{2}\right), \quad \ell^{2} \equiv-\frac{1}{4 c_{\mu}},
$$

where $\rho \in(-\infty,+\infty)$ and $\tau \in(-\infty,+\infty), c_{\mu}$ contains UV information of two-dimensional gravity.

The global coordinates are within the range $\rho \in(\infty, 0)$ for $z \in(0, \infty)$. Thus, it is expected that the theory is dual to copies of conformal quantum mechanics $\mathrm{CQM}_{1}$ and $\mathrm{CQM}_{2}$ on two boundaries via $\mathrm{AdS}_{2} / \mathrm{CFT}_{1}$. The Klein-Gordon equation in this coordinates becomes

$$
\phi^{\prime \prime}+\tanh \rho \phi^{\prime}+\left(\omega^{2} \operatorname{sech}^{2} \rho-\frac{m^{2}}{4 c_{\mu}}\right) \phi=0
$$

which gives the wave functions as

$$
\rho(z)=\left(z^{2}-1\right)^{1 / 4}\left[c_{1} P_{i \omega-\frac{1}{2}}^{\nu_{1}}(z)+c_{2} Q_{i \omega-\frac{1}{2}}^{\nu_{1}}(z)\right],
$$

where $z \equiv \tanh \rho$ and

$$
\nu_{1}=\sqrt{\frac{1}{4}-\frac{m^{2}}{4 c_{\mu}}} .
$$

\section{Fermion in global $\mathbf{A d S}_{2}$}

The Dirac equation in $(1+1)$-dimensional spacetime can be expressed as

$$
\left(\Gamma^{\mu} D_{\mu}-m\right) \psi=0
$$

where $\psi=\left(\psi_{-}, \psi_{+}\right)^{T}$ is a two component spinor, $\Gamma^{\mu}=$ $e_{a}^{\mu} \gamma^{a}$ where $e_{a}^{\mu}$ is the inverse of the vielbein $e_{\mu}^{a}$, which relates the metric in curved spacetime frame to the local flat Minkowski frame

$$
g_{\mu \nu}=\eta_{a b} e_{\mu}^{a} e_{\nu}^{b},
$$

where $\eta^{a b}=\operatorname{diag}(-1,+1)$ is Lorentzian metric for local inertial frame.

The covariant derivatives are $D_{\mu}=\partial_{\mu}+\Omega_{\mu}$ where $\Omega_{\mu} \equiv \omega_{\mu}^{a b} \gamma_{a b} / 4$ and $\omega_{\mu}^{a b}$ is the spin connection $\omega_{\mu}^{a b}=$ $e_{\nu}^{a}\left(\partial_{\mu} e^{b \nu}+e^{b \lambda} \Gamma_{\lambda \mu}^{\nu}\right)$, where $\Gamma_{\lambda \mu}^{\nu}$ is the Christoffel symbols and $\gamma^{a b}=\left[\gamma^{a}, \gamma^{b}\right] / 2$ is the generator of Lorentz rotations. $\gamma^{a}$ is the gamma matrices of Dirac fermions, which satisfy the anticommutation relations 


$$
\left\{\gamma^{a}, \gamma^{b}\right\}=2 \eta^{a b}
$$

where $\eta^{a b}=\operatorname{diag}(-1,+1)$ is two-dimensional Lorentzian metric for local inertial frame.

In two dimensions, one may choose $\gamma^{a}$ as a real representation [56]

$$
\begin{aligned}
& \gamma^{0}=\gamma^{\underline{t}}=i \sigma^{2}=\left(\begin{array}{cc}
0 & 1 \\
-1 & 0
\end{array}\right), \\
& \gamma^{1}=\gamma^{\underline{x}}=\sigma^{1}=\left(\begin{array}{ll}
0 & 1 \\
1 & 0
\end{array}\right),
\end{aligned}
$$

where $\sigma^{a}$ are Pauli matrix. In the representation, the chirality operator is

$$
\gamma^{3}=\gamma^{0} \gamma^{1}=\sigma^{3}
$$

In the conformal gauge, or light cone gauge, one has

$$
\begin{aligned}
& \gamma_{+} \equiv \frac{1}{2}\left(\gamma^{0}+\gamma^{1}\right)=\left(\begin{array}{ll}
0 & 1 \\
0 & 0
\end{array}\right), \\
& \gamma_{-} \equiv \frac{1}{2}\left(\gamma^{0}-\gamma^{1}\right)=\left(\begin{array}{ll}
0 & 0 \\
1 & 0
\end{array}\right) .
\end{aligned}
$$

Thus, the wave function contains two components carrying opposite chirality as $\phi=\left(\psi_{-}, \psi_{+}\right)^{T}$.

The vielbein $e_{\mu}^{a}$ allows the $\gamma$ matrices to satisfy a generalized algebra in the curved spacetime as

$$
\left\{\gamma^{\mu}, \gamma^{\nu}\right\}=2 g^{\mu \nu},
$$

where $\gamma^{\mu} \equiv \gamma^{a} e_{a}^{\mu}$. In $(1+1)$-dimensional spacetime, the gamma matrix can simplifies the Dirac equations in curved spacetime as

$$
\left[\gamma^{a}\left(e_{a}^{\mu} \partial_{\mu}+\frac{1}{2} \frac{1}{\sqrt{-g}} \partial_{\mu}\left(\sqrt{-g} e_{a}^{\mu}\right)\right)-m\right] \psi=0 .
$$

By using a coordinate transformation

$$
\sinh \rho \equiv \tan \sigma, \quad \sigma \in(-\pi / 2, \pi / 2)
$$

which transforms the boundary of the spacetime at $\rho=$ $\pm \infty$ into $\sigma= \pm \pi / 2$, the global $\mathrm{AdS}_{2}$ metric in Eq. (B6) becomes equivalently as

$$
d s^{2}=\ell^{2} \frac{-d \tau^{2}+d \sigma^{2}}{\cos ^{2} \sigma} .
$$

The vielbein and nonvanishing spin connection in the global $\mathrm{AdS}_{2}$ coordinate in Eq. (B6) are given by $e_{\tau}^{\frac{\tau}{\tau}}=\ell \cosh \rho, \quad e_{\rho}^{\frac{\rho}{\rho}}=\ell^{2}, \quad \omega_{\tau}^{\underline{\underline{\tau}} \underline{\underline{\tau}}}=-\omega_{\bar{\tau}}^{\frac{\rho}{\underline{\tau}}}=\sinh \rho$.

In the momentum space by assuming the time translation invariance, i.e., $\psi_{\mp}(t, \rho) \rightarrow e^{-i \omega t} \psi_{\mp}(\omega, \rho)$. The Dirac equation $(\not D-m) \psi=0$ in the global $\mathrm{AdS}_{2}$ coordinate in Eq. (B6), becomes

$$
\begin{aligned}
& \left(\partial_{\rho}-\frac{i \omega}{\cosh \rho}-\frac{1}{2} \tanh \rho\right) \psi_{+}-m \ell \psi_{-}=0 \\
& \left(\partial_{\rho}+\frac{i \omega}{\cosh \rho}-\frac{1}{2} \tanh \rho\right) \psi_{-}-m \ell \psi_{+}=0
\end{aligned}
$$

which can be combined to be two decoupled equations of motion for $\psi_{\mp}$, respectively, as

$$
\begin{aligned}
& \left(\partial_{\rho}^{2}+\tanh \rho \partial_{\rho}+\frac{1}{4}+m^{2} \ell^{2} \mp i \omega \frac{\tanh \rho}{\cosh \rho}+\frac{\omega^{2}+1 / 4}{\cosh ^{2} \rho}\right) \\
& \quad \times \psi_{\mp}=0 .
\end{aligned}
$$

Alternatively, the Dirac equation in the global $\mathrm{AdS}_{2}$ coordinate in Eq. (B19) is

$$
\begin{aligned}
& \left(\partial_{\sigma}-i \omega-\frac{\tan \sigma}{2}\right) \psi_{+}-\frac{m \ell}{\cos \sigma} \psi_{-}=0, \\
& \left(\partial_{\sigma}+i \omega-\frac{\tan \sigma}{2}\right) \psi_{-}-\frac{m \ell}{\cos \sigma} \psi_{+}=0,
\end{aligned}
$$

which can be combined to be two decoupled equations of motion for $\psi_{\mp}$, respectively, as

$$
\left(\partial_{\sigma}^{2} \mp i \omega \tan \sigma+\frac{1 / 4+m^{2} \ell^{2}}{\cos ^{2} \sigma}+\omega^{2}+\frac{1}{4}\right) \psi_{\mp}=0 .
$$

The solutions to the Dirac equation in Eq. (B22) or Eq. (B24) turn out to be

$$
\begin{aligned}
\psi_{-}= & (z+1)^{-i m \ell}\left[c_{2} z^{-\omega / 2}{ }_{2} F_{1}(-i m \ell,-i m \ell-\omega\right. \\
& \left.+\frac{1}{2} ; \frac{1}{2}-\omega ;-z\right)+c_{1} \sqrt{z} z^{\omega / 2}{ }_{2} F_{1}(1-i m \ell,-i m \ell \\
& \left.\left.+\omega+\frac{1}{2} ; \omega+\frac{3}{2} ;-z\right)\right], \\
\psi_{+}= & (z+1)^{-i m \ell}\left[c_{4} \sqrt{z} z^{-\omega / 2}{ }_{2} F_{1}(1-i m \ell,-i m \ell-\omega\right. \\
& \left.+\frac{1}{2} ; \frac{3}{2}-\omega ;-z\right)+c_{3} z^{\omega / 2}{ }_{2} F_{1}(-i m \ell,-i m \ell+\omega \\
& \left.\left.+\frac{1}{2} ; \omega+\frac{1}{2} ;-z\right)\right],
\end{aligned}
$$

where $\psi_{\mp}=\psi_{\mp}(z)$ with $z=e^{2 i \sigma}$. 
APPENDIX C: HIGHER-DIMENSIONAL GENERALIZATION

\section{Boson in $d+1$-dimensional spacetime}

a. Klein-Gordon bulk equation of motion

The equation of motion in coordinate space for $\phi$ with action

$S=-\int d^{d+1} x \sqrt{-g}\left[g^{M N}\left(D_{M} \phi\right)^{\star} D_{N} \phi+m^{2} \phi^{\star} \phi\right]$,

where $D_{M} \equiv \nabla_{N}-i q A_{N}$. From the action, one can obtain the equations of motion for the complex scalar,

$$
g^{M N}\left(\nabla_{M}-i q A_{M}\right)\left(\nabla_{N}-i q A_{N}\right) \phi-m^{2} \phi=0 .
$$

Assuming the spacetime metric is

$$
d s^{2}=-g_{t t}(r) d t^{2}+g_{r r}(r) d r^{2}+g_{x x}(r) d x^{2} .
$$

Then, the EOM of the charged scalar is

$$
\begin{gathered}
\frac{1}{g_{r r}}\left[\partial_{r}^{2}+\frac{1}{2}\left(\frac{g_{t t}^{\prime}}{g_{t t}}-\frac{g_{r r}^{\prime}}{g_{r r}}+(d-1) \frac{g_{x x}^{\prime}}{g_{x x}}\right) \partial_{r}\right] \phi \\
\quad+\left(\frac{1}{g_{x x}} \partial_{x}^{2}+\frac{1}{g_{t t}}\left(\omega+q A_{t}\right)^{2}-m^{2}\right) \phi=0,
\end{gathered}
$$

where $\phi=\phi(t, r, x)$, and for the briefness, we have dropped $r$ dependence of $g_{t t}, g_{r r}, g_{x x}$. The EOM of the charged scalar can reexpressed as

$$
\left[\frac{\partial_{r}\left(\sqrt{-g} g^{r r} \partial_{r}\right)}{\sqrt{-g}}+\left(\frac{\partial_{x}^{2}}{g_{x x}}+\frac{\left(\omega+q A_{t}\right)^{2}}{g_{t t}}\right)-m^{2}\right] \phi=0 .
$$

where we have used the relation

$$
\frac{1}{A(r)} \partial_{r}\left[A(r) \partial_{r}\right]=\partial_{r}^{2}+\ln A(r)^{\prime} \partial_{r}
$$

so that $A(r) \equiv \sqrt{-g} g^{r r}=\sqrt{g_{t t} g^{r r} g_{x x}^{d-1}}$. By doing Fourier transformation as,

$$
\phi\left(r, x^{\mu}\right)=\int \frac{d^{d} k}{(2 \pi)^{d}} e^{i k_{\mu} x^{\mu}} \phi\left(r, k_{\mu}\right),
$$

where $k_{\mu}=(-\omega, \vec{k})$ and $x^{\mu}=\left(t, x^{i}\right)$. The equation of motion for $\phi=\phi\left(r, k_{\mu}\right)$ is given by

$-\frac{1}{\sqrt{-g}} \partial_{r}\left(\sqrt{-g} g^{r r} \partial_{r} \phi\right)+\left[g^{i i}\left(\vec{k}^{2}-u^{2}\right)+m^{2}\right] \phi=0$, where

$$
u=\sqrt{\frac{g_{x x}}{g_{t t}}}\left[\omega+q A_{t}(r)\right] .
$$

\section{b. Charged scalar in $\mathbf{R N}-\mathbf{A d S}_{d+1}$ spacetime}

In the $\operatorname{AdS}_{d+1}$ spacetime in the energy coordinate $z$,

$$
d s^{2}=\frac{\ell^{2}}{z^{2}}\left(-f(z) d t^{2}+\frac{d z^{2}}{f(z)}+d x_{d-1}^{2}\right),
$$

where the redshift factor and the gauge fields are

$$
\begin{aligned}
f(z) & =1+\frac{d}{d-2}\left(\frac{z}{z_{\star}}\right)^{2(d-1)}-\frac{2(d-1)}{d-2}\left(\frac{z}{z_{\star}}\right)^{d}, \\
A_{t} & =\mu\left(1-\frac{z^{d-2}}{z_{\star}^{d-2}}\right) .
\end{aligned}
$$

The EOM for the charged scalar becomes

$$
\begin{gathered}
{\left[f(z) \partial_{z}^{2}+\left(f^{\prime}(z)-(d-1) \frac{f(z)}{z}\right) \partial_{z}\right.} \\
\left.-\frac{\left(\partial_{t}-i q A_{t}\right)^{2}}{f(z)}+\partial_{x}^{2}-\frac{m^{2} \ell^{2}}{z^{2}}\right] \phi=0,
\end{gathered}
$$

where $\phi=\phi(t, z, x)$ and

$$
u(z)=\frac{1}{\sqrt{f(z)}}\left[\omega+q \mu\left(1-\frac{z^{d-2}}{z_{\star}^{d-2}}\right)\right] .
$$

Note that we have chosen the gauge so that the scalar potential is zero at the horizon $\left(r=r_{0}, z=z_{0}\right)$, and as a result.

$$
A_{t}(z) \rightarrow \mu, \quad u(z) \rightarrow \omega+q \mu . \quad(z \rightarrow 0) .
$$

This implies that $\omega$ should correspond to the difference of the boundary theory frequency from $q \mu$, thus the low energy limit really means very close to the effective chemical potential $q \mu$. In the momentum space $\left(\partial_{t} \rightarrow i \omega\right.$ and $\left.\partial_{x} \rightarrow-i k\right)$, the EOM can be reexpressed as

$$
\left[z^{d-1} \partial_{z}\left(\frac{f(z)}{z^{d-1}} \partial_{z}\right)+\frac{\left(\omega-q A_{t}\right)^{2}}{f(z)}-k^{2}-\frac{m^{2} \ell^{2}}{z^{2}}\right] \phi=0,
$$

where $\phi=\phi(\omega, z)$. We will most interested in the case $T=0$, where $f(z)$ is shown in Eq. (C11).

\section{c. CFT $_{1}$ correlation functions}

Consider a massive neutral scalar field $\phi$ with mass in the bulk action 


$$
S_{\phi}=-\frac{1}{2} \int d^{2} x \sqrt{-g}\left[(\nabla \phi)^{2}+m^{2} \phi^{2}+\cdots\right],
$$

where $\cdots$ denotes the interactions terms or highdimensional operator, which is irrelevant at the moment. Consider a pure anti-de Sitter spacetime with a $\mathrm{AdS}_{2}$ metric in conformal coordinate

$$
d s^{2}=\frac{\ell^{2}}{z^{2}}\left(-d t^{2}+d z^{2}\right),
$$

where $\mu=(t)$, the signature of the ordinary spacetime is chosen as $(-1,1)$, and $z$ is the coordinate of extra dimension where the gravity is penetrating. In the coordinates, the $\mathrm{AdS}_{2}$ boundary is lying at $z=0$.

The equations of motion is $\nabla^{2} \phi-m^{2} \phi=0$ in the $\mathrm{AdS}_{2}$ metric is

$$
\partial_{z}^{2} \phi-\frac{1}{z} \partial_{z} \phi-\partial_{t}^{2} \phi-\frac{m^{2} \ell^{2}}{z^{2}} \phi=0
$$

where $\phi=\phi(z, t)$. In the infinite boundary $z \rightarrow 0$, the scalar wave equations is dominated by pole at $z=0$,

$$
\partial_{z}^{2} \phi-\frac{1}{z} \partial_{z} \phi-\frac{m^{2} \ell^{2}}{z^{2}} \phi=0 .
$$

In the infinite boundary, the asymptotic solution to the scalar wave function has the expansion,

$$
\phi(t, z) \sim A(\omega) z^{\Delta_{-}}+B(\omega) z^{\Delta_{+}}, \quad z \rightarrow 0,
$$

where the two exponents are, respectively,

$$
\Delta_{ \pm}=\frac{1}{2} \pm \nu_{1}, \quad \nu_{1}=\sqrt{\frac{1}{4}+m^{2} \ell^{2}} .
$$

The conformal dimension $\Delta_{ \pm}$are two roots of the quadratic equations

$$
\Delta(\Delta-1)=m^{2} \ell,
$$

which are consistent with the results from AdS2 gravity [71].

Let's consider an operator $\mathcal{O}$ of the boundary field theory, dual to a bulk scalar field $\phi(t, z)$ with mass $m$ and charge $q$. The boundary field theory in the UV, e.g., $\mathrm{CFT}_{1}$ in the UV is characterized only and completely by the dimension of the operator $\mathrm{CFT}_{1}$, e.g., $\Delta$ which is given in term of bulk quantities as shown in Eq. (C21) for neutral bulk field. The boundary field theory in the IR, e.g., $\mathrm{CFT}_{1}$, or $(0+1)$-dimensional CQM [72-74], is characterized by the scaling dimension of the operator $\Phi$. Then we have the following correspondence to the conformal field theory at the boundary.
(1) $\Delta_{+}$is the conformal dimension of the dual operator $\mathcal{O}$;

(2) $A(\omega)$ : the coefficient of the more dominant term $\left(\left|z^{\Delta_{-}}\right| \gg\left|z^{\Delta_{+}}\right|\right.$, when $\left.z \rightarrow 0\right)$ in the infinite boundary condition $z \rightarrow 0$, can be identified as the source for $\mathcal{O}$, which is equivalent to adding to the Lagrangian of the boundary theory a source term $\delta S_{B}=$ $\int d t A(t) \mathcal{O}(t)$.

(3) $B(\omega)$ : the coefficient of the subdominant term in the infinite boundary condition, can be identified as the expectation value of the operator $\mathcal{O}$, e.g., $\langle\mathcal{O}\rangle=$ $2 \nu_{1} B(t)$.

(4) The linear response function in momentum space for $\mathcal{O}$ is $G_{R}(\omega, \vec{k})=2 \nu_{1} B(\omega) / A(\omega)$, where the $B$ and $A$ are the quantities after doing Fourier transform along the boundary directions $z$. The ratio is determined by a regularity condition at the interior of the spacetime, e.g., for the horizon brane, it is the infalling condition at the horizon.

With the above identification, we have the following physical consequence:

(1) $A(\omega)=0 \quad\left(\delta S_{B}=0\right)$ but $B(\omega) \neq 0$ : Spontaneous symmetry breaking (SSB), the operator $\mathcal{O}$ has developed an expectation value without a source, the linear response function is divergent $G_{R}(\omega) \rightarrow \infty$.

(2) $A(\omega) \neq 0 \quad\left(\delta S_{B} \neq 0\right)$ but $B(\omega) \neq 0$ : Symmetry breaking $(\mathrm{SB})$, the operator $\mathcal{O}$ has developed an expectation value with a source, the linear response function is finite.

(3) $A(\omega) \neq 0$ but $B(\omega)=0$ : No expectation value is developed, the linear response function is absent, namely no response at all.

\section{d. CFT $_{d}$ correlation functions}

At the momenta, let's re-visit the asymptotic behavior of the bulk scalar, in the infinite boundary for the $\operatorname{AdS}_{d+1}$ spacetime with $d \geq 3, z \rightarrow 0\left(f(z) \rightarrow 1, A_{t} \rightarrow \mu\right)$ :

$$
\begin{aligned}
& \phi\left(x^{\mu}, z\right) \sim A(x) z^{\Delta_{-}}+B(x) z^{\Delta_{+}}, \quad z \rightarrow 0, \\
& \phi\left(k_{\mu}, r\right) \sim A\left(k_{\mu}\right) r^{-\Delta_{-}}+B\left(k_{\mu}\right) r^{-\Delta_{+}},
\end{aligned}
$$

where the two exponents are, respectively,

$$
\Delta_{ \pm}=\frac{d}{2} \pm \nu_{d}, \quad \nu_{d}=\sqrt{\frac{d^{2}}{4}+m^{2} \ell^{2}},
$$

and it is worthy of noticing that $\Delta_{-}=d-\Delta_{+}$. Thus, the retarded Green function in the infinite boundary condition is

$$
G_{R}(\omega, \vec{k})=K \frac{B\left(k_{\mu}\right)}{A\left(k_{\mu}\right)},
$$

where we just consider the standard quantization in the discussion and $K$ is a positive constant, which is 
independent of $k_{\mu}=(-\omega, \vec{k})$. The asymptotic behavior is obtained by solving the EOM in the $\operatorname{AdS}_{d+1}(d \geq 3)$ metric

$$
\left(\partial_{z}^{2}-\frac{d-1}{z} \partial_{z}-\left[\left(\partial_{t}-i q \mu\right)^{2}-\partial_{x}^{2}\right]-\frac{m^{2} \ell^{2}}{z^{2}}\right) \phi=0,
$$

where $\phi=\phi(t, z, x)$. For neutral massive scalar case $q=0$. The EOMs of the scalar wave equations are dominated by pole at $z=0$, for $d \geq 3$, in the infinite boundary $z \rightarrow 0$, namely,

$$
\left(\partial_{z}^{2}-\frac{d-1}{z} \partial_{z}-\frac{m^{2} \ell^{2}}{z^{2}}\right) \phi(t, z, x)=0 .
$$

Assume that $\phi$ is an in-falling wave at the horizon $\left(\partial_{x} \rightarrow i k, \partial_{t} \rightarrow-i \omega\right)$, then the EOM in boundary in momentum space becomes

$$
\left(\partial_{z}^{2}-\frac{d-1}{z} \partial_{z}+\left[(\omega+q \mu)^{2}-k^{2}\right]-\frac{m^{2} \ell^{2}}{z^{2}}\right) \phi=0,
$$

where $\phi=\phi(\omega, z, k)$. Therefore the boundary theory energy corresponds to $\omega+q \mu$, and $\omega$ should be interpreted as measured from the effective chemical potential $q \mu>0$. The wave functions becomes

$$
\phi(z) \stackrel{z \rightarrow 0}{\sim} z^{d / 2}\left[c_{1} J_{\nu_{d}}(\bar{\omega} z)+c_{2} Y_{\nu_{d}}(\bar{\omega} z)\right]
$$

where $\bar{\omega}=\sqrt{(\omega+q \mu)^{2}-k^{2}}$, and the conformal dimension is $\nu_{d}$ is defined in Eq. (C24).

Take charged scalar in $\mathrm{AdS}_{2}$ vacuum as an example. In this case, $d=1, q=0, k=0$, and the wave functions becomes

$$
\phi(z)=\sqrt{z}\left[c_{1} J_{\nu_{1}}(z \omega)+c_{2} Y_{\nu_{1}}(z \omega)\right],
$$

in the near horizon limit, one obtains the asymptotic behavior of the boson wave function as

$$
\begin{aligned}
\phi(z) \stackrel{z \rightarrow \infty}{\sim} c_{1} \frac{\cos \left[\frac{\pi \nu_{1}}{2}-(\omega+q \mu) z\right]-\sin \left[\frac{\pi \nu_{1}}{2}-(\omega+q \mu) z\right]}{\sqrt{\pi} \sqrt{\omega}} \\
-c_{2} \frac{\sin \left[\frac{\pi \nu_{1}}{2}-(\omega+q \mu) z\right]+\cos \left[\frac{\pi \nu_{1}}{2}-(\omega+q \mu) z\right]}{\sqrt{\pi} \sqrt{\omega}} \\
=\frac{\left(\frac{1}{2}+\frac{i}{2}\right)\left(c_{1}+i c_{2}\right)}{\sqrt{\pi} \sqrt{\mu q+\omega}} e^{-i(\omega+q \mu) z+i \frac{\pi}{2} \nu_{1}} \\
+\frac{\left(\frac{1}{2}-\frac{i}{2}\right)\left(c_{1}-i c_{2}\right)}{\sqrt{\pi} \sqrt{\mu q+\omega}} e^{i(\omega+q \mu) z-i \frac{\pi}{2} \nu_{1}} .
\end{aligned}
$$

The in-falling wave is $e^{-i \omega t+i(\omega+q \mu) z}$ which entails that $c_{1}=$ $-i c_{2}$ so that the outgoing waves are isolated. On the other hand, in the infinite boundary $z \rightarrow 0$, one has

$$
\begin{aligned}
\phi(z) \stackrel{z \rightarrow 0}{\sim} & \frac{2^{-\nu_{1}}\left(c_{2} \cot \left(\pi \nu_{1}\right)+c_{1}\right)(\mu q+\omega)^{\nu_{1}}}{\Gamma\left(\nu_{1}+1\right)} z^{\frac{1}{2}+\nu_{1}} \\
& -\frac{c_{2} 2^{\nu_{1}} \Gamma\left(\nu_{1}\right)(\mu q+\omega)^{-\nu_{1}}}{\pi} z^{\frac{1}{2}-\nu_{1}} \\
= & B(\omega) z^{\nu_{1}}+A(\omega) z^{-\nu_{1}}
\end{aligned}
$$

where $A$ and $B$ are identified as source and response, respectively. The two-point Green's function can be read as

$$
\begin{aligned}
G(\omega) & =\frac{B(\omega)}{A(\omega)}=-\frac{\pi\left[\cot \left(\pi \nu_{1}\right)-i\right]}{4^{\nu_{1}} \Gamma\left(\nu_{1}\right) \Gamma\left(\nu_{1}+1\right)} \omega^{2 \nu_{1}} \\
& =-\frac{\pi 4^{-\nu_{1}} e^{i \pi \nu_{1}}}{\Gamma\left(\nu_{1}\right) \Gamma\left(\nu_{1}+1\right)} \frac{\omega^{2 \nu_{1}}}{\sin \left(\pi \nu_{1}\right)} .
\end{aligned}
$$

\section{AdS $_{\mathbf{2}}$ from RN-AdS $\mathbf{S}_{d+1}$}

The $\mathrm{AdS}_{2}$ spacetime background can be generalized into high-dimensional spacetime, e.g., as an embedded boundary in the near horizon boundary of a charged RN black hole in $\operatorname{AdS}_{d+1}$.

$$
d s^{2}=\left(\frac{r}{\ell}\right)^{2}\left(-f(r) d t^{2}+d x^{2}\right)+\left(\frac{\ell}{r}\right)^{2} \frac{d r^{2}}{f(r)},
$$

where

$$
\begin{aligned}
f(r) & =1+\frac{d}{d-2}\left(\frac{r_{\star}}{r}\right)^{2(d-1)}-\frac{2(d-1)}{d-2}\left(\frac{r_{\star}}{r}\right)^{d}, \\
A_{t} & =\mu\left[1-\left(\frac{r_{\star}}{r}\right)^{d-2}\right] .
\end{aligned}
$$

In the IR limit at large distance, we have $f(r) \approx 1$, or energy scale is much larger than the chemical potential $\mu$ (but still much less than the UV scale) is simply a vacuum with conformal symmetry $\mathrm{AdS}_{d+1}$. The near horizon geometry is given by $\mathrm{AdS}_{2} \times \mathbb{R}^{d-1}$, i.e., $d s^{2}=d s_{\mathrm{AdS}_{2}}^{2}+$ $\left(r_{\star}^{2} / \ell^{2}\right) d x^{2}$, which indicates the boundary system should develop an enhanced symmetry group including scaling invariance. For $T=\frac{1}{2 \pi \zeta_{0}}$ and $T=0$ case, respectively, one has $\mathrm{AdS}_{2}$ in global and local/Poincaré coorindates, respectively, as

$$
\begin{array}{r}
d s_{N \mathrm{AdS}_{2}}^{2}=\frac{\ell_{2}^{2}}{\zeta^{2}}\left[-\left(1-\frac{\zeta^{2}}{\zeta_{0}^{2}}\right) d t^{2}+\left(1-\frac{\zeta^{2}}{\zeta_{0}^{2}}\right)^{-1} d \zeta^{2}\right] \\
=\frac{-d \bar{t}^{2}+d z^{2}}{[\sinh (z / \ell)]^{2}}, \quad A_{t}=-\frac{E \ell^{2}}{\zeta}\left(1-\frac{\zeta}{\zeta_{0}}\right) \\
d s_{\mathrm{AdS}_{2}}^{2}=\frac{\ell_{2}^{2}}{\zeta^{2}}\left(-d t^{2}+d \zeta^{2}\right), \quad A_{t}=\frac{e_{d}}{\zeta},
\end{array}
$$

where $\ell_{2}$ is the curvature radius of $\mathrm{AdS}_{2}$ given by 


$$
\ell_{2}=\frac{1}{d(d-1)} \ell
$$

For the second equality, we have used the coordinate transformation as

$\zeta=\zeta_{0} \tanh \frac{z}{\ell_{2}}, \quad \bar{t}=\frac{\ell_{2}}{\zeta_{0}} t, \quad A_{\bar{t}}=E \ell\left(\operatorname{coth} \frac{z}{\ell_{2}}-1\right)$.

In the finite temperature limit $\zeta \rightarrow \zeta_{0}$, the metric and the vector potential of the Maxwell field are dominated by

$$
d s^{2} \rightarrow \frac{\ell_{2}^{2}}{\zeta^{2}}\left(1-\frac{\zeta^{2}}{\zeta_{0}^{2}}\right)^{-1} d \zeta^{2}+\frac{r_{\star}^{2}}{\ell^{2}} d x^{2}, \quad A_{t}(\zeta) \rightarrow 0 .
$$

The time direction also shrinks to zero, and the spatial direction approaches a constant, the Maxwell field approaches zero. The RG scales is flowing from $\operatorname{AdS}_{d+1}$ with scale $z$ to near horizon boundary with scale $z_{0}$, a single scale of the boundary theory. In the boundary field theory aspect, the theory flows from $\mathrm{CFT}_{d}$ in the UV to the IR-CFT 1 , which is a conformal symmetries of a $(0+1)$-dimensional conformal quantum mechanics (CQM) [72-74], including the scaling symmetry in the time direction. Therefore, the corresponding IR fixed point is just a IR-CFT 1 , which is a conformal symmetry only in the time direction. The new conformal symmetry is emergent and has relation with the collective motion of the large number of charged excitation. Of course, one has to take a notice that the spatial direction can also have important physical consequence.

In the zero temperature limit $\zeta \rightarrow 0$, the metric and the gauge field in Eq. (C36) reduce to the $T=0$ case in Eq. (C37). It is worthy of emphasizing here that the central charge is infinite, since it is proportional to the volume of the $d$-dimensional transverse space $\mathbb{R}^{d-1}$. To have a finite central charge one could replace $R^{d-1}$ by other manifold, i.e., a torus.

The time direction shrinks to zero, and the spatial direction approaches a constant, the Maxwell field approaches zero. The $\mathrm{AdS}_{2}$ symmetry is emergent in the near-horizon region. The $\mathrm{AdS}_{2}$ is isomorphic to a full $\mathrm{SL}(2, R)$ symmetry, which own the scaling isometry:

$$
t \rightarrow \lambda t, \quad \zeta \rightarrow \lambda \zeta, \quad x \rightarrow x
$$

where only the time sector scales. The finite $t$ corresponds to the long-time limit of the original time coordinate, meanwhile the short-distance limit of the original spatial coordinates. Thus, the metric obtained above should apply to the low-frequency limit, since $\omega$ is the frequency conjugate to $t$

$$
\omega \sim T \ll \mu .
$$

In the low-frequency limit, the d-simensional boundary theory at finite charge density should be described by a $\mathrm{CFT}_{1}$, in terms of IR CFT of the boundary theory, which is an emergent conformal symmetry due to collective behavior of a large number of degrees of freedom. It is not related to the microscopic conformal symmetry in the UV, which is broken by finite charge density.

The metric is the $\mathrm{AdS}_{2}$ slice of the high-dimensional $\mathrm{RN}-\mathrm{AdS}_{d+1}$, the letters with a bar above are associated with $\bar{t}, T$, and $\bar{T}$ and are the Hawking temperature with respect to the coordinates $t$ and $\bar{t}$, respectively. In this case, the frequency conjugated to the rescaled time $\bar{t}$ becomes

$$
\bar{\omega}=\frac{\zeta_{0}}{\ell} \omega, \quad \frac{\bar{\omega}}{2 \pi \bar{T}}=\frac{\omega}{2 \pi T}, \quad \bar{T}=\frac{1}{2 \pi \ell_{2}} .
$$

\section{a. Near horizon field equations of motion}

Consider a massive scalar field $\phi$ with mass in the bulk action as

$S=-\int d^{d+1} x \sqrt{-g}\left[g^{M N}\left(D_{M} \phi\right)^{\star}\left(D_{N} \phi\right)+m^{2} \phi^{\star} \phi\right]$,

where the covariant derivative is defined as $D_{N} \equiv \nabla_{N^{-}}$ $i q A_{N}$. From the action, one can obtain the equations of motion for the complex scalar,

$$
\left[g^{M N}\left(\nabla_{M}-i q A_{M}\right)\left(\nabla_{N}-i q A_{N}\right)-m^{2}\right] \phi=0 .
$$

Let's consider a charged scalar field in the background metric and gauge field at zero temperature $T=0$, which is given by Eq. (C37),

$d s^{2}=\frac{\ell_{2}^{2}}{\zeta^{2}}\left(-d t^{2}+d \zeta^{2}\right)+\frac{r_{\star}^{2}}{\ell^{2}} d x^{2}, \quad A_{t}=\frac{e_{d}}{\zeta}$,

which recovers the asymptotic $\mathrm{AdS}_{2}$ spacetime in Eq. (B5), by setting $\ell_{2}=1$ and $e_{d}=\mu$. To be explicit, by considering that $g^{x x}=1 / r_{\star}^{2}, A_{x}=0$, the field equations become

$\left(\partial_{\zeta}^{2}-\partial_{t}^{2}+\frac{\ell^{2} \ell_{2}^{2}}{r_{\star}^{2} \zeta^{2}} \partial_{x}^{2}+\frac{2 i q e_{d}}{\zeta} \partial_{t}+\frac{q^{2} e_{d}^{2}}{\zeta^{2}}-\frac{m^{2} \ell_{2}^{2}}{\zeta^{2}}\right) \phi=0$,

where $\phi=\phi(t, \zeta)$. Alternatively, one can expand $\phi$ in momentum space by doing Fourier transformation,

$$
\phi(t, \vec{x}, \zeta)=\int \frac{d \omega d \vec{k}}{(2 \pi)^{d}} e^{-i \omega t+i \vec{k} \cdot \vec{x}} \phi(\omega, k, \zeta),
$$

one obtains

$$
S=-\int d^{2} x \sqrt{-g}\left[g^{a b}\left(D_{a} \phi\right)_{\vec{k}}^{\star} D_{b} \phi_{\vec{k}}+m_{k}^{2} \phi_{\vec{k}}^{\star} \phi_{\vec{k}}\right],
$$


where $D_{a}=\nabla_{a}-i q A_{a}$ and

$$
m_{k}^{2} \equiv m^{2}+\frac{k^{2} \ell^{2}}{r_{\star}^{2}}=m^{2}+\frac{k^{2} z_{\star}^{2}}{\ell^{2}} \equiv m^{2}+\tilde{m}^{2} .
$$

The indices $a, b$ only run over $t$ and $\zeta$. From the action, one can obtain the equations of motion for the complex scalar,

$$
\left[g^{a b}\left(\nabla_{a}-i q A_{a}\right)\left(\nabla_{b}-i q A_{b}\right)-m_{\vec{k}}^{2}\right] \phi_{\vec{k}}=0,
$$

which can be written as

$$
\left(\partial_{\zeta}^{2}-\partial_{t}^{2}+\frac{2 i q e_{d}}{\zeta} \partial_{t}+\frac{q^{2} e_{d}^{2}}{\zeta^{2}}-\frac{m_{\vec{k}}^{2} \ell_{2}^{2}}{\zeta^{2}}\right) \phi=0,
$$

where $\phi=\phi(t, \zeta)$. For pure $\mathrm{AdS}_{2}$ case as in Eq. (B5), one has $\tilde{m}$. By doing Fourier transformation from the time coordinate to the frequency space, the equation becomes,

$$
\left[\partial_{\zeta}^{2}-\frac{m_{\vec{k}}^{2} \ell_{2}^{2}}{\zeta^{2}}+\left(\omega \pm \frac{q e_{d}}{\zeta}\right)^{2}\right] \phi(\omega, \zeta)=0,
$$

where the \pm sign corresponds to out/in going waves $\left(\partial_{t} \rightarrow \mp i \omega\right.$ and $\left.\partial_{x} \rightarrow \pm i k\right)$, respectively.

\section{b. IR correlation functions of scalars}

Firstly, let's consider the case in the infinite boundary conditions,

$$
d \geq 2: \zeta \sim \frac{z_{\star}}{d(d-1)} \rightarrow 0, \quad d=1: z \rightarrow 0 .
$$

The EOM is dominated by the singularity at $z_{\star} \rightarrow 0$,

$$
\left(\partial_{\zeta}^{2}-\frac{m_{\vec{k}}^{2} \ell_{2}^{2}-q^{2} e_{d}^{2}}{\zeta^{2}}\right) \phi(\omega, \zeta)=0
$$

The asymptotic behavior of the solution is

$$
\begin{aligned}
\phi(\omega, \zeta) & =A(\omega) \zeta^{\frac{1}{2}-\nu_{k}}[1+O(\zeta)]+B(\omega) \zeta^{\frac{1}{2}+\nu_{k}}[1+O(\zeta)] \\
& \sim A(\omega) \zeta^{\Delta_{-}^{\mathrm{IR}}}+B(\omega) \zeta^{\Delta_{+}^{\mathrm{IR}}}, \quad \zeta \rightarrow 0, \quad(\mathrm{C} 56)
\end{aligned}
$$

where the two exponents are, respectively,

$$
\Delta_{ \pm}^{\mathrm{IR}}=\frac{1}{2} \pm \nu_{k}, \quad \nu_{k}=\sqrt{\frac{1}{4}+m_{k}^{2} \ell_{2}^{2}-q^{2} e_{d}^{2}}
$$

For pure $\mathrm{AdS}_{2}$ case in $(1+1)$-dimensional spacetime, one has $\ell_{2}=1, k=0$, and $e_{d}=\mu$. Thus,

$$
\Delta_{ \pm}^{\mathrm{IR}}=\frac{1}{2} \pm \sqrt{\frac{1}{4}+m^{2}-q^{2} \mu^{2}} .
$$

It is worthy of emphasizing that it is possible that the $\nu_{k}$ become pure imaginary, once the electric charge $q$ becomes sufficiently large. Then, $\nu_{k}$ becomes imaginary for sufficiently small $k^{2}<k_{0}^{2}$ (for a given $m$, this always occurs for a sufficiently large $q$ ) with

$$
\begin{aligned}
& \nu_{k}=-i \lambda_{k}, \quad d \geq 2: \lambda_{k}=\frac{\ell_{2} \ell}{r_{\star}} \sqrt{k_{0}^{2}-k^{2}}, \\
& d=1: \lambda=\sqrt{\mu_{L}^{2}-m^{2}-\frac{1}{4}},
\end{aligned}
$$

where

$$
\begin{aligned}
k_{0}^{2} & \equiv \frac{r_{\star}^{2}}{\ell^{2}}\left(\frac{q^{2} e_{d}^{2}-\frac{1}{4}}{\ell_{2}^{2}}-m^{2}\right) \\
& =\frac{d(d-1)}{z_{\star}^{2}}\left(\left(\mu_{L}^{2}-m^{2}\right) \ell_{2}^{2}-\frac{1}{4}\right)>0,
\end{aligned}
$$

where $z_{\star} \equiv \ell^{2} / r_{\star}$ and the local chemical potential $\mu_{L}$ is defined as in Eq. (C63). By observing the conformal dimension in neutral background as in Eq. (C21). It can be viewed that the background electric field acts through the charge as an effective negative mass square, which make it possible that the total mass square $m^{2}-q^{2} \mu^{2}$ becomes negative and resulting in an imaginary conformal dimension.

For a neutral scalar operator with $q=0$ in $\mathrm{AdS}_{d+1}$ spacetime, one can obtain the oscillatory region mass window,

$$
-\frac{d^{2}}{4}<m^{2} \ell^{2}<-\frac{d(d-1)}{4},
$$

where the lower limit comes from the stability of vacuum theory, i.e., Breitenlohner-Freedman (BF) bound of $\mathrm{AdS}_{d+1}$ and the upper limit comes from the condition $k_{0}^{2}>0$. For the $\mathrm{AdS}_{2}$ case, one has

$$
\operatorname{AdS}_{2}:-\frac{1}{4}<m^{2} \ell^{2}<0
$$

By using Eq. (C37), we obtain the chemical potential $\mu_{L}$ for a local observer with charge $q$ in the bulk (the spatial part is flat),

$$
\begin{aligned}
d \geq 2: \mu_{L} & =q \sqrt{g^{t t}} A_{t}=q \frac{\zeta}{\ell_{2}} \frac{e_{d}}{\zeta}=\frac{q e_{d}}{\ell_{2}}, \\
d & =1: \mu_{L}=q \mu,
\end{aligned}
$$

when this local chemical potential exceeds the mass of a charged particle,

$$
\begin{aligned}
& d \geq 2: \mu_{L}^{2}=\frac{q^{2} e_{d}^{2}}{\ell_{2}^{2}}>m^{2}, \\
& d=1: \mu_{L}^{2}=q^{2} \mu^{2}>m^{2},
\end{aligned}
$$


the system will be the Bose-Einstein condensation. This gives the bulk origins of the scalar instabilities in the parameter region in Eq. (C60). Physically, in the near horizon region of the extremal black brane geometry $(T=0)$, at each point in the bulk geometry, e.g., $\mathrm{AdS}_{2} \times \mathbb{R}^{d-1}$, there is a local $d$-dimensional Fermi surface with Fermi momentum $k_{0}$, which upon projection to the boundary theory, would result in a $(d-1)$-dimensional Fermi disc, in which there are gapless excitations at each point in the interior of the disc in the $(d-1)$-dimensional momentum space. For a pure $\mathrm{AdS}_{2}$ bulk vacuum spacetime in $(1+1)$-dimensional spacetime, if $\mu_{L}=q \mu>m$ there will be Bose-Einstein condensation for scalar bosons, while for fermions there is no Fermi surface, since by definition $k_{0}=0$, while there is still a critical chemical potential

$$
\mu_{L}^{c}=\sqrt{m^{2}+\frac{1}{4}}
$$

at which, the NFL phase (since $\nu_{k}<1 / 2$ ) of $\mathrm{CFT}_{1}$ is separated into steady and oscillatory due to the charge instability.

It is worthy of noticing that at $\nu_{k}=0$, or equivalently

$m_{k}=\frac{1}{\ell_{2}} \sqrt{q^{2} e_{d}^{2}-\frac{1}{4}}=\sqrt{\mu_{L}^{2}-\frac{d(d-1)}{4 \ell^{2}}}, \quad \Delta_{ \pm}^{\mathrm{IR}}=\frac{1}{2}$,

which is impossible for neutral black brane with $q=0$, since $m_{k}>0$ is always positive unless $m=0$ and $k=0$ (no scalar at all). As a result, the original two independent solutions are degenerate. In this case, the equation of motion of charged scalar in the infinite boundary conditions at IR fixed point in Eq. (C55) become

$$
\left(\partial_{\zeta}^{2}-\frac{\nu_{k}^{2}-1 / 4}{\zeta^{2}}\right) \phi=\left(\partial_{\zeta}^{2}+\frac{1}{4 \zeta^{2}}\right) \phi=0,
$$

where $\phi=\phi(\omega, \zeta)$. The asymptotic behavior of the solution are

$$
\phi(\omega, \zeta) \sim A(\omega) \zeta^{\frac{1}{2}} \ln \sqrt{\zeta}+B(\omega) \zeta^{\frac{1}{2}}, \quad \zeta \rightarrow 0,
$$

where $\zeta>0$. Secondly, let's consider a more generic case of the solution. In frequency space $\phi(t, \zeta)=e^{-i \omega t} \phi(\omega, \zeta)$, the equation of the motion for a charged scalar $\phi$ can be written as

$$
\left[\partial_{\zeta}^{2}-V(\zeta)\right] \phi=0, \quad V(\zeta) \equiv\left[\frac{m_{\vec{k}}^{2} \ell_{2}^{2}}{\zeta^{2}}-\left(\omega \pm \frac{q e_{d}}{\zeta}\right)^{2}\right]
$$

where the plus and minus sign corresponds to the out/in going waves $\left(\partial_{t} \rightarrow \mp i \omega\right)$. Note that $\omega$ can be scaled away by redefining $\zeta$, reflecting the scaling symmetry of the background solution. the potential can be expressed as a function depending on the dimension of operators in the infinite boundary conditions,

$$
V(\zeta)=\frac{\nu_{k}^{2}-\frac{1}{4}}{\zeta^{2}}-\omega^{2} \mp \frac{2 q e_{d}}{\zeta} \omega,
$$

where in the last identity, we have used the Eq. (C57). According to the asymptotic behavior of the solution in Eq. (C56) and the EOMs above, it is obviously that the frequency $\omega$ dependence in the potential $V(\zeta)$, can be scaled away from by redefining the $\zeta \rightarrow q e_{d} / \omega$ so that the EOMs recovers Eq. (C67). Thus, the solution to the generic case will be of form

$\phi=B\left(\frac{\zeta}{\omega}\right)^{\frac{1}{2}+\nu_{k}}[1+O(\zeta)]+A\left(\frac{\zeta}{\omega}\right)^{\frac{1}{2}-\nu_{k}}[1+O(\zeta)]$,

where as $\zeta \rightarrow 0$, one has

$$
B \sim \omega^{\frac{1}{2}+\nu_{k}}, \quad A \sim \omega^{\frac{1}{2}-\nu_{k}} .
$$

Thus after imposing the infalling boundary condition on $\phi$, the correlated functions are expected to be of form

$$
\mathcal{G}_{R} \propto \frac{B}{A} \sim \omega^{2 \nu_{k}}
$$

This implies a coordinate space correlation function by doing an inverse Fourier transformation, ${ }^{1}$

$\mathcal{G}^{R}(t)=-\frac{1+\operatorname{sgn}(t)}{|t|^{1+2 \nu_{k}}} \frac{e^{i \pi \nu_{k}}}{\sqrt{2 \pi}} \Gamma\left[1+2 \nu_{k}\right] \sin \left(2 \pi \nu_{k}\right) \sim \frac{1}{t^{2 \Delta}}$,

with the conformal dimension $\Delta$ of the boson operator $\mathcal{O}_{B}$ given by $\Delta=1 / 2+\nu_{k}$, which is nothing but $\Delta_{+}^{\mathrm{IR}}$ defined in Eq. (C57). It is worthy of noticing that the dimension $\Delta$ depends on the charge $q$ through $\nu_{k}$. In particular, it is possible for $\nu_{k}$ to become imaginary when the charge $q$ becomes sufficiently large. This implies that in the constant electric field $A_{t}=e_{d} / \zeta$ with sufficiently large charge $q$ can be pair produced, which cause an instability for scalars. When $\nu_{k}$ is imaginary, there is an ambiguity in specifying $\mathcal{G}_{R}$ since one can choose either $A$ or $B$ as the source term.

\footnotetext{
${ }^{1}$ Note: for $\mathcal{G}_{k}^{R} \sim|\omega|^{2 \nu_{k}}$, the inverse Fourier transformation gives the result $-\sqrt{2 / \pi}|t|^{-1-2 \nu_{k}} \Gamma\left[1+2 \nu_{k}\right] \sin \left(\pi \nu_{k}\right) \sim t^{-2 \Delta}$.
} 
The solution turns out to be ${ }^{2}$

\section{c. Zero temperature case}

$$
\begin{aligned}
& \phi(\zeta)=C_{1} M_{i q e_{d},-\nu_{k}}(2 i \omega \zeta)+C_{2} W_{i q e_{d},-\nu_{k}}(2 i \omega \zeta), \quad \text { in going } e^{+i \omega t-i k x}\left(\partial_{t} \rightarrow+i \omega\right) \\
& \phi(\zeta)=C_{1} M_{-i q e_{d}, \nu_{k}}(2 i \omega \zeta)+C_{2} W_{-i q e_{d}, \nu_{k}}(2 i \omega \zeta), \quad \text { out going } e^{-i \omega t+i k x}\left(\partial_{t} \rightarrow-i \omega\right)
\end{aligned}
$$

where $M_{k, \nu}(z)$ and $W_{k, \nu}$ are both the Whittaker functions, which can be converted to be Kummer confluent hypergeometric function $F_{1}(a, b ; z)$ and confluent hypergeometric function $U(a, b ; z)$, respectively, as below,

$$
M_{k, \nu}(z)=e^{-z / 2} z^{1 / 2+\nu} F_{1}\left(\frac{1}{2}+\nu-k, 1+2 \nu, z\right), \quad W_{k, \nu}(z)=e^{-z / 2} z^{\nu+1 / 2} U\left(\frac{1}{2}+\nu-k, 1+2 \nu, z\right) .
$$

$M_{k, m}(z)$ is zero at $z=0$ for $m>0$, while $W_{k, m}(z)$ is infinite at $z=0$ for integer $m>0$, and has a branch cut discontinuity in the complex $z$ plane running from $-\infty$ to 0 . The Kummer confluent hypergeometric function $F_{1}(a, b, z) \equiv{ }_{1} F_{1}(a, b ; z)$, has the series expansion

$$
F_{1}(a, b, z)=\sum_{k=0}^{\infty} \frac{(a)_{k}}{(b)_{k}} \frac{z^{k}}{k !}=1+\frac{a}{b} z+\frac{a(a+1)}{2 b(b+1)} z^{2}+O\left(z^{3}\right),
$$

and the confluent hypergeometric function $U(a, b, z)$ has the integral representation

$$
U(a, b, z)=\frac{1}{\Gamma[a]} \int_{0}^{\infty} e^{-z t} t^{a-1}(1+t)^{b-a-1} d t=\frac{1}{z^{a}}\left(1-\frac{a(1+a-b)}{z}+\frac{a(1+a)(1+a-b)(2+a-b)}{2 z^{2}}+O\left(\frac{1}{z^{3}}\right)\right) .
$$

Therefore in the $z \rightarrow \infty$ expansion, one has $M_{k, \nu}(z) \sim e^{\frac{z}{2}} z^{-\nu}, W_{k, \nu}(z) \sim e^{-\frac{z}{2}} z^{\nu}$, which basically implies that Whittaker $M_{k, \nu}(z)$ is divergent in the large $z$. In the near horizon region $\zeta \rightarrow \infty$ for the out going wave $e^{-i \omega t+i k x}\left(\partial_{t} \rightarrow-i \omega\right)$, the asymptotic

$$
M_{-i q e_{d}, \nu_{k}}(2 i \omega \zeta) \sim e^{i \omega \zeta}(2 i \omega \zeta)^{i q e_{d}}, \quad W_{-i q e_{d}, \nu_{k}}(2 i \omega \zeta) \sim e^{-i \omega \zeta}(2 i \omega \zeta)^{-i q e_{d}}+\cdots
$$

Thus, in the near horizon region,

$$
\phi \rightarrow C_{1} e^{-i \omega t} e^{i \omega \zeta} \zeta^{i q e_{d}}+C_{2} e^{-i \omega t} e^{-i \omega \zeta} \zeta^{-i q e_{d}} \sim C_{1} e^{-i \omega\left(t-\zeta-\frac{q e_{d}}{\omega} \ln \zeta\right)}+C_{2} e^{-i\left(\omega+\zeta+\frac{q e_{d}}{\omega} \ln \zeta\right)}, \quad(\zeta \rightarrow \infty)
$$

and it is obviously that the $M$-Whittaker function associated with $C_{1}$ is an outgoing solution, while the $W$-Whittaker function associated with $C_{2}$ is always an in-going solution. Therefore, in the following, we will only keep the in-going part from $W$ by setting $C_{1}=0$. In the infinite boundary condition $\zeta \rightarrow 0$, the Whittaker function can be expanded as

$$
\begin{aligned}
& M_{-i q e_{d}, \nu_{k}}(2 i \omega \zeta) \sim(2 i \omega \zeta)^{\frac{1}{2}+\nu_{k}}, \\
& W_{-i q e_{d}, \nu_{k}}(2 i \omega \zeta) \sim(2 i \omega \zeta)^{\frac{1}{2}-\nu_{k}} \frac{\Gamma\left[2 \nu_{k}\right]}{\Gamma\left[\frac{1}{2}+\nu_{k}+i q e_{d}\right]}+(2 i \omega \zeta)^{\frac{1}{2}+\nu_{k}} \frac{\Gamma\left[-2 \nu_{k}\right]}{\Gamma\left[\frac{1}{2}-\nu_{k}+i q e_{d}\right]},
\end{aligned}
$$

from which we have

$$
\mathcal{G}_{k}(\omega) \equiv \frac{B(\omega)}{A(\omega)}=(2 i \omega)^{2 \nu_{k}} \frac{\Gamma\left[-2 \nu_{k}\right]}{\Gamma\left[2 \nu_{k}\right]} \frac{\Gamma\left[\frac{1}{2}+\nu_{k}+i q e_{d}\right]}{\Gamma\left[\frac{1}{2}-\nu_{k}+i q e_{d}\right]}, \quad\left(\partial_{t} \rightarrow-i \omega\right),
$$

which corresponds to the advanced Green's function associated with the outgoing modes. If one chooses the ingoing wave conversion, $\left(\partial_{t} \rightarrow i \omega\right)\left(\phi \sim e^{i \omega t-i k x}\right)$, then in the infinite boundary condition $\zeta \rightarrow 0$, the Whittaker function can be expanded as

\footnotetext{
${ }^{2}$ We choose the solution $\phi(\zeta)=C_{1} M_{-i q e_{d}, \nu_{k}}(2 i \omega \zeta)+C_{2} W_{-i q e_{d}, \nu_{k}}(2 i \omega \zeta)$, in/out going $\left(\partial_{t} \rightarrow \pm i \omega\right)$, which corresponds to $\omega+$ $q e_{d} / \zeta$ in the potential.
} 


$$
\begin{aligned}
M_{i q e_{d}, \nu_{k}}(2 i \omega \zeta)= & (2 i \omega \zeta)^{\frac{1}{2}+\nu_{k}}\left(1-\frac{2 i \omega \zeta}{1+2 \nu_{k}}(2 i \omega \zeta)+\cdots\right), \\
W_{i q e_{d}, \nu_{k}}(2 i \omega \zeta)= & (2 i \omega \zeta)^{\frac{1}{2}+\nu_{k}} \frac{\Gamma\left[-2 \nu_{k}\right]}{\Gamma\left[\frac{1}{2}-i q e_{d}-\nu_{k}\right]} \\
& +(2 i \omega \zeta)^{\frac{1}{2}-\nu_{k}} \frac{\Gamma\left[2 \nu_{k}\right]}{\Gamma\left[\frac{1}{2}-i q e_{d}+\nu_{k}\right]}+\cdots .
\end{aligned}
$$

The $M$-Whittaker function is always vanishing at infinite boundary condition, while the $W$-Whittaker has both positive and negative branches labeled by dimensions of operator $\Delta_{ \pm}^{\mathrm{IR}}$. Thus, the asymptotic behavior of the complex scalar $\phi$ at $\zeta \rightarrow 0$ is given by

$$
\begin{aligned}
\phi \sim & (2 i \omega \zeta)^{\Delta_{+}^{\mathrm{R}}} \frac{\Gamma\left[-2 \nu_{k}\right]}{\Gamma\left[\frac{1}{2}-i q e_{d}-\nu_{k}\right]} \\
& +(2 i \omega \zeta)^{\Delta_{-}^{\mathrm{IR}}} \frac{\Gamma\left[2 \nu_{k}\right]}{\Gamma\left[\frac{1}{2}-i q e_{d}+\nu_{k}\right]} \sim B \zeta^{\nu_{k}+\frac{1}{2}}+A \zeta^{-\nu_{k}+\frac{1}{2}}
\end{aligned}
$$

Then by comparing with Eq. (C56), one can read the scalar correlation function at the IR fixed point,

$\mathcal{G}_{k}(\omega) \equiv \frac{B(\omega)}{A(\omega)}=(2 i \omega)^{2 \nu_{k}} \frac{\Gamma\left[-2 \nu_{k}\right]}{\Gamma\left[2 \nu_{k}\right]} \frac{\Gamma\left[\frac{1}{2}+\nu_{k}-i q e_{d}\right]}{\Gamma\left[\frac{1}{2}-\nu_{k}-i q e_{d}\right]}$,

where we have used the notation $\left(\partial_{t} \rightarrow i \omega\right)$. It corresponds to the retarded Greens' function associated with the infalling modes. In summary, the retarded scalar function of the IR CFT is given by $[75,76]$

$\mathcal{G}_{k}^{R}(\omega)=(-i)^{2 \nu_{k}} \frac{\Gamma\left[-2 \nu_{k}\right]}{\Gamma\left[2 \nu_{k}\right]} \frac{\Gamma\left[\frac{1}{2}-i q e_{d}+\nu_{k}\right]}{\Gamma\left[\frac{1}{2}-i q e_{d}-\nu_{k}\right]}(2 \omega)^{2 \nu_{k}}$,

$(-i)^{2 \nu_{k}}=e^{-i \pi \nu_{k}}$

which has the form of the retarded two-point function of a scalar operator in a $(1+0)$-dimensional conformal quantum mechanics $\mathrm{CFT}_{1}$. The advanced Green's function is given by

$$
\begin{aligned}
\mathcal{G}_{k}^{A}(\omega) & =(i)^{2 \nu_{k}} \frac{\Gamma\left[-2 \nu_{k}\right]}{\Gamma\left[2 \nu_{k}\right]} \frac{\Gamma\left[\frac{1}{2}+i q e_{d}+\nu_{k}\right]}{\Gamma\left[\frac{1}{2}+i q e_{d}-\nu_{k}\right]}(2 \omega)^{2 \nu_{k}}, \\
(i)^{2 \nu_{k}} & =e^{i \pi \nu_{k}} .
\end{aligned}
$$

For a scalar, from the correlator functions above, one finds that

$$
\begin{aligned}
\frac{\mathcal{G}^{R}(\omega)}{\mathcal{G}^{A}(\omega)} & =\frac{c(k)}{c^{\star}(k)}=e^{-2 \pi i \nu_{k}} \frac{\cos \left[\pi\left(\nu_{k}+i q e_{d}\right)\right]}{\cos \left[\pi\left(\nu_{k}-i q e_{d}\right)\right]} \equiv e^{2 i \gamma_{k}} \\
& =\frac{e^{-2 \pi \nu_{k} i}+e^{-2 \pi q e_{d}}}{e^{2 \pi \nu_{k} i}+e^{-2 \pi q e_{d}}}=\frac{\left(e^{-2 \pi \nu_{k} i}+e^{-2 \pi q e_{d}}\right)^{2}}{\left|e^{2 \pi \nu_{k} i}+e^{-2 \pi q e_{d}}\right|^{2}},
\end{aligned}
$$

where we have introduced the notation that

$$
\mathcal{G}^{R}(\omega) \equiv c(k) \omega^{2 \nu_{k}}, \quad c(k) \equiv|c(k)| e^{i \gamma_{k}},
$$

with $c(k)$ denoting the prefactor,

$$
\begin{aligned}
c(k) & \equiv(-i)^{2 \nu_{k}} c\left(\nu_{k}\right)=(-i)^{2 \nu_{k}}\left|c\left(\nu_{k}\right)\right| e^{i \theta_{k}}, \\
c^{\star}(k) & \equiv i^{2 \nu_{k}} c^{\star}\left(\nu_{k}\right)=i^{2 \nu_{k}}\left|c\left(\nu_{k}\right)\right| e^{-i \theta_{k}},
\end{aligned}
$$

where $\theta_{k}$ is the phase of $c\left(\nu_{k}\right)$ :

$c\left(\nu_{k}\right)=\left|c\left(\nu_{k}\right)\right| e^{i \theta_{k}}=2^{2 \nu_{k}} \frac{\Gamma\left[-2 \nu_{k}\right]}{\Gamma\left[2 \nu_{k}\right]} \frac{\Gamma\left[\frac{1}{2}-i q e_{d}+\nu_{k}\right]}{\Gamma\left[\frac{1}{2}-i q e_{d}-\nu_{k}\right]}$.

Thus, by writing $\mathcal{G}^{R}(\omega)=c\left(\nu_{k}\right)(-i \omega)^{2 \nu_{k}}$ and $\mathcal{G}_{A}(\omega)=$ $c^{\star}\left(\nu_{k}\right)(i \omega)^{2 \nu_{k}}$, then

$$
\frac{\mathcal{G}^{R}(\omega)}{\mathcal{G}^{A}(\omega)}=e^{-2 \pi i \nu_{k}} \frac{c\left(\nu_{k}\right)}{c^{\star}\left(\nu_{k}\right)}=e^{-2 \pi i \nu_{k}} e^{2 i \theta_{k}},
$$

for real $\nu_{k}$, the equation in Eq. (C89) gives the phase of $c\left(\nu_{k}\right)$, and for imaginary $\nu_{k}$, the equation gives the modulus of $c\left(\nu_{k}\right)$. Therefore,

$$
e^{2 i \theta_{k}}=\frac{\cos \left[\pi\left(\nu_{k}+i q e_{d}\right)\right]}{\cos \left[\pi\left(\nu_{k}-i q e_{d}\right)\right]}=e^{2 i\left(\gamma_{k}+\pi \nu_{k}\right)},
$$

from which one can obtain the quantity related to the effective IR charge parameter [55],

$$
e^{2 \pi q e_{d}}=-\frac{\sin (\pi \Delta+\theta)}{\sin (\pi \Delta-\theta)},
$$

where $\theta \equiv-\pi / 2-\theta_{k} \in(\pi \Delta, \pi(1-\Delta))$ for the boson. The dimensionless, temperature-independent effective IR coupling parameter $e_{d}$ can be redefined to be related to the frequency shift $\omega_{s}$ at finite temperature as [55]

$$
\omega_{s} \equiv 2 \pi q e_{d} T \text {. }
$$

(1) For real $\nu_{k}$ : The ratios $G^{R}(\omega) / G^{A}(\omega)$ become a pure phase and one find that

$$
\gamma_{k}=\arg \left[\Gamma\left[-2 \nu_{k}\right]\left(e^{-2 \pi \nu_{k} i}+e^{-2 \pi q e_{d}}\right)\right] .
$$

The factor $e^{i \gamma_{k}}$ and thus $c(k)$ always lies in the upper-half complex plane, while for scalars $e^{i \gamma_{k}+2 \pi \nu_{k} i}$ always lies in the lower-half complex plane. Namely, for $\nu_{k} \in(0,1 / 2)$,

$$
\gamma_{k}+2 \pi \nu_{k}>\pi, \quad \Rightarrow \pi-\gamma_{k}<2 \pi \nu_{k} .
$$


(2) For pure imaginary $\nu_{k}=-i \lambda_{k}\left(\lambda_{k}>0\right)$, the ratio becomes real and give the modulus of $c(k)$.

$$
e^{-4 \pi \lambda_{k}}<\frac{e^{-2 \pi \lambda_{k}}+e^{-2 \pi q e_{d}}}{e^{2 \pi \lambda_{k}}+e^{-2 \pi q e_{d}}}=e^{2 i \gamma_{k}}<1 .
$$

(3) For generic $\nu_{k}, \mathcal{G}_{k}(\omega)$ and $G^{R}(\omega, k)$ have a logarithmic branch point at $\omega=0$. One can choose the branch cut along the negative imaginary axis, i.e., the physical sheet to be $\theta \in(-\pi / 2,3 \pi / 2)$, which resolves into a line of poles along the branch cut when going to finite temperature.

\section{d. Finite Temperature case}

For a charged scalar field at finite temperature, the near horizon region is a charged black brane in $\mathrm{NAdS}_{2} \times \mathbb{R}^{d-1}$ spacetime at finite charge density, the background metric and gauge field are given by Eq. (C36)

$$
\begin{aligned}
d s^{2} & =\frac{\ell_{2}^{2}}{\zeta^{2}}\left[-\left(1-\frac{\zeta^{2}}{\zeta_{0}^{2}}\right) d t^{2}+\left(1-\frac{\zeta^{2}}{\zeta_{0}^{2}}\right)^{-1} d \zeta^{2}\right]+\frac{r_{\star}^{2}}{\ell^{2}} d x^{2}, \\
A_{t}(\zeta) & =e_{d}\left(\frac{1}{\zeta}-\frac{1}{\zeta_{0}}\right)=\frac{e_{d}}{\zeta}\left(1-\frac{\zeta}{\zeta_{0}}\right),
\end{aligned}
$$

where the physical coordinates are listed as below

$$
\begin{array}{cl}
\text { IR horizon } \quad r_{\star} \leq r_{0} \leq r<\infty, \quad \text { UV boundary } \\
\\
z_{\star} \geq z_{0} \geq z>0, \\
\zeta_{\star} \geq \zeta_{0} \geq \zeta>0,
\end{array}
$$

where the coordinates with $\star, 0$ and no subscript correspond to the radius for the zero black brane (extreme one), finite temperature black brane, and ordinary black brane, respectively. By definition, we have

$$
\zeta \equiv \frac{z_{\star}^{2}}{d(d-1)\left(z_{\star}-z\right)}>0, \quad \zeta_{0} \equiv \frac{z_{\star}^{2}}{d(d-1)\left(z_{\star}-z_{0}\right)},
$$

where $z \equiv \ell^{2} / r$. In this case, the finite temperature with respect to $\tau$ is defined by

$$
T=\frac{1}{2 \pi \zeta_{0}}
$$

The scalar action in the background is given by Eq. (C44), from which, one can obtain the equations of motion for the complex scalar as in Eq. (C45), which explicitly become

$$
\left[\left(1-\frac{\zeta^{2}}{\zeta_{0}^{2}}\right)^{2} \partial_{\zeta}^{2}-\partial_{t}^{2}+\frac{\ell^{2} \ell_{2}^{2}}{r_{\star}^{2} \zeta^{2}}\left(1-\frac{\zeta^{2}}{\zeta_{0}^{2}}\right) \partial_{x}^{2}+\frac{2 i q e_{d}}{\zeta}\left(1-\frac{\zeta}{\zeta_{0}}\right) \partial_{t}-\frac{2 \zeta}{\zeta_{0}^{2}}\left(1-\frac{\zeta^{2}}{\zeta_{0}^{2}}\right) \partial_{\zeta}+\frac{q^{2} e_{d}^{2}}{\zeta^{2}}\left(1-\frac{\zeta}{\zeta_{0}}\right)^{2}-\frac{m^{2} \ell_{2}^{2}}{\zeta^{2}}\left(1-\frac{\zeta^{2}}{\zeta_{0}^{2}}\right)\right]
$$

$\phi=0$.

where $\phi=\phi(\zeta, t, x)$. In the momentum space(out/in going: $\partial_{t} \rightarrow \mp i \omega, \partial_{x} \rightarrow \pm i k$ ), the EOM can be reexpressed as

$$
\left(1-\frac{\zeta^{2}}{\zeta_{0}^{2}}\right) \partial_{\zeta}\left[\left(1-\frac{\zeta^{2}}{\zeta_{0}^{2}}\right) \partial_{\zeta} \phi\right]+\left[\omega \pm \frac{q e_{d}}{\zeta}\left(1-\frac{\zeta}{\zeta_{0}}\right)\right]^{2} \phi-\left(m^{2}+\frac{k^{2} \ell^{2}}{r_{\star}^{2}}\right) \frac{\ell_{2}^{2}}{\zeta^{2}}\left(1-\frac{\zeta^{2}}{\zeta_{0}^{2}}\right) \phi=0,
$$

where $\phi=\phi(\zeta, t, x)$. In the infinite boundary conditions $z \rightarrow 0, \zeta \rightarrow z_{\star} / d(d-1) \ll 1$, the EOM is dominated by the singularity at $z_{\star} \rightarrow 0$,

$$
\left(\partial_{\zeta}^{2}-\frac{m_{\vec{k}}^{2} \ell_{2}^{2}-q^{2} e_{d}^{2}}{\zeta^{2}}\right) \phi(\zeta)=0
$$


where for simplicity we have denoted $\phi(\zeta)=\phi(\zeta, \omega, k)$ and the equation of motion is just the same as that in Eq. (C55). The asymptotic behavior of the solution is the same as that in Eq. (C56) with exponent indexes like those in Eq. (C57). The full EOM above can be simplified as

$$
\partial_{\zeta}^{2} \phi(\zeta)+\frac{2 \zeta}{\zeta^{2}-\zeta_{0}^{2}} \partial_{\zeta} \phi(\zeta)+\left(-\frac{m_{k}^{2} \ell_{2}^{2}}{\zeta^{2}\left(1-\frac{\zeta^{2}}{\zeta_{0}^{2}}\right)}+\frac{\left[\omega \mp q e_{d}\left(\frac{1}{\zeta}-\frac{1}{\zeta_{0}}\right)\right]^{2}}{\left(1-\frac{\zeta^{2}}{\zeta_{0}^{2}}\right)^{2}}\right) \phi(\zeta)=0,
$$

where $m_{k}^{2}$ is define in Eq. (C50) and $\nu_{k}$ is defined in Eq. (C57). In the following, we will chose the out-going wave conversion $\partial_{t} \rightarrow-i \omega$. The two linearly independent solutions turns out to be

$$
\begin{aligned}
\phi(\zeta)= & \left(\frac{\zeta+\zeta_{0}}{\zeta-\zeta_{0}}\right)^{\frac{i \omega \zeta_{0}}{2}}\left[C_{1}\left(1+\frac{\zeta_{0}}{\zeta}\right)^{-\frac{1}{2}-\nu_{k}}{ }_{2} F_{1}\left(\frac{1}{2}+\nu_{k}-i q e_{d}, \frac{1}{2}+\nu_{k}+i q e_{d}-i \omega \zeta_{0} ; 1+2 \nu_{k} ; \frac{2 \zeta}{\zeta+\zeta_{0}}\right),\right. \\
& \left.+C_{2}(-2)^{-2 \nu_{k}}\left(1+\frac{\zeta_{0}}{\zeta}\right)^{-\frac{1}{2}+\nu_{k}}{ }_{2} F_{1}\left(\frac{1}{2}-\nu_{k}-i q e_{d}, \frac{1}{2}-\nu_{k}+i q e_{d}-i \omega \zeta_{0} ; 1-2 \nu_{k} ; \frac{2 \zeta}{\zeta+\zeta_{0}}\right)\right] .
\end{aligned}
$$

Or, the two linearly independent solutions turns out to be

$$
\begin{aligned}
\phi(\zeta)= & C_{1}\left(1-\frac{\zeta_{0}}{\zeta}\right)^{-\frac{1}{2}+\nu_{k}}\left(\frac{\zeta+\zeta_{0}}{\zeta-\zeta_{0}}\right)^{i q e_{d}-\frac{i \omega \zeta_{0}}{2}}{ }_{2} F_{1}\left(\frac{1}{2}-\nu_{k}+i q e_{d}, \frac{1}{2}-\nu_{k}+i q e_{d}-i \zeta_{0} \omega ; 1-2 \nu_{k} ; \frac{2 \zeta}{\zeta-\zeta_{0}}\right) \\
& +C_{2}\left(1-\frac{\zeta_{0}}{\zeta}\right)^{-\frac{1}{2}-\nu_{k}}\left(\frac{\zeta+\zeta_{0}}{\zeta-\zeta_{0}}\right)^{i q e_{d}-\frac{i \omega \zeta_{0}}{2}}{ }_{2} F_{1}\left(\frac{1}{2}+\nu_{k}+i q e_{d}, \frac{1}{2}+\nu_{k}+i q e_{d}-i \zeta_{0} \omega ; 1+2 \nu_{k} ; \frac{2 \zeta}{\zeta-\zeta_{0}}\right)
\end{aligned}
$$

where the hypergeometric function is defined by

$$
{ }_{2} F_{1}(a, b, ; c, ; z)=\sum_{k=0}^{\infty} \frac{(a)_{k}(b)_{k}}{(c)_{k}} \frac{z^{k}}{k !}=1+\frac{a b}{c} z+\frac{a(1+a) b(1+b)}{2 c(1+c)} z^{2}+O\left(z^{3}\right) .
$$

In the near horizon limit, $\zeta \rightarrow \zeta_{0}$, the special function associated with the coefficients $C_{1}$ and $C_{2}$ in Eq. (C105), respectively, have the following expansion

$$
\begin{aligned}
{ }_{2} F_{1} & \left(\frac{1}{2}+\nu_{k}-i q e_{d}, \frac{1}{2}+\nu_{k}+i q e_{d}-i \omega \zeta_{0} ; 1+2 \nu_{k} ; \frac{2 \zeta}{\zeta+\zeta_{0}}\right) \\
\approx & \frac{i \pi}{\sinh \left(\pi \omega \zeta_{0}\right)} \Gamma\left[1+2 \nu_{k}\right] \\
& \times\left(\frac{\left(\frac{2 \zeta_{0}}{\zeta_{0}-\zeta}\right)-i \omega \zeta_{0}}{\Gamma\left[\frac{1}{2}+\nu_{k}-i q e_{d}\right] \Gamma\left[\frac{1}{2}+\nu_{k}+i q e_{d}-\omega \zeta_{0}\right] \Gamma\left[1+i \omega \zeta_{0}\right]}-\frac{1}{\Gamma\left[\frac{1}{2}+\nu_{k}+i q e_{d}\right] \Gamma\left[\frac{1}{2}+\nu_{k}-i q e_{d}+i \omega \zeta_{0}\right] \Gamma\left[1-i \omega \zeta_{0}\right]}\right), \\
{ }_{2} F_{1}\left(\frac{1}{2}-\nu_{k}-i q e_{d}, \frac{1}{2}-\nu_{k}+i q e_{d}-i \omega \zeta_{0} ; 1-2 \nu_{k} ; \frac{2 \zeta}{\zeta+\zeta_{0}}\right) & \quad \frac{\left(\frac{2 \zeta_{0}}{\zeta_{0}-\zeta}\right)-i \omega \zeta_{0}}{\sinh \left(\pi \omega \zeta_{0}\right)} \Gamma\left[1-2 \nu_{k}\right] \\
& \quad \times\left(\frac{\Gamma\left[\frac{1}{2}-\nu_{k}-i q e_{d}\right] \Gamma\left[\frac{1}{2}-\nu_{k}+i q e_{d}-\omega \zeta_{0}\right] \Gamma\left[1+i \omega \zeta_{0}\right]}{\Gamma\left[\frac{1}{2}-\nu_{k}+i q e_{d}\right] \Gamma\left[\frac{1}{2}-\nu_{k}-i q e_{d}+i \omega \zeta_{0}\right] \Gamma\left[1-i \omega \zeta_{0}\right]}\right)
\end{aligned}
$$

Considering an out wave conversion $\partial_{t} \rightarrow-i \omega$, then it is worthy of noticing that without the expansion wave factor $\left(\frac{2 \zeta_{0}}{\zeta_{0}-\zeta}\right)^{-i \omega \zeta_{0}}$, the solution are pure in-falling wave, since for fixed phase,

$$
e^{-i \omega t}\left(\frac{\zeta_{0}+\zeta}{\zeta_{0}-\zeta}\right)^{\frac{i \omega \zeta_{0}}{2}} \sim e^{-i \omega\left[t-\frac{1}{2} \zeta_{0} \ln \left(\frac{\zeta_{0}+\zeta}{\zeta_{0}-\zeta}\right)\right]} \Rightarrow \zeta=\zeta_{0} \tanh \left(\frac{t}{\zeta_{0}}\right)
$$


While the solution associated with the expansion wave factor will become out-going wave, since

$$
e^{-i \omega t}\left(\frac{\zeta_{0}+\zeta}{\zeta_{0}-\zeta}\right)^{\frac{i \omega \zeta_{0}}{2}}\left(\frac{2 \zeta_{0}}{\zeta_{0}-\zeta}\right)^{-i \omega \zeta_{0}} \sim e^{-i \omega\left[t-\frac{1}{2} \zeta_{0} \ln \left(\zeta_{0}+\zeta\right)\left(\zeta_{0}-\zeta\right)\right]} \Rightarrow \zeta=\sqrt{\zeta_{0}^{2}-e^{\frac{2 t}{\zeta_{0}}}}
$$

Thus, one needs to choose the proper coefficients so that the expansion wave factors are completely canceled and only the in-falling wave solution are present in the near horizon region. It turns out that one has to choose

$$
\begin{aligned}
\frac{C_{1}}{C_{2}} & =-(-1)^{2 \nu_{k}} \frac{\Gamma\left[1-2 \nu_{k}\right]}{\Gamma\left[1+2 \nu_{k}\right]} \frac{\Gamma\left[\frac{1}{2}+\nu_{k}-i q e_{d}\right]}{\Gamma\left[\frac{1}{2}-\nu_{k}-i q e_{d}\right]} \frac{\Gamma\left[\frac{1}{2}+\nu_{k}+i q e_{d}-i \omega \zeta_{0}\right]}{\Gamma\left[\frac{1}{2}-\nu_{k}+i q e_{d}-i \omega \zeta_{0}\right]} \\
& =\frac{\Gamma\left[-2 \nu_{k}\right]}{\Gamma\left[2 \nu_{k}\right]} \frac{\Gamma\left[\frac{1}{2}+\nu_{k}-i q e_{d}\right]}{\Gamma\left[\frac{1}{2}-\nu_{k}-i q e_{d}\right]} \frac{\left.\Gamma \frac{1}{2}+\nu_{k}+i q e_{d}-i \omega \zeta_{0}\right]}{\Gamma\left[\frac{1}{2}-\nu_{k}+i q e_{d}-i \omega \zeta_{0}\right]},
\end{aligned}
$$

where we have used that $\Gamma[1+\alpha]=\alpha \Gamma[\alpha]$ and $(-1)^{2 \nu_{k}}=1$. In this case, the final solution of bulk equation in Eq. (C105) with a pure infalling wave near the horizon becomes,

$$
\begin{aligned}
\phi(\zeta)= & \left(\frac{\zeta+\zeta_{0}}{\zeta-\zeta_{0}}\right)^{\frac{i \omega \zeta_{0}}{2}} C_{2}(-2)^{-2 \nu_{k}}\left[\left(1+\frac{\zeta_{0}}{\zeta}\right)^{-\frac{1}{2}+\nu_{k}}{ }_{2} F_{1}\left(\frac{1}{2}-\nu_{k}-i q e_{d}, \frac{1}{2}-\nu_{k}+i q e_{d}-i \omega \zeta_{0} ; 1-2 \nu_{k} ; \frac{2 \zeta}{\zeta+\zeta_{0}}\right)\right. \\
& \left.+\frac{C_{1}}{C_{2}}(-2)^{2 \nu_{k}}\left(1+\frac{\zeta_{0}}{\zeta}\right)^{-\frac{1}{2}-\nu_{k}}{ }_{2} F_{1}\left(\frac{1}{2}+\nu_{k}-i q e_{d}, \frac{1}{2}+\nu_{k}+i q e_{d}-i \omega \zeta_{0} ; 1+2 \nu_{k} ; \frac{2 \zeta}{\zeta+\zeta_{0}}\right)\right] .
\end{aligned}
$$

In the infinite boundary $\operatorname{condition}\left(z \rightarrow 0, \zeta \rightarrow z_{\star} /(d(d-1)) \ll 1\right)$, the EOM is dominated by

$$
\partial_{\zeta}^{2} \phi(\zeta)+\frac{2 \zeta}{\zeta^{2}-\zeta_{0}^{2}} \partial_{\zeta} \phi(\zeta)+\left(-\frac{m_{k}^{2} \ell_{2}^{2}}{\zeta^{2}\left(1-\frac{\zeta^{2}}{\zeta_{0}^{2}}\right)}+\frac{\left[q e_{d}\left(\frac{1}{\zeta}-\frac{1}{\zeta_{0}}\right)\right]^{2}}{\left(1-\frac{\zeta^{2}}{\zeta_{0}^{2}}\right)^{2}}\right) \phi(\zeta)=0
$$

which give the asymptotic behavior of the solution

$$
\phi(\zeta) \approx C_{3}\left(\frac{\zeta}{\zeta_{0}}\right)^{\frac{1}{2}-\nu_{k}}+C_{4}(-2)^{2 \nu_{k}}\left(\frac{\zeta}{\zeta_{0}}\right)^{\frac{1}{2}+\nu_{k}} \sim(-2)^{-2 \nu_{k}} C_{2}\left(\frac{\zeta}{\zeta_{0}}\right)^{\frac{1}{2}-\nu_{k}}+C_{1}\left(\frac{\zeta}{\zeta_{0}}\right)^{\frac{1}{2}+\nu_{k}}=A(\omega) \zeta^{\Delta_{-}^{\mathrm{IR}}}+B(\omega) \zeta^{\Delta_{+}^{\mathrm{IR}}},
$$

where $\Delta_{ \pm}^{\mathrm{IR}}=1 / 2 \pm \nu_{k}$. On the other hand, in the infinite boundary condition, Eq. (C105) becomes

$$
\phi(\zeta)=(-1)^{\frac{i \omega \zeta_{0}}{2}}\left[C_{1}\left(\frac{\zeta}{\zeta_{0}}\right)^{\frac{1}{2}+\nu_{k}}+C_{2}(-2)^{-2 \nu_{k}}\left(\frac{\zeta}{\zeta_{0}}\right)^{\frac{1}{2}-\nu_{k}}\right]=(-1)^{\frac{i \omega \zeta_{0}}{2}}\left[C_{1} \zeta_{0}^{-\Delta_{+}^{\mathrm{IR}}} \zeta^{\Delta_{+}^{\mathrm{IR}}}+C_{2}(-2)^{-2 \nu_{k}} \zeta_{0}^{-\Delta_{-}^{\mathrm{IR}}} \zeta^{\Delta_{-}^{\mathrm{IR}}}\right]
$$

from which, we have $C_{3} \sim(-2)^{-2 \nu_{k}} C_{2},(-2)^{2 \nu_{k}} C_{4} \sim C_{1}$. Thus, one can read the correlator functions

$$
\mathcal{G}^{A}(\omega, k)=\frac{B(\omega)}{A(\omega)}=\frac{C_{4}}{C_{3}}(-2)^{2 \nu_{k}} \zeta_{0}^{\left(-\Delta_{+}^{\mathrm{IR}}+\Delta_{-}^{\mathrm{IR}}\right)}=\frac{C_{1}}{C_{2}}(-2)^{2 \nu_{k}} \zeta_{0}^{\left(\Delta_{-}^{\mathrm{IR}}-\Delta_{+}^{\mathrm{IR}}\right)}=\frac{C_{1}}{C_{2}}\left(\frac{\zeta_{0}}{2}\right)^{-2 \nu_{k}} .
$$

By using Eq. (C109), we obtain the retarded Green's function as

$$
\mathcal{G}^{T}(\omega, k)=\left(\frac{\zeta_{0}}{2}\right)^{-2 \nu_{k}} \frac{\Gamma\left[-2 \nu_{k}\right]}{\Gamma\left[2 \nu_{k}\right]} \frac{\Gamma\left[\frac{1}{2}+\nu_{k}-i q e_{d}\right]}{\Gamma\left[\frac{1}{2}-\nu_{k}-i q e_{d}\right]} \frac{\Gamma\left[\frac{1}{2}+\nu_{k}+i q e_{d}-i \omega \zeta_{0}\right]}{\Gamma\left[\frac{1}{2}-\nu_{k}+i q e_{d}-i \omega \zeta_{0}\right]} .
$$

By using that finite temperature definition $T$ as in Eq. (C101), we have

$$
\mathcal{G}^{T}(\omega, k)=(4 \pi T)^{2 \nu_{k}} \frac{\Gamma\left[-2 \nu_{k}\right]}{\Gamma\left[2 \nu_{k}\right]} \frac{\Gamma\left[\frac{1}{2}+\nu_{k}-i q e_{d}\right]}{\Gamma\left[\frac{1}{2}-\nu_{k}-i q e_{d}\right]} \frac{\Gamma\left[\frac{1}{2}+\nu_{k}+i q e_{d}-i \frac{\omega}{2 \pi T}\right]}{\Gamma\left[\frac{1}{2}-\nu_{k}+i q e_{d}-i \frac{\omega}{2 \pi T}\right]} \equiv T^{2 \nu_{k}} g_{b}\left(\nu_{k}, \frac{\omega}{2 \pi T}\right),
$$

where $g_{b}$ is a scaling function given by $[75,76]$ 


$$
\begin{aligned}
g_{b}\left(\nu_{k}, x\right) \equiv & (4 \pi)^{2 \nu_{k}} \frac{\Gamma\left[-2 \nu_{k}\right]}{\Gamma\left[2 \nu_{k}\right]} \frac{\Gamma\left[\frac{1}{2}+\nu_{k}-i q e_{d}\right]}{\Gamma\left[\frac{1}{2}-\nu_{k}-i q e_{d}\right]} \\
& \times \frac{\Gamma\left[\frac{1}{2}+\nu_{k}+i q e_{d}-i x\right]}{\Gamma\left[\frac{1}{2}-\nu_{k}+i q e_{d}-i x\right]} .
\end{aligned}
$$

It is worthy of noticing that at zero temperature the original branch point at $\omega=0$ disappears and the branch cut is replaced at finite temperature by a line of poles parallel to the next imaginary axis. In the zero temperature limit $(T \rightarrow 0)$, these pole line emerges as a branch cut. At finite low temperature, the near horizon geometry is a black brane in $\mathrm{AdS}_{2}$. This IR geometry results in the Green's functions at the finite temperature. The fermion self-energy at finite temperature becomes

$$
\begin{array}{r}
\Sigma(\omega, T)=T^{2 \nu_{k}} g_{b}\left(\frac{\omega}{T}\right) \sim(4 \pi T)^{2 \nu_{k}} \frac{\Gamma\left[\frac{1}{2}+\nu_{k}+i q e_{d}-i \frac{\omega}{2 \pi T}\right]}{\Gamma\left[\frac{1}{2}-\nu_{k}+i q e_{d}-i \frac{\omega}{2 \pi T}\right]} \\
\stackrel{T \rightarrow 0}{\rightarrow}(4 \pi T)^{2 \nu_{k}}\left(-i \frac{\omega}{2 \pi T}\right)^{2 \nu_{k}} \sim c_{k} \omega^{2 \nu_{k} .} \quad(\mathrm{C} 118)
\end{array}
$$

In the zero temperature limit, i.e., $T \rightarrow 0$, the line of discrete poles of the Gamma function at finite temperature emerges as a branch cut for $\omega^{2 \nu_{k}}$ at $T=0$.

Last but not the least, the finite temperature metric in Eq. (C100) is equivalent to a zero temperature Poincare $\mathrm{AdS}_{2}$ metric as

$$
d s^{2}=\frac{\ell_{2}^{2}}{\sigma^{2}}\left(-d \tau^{2}+d \sigma^{2}\right),
$$

through a coordinate transformation as

$$
\tau \pm \sigma=\zeta_{0} e^{\frac{t}{\zeta_{0}} \operatorname{tarctan} \frac{\zeta}{\zeta_{0}}}
$$

At the $\mathrm{AdS}_{2}$ boundary, one has

$$
\tau=\frac{1}{2 \pi T} e^{2 \pi T t},
$$

which is the transformation that generates a uniform accelerating Rindler space in the $t$ coordinate from the vacuum in the $\tau$ coordinate. This is related to the Euruh effect, which states that the background becomes warm in an accelerating reference frame. An unifromly accelerating observer will observe the ground state of the initial observer as one in thermodynamic equilibrium with a finite temperature.

\section{e. Disordered state:quantum liquid phase}

At the moment, let's discuss the physical properties of the boundary field theory in the IR, namely $\mathrm{CFT}_{1}$, or $(0+1)$-dimensional conformal quantum mechanics. The physics is totally characterized by the dimension of the operator $\Phi$ at the IR fixed point, which should be matched by the operator of the boundary field theory $\mathcal{O}$ from the bulk scalar field $\phi(t, x, z)$. Thus, the retarded function of $\phi_{k}$ at the IR fixed point can be written as in Eq. (C87)

$$
\begin{aligned}
\mathcal{G}_{k}(\omega) & =c\left(\nu_{k}\right)(i \omega)^{2 \nu_{k}}, \\
c\left(\nu_{k}\right) & =2^{2 \nu_{k}} \frac{\Gamma\left[-2 \nu_{k}\right]}{\Gamma\left[2 \nu_{k}\right]} \frac{\Gamma\left[\frac{1}{2}-i q e_{d}+\nu_{k}\right]}{\Gamma\left[\frac{1}{2}-i q e_{d}-\nu_{k}\right]},
\end{aligned}
$$

where conformal index $\nu_{k}$ is given in Eq. (C57), which can be reexpressed as

$$
\begin{aligned}
d \geq 2: \nu_{k} & =\sqrt{\frac{1}{4}+\left(m^{2}+\frac{k^{2} \ell^{2}}{r_{\star}^{2}}-\frac{q^{2} e_{d}^{2}}{\ell_{2}^{2}}\right) \ell_{2}^{2}}, \\
d & =1: \nu_{1}=\sqrt{\frac{1}{4}+m^{2}-\mu^{2}} .
\end{aligned}
$$

As the physical consequences, the boundary operators with larger momentum $k$ become less relevant in the IR, while the boundary operator with larger $q$ will have more significant IR fluctuations. In the infinite $\mathrm{AdS}_{2}$ boundary, the electric field linearly blows up and becomes strong field $A_{t}(\zeta)=e_{d} / \zeta^{\zeta \rightarrow 0} \infty$. The spectrum weight scales with $\omega$ as a power for any momentum $|k|$

$$
\operatorname{Im} \mathcal{G}_{k}(\omega)=(-1)^{\nu_{k}} \operatorname{Im}\left[c\left(\nu_{k}\right)\right] \omega^{2 \nu_{k}} \sim \omega^{\frac{\ell|k|}{\sqrt{d(d-1)} r_{\star}}}
$$

which indicates that the presence of low-energy excitation for all momenta (including those at larger momenta, although it will more suppressed due to larger scaling dimension). The conformal index $\nu_{k}$ can be rewritten as

$$
\begin{aligned}
\nu_{k} & =\frac{1}{\sqrt{d(d-1)}} \sqrt{\frac{d(d-1)}{4}+\left(m^{2}+\frac{k^{2}}{r_{\star}^{2}}-2 q^{2}\right) \ell^{2}} \\
& \equiv \frac{1}{\sqrt{d(d-1)}} \frac{\ell^{2}}{r_{\star}} \sqrt{\frac{1}{\xi^{2}}+k^{2},} \quad \nu_{k=0}=\frac{1}{\sqrt{d(d-1)}} \frac{\ell^{2}}{r_{\star}} \frac{1}{\xi},
\end{aligned}
$$

where $\nu_{k}$ has a branch point at $k=i \xi^{-1}$, and $\xi$

$$
\xi \equiv\left[\sqrt{\frac{d(d-1)}{4}+\left(m^{2}-\frac{q^{2} e_{d}^{2}}{\ell_{2}^{2}}\right) \ell^{2}}\right]^{-1} \frac{\ell^{2}}{r_{\star}},
$$

is the correlation length. By using the definition that $r_{\star} \equiv$ $\ell^{2} / z_{\star}$ in energy coordinate, as defined as $z_{\star}=\ell^{2} / r_{\star}=$ $\sqrt{d(d-1)} g_{F} / \sqrt{2}(d-2) \mu$. the dimension index and correlation length can be expresses as 


$$
\begin{aligned}
\nu_{k} & =\frac{z_{\star}}{\sqrt{d(d-1)}} \sqrt{\frac{1}{\xi^{2}}+k^{2}} \\
\xi & =\frac{z_{\star}}{\sqrt{d(d-1)}}\left[\sqrt{\frac{1}{4}+\left(m^{2} \ell_{2}^{2}-q^{2} e_{d}^{2}\right)}\right]^{-1} .
\end{aligned}
$$

In the limit that $|\vec{x}| \ll \xi,|\vec{k}| \ll \xi^{-1}, \nu_{k} \approx \nu_{k=0}$, one only need to focus on the time component of the Fourier transform from $\omega$ to $t$, the retarded function of $\phi_{k}$ at the IR fixed point is

$$
\mathcal{G}^{R}(t, k) \sim \frac{1}{t^{1+2 \nu_{k}}} \approx \frac{1}{t^{2 \Delta_{k=0}}} \sim t^{-1-\frac{1}{\sqrt{d(d-1)} t^{2} r_{\xi}}}
$$

In the limit that $|\vec{x}| \gg \xi,|\vec{k}| \gg \xi^{-1}$, one only needs to focus on the spatial component of the Fourier transform from $\vec{k}$ to $\vec{x}$, the correlation function decays at least exponentially as

$\mathcal{G}_{E}^{R}(t, x) \sim e^{-k_{0}|\vec{x}|}=e^{-\frac{|\vec{x}|}{\zeta}} \sim e^{-\frac{(d-2)}{\sqrt{2}} \sqrt{\frac{1}{4}+m^{2} \ell_{2}^{2}-q^{2} e_{d}^{2}} \mu|\vec{x}|}$,

where $E$ indicates it is in Euclidean spacetime. Intuitively speaking, the system are separated into independent domains of size of order $\xi$, according to Eq. (C129), domains separated by distances larger than $\xi$ are uncorrelated with one another. Within each of the domain, the dynamics of the domain are controlled by $\mathrm{CFT}_{1}$, namely a conformal quantum mechanics in the time direction with a power law correlation, according to the Eq. (C128). Given the system has a nonzero entropy density, each cluster has a nonzero entropy that counts the number of degrees of freedom inside the domain.

To be brief, the correlation functions and the scaling dimension index in Eq. (C122) describes a disordered state, or a quantum liquid phase, where the space factorizes into independent domains of correlation length $\xi$. Within each domain, one has scale invariance along the time direction. However, it is worthy of emphasizing that the scaling behavior within each cluster here describes not the behavior of a single site, but the collective behavior of a large number of sites over a size of order $\xi$. The correlation function $\mathcal{G}_{k}$ and scaling dimension index $\nu_{k}$ depend nontrivially on $k$. Generally speaking, a generic point in parameter space the dependence of $\nu_{k}$ and $\mathcal{G}_{k}$ on $k$ is analytic and only through $k / z_{\star} \sim k r_{\star}$. Near certain quantum critical points (QCP), the dependence of $\nu_{k}$ and $\mathcal{G}_{k}$ on $k$ at $k=0$ becomes nonanalytic.

\section{Spectral asymmetry}

For finite temperature case, Eq. (C116) can be reexpressed as [55]

$$
\begin{aligned}
\mathcal{G}^{R}(\omega, T)= & \mathcal{G}^{A *}(\omega, T) \\
= & \frac{1}{(4 \pi T)^{1-2 \Delta}} \frac{\Gamma[1-2 \Delta]}{\Gamma[2 \Delta-1]} \frac{\Gamma\left[\Delta-i q e_{d}\right]}{\Gamma\left[1-\Delta-i q e_{d}\right]} \\
& \times \frac{\Gamma\left[\Delta+i q e_{d}-i \frac{\omega}{2 \pi T}\right]}{\Gamma\left[1-\Delta+i q e_{d}-i \frac{\omega}{2 \pi T}\right]} \\
= & \frac{-i C e^{-i \theta}}{(\beta / 2 \pi)^{2 \Delta-1}} \frac{\Gamma\left[\Delta-i \frac{\beta}{2 \pi}\left(\omega-\omega_{s}\right)\right]}{\Gamma\left[1-\Delta-i \frac{\beta}{2 \pi}\left(\omega-\omega_{s}\right)\right]},
\end{aligned}
$$

where $\Delta=\nu_{k}+1 / 2$, and we have removed the subscript index $k$ for $\mathcal{G}_{k}^{R, A}$, which is not $k$ dependent explicitly. $c=$ $|c(\Delta-1 / 2)|$ is a real constant with $c\left(\nu_{k}\right)$ as defined in Eq. (C92)

$$
\begin{aligned}
c\left(\nu_{k}\right) & =\left|c\left(\nu_{k}\right)\right| e^{i \theta_{k}}=-i C e^{-i \theta} \\
& =\frac{1}{2^{1-2 \Delta}} \frac{\Gamma[1-2 \Delta]}{\Gamma[2 \Delta-1]} \frac{\Gamma\left[\Delta-i q e_{d}\right]}{\Gamma\left[1-\Delta-i q e_{d}\right]},
\end{aligned}
$$

and the phase $\theta_{k}=-\pi / 2-\theta$. Generally speaking, the phase $\theta_{k}$ is not independent of the momentum $k$ in high dimensional spacetime. In the above deduction, we have used Eqs. (C95) and (C96). It depends upon the normalization basis one has chosen as in Eq. (C109).

The fermionic retarded Green's function has a same form as the Bosonic one, except that the scaling dimension becomes $\Delta_{ \pm}=1 / 2 \pm \nu_{k}$ with $\nu_{k}=\sqrt{m_{k}^{2} \ell_{2}^{2}-q^{2} e_{d}^{2}}$ [56], and Eq. (C95) becomes [35,41,55]

$$
e^{2 \pi q e_{d}}=\frac{\sin (\pi \Delta+\theta)}{\sin (\pi \Delta-\theta)}=e^{\omega_{s} \beta},
$$

where in the second equality we have used Eq. (C96) for finite temperature case and $\theta \in(-\pi \Delta, \pi \Delta)$ for fermion. A nonvanishing $e_{d}$, thus $\omega_{s}$ characterize the spectral asymmetry when the particle-hole symmetry is not kept [53]. It is useful to express the numerator and denominator as

$\sin (\pi \Delta \pm \theta)=\frac{\sin (2 \pi \Delta) e^{ \pm \pi q e_{d}}}{\sqrt{2} \sqrt{\cos (2 \pi \Delta)+\cosh \left(2 \pi q e_{d}\right)}}$,

and the spectral asymmetry angle $\theta$ can also be reexpressed in terms of $e_{d}$ given $\Delta$ as

$$
\theta=\arctan \left[\tan (\pi \Delta) \tanh \left(\pi q e_{d}\right)\right] .
$$

The phase $\theta=0$ corresponds to the particle-hole symmetry. In this case, one just obtains $\sin (\pi \Delta)=\sin (2 \pi \Delta) /$ $\sqrt{2(1+\cos (2 \pi \Delta))}$. The spectral asymmetry angle as a function of $e_{d}$ and $\Delta$ are plotted in Fig. 15.

The universal quantity $e_{d}$ plays a role of spectral asymmetry which can be calculated as [77]

$$
2 \pi e_{d} \equiv \frac{d s}{d n}=-\left(\frac{\partial \mu}{\partial T}\right)_{n}
$$




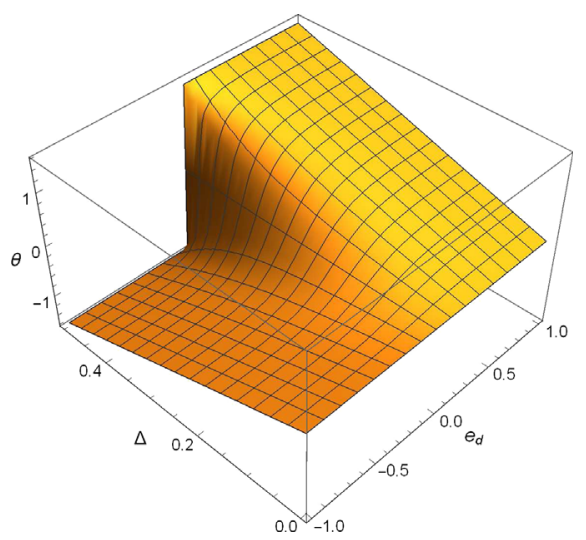

(a) $\theta\left(e_{d}, \Delta\right)$

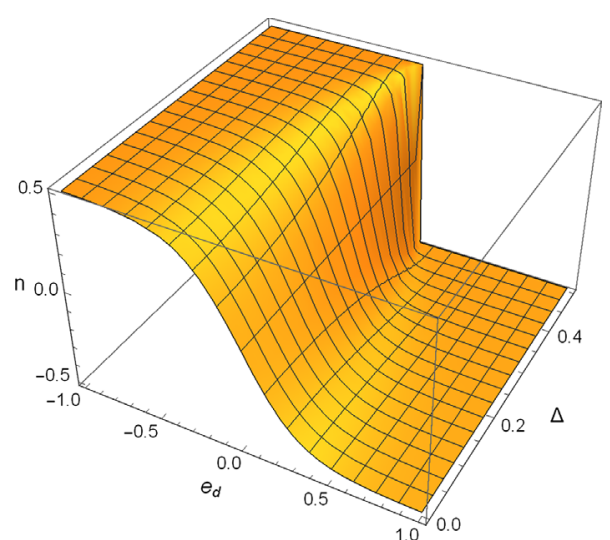

(b) $n\left(e_{d}, \Delta\right)$

FIG. 15. Spectral asymmetric angle $\theta$ and number density $n$ vs effective IR charge $e_{d}$ and conformal scaling dimension $\Delta$ : (a) $\theta\left(e_{d}, \Delta\right)$; (b) $n(n, \Delta)$. Where $\theta$ is given in Eq. (C134) and $n$ is given in (C136). For $\Delta=1 / 2$, the number density $n$ has a typical Fermi liquid like behavior at zero temperature, since it becomes as a step function of $e_{d}$. The spectral asymmetric angle also has a similar behavior at $\Delta=1 / 2$ but is vanishing when $\Delta=0$.

where $s$ is the entropy density, $n$ is the particle number density defined as $n \equiv n_{0}-1 / 2 \in(-1 / 2,1 / 2)$ with the average fermion number $n_{0} \equiv N^{-1} \sum_{i} \chi_{i}^{\dagger} \chi_{i}$ is proportional to the two-point correlator as $[16,41,53]$

$$
\begin{aligned}
n_{0} & =\mathcal{G}^{F}\left(\tau=0^{-}\right) \equiv \frac{1}{\beta} \sum_{n} \mathcal{G}^{F}\left(i \omega_{n}\right) e^{i \omega_{n} 0^{+}}=\lim _{t \rightarrow 0^{+}} i \int_{-\infty}^{\infty} \frac{d \omega}{2 \pi} e^{i(\omega+i \epsilon) t} \mathcal{G}^{F}(\omega)=-\frac{1}{\pi} \int_{-\infty}^{+\infty} d \omega \rho(\omega) n_{F}(\omega) \\
& =\frac{1}{2}-\frac{\theta}{\pi}+\left(\Delta-\frac{1}{2}\right) \frac{\sin (2 \theta)}{\sin (2 \pi \Delta)}=\frac{1}{2}-\frac{\arctan \left(\tan (\pi \Delta) \tanh \left(\pi q e_{d}\right)\right)}{\pi}+\frac{(2 \Delta-1) \sinh \left(2 \pi q e_{d}\right)}{2\left(\cos (2 \pi \Delta)+\cosh \left(2 \pi q e_{d}\right)\right)},
\end{aligned}
$$

where $n_{0} \in(0,1), \theta \in(-\pi / 4, \pi / 4), \mathcal{G}^{F}$ is the Feynman Green's function on the real axis. In the last equality, we have used Eq. (C134). Therefore, the value of spectral asymmetry angle $\theta$ can be related to the particle number density $n$. The density number $n$ as a function of $e_{d}$ and $\Delta$ is plotted in Fig. 15. $\mu$ is the chemical potential $\mu \equiv(\partial F / \partial n)_{T}$ is defined through the free energy given temperature fixed. For $\mathrm{SYK}_{4}$ case $(\Delta=1 / 4), \mathrm{SYK}_{2}(\Delta=1 / 2)$ and $\mathrm{SYK}_{p=\infty}$, respectively, one has

$$
n_{0} \stackrel{\Delta=1 / 4}{=} \frac{1}{2}-\frac{\arctan \left(\tanh \left(\pi q e_{d}\right)\right)}{\pi}-\frac{1}{4} \tanh \left(2 \pi q e_{d}\right), \quad n_{0} \stackrel{\Delta=1 / 2}{=} 1, \quad n_{0} \stackrel{\Delta=0}{=}-\frac{1}{2} \tanh \left(\pi q e_{d}\right) .
$$

For RN-AdS ${ }_{d+1}$ case, $e_{d}$ is just the IR effective electric field in $\mathrm{AdS}_{2}$ region. The $e_{d}$ is independent of the temperature $\beta$ provided $n$ is held fixed. The particle number density $n_{c}$ is a monotonically decreasing function from 1 to 0 as $q e_{d}$ is increasing from $-\infty$ to $+\infty$. Intuitively,

$$
\begin{aligned}
q e_{d} & =(-\infty, 0,+\infty), \quad \Leftrightarrow n_{0}=\left(1, \frac{1}{2}, 0\right), \\
n & =\left(\frac{1}{2}, 0,-\frac{1}{2}\right),
\end{aligned}
$$

which corresponds to the cases that the band is full filling, half filling and empty, respectively. The half-filling case $\left(n_{0}=1 / 2\right)$ corresponds to the particle-hole symmetric case [53].

\section{a. Zero temperature case}

At zero temperature limit, one just recovers Eq. (C87), which plays a role as an ansatz for the form of the low frequency IR conformal invariant retarded Green's function at finite chemical potential as

$$
\begin{aligned}
\mathcal{G}^{R}(\omega) & =\mathcal{G}^{A \star}(\omega)=|c(k)| e^{i \gamma_{k}} \omega^{2 \nu_{k}}=(-i)^{2 \nu_{k}}\left|c\left(\nu_{k}\right)\right| e^{i \theta_{k}} \omega^{2 \nu_{k}} \\
& =-i C e^{-i \theta}\left(-i\left(\omega-\omega_{s}\right)\right)^{2 \Delta-1}=C \frac{e^{-i(\pi \Delta+\theta)}}{\left(\omega-\omega_{s}\right)^{1-2 \Delta}},
\end{aligned}
$$

where $C$ is a real constant to be determined selfconsistently in SD equation, for SYK model as shown in Eq. (C172). $\Delta$ is the fermion scaling dimension, $\operatorname{Im}(\omega)>0$ and the complex frequency $|\omega| \ll J . \theta$ is the spectrum asymmetry phase determined in Eq. (C134). For $\mathcal{G}_{k}^{R} \sim|\omega|^{2 \nu_{k}}$, the inverse Fourier transformation gives the result $-\sqrt{2 / \pi}|t|^{-1-2 \nu_{k}} \Gamma\left[1+2 \nu_{k}\right] \sin \left(\pi \nu_{k}\right) \sim t^{-2 \Delta}$. This implies a coordinate space correlation function by doing an inverse Fourier transformation, 


$$
\mathcal{G}^{R}(t)=-\frac{i \sqrt{\frac{\pi}{2}} C(|t|+t) e^{-i\left(\omega_{s}|t|+\theta\right)}}{|t|^{2 \Delta} t \Gamma(1-2 \Delta)} \sim \frac{1}{t^{2 \Delta}}
$$

with the conformal dimension $\Delta$ of the bosonic operator $\mathcal{O}_{B}$ given by $\Delta=1 / 2+\nu_{k}$. By doing transformation to the Euclidean time coordinate, i.e., $t=i \tau$, the retarded Green's function becomes

$$
\begin{aligned}
\mathcal{G}^{R}(\tau) & =-\frac{\sqrt{\frac{\pi}{2}} C(|\tau|+i \tau)|\tau|^{-2 \Delta} e^{-i\left(\omega_{s}|\tau|+\theta\right)}}{\tau \Gamma(1-2 \Delta)} \\
& =\frac{1}{\beta} \sum_{i \omega_{n}} \mathcal{G}^{E}\left(i \omega_{n}\right) e^{-i \omega_{n} \tau} \\
& =-\frac{1}{2 \pi i} \int_{C} d \omega n_{F}(\omega) \mathcal{G}^{E}\left(i \omega_{n}\right) e^{-i \omega_{n} \tau},
\end{aligned}
$$

where $n_{F}=\left(e^{\beta \omega}+1\right)^{-1}=[1-\tanh (\beta \omega / 2)] / 2$ is the Fermi-Dirac distribution and $\mathcal{G}^{E}\left(i \omega_{n}\right)$ is the Green's function at the Matsubara frequencies, which can be analytically continue to Lorentzian signature inside the integral as

$$
\mathcal{G}^{E}\left(i \omega_{n}\right)=\int_{0}^{\beta} d \tau e^{i \omega_{n} \tau} \mathcal{G}(\tau)
$$

where $\omega_{n}=2 \pi(n+1 / 2) / \beta$ is Matsubara frequency for fermion (For bosonic case, they are $\omega_{n}=2 \pi n / \beta$ ) and the retarded and advanced Green's function can be expressed, respectively, as

$$
\begin{aligned}
& \mathcal{G}^{R}(\omega)=\mathcal{G}^{E}\left(i \omega_{n}=\omega+i \epsilon\right)=\mathcal{G}^{A \star}(\omega), \\
& \mathcal{G}^{A}(\omega)=\mathcal{G}^{E}\left(i \omega_{n}=\omega-i \epsilon\right),
\end{aligned}
$$

where $\epsilon$ is a positive infinitesimal. The fermionic Green's function is defined as $\mathcal{G}(\tau)=-\left\langle T_{\tau} \chi(\tau) \chi^{\dagger}(0)\right\rangle$ in imaginary time $\tau$ coordinate (For bosonics case, it is defined as $\left.\mathcal{G}(\tau)=\left\langle T_{\tau} \phi(\tau) \phi^{\dagger}(0)\right\rangle\right)$, and it can be continued to all complex frequencies by taking a Hilbert transform in the spectral representation as

$$
\mathcal{G}(\omega)=\int_{-\infty}^{+\infty} \frac{d \omega^{\prime}}{\pi} \frac{\rho\left(\omega^{\prime}\right)}{\omega-\omega^{\prime}},
$$

and the retarded and advanced Green's functions turns out to be $\mathcal{G}^{R, A}=\mathcal{G}(\omega \pm i \epsilon)$, respectively, where $\rho(\omega)$ is the zero temperature spectral function defined as imaginary part of retarded Green's function

$$
\begin{aligned}
\rho(\omega) \equiv & \operatorname{Im} \mathcal{G}^{R}(\omega) \stackrel{ \pm \omega>0}{=}-C \sin ( \pm \pi \Delta+\theta) \\
& \times\left( \pm\left(\omega-\omega_{s}\right)\right)^{2 \Delta-1}>0, \quad \pm \operatorname{Re} \omega>0
\end{aligned}
$$

where we have used Eq. (C139).

The two-point correlation function can be obtained by using spectral representation as [53]

$$
\mathcal{G}(\tau)=\frac{1}{\pi} \int_{-\infty}^{+\infty} d \omega \frac{\rho(\omega) e^{-\omega \tau}}{1+e^{-\beta \omega}}, \quad \pm \tau>0, \quad \tau \in[0, \beta]
$$

According to the sign of $\tau$, after imposing the inverse Fourier transform, one obtains the zero temperature $(\beta=\infty)$ Green's function as below

$$
\begin{aligned}
\mathcal{G}( \pm \tau) & \stackrel{ \pm \tau>0}{=} \pm \int_{0}^{\infty} \frac{d \omega}{\pi} \rho( \pm \omega) e^{\mp \omega \tau}=\mp \frac{C}{\pi| \pm \tau|^{2 \Delta}} \sin (\pi \Delta \pm \theta) e^{\mp \omega_{s} \tau} \Gamma\left(2 \Delta, \mp \tau \omega_{s}\right) \\
& =\mp \frac{C}{\pi| \pm \tau|^{2 \Delta}} \sin (\pi \Delta \pm \theta) e^{\mp \frac{2 \pi}{\beta} q e_{d} \tau} \Gamma\left(2 \Delta, \mp \frac{2 \pi}{\beta} q e_{d} \tau\right), \quad \pm \tau \gg J^{-1}>0, \quad-\operatorname{Re}\left(\omega_{s}\right)>0,
\end{aligned}
$$

where $\theta \in(-\pi \Delta, \pi \Delta)$ (for boson case, $\theta \in(\pi \Delta$, $\pi(1-\Delta)))$ and he particle-hole symmetry is exact at $\theta=0$, namely, $\Delta=0$, or $p=\infty . \Gamma(z, a) \equiv \int_{a}^{\infty} t^{z-1} e^{-t} d t$ is the incomplete gamma function, and $\Gamma(z, 0)=\Gamma(z)$ only in the $z>0$ range, since the former has a branch cut discontinuity in the complex $z$-plane running from $-\infty$ to 0 , while the later has no branch cut discontinuities.

In the asymmetric case $\left(\omega_{s} \neq 0\right)$, the retarded Green's function can be estimated as

$$
(\mathcal{G}(+\tau), \mathcal{G}(-\tau)) \stackrel{ \pm \tau>0}{\sim} C \frac{\left(1, e^{2 \pi q e_{d}}\right)}{|\tau|^{2 \Delta}}
$$

where we have used the definition of spectral asymmetry $e_{d}$ in terms of the phase of fermionic retarded Green's function in Eq. (C132).

In the spectral density symmetric case $\left(\omega_{s}=0\right)$, the zero temperature retarded Green's function can be rewritten as [55]

$$
\mathcal{G}( \pm \tau)=\mp \frac{C}{\pi|\tau|^{2 \Delta}} \sin (\pi \Delta \pm \theta) \Gamma(2 \Delta),
$$

where $\pm \tau \gg J^{-1}>0, \mp \operatorname{Re}\left(\omega_{s}\right)>0, \Delta>0$.

In the small asymmetric case $\left(\omega_{s} \ll 1\right)$, the zero temperature retarded Green's function can be expanded as 


$$
\begin{aligned}
\mathcal{G}( \pm \tau)= & \mp \frac{C}{\pi} \sin (\pi \Delta \pm \theta)\left[\frac{\Gamma(2 \Delta)}{| \pm \tau|^{2 \Delta}}\left(1 \mp \tau \omega_{s}+\frac{\left(\omega_{s} \tau\right)^{2}}{2} \mp \frac{\left(\omega_{s} \tau\right)^{3}}{3 !}+\frac{\left(\omega_{s} \tau\right)^{4}}{4 !}+\cdots\right)\right. \\
& \left.-\omega_{s}^{2 \Delta} e^{-2 \pi \Delta i}\left(\frac{1}{2 \Delta} \mp \frac{\tau \omega_{s}}{2 \Delta(2 \Delta+1)}+\frac{\tau^{2} \omega_{s}^{2}}{2 \Delta(2 \Delta+1)(2 \Delta+2)} \mp+O\left(\left(\tau \omega_{s}\right)^{3}\right)\right)\right] .
\end{aligned}
$$

In the large asymmetric case $\left(\omega_{s} \gg 1\right)$, the zero retarded Green's function behaviors as

$$
\mathcal{G}( \pm \tau) \stackrel{T \rightarrow \infty}{=} \mp \frac{C}{\pi(\mp)^{2 \Delta}} \sin (\pi \Delta \pm \theta) \omega_{s}^{2 \Delta}\left(\mp \frac{1}{\tau \omega_{s}}+\frac{2 \Delta-1}{\tau^{2} \omega_{s}^{2}} \mp \frac{(2 \Delta-1)(\Delta-1)}{\tau^{3} \omega_{s}^{3}}+\mathcal{O}\left(\frac{1}{\tau^{4} \omega_{s}^{4}}\right)\right) .
$$

For $\mathrm{SYK}_{2}$ case $(p=2, \Delta=1 / 2)$, one has

$$
\mathcal{G}( \pm \tau) \stackrel{ \pm \tau>0}{=}-\frac{C}{\pi \tau} \cos (\theta), \quad \pm \tau \gg J^{-1}>0
$$

For $\mathrm{SYK}_{4}$ case $(p=4, \Delta=1 / 4)$, one has

$$
\begin{aligned}
\mathcal{G}( \pm \tau)= & \mp \frac{C}{\pi \sqrt{ \pm \tau}} \sin \left(\frac{\pi}{4} \pm \theta\right) e^{\mp \omega_{s} \tau} \operatorname{erfc}\left(\sqrt{\mp \tau \omega_{s}}\right), \\
& \pm \tau \gg J^{-1}>0
\end{aligned}
$$

where we have used $\Gamma(1 / 2, z)=\sqrt{z} E_{1 / 2}(z)=\sqrt{\pi} \operatorname{erfc}(\sqrt{z})$, where $E_{1 / 2}(z)$ is the exponential integral function
$E_{n}(z)=\int_{1}^{\infty} t^{-n} e^{-z t} d t, \operatorname{erfc}(z)$ is the complementary error function $\operatorname{erfc}(z) \equiv 1-\operatorname{erf}(z)$ defined through the error function as $\operatorname{erf}(z)=2 \sqrt{\pi}^{-1} \int_{0}^{z} e^{-t^{2}} d t$.

In the infinite $p$ limit $(p \rightarrow \infty$ or $\Delta=0)$, the Green's function becomes

$$
\mathcal{G}( \pm \tau) \stackrel{p \gg \infty}{=}-\frac{C}{\pi} \sin \theta e^{\mp \omega_{s} \tau}\left[\Gamma\left(0, \mp \omega_{s} \tau\right)+2 \Delta\left(G_{2,3}^{3,0}\left(\mp \tau \omega_{s} \mid \begin{array}{c}
1,1 \\
0,0,0
\end{array}\right) \pm\left[\pi \cot \theta \pm 2 \ln \left(-\omega_{s}\right)\right] \Gamma\left(0, \mp \omega_{s} \tau\right)\right)+\mathcal{O}\left(\Delta^{2}\right)\right]
$$

where $E_{i}(z)=-\int_{-z}^{+\infty} t^{-1} e^{-t} d t$ is the exponential integral function.

In the large $p$ limit (small $\Delta$ limit), one has

$$
\mathcal{G}( \pm \tau) \stackrel{p \rightarrow \infty}{=} \frac{C}{\pi} \sin (\theta) e^{\mp \omega_{s} \tau} E_{i}\left( \pm \omega_{s} \tau\right)
$$

where $G_{2,3}^{3,0}$ is the Meijer-G function defined in Eq. (4.14).

For $\mathrm{SYK}_{p}$ model, Eq. (C139) is the IR ansatz at complex frequency for the Green's function and effective action is

$$
\bar{S}=-\ln \operatorname{Det}\left[\partial_{\tau}+\mu-\Sigma(\tau)\right]+\mu \beta+\int_{0}^{\beta} d \tau\left(\Sigma(\tau) \mathcal{G}(-\tau)+(-1)^{p / 2} J^{2}[\mathcal{G}(\tau) \mathcal{G}(-\tau)]^{\frac{p}{2}}\right)
$$

where $\ln \operatorname{Det}[\cdots]=\operatorname{Tr} \ln [\cdots]$. The last term is just the Luttinger-Ward functional $[53,77] \Phi_{L W} \equiv \int_{0}^{\beta} d \tau[\mathcal{G}(\tau) \mathcal{G}(-\tau)]^{p / 2}$. By doing variation with respect to $\mathcal{G}(-\tau)=-\mathcal{G}(\beta-\tau)$ from and the self-energy $\Sigma(\tau)$, respectively, one just obtains the selfenergy and SD equation of generalized SYK model at large $\mathrm{N}$ saddle point as [55]

$$
\Sigma(\tau)=\frac{\delta \Phi_{L W}}{\delta \mathcal{G}(-\tau)}=-(-1)^{p / 2} p J^{2}[\mathcal{G}(\tau)]^{p / 2}[\mathcal{G}(-\tau)]^{p / 2-1}, \quad \mathcal{G}_{0}^{-1}(\omega+i \epsilon)-\Sigma(\omega+i \epsilon)=\mathcal{G}^{-1}(\omega+i \epsilon)
$$

where $p=\Delta^{-1}, \mathcal{G}_{0}(\omega)=(\omega+i \epsilon-\mu)^{-1}$ is the Green's function for noninteracting fermions and we have make the replacement $i \omega_{n}=\omega+i \epsilon$ for the freely interacting Green's functions without interactions at the Matsubara frequency is

$$
\mathcal{G}_{0}^{-1}\left(i \omega_{n}\right)=-i \omega_{n}+\mu .
$$

At the IR limit $(\omega \rightarrow 0)$, the kinetic term $\partial_{\tau}$ is irrelevant, the SD equation are invariant under the time-reparametrization. For $\mathrm{SYK}_{4}, \mathrm{SYK}_{2}$ and $\mathrm{SYK}_{1}$, according to Eq. (C157), the self-energy can be respective represented in terms of correlation functions, respectively, as $\Sigma(\tau)=-4 J^{2} \mathcal{G}(\tau)^{2} \mathcal{G}(-\tau), \Sigma(\tau)=2 J^{2} \mathcal{G}(\tau), \Sigma(\tau)=-i J^{2}$. For $p=1$ case, the self-energy is a constant, thus, the correlation function is $\mathcal{G}(\omega+i \epsilon)^{-1}=\omega+i \epsilon-\mu+i J^{2}$. The decay width or inverse decay time is 
$\Gamma=-2 \operatorname{Im} \Sigma(\tau)=2 J^{2}$, thus in the strongly coupling limit $(J \rightarrow \infty)$, the quasiparticle is unstable and will decay rapidly in $\mathrm{SYK}_{1}$ case $(p=1)$. For $\mathrm{SYK}_{2}$ case $(p=2)$ case, it is straightforward to solve the self-consistent SD equation to obtain [54]

$$
\Sigma(\omega)=\frac{\omega+i \epsilon+\mu}{2} \pm \sqrt{\left(\frac{\omega+i \epsilon+\mu}{2}\right)^{2}-2 J^{2}}=\frac{\omega+\mu}{2} \pm i \sqrt{2 J^{2}-\left(\frac{\omega+\mu}{2}\right)^{2}} .
$$

Thus, the DOS of $\mathrm{SYK}_{2}$ turns out to be a semicircle centered at $\mu$ as

$$
\rho(\omega) \equiv \operatorname{Im} \mathcal{G}(\omega)=\frac{1}{2 J^{2}} \operatorname{Im} \Sigma(\omega)=\frac{1}{2 J^{2}} \sqrt{2 J^{2}-\left(\frac{\omega+\mu}{2}\right)^{2}} \theta\left(J-\frac{|\omega+\mu|}{2 \sqrt{2}}\right) .
$$

Therefore, for $p=2$ case, the Green's function has non-Fermi liquid like behavior, e.g., there is no quasiparticle pole. For $\mathrm{SYK}_{4}$, one may also obtain its self-energy in the strong coupling limit as

$$
\Sigma(\omega) \stackrel{J \rightarrow \infty}{=} \sqrt{2 J}+\frac{\omega+\mu}{8 J}-\frac{(\omega+\mu)^{2}}{128 \sqrt{2} J^{5 / 2}}+\mathcal{O}\left(J^{11 / 2}\right) .
$$

In the following, we will mainly focus on $p \geq 2$ case.

At zero temperature, by substituting the zero temperature Green's function ansatz in Eq. (C147) into the gap equation in Eq. (C157), one obtains zero temperature self-energy as

$$
\begin{aligned}
\Sigma( \pm \tau) & =-p J^{2}[-\mathcal{G}( \pm \tau)]^{p / 2}[\mathcal{G}(\mp \tau)]^{p / 2-1}=\frac{p J^{2}}{-\mathcal{G}(\mp \tau)}[-\mathcal{G}(\tau) \mathcal{G}(-\tau)]^{p / 2} \\
& =\mp p J^{2} \frac{C^{p-1}}{\pi^{p-1}} \frac{|\mp \tau|^{2 \Delta}}{|+\tau|^{p \Delta}|-\tau|^{p \Delta}} e^{\mp \omega_{s} \tau} \frac{\left[\Gamma\left(2 \Delta,-\tau \omega_{s}\right) \Gamma\left(2 \Delta,+\tau \omega_{s}\right)\right]^{p / 2}}{\Gamma\left(2 \Delta, \pm \tau \omega_{s}\right)} \frac{[\sin (\pi \Delta+\theta) \sin (\pi \Delta-\theta)]^{p / 2}}{\sin (\pi \Delta \mp \theta)} \\
& =\mp p J^{2}\left(\frac{C}{\pi|\tau|^{2 \Delta}}\right)^{p-1} e^{\mp \omega_{s} \tau} \frac{\left[\Gamma\left(2 \Delta,-\tau \omega_{s}\right) \Gamma\left(2 \Delta,+\tau \omega_{s}\right)\right]^{p / 2}}{\Gamma\left(2 \Delta, \pm \tau \omega_{s}\right)} \frac{[\sin (\pi \Delta+\theta) \sin (\pi \Delta-\theta)]^{p / 2}}{\sin (\pi \Delta \mp \theta)},
\end{aligned}
$$

where we have used the zero temperature Green's function ansatz in Eq. (C147), and also $(-1)^{p / 2}=e^{-i \pi / 2}$.

For particle-hole symmetric case $\left(\omega_{s}=0\right)$, the self-energy function becomes

$$
\Sigma( \pm \tau)=\mp p J^{2}\left(\frac{C \Gamma(2 \Delta)}{\pi}\right)^{p-1} \frac{|\mp \tau|^{(2-p) \Delta}}{| \pm \tau|^{p \Delta}} \frac{[\sin (\pi \Delta+\theta) \sin (\pi \Delta-\theta)]^{p / 2}}{\sin (\pi \Delta \mp \theta)} .
$$

After doing Fourier transformation, one has

$$
\begin{aligned}
\Sigma(\omega) & \stackrel{ \pm \omega>0}{=} \int_{0}^{\beta \rightarrow \infty} d \tau \Sigma( \pm \tau) e^{\mp \omega \tau} \\
& =-\left.p J^{2}\left(\frac{C \Gamma(2 \Delta)}{\pi}\right)^{p-1} \Gamma(1-2 \Delta(p-1), x)\right|_{x= \pm \beta \omega} ^{x=0} \frac{[\sin (\pi \Delta+\theta) \sin (\pi \Delta-\theta)]^{p / 2}}{\sin (\pi \Delta \mp \theta)} \omega^{2 \Delta(p-1)-1},
\end{aligned}
$$

and by using $\Gamma(a,+\infty)=0$, and Euler's reflection formula $\Gamma(1-x) \Gamma(x)=\pi / \sin (\pi x)$, one obtains

$$
\Sigma(\omega)=-\frac{\pi p J^{2}}{\Gamma(2(p-1) \Delta)}\left(\frac{C \Gamma(2 \Delta)}{\pi}\right)^{p-1} \frac{[\sin (\pi \Delta+\theta) \sin (\pi \Delta-\theta)]^{p / 2}}{\sin (2 \Delta(p-1) \pi) \sin (\pi \Delta \mp \theta)} \omega^{2 \Delta(p-1)-1} .
$$

By using Eq. (C134), the zero temperature self-energy can be reexpressed as

$$
\Sigma(\tau)=p J^{2}(-C)^{p-1}\left(\frac{\sin (2 \pi \Delta)}{\sqrt{2} \sqrt{\cos (2 \pi \Delta)+\cosh \left(2 \pi q e_{d}\right)}}\right)^{p-1} \frac{e^{\pi q e_{d}}}{\tau^{2 \Delta(p-1)}}\left(\frac{\Gamma(2 \Delta)}{\pi}\right)^{p-1} \sim \frac{1}{\tau^{2 \Delta_{\Sigma}}},
$$

where $C$ is determined in Eq. (C172) with $p=1 / \Delta$. By comparing with the $\mathcal{G}(\tau)$ in Eq. (C149), one may make a new notation $\Delta_{\Sigma} \equiv \Delta(p-1)=1-\Delta$ as the conformal scaling dimension for the self-energy. 
Similarly, the self-energy $\Sigma(\tau)$ can be transformed to the $\Sigma\left(i \omega_{n}\right)$ through

$\Sigma(\tau)=\frac{1}{\beta} \sum_{i \omega} \Sigma\left(i \omega_{n}\right) e^{-i \omega_{n} \tau}, \quad \Rightarrow \Sigma\left(i \omega_{n}\right)=\int_{0}^{\beta} d \tau \Sigma(\tau) e^{i \omega_{n} \tau}$.

$(\mathrm{C} 167)$

In the frequency spacetime, one has the imaginary part of the self-energy, $\Gamma(\omega)=-\operatorname{Im}(\Sigma(\omega))=-\Sigma^{\prime \prime}(\omega)>0$, namely, the decay width or inverse life time as

$$
\begin{aligned}
\Sigma(\omega) \stackrel{ \pm \omega>0}{\equiv} & \pm \int_{0}^{\beta \rightarrow \infty} \Sigma( \pm \tau) e^{\mp \omega \tau}=-J^{2} p\left(\frac{C \Gamma(2 \Delta)}{\pi}\right)^{p-1} \\
& \times \frac{\pi}{\Gamma(2 \Delta(p-1))} \frac{[\sin (\pi \Delta-\theta) \sin (\pi \Delta+\theta)]^{p / 2}}{\sin (\pi \Delta(p-1) x) \sin (\pi \Delta \mp \theta)} \\
& \times( \pm \omega)^{2 \Delta(p-1)-1},
\end{aligned}
$$

where we have $\operatorname{sgn}(\operatorname{Im} \Sigma(\omega+i \epsilon))=\operatorname{sgn}\left(\Sigma^{\prime \prime}(\omega+i \epsilon)\right)=$ $-\operatorname{sgn}(\omega+i \epsilon)$, assumed $\operatorname{Re} \Sigma(0)=\Sigma^{\prime}(0)=\mu$ at $T=0$. The self-energy $\Sigma(\omega)$ can be expressed in the spectral representation with the spectral function $\sigma(\omega) \equiv$ $\operatorname{Im} \Sigma(\omega)=\Sigma^{\prime \prime}(\omega)$ as

$$
\Sigma(\omega)=\int_{-\infty}^{+\infty} \frac{d \omega^{\prime}}{\pi} \frac{\sigma\left(\omega^{\prime}\right)}{\omega-\omega^{\prime}} .
$$

The gap equations should be satisfy also at the IR limit (the kinetic term $\partial_{\tau}$ is absent), i.e., the interacting Green's function at Matsubara frequency is

$$
\mathcal{G}\left(i \omega_{n}\right)^{-1}=-i \omega_{n}+\mu-\Sigma\left(i \omega_{n}\right),
$$

which leads to $\mathcal{G}(\omega+i \epsilon)^{-1}=\omega+\mu-\Sigma(\omega+i \epsilon)+i \epsilon$, namely, by using Eq. (C139) with $\omega_{s}=0$, it should be $C^{-1} e^{+i(\pi \Delta+\theta)} \omega^{1-2 \Delta}=-(\Sigma(\omega)-\mu)+i \epsilon=-[i \operatorname{Im} \Sigma(\omega)+$ $\operatorname{Re} \Sigma(\omega)-\mu]$ with $\mu=\operatorname{Re} \Sigma(0)$, and at the low temperature $(\omega \ll \Sigma(\omega)$, with $(\omega \gg \Sigma(\omega)$ as the Fermi liquid, since the interaction becomes), in which limit, the SYK model has an asymptotic exact infrared time reparametrization symmetry under $\tau \rightarrow f(\tau)$. By comparing with the imaginary parts in both sides, one has

$$
p=\frac{1}{\Delta}, \quad-J^{2} p C^{p}\left(\frac{\Gamma(2 \Delta)}{\pi}\right)^{p-1} \Gamma(1-2 \Delta(p-1))[\sin (\pi \Delta-\theta) \sin (\pi \Delta+\theta)]^{\frac{p}{2}-1}=1,
$$

from which the constant $C$ can be determined as

$$
\begin{gathered}
C^{-p}=-\frac{\pi p J^{2}}{\Gamma(2 \Delta(p-1)) \sin (2 \pi(p-1) \Delta)}\left(\frac{\Gamma(2 \Delta)}{\pi}\right)^{p-1}[\sin (\pi \Delta-\theta) \sin (\pi \Delta+\theta)]^{\frac{p}{2}-1} \\
\stackrel{p=\Delta^{-1}}{=} \frac{\pi J^{2}}{\Delta \Gamma(2(1-\Delta)) \sin (2 \pi \Delta)}\left(\frac{\Gamma(2 \Delta)}{\pi}\right)^{\frac{1}{\Delta}-1}\left(\frac{\sin (2 \pi \Delta)}{\sqrt{2} \sqrt{\cos (2 \pi \Delta)+\cosh \left(2 \pi q e_{d}\right)}}\right)^{\frac{1}{\Delta}-2},
\end{gathered}
$$

where we have used Euler's reflection formula as $\Gamma(-x)=-\pi[\Gamma(x+1) \sin (\pi x)]^{-1}$ and in the last equality, we substitute back the phase constant $\theta$ in Eq. (C133).

The constants $C$ and $\theta$, or equivalently the density asymmetry $e_{d}$, can be determined exactly for the microscopic models, which depends upon the UV completion of the theory, which is not universal. For the original SYK 4 model case ( $p=4$ and $\Delta=1 / 4)$, the constant $C$ turns out to be

$$
C^{-4}=\frac{4 \pi J^{2}}{\Gamma\left(\frac{3}{2}\right)}\left(\frac{\pi}{\Gamma\left(\frac{1}{2}\right)}\right)^{3} \frac{1}{2} \cos 2 \theta, \quad \Rightarrow C=\left(\frac{\pi}{4 J^{2} \cos (2 \theta)}\right)^{\frac{1}{4}}=\left(\frac{\pi \cosh \left(2 \pi q e_{d}\right)}{4 J^{2}}\right)^{\frac{1}{4}} .
$$

For the $\mathrm{SYK}_{2}$ model $(p=2$ and $\Delta=1 / 2)$, the constant $C$ turns out to be $C=1 / \sqrt{2 J^{2}}$.

\section{b. Finite temperature case}

The finite temperature solutions for $\mathcal{G}^{T}(\omega)$ and $\Sigma^{T}(\omega)$ can be obtained by using the fact that an IR time reparametrization symmetry is asymptotically exact as in Eq. (A8). One can map $t \rightarrow f(\tau)$, i.e., from a line $t \in$ $(-\infty,+\infty)$ at $T=0$ to $\tau \in(0, \beta)$ at $T \neq 0$ as

$$
t \rightarrow f(\tau)=\frac{1}{2} \tan \frac{t}{2} \rightarrow \frac{\beta}{\pi} \tan \frac{\pi}{\beta} \tau
$$

where we have restored the common factor in Eq. (2.39) by rescaling $t \rightarrow 2 \pi / \beta t . \tau$ is the periodic imaginary time coordinate with period $\beta$. Since $f^{\prime}(\tau)=\sec ^{2}(\pi \tau / \beta)$, the emergent conformal symmetry, or timereparametrization symmetry in Eq. (A8) can be expressed more explicitly as 
$\mathcal{G} \rightarrow\left|\sec (\pi \tau / \beta) \sec \left(\pi \tau^{\prime} / \beta\right)\right|^{2 \Delta} \mathcal{G}\left(f(\tau), f\left(\tau^{\prime}\right)\right)$,

$\Sigma \rightarrow\left|\sec (\pi \tau / \beta) \sec \left(\pi \tau^{\prime} / \beta\right)\right|^{2(1-\Delta)} \Sigma\left(f(\tau), f\left(\tau^{\prime}\right)\right)$,

where we also used the constraint $p=1 / \Delta$ in Eq. (C171) for SYK model.

The finite temperature retarded Green's function can be obtained from Eq. (C149) with symmetric density of spectrum through the conformal transformation above as

$$
\begin{aligned}
\mathcal{G}^{T}( \pm \tau)= & \mp C g(\tau)\left(\frac{1}{\frac{\beta}{\pi} \sin \left( \pm \frac{\pi}{\beta} \tau\right)}\right)^{2 \Delta} \sin (\pi \Delta \pm \theta) \frac{\Gamma(2 \Delta)}{\pi} \\
& \pm \tau \in(0, \beta)
\end{aligned}
$$

where $g(\tau)$ is a function with normalization condition $g(0)=1$. By imposing the Kubo-Martin-Schwinger (KMS) boundary condition $\mathcal{G}^{T}(\tau+\beta)=-\mathcal{G}^{T}(\tau)$, one obtains

$$
g(\tau+\beta) \frac{\sin (\pi \Delta+\theta)}{\sin (\pi \Delta-\theta)}=g(\tau) .
$$

By using Eq. (C132) again, one has

$$
g(\tau+\beta) e^{2 \pi q e_{d}}=g(\tau) \Rightarrow g(\tau)=e^{-2 \pi q e_{d} \frac{\tau}{\beta}} .
$$

Therefore, Eq. (C176) can be expressed more explicitly as

$$
\mathcal{G}^{T}( \pm \tau)=\mp C \frac{\sin (2 \pi \Delta) e^{ \pm \pi q e_{d}}}{\sqrt{2} \sqrt{\cos (2 \pi \Delta)+\cosh \left(2 \pi q e_{d}\right)}} e^{-2 \pi q e_{d \frac{\tau}{\beta}}}\left(\frac{1}{\frac{\beta}{\pi} \sin \left( \pm \frac{\pi}{\beta} \tau\right)}\right)^{2 \Delta} \frac{\Gamma(2 \Delta)}{\pi} \sim \frac{1}{J^{\frac{2}{p}}}, \quad \pm \tau \in(0, \beta)
$$

and its Fourier transformation leads to the Eq. (C130). This implies that there is a frequency shift $\omega_{s} \beta$ arising from the nontrivial source of the gauge field as

$$
\omega_{s} \equiv \frac{2 \pi q e_{d}}{\beta}
$$

which turns out to be self-consistent with Eq. (C96). In the zero temperature limit $(\beta \rightarrow \infty)$, Eq. (C179) becomes

$$
\mathcal{G}^{T}( \pm \tau)=\mp C \frac{\sin (2 \pi \Delta) e^{ \pm \pi q e_{d}}}{\sqrt{2} \sqrt{\cos (2 \pi \Delta)+\cosh \left(2 \pi q e_{d}\right)}} \frac{\pi^{-2 \Delta}}{( \pm \tau)^{2 \Delta}} \frac{\Gamma(2 \Delta)}{\pi}, \quad \pm \tau \in(0, \beta)
$$

For original SYK model $(\Delta=1 / 4)$, one just recovers

$$
\begin{aligned}
\mathcal{G}^{T}( \pm \tau) & =\mp C \frac{e^{-2 \pi q e_{d} \frac{\tau}{\beta}}}{\sqrt{1+e^{\mp 4 \pi q e_{d}}}}\left(\frac{1}{\frac{\beta}{\pi} \sin \left( \pm \frac{\pi}{\beta} \tau\right)}\right)^{\frac{1}{2}} \frac{1}{\sqrt{\pi}} \sim \frac{1}{J^{\frac{1}{2}}} \\
& =\mp\left(\frac{\left[\pi \cosh \left(2 \pi q e_{d}\right)\right]^{\frac{1}{4}}}{\sqrt{2} J^{1 / 2}}\right) \frac{e^{-2 \pi q e_{d}^{\frac{\tau}{\beta}}}}{\sqrt{1+e^{\mp 4 \pi q e_{d}}}}\left(\frac{1}{\beta \sin \left( \pm \frac{\pi}{\beta} \tau\right)}\right)^{\frac{1}{2}}, \quad \pm \tau \in[0, \beta)
\end{aligned}
$$

where we have used Eq. (C172). In the zero temperature limit, one has

$$
\mathcal{G}^{T}( \pm \tau)=\mp\left(\frac{\left[\pi \cosh \left(2 \pi q e_{d}\right)\right]^{\frac{1}{4}}}{\sqrt{2} J^{1 / 2}}\right) \frac{1}{\sqrt{1+e^{\mp 4 \pi q e_{d}}}} \frac{1}{| \pm \tau|^{1 / 2}}, \quad \pm \tau \in[0, \beta) .
$$

At finite temperature case, the self-energy, according to Eq. (C157), and by using KMS relation $\mathcal{G}^{T}(-\tau)=-\mathcal{G}^{T}(\beta-\tau)$, it turns out to be

$$
\begin{aligned}
\Sigma(\tau) & =p J^{2}\left[\mathcal{G}^{T}(\tau)\right]^{p / 2}\left[\mathcal{G}^{T}(\beta-\tau)\right]^{p / 2-1} \\
& =p J^{2}(-C)^{p-1}\left(\frac{\sin (2 \pi \Delta)}{\sqrt{2} \sqrt{\cos (2 \pi \Delta)+\cosh \left(2 \pi q e_{d}\right)}}\right)^{p-1}\left(\frac{1}{\frac{\beta}{\pi} \sin \left(\frac{\pi}{\beta} \tau\right)}\right)^{2 \Delta(p-1)} e^{\pi q e_{d}} e^{-2 \pi q e_{d} \frac{\tau}{\beta}}\left(\frac{\Gamma(2 \Delta)}{\pi}\right)^{p-1} \\
& =\frac{1}{\Delta} J^{2}(-1)^{\frac{1}{\Delta}-1}\left(\frac{\pi J^{2}}{\Delta(1-2 \Delta)}\right)^{\Delta-1}\left(\frac{\sin (2 \pi \Delta)}{\sqrt{2} \sqrt{\cos (2 \pi \Delta)+\cosh \left(2 \pi q e_{d}\right)}}\right)^{2(1-\Delta)}\left(\frac{1}{\frac{\beta}{\pi} \sin \left(\frac{\pi}{\beta} \tau\right)}\right)^{2(1-\Delta)} e^{\pi q e_{d}} e^{-2 \pi q e_{d} \frac{\tau}{\beta}} .
\end{aligned}
$$

If there is no spectral density asymmetry $\left(e_{d}=0\right)$, 


$$
\sigma(\tau)=\frac{1}{\Delta} J^{2 \Delta}(-1)^{\frac{1}{\Delta}-1}\left(\frac{\Delta(1-2 \Delta)}{\pi} \frac{\tan ^{2}(\pi \Delta)}{2}\right)^{(1-\Delta)}\left(\frac{1}{\frac{\beta}{\pi} \sin \left(\frac{\pi}{\beta} \tau\right)}\right)^{2(1-\Delta)}
$$

By doing a Fourier transformation, after changing from the imaginary time to the real time coordinate, i.e., $\tau=-i t$, one obtains the finite temperature self-energy with density asymmetry as

$$
\begin{aligned}
\Sigma(\omega)= & \int_{0}^{\infty} d \tau \Sigma(\tau) e^{-\omega \tau}=-i \int_{0}^{\infty} d t \Sigma(-i t) e^{i \omega t}=-i \frac{1}{\Delta} J^{2}(-1)^{\frac{1}{\Delta}-1}\left(\frac{\pi J^{2}}{\Delta(1-2 \Delta)}\right)^{\Delta-1} \frac{1}{(-2 i)^{2(1-\Delta)}} \\
& \times\left(\frac{\sin (2 \pi \Delta)}{\sqrt{2} \sqrt{\cos (2 \pi \Delta)+\cosh \left(2 \pi q e_{d}\right)}}\right)^{2(1-\Delta)} \int_{0}^{\infty} d t e^{i \omega t} e^{\pi q e_{d}} e^{+2 \pi q e_{d} \frac{t}{\sigma^{i}}}\left(\frac{1}{\frac{\beta}{2 \pi} \sinh \left(\frac{\pi}{\beta} t\right)}\right)^{2(1-\Delta)} \\
= & i \frac{\beta}{2 \pi} \frac{1}{\Delta} J^{2 \Delta}(-1)^{\frac{1}{\Delta}}\left(\frac{\Delta(1-2 \Delta)}{-8 \pi} \frac{\sin ^{2}(2 \pi \Delta)}{\cos (2 \pi \Delta)+\cosh \left(2 \pi q e_{d}\right)}\right)^{1-\Delta} e^{\pi q e_{d}} I_{-\Delta+1-i \frac{\beta}{2 \pi}\left(\omega-\omega_{s}\right)}^{0},
\end{aligned}
$$

where $\omega_{s}=2 \pi q e_{d} / \beta$ with $\beta>0$ and $\operatorname{Im} \omega>0$, we have used Eq. (D11), the $I_{\nu}^{0}$ is defined in Eq. (D9). The inverse lifetime for the fermions are defined by the $\tau^{-1}=-2 \operatorname{Im} \sigma(0)=-\operatorname{Im} \Sigma(\omega+i \epsilon)$.

Take $\mathrm{SYK}_{4}$ as an example, one has

$$
\Sigma(\omega)=-\frac{\sqrt{J} e^{\pi q e_{d}}(-1)^{3 / 4} \operatorname{sech}^{3 / 4}\left(2 \pi q e_{d}\right)}{\sqrt{2} \pi^{1 / 4}}\left(\frac{4 \pi}{\beta}\right)^{\frac{1}{2}} \frac{\Gamma\left(\frac{3}{4}-\frac{i \beta\left(\omega-\omega_{s}\right)}{2 \pi}\right)}{\Gamma\left(\frac{1}{4}-\frac{i \beta\left(\omega-\omega_{s}\right)}{2 \pi}\right)} \propto \sqrt{J},
$$

where we have used Eq. (D9). For density symmetric case $\left(e_{d}=0\right)$, one has

$$
\Sigma(\omega)=\frac{\sqrt{2 J}}{\pi^{1 / 4}(-1)^{1 / 4}}\left(\frac{\pi}{\beta}\right)^{\frac{1}{2}} \frac{\Gamma\left(\frac{3}{4}-\frac{i \beta \omega}{2 \pi}\right)}{\Gamma\left(\frac{1}{4}-\frac{i \beta \omega}{2 \pi}\right)} \frac{T=0}{=} \frac{\sqrt{2 J}}{\pi^{1 / 4}} \frac{\sqrt{\omega}}{\sqrt{2}},
$$

where the zero temperature self-energy is consistent with Eq. (C168) for $\Delta=1 / 4$ case.

\section{Residual entropy}

In this section, we calculate the $T=0$ residual entropy of the $\mathrm{SYK}_{p}$ model with the density spectral asymmetry, as a generalization to the special result in $\mathrm{SYK}_{4}[1]$.

By substituting back the saddle-point solutions as the self-consistent ones to the SD equation in Eq. (C157) into the effective action in Eq. (C156), one can obtain the full free energy density for the system can be reexpressed as

$$
\bar{F}=\frac{F}{N}=-\ln \operatorname{det}\left[\mathcal{G}^{-1}(\tau)\right]-\left(1-p^{-1}\right) \int_{0}^{\beta} d \tau \Sigma(\tau) \mathcal{G}(-\tau) \equiv \bar{F}_{1}-\left(1-p^{-1}\right) \bar{F}_{2} .
$$

\section{a. Zero temperature case}

For the free energy density $\bar{F}_{1}$ due to the kinetic term of interacting fermion, one has

$$
\bar{F}_{1} \equiv-\operatorname{Tr} \ln \mathcal{G}^{-1}=\operatorname{Tr} \ln \mathcal{G}=\operatorname{Tr} \ln \mathcal{G}_{0}+\operatorname{Tr} \ln \frac{\mathcal{G}}{\mathcal{G}_{0}}
$$

where we have used the standard procedure of adding and substracting the contribution of a free local fermion.

The first part of the $\bar{F}_{1}$ is

$$
\operatorname{Tr} \ln \mathcal{G}_{0}=-\frac{1}{\beta} \sum_{i \omega_{n}} \ln \left(-i \omega_{n}+\mu\right) e^{i \omega_{n} 0^{+}}=\int d \mu \frac{1}{\beta} \sum_{i \omega_{n}} \frac{e^{i \omega_{n} 0^{+}}}{i \omega_{n}-\mu}=\int \frac{d \mu}{1+e^{\beta \mu}}=-\frac{1}{\beta} \ln \left(1+e^{-\beta \mu}\right)^{\mu=0}=-\frac{1}{\beta} \ln 2,
$$

and it is worthy of noticing that it can also be expressed as $\int_{-\infty}^{+\infty} d \omega[\theta(-\omega)-1] n_{F}=\left.\left(\omega+\beta^{-1} \ln n_{F}\right)\right|_{\omega=0}=-\beta^{-1} \ln 2$. 
The second part of $\bar{F}_{1}$ turns out to be

$$
\begin{aligned}
\operatorname{Tr} \ln \frac{\mathcal{G}}{\mathcal{G}_{0}} & =\frac{1}{\beta} \sum_{\omega_{n}} \ln \left[\left(-i \omega_{n}+\mu\right) \mathcal{G}\left(i \omega_{n}\right)\right] e^{i \omega_{n} 0^{+}}=\frac{1}{2 \pi i} \int_{C} d \omega \ln [(\omega-\mu) \mathcal{G}(\omega)] n_{F}(\omega) e^{i \omega_{n} 0^{+}} \\
& =-\int \frac{d \omega^{\prime}}{\pi} \frac{1}{2 \pi i} \int d \omega \ln \left[(\omega-\mu) \frac{\rho\left(\omega^{\prime}\right)}{\omega^{\prime}-\omega}\right] n_{F}(\omega) e^{i \omega_{n} 0^{+}}=-\operatorname{Im} \int \frac{d \omega}{\pi} \ln [(\omega-\mu) \mathcal{G}(\omega)] n_{F}(\omega) \\
& =-\int \frac{d \omega}{\pi} \operatorname{Im} \ln \frac{\mathcal{G}(\omega)}{\mathcal{G}_{0}(\omega)} n_{F}(\omega) e^{i \omega_{n} 0^{+}}=\int \frac{d \omega}{\pi}\left(\arctan \frac{\mathcal{G}^{\prime}(\omega)}{\mathcal{G}^{\prime \prime}(\omega)}+\frac{\pi}{2}-\pi \theta(-\omega)\right) n_{F}(\omega) e^{i \omega_{n} 0^{+}},
\end{aligned}
$$

where we have also used that the identity that $\arctan (\omega)+\arctan (1 / \omega)=\pi / 2-\pi \theta(-\omega) . n_{F}$ is the Fermi-Dirac distribution with $n_{F}(\omega)=\left(e^{\beta \omega}+1\right)^{-1}=-[\tanh (\beta \omega / 2)-1] / 2 . \mathcal{G}=\mathcal{G}^{\prime}+i \mathcal{G}^{\prime \prime}$ and $\mathcal{G}_{0}\left(i \omega_{n}\right)=\left(i \omega_{n}\right)^{-1}$ is the Green function of free electrons after setting $\mu=0$.

Combine the above two contributions together, and one obtains [53]

$$
\begin{aligned}
\bar{F}_{1}=-\frac{1}{\beta} \ln 2+\operatorname{Tr} \ln \frac{\mathcal{G}}{\mathcal{G}_{0}} & =\frac{1}{\pi} \int_{-\infty}^{+\infty} d \omega\left(\arctan \frac{\mathcal{G}^{\prime}(\omega)}{\mathcal{G}^{\prime \prime}(\omega)}-\frac{\pi}{2}\right) n_{F}(\omega) \\
& =\frac{1}{\pi} \int_{0}^{\infty} d \omega n_{F}(\omega)\left[a_{F}(\omega)-a_{F}(-\omega)\right]+\frac{1}{\pi} \int_{-\infty}^{0} d \omega\left[a_{F}(\omega)-a_{F}(-\infty)\right] \\
& \stackrel{e_{d}=0}{=} \frac{2}{\pi} \int_{0}^{\infty} d \omega n_{F}(\omega) a_{F}(\omega)+\frac{1}{\pi} \int_{-\infty}^{0} d \omega\left[a_{F}(\omega)-a_{F}(\omega=-\infty)\right],
\end{aligned}
$$

where we have used $n_{F}(-\omega)=1-n_{F}(\omega)$ and $a_{F}(\omega)$ is defined in Eq. (C195). It is worthy of emphasizing that we have subtracted out a term $a_{F}(-\infty)$ in the integrand, otherwise the integral has a singularity at $\omega=-\infty$. In the last equality, with the assumption that $\theta=0, a_{F}(\omega)=a_{F}(-\omega)$.

For finite temperature correlation functions in Eq. (C130), one obtains

$$
\mathcal{G}(\omega)=\frac{-i C e^{-i \theta}}{\pi(\beta / 2 \pi)^{2 \Delta-1}}\left|\Gamma\left(\Delta-i \frac{\beta}{2 \pi}\left(\omega-\omega_{s}\right)\right)\right|^{2} \sin \left[\pi\left(\Delta+i \frac{\beta}{2 \pi}\left(\omega-\omega_{s}\right)\right)\right],
$$

from which, one obtains $a_{F}$

$$
\begin{aligned}
\tan \left[a_{F}(\omega)\right] \equiv \frac{\mathcal{G}^{\prime}(\omega)}{\mathcal{G}^{\prime \prime}(\omega)} & =\frac{\csc ^{2}(\theta)}{\cot (\pi \Delta) \tanh \left(\frac{1}{2} \beta(\omega-\omega \mathrm{s})\right)+\cot (\theta)}-\cot (\theta) \\
& =\frac{\tan (\pi \Delta) \tanh \left(\pi q e_{d}\right)-\cot (\pi \Delta) \tanh \left(\beta \omega / 2-\pi q e_{d}\right) q e_{d}=0}{1+\tanh \left(\pi q e_{d}\right) \tanh \left(\beta \omega / 2-\pi q e_{d}\right)}-\cot (\pi \Delta) \tanh (\beta \omega / 2)
\end{aligned}
$$

where in the last second equality, we have changed all $\theta$ into $e_{d}$ by using Eq. (C134).

For spectral density symmetric case $\left(e_{d}=0\right)$, since $a_{F}(-\omega)=-a_{F}(\omega)$ the free energy can be expressed as

$$
\begin{aligned}
\bar{F}_{1} & =-\frac{1}{\pi} \int_{-\infty}^{\infty} d \omega \frac{1}{e^{\beta \omega}+1}\left(\arctan [\cot (\pi \Delta) \tanh (\beta \omega / 2)]+\frac{\pi}{2}\right) \\
& =-\frac{2}{\pi} \int_{0}^{\infty} d \omega \frac{1}{e^{\beta \omega}+1} \arctan \left(\frac{\tanh (\beta \omega / 2)}{\tan (\pi \Delta)}\right)-\frac{1}{\pi} \int_{-\infty}^{0} d \omega\left[\arctan \left(\frac{\tanh (\beta \omega / 2)}{\tan (\pi \Delta)}\right)+\arctan \left(\frac{1}{\tan (\pi \Delta)}\right)\right] .
\end{aligned}
$$

According to Eq. (A5), the zero temperature entropy density turns out to be

$$
s_{1}=\beta^{2} \frac{\partial \bar{F}_{1}}{\partial \beta}=\frac{2}{\pi} \int_{0}^{1} d x \frac{\arctan (x / t)}{1+x}+\frac{2}{\pi} \int_{-1}^{0} d x \frac{\arctan (x / t)+\arctan (1 / t)}{1-x^{2}},
$$

where we have denoted $x \equiv \tanh (\omega \beta / 2)$ and $t \equiv \tan (\pi \Delta)$. After finishing the integral, it just recovers the zero temperature entropy of SYK model as $[1,5,6,16]$ 


$$
s_{0}=-\frac{\ln \left(-e^{2 i \pi \Delta}\right)}{\pi i} \ln (2 \cos (\pi \Delta))-\frac{1}{2 \pi i}\left[\operatorname{Li}_{2}\left(-e^{2 \pi i \Delta}\right)-\operatorname{Li}_{2}\left(-e^{-2 \pi i \Delta}\right)\right] .
$$

For $\Delta=0$, it gives $s_{0}=\ln 2=0.693147$, and for $\mathrm{SYK}_{4}$ case $(\Delta=1 / 4)$, it gives $s_{0}=\mathrm{C} / \pi+\ln 2 / 4 \approx 0.464848$, where $\mathrm{C}=\sum_{k=0}^{\infty}(-1)^{k}(2 k+1)^{-2}$ is the Catalan's constant, while for $\mathrm{SYK}_{2}$ case $\Delta=1 / 2$, it gives $s_{0}=0$.

For spectral density asymmetric case $\left(e_{d} \neq 0\right)$, since $a_{F}(-\omega) \neq-a_{F}(\omega)$, the free energy can be expressed as

$$
\begin{aligned}
\bar{F}_{1} & =\frac{1}{\pi} \int_{-\infty}^{\infty} d \omega \frac{1}{e^{\beta \omega}+1}\left[\arctan \left(\frac{\tan (\pi \Delta) \tanh \left(\pi q e_{d}\right)-\cot (\pi \Delta) \tanh \left(\beta \omega / 2-\pi q e_{d}\right)}{1+\tanh \left(\pi q e_{d}\right) \tanh \left(\beta \omega / 2-\pi q e_{d}\right)}\right)-\frac{\pi}{2}\right] \\
& =\int_{0}^{+\infty}\left[n_{F}\left(\omega+\omega_{s}\right) a_{F}\left(\omega+\omega_{s}\right)-n_{F}\left(\omega-\omega_{s}\right) a_{F}\left(-\left(\omega-\omega_{s}\right)\right)\right]+\int_{-\infty}^{0}\left[a_{F}\left(\omega+\omega_{s}\right)-a_{F}(-\infty)\right] .
\end{aligned}
$$

To be more explicitly, by making notations as $x \equiv \tanh (\omega \beta / 2), u \equiv \tanh \left(\pi q e_{d}\right)$ and $t \equiv \tan (\pi \Delta)$, one obtains entropy density $s_{1}$ with an exact analytic formula as

$$
\begin{aligned}
s_{1}= & \int_{0}^{1} \frac{d x}{x+1}\left[\frac{u-1}{u x+1} \arctan \left(\frac{t^{2} u-x}{t u x+t}\right)-\frac{u+1}{u x-1} \arctan \left(\frac{t^{2} u+x}{t-t u x}\right)\right]+\frac{2}{\pi} \int_{-1}^{0} \frac{d x}{x^{2}-1} \arctan \left(\frac{t(1+x)}{x-t^{2}}\right), \\
= & \frac{1}{2}(1-2 \Delta) \ln \left[2\left(\cos (2 \pi \Delta)+\cosh \left(2 \pi q e_{d}\right)\right)\right] \\
& -\frac{i}{4 \pi}\left[\operatorname{Li}_{2}\left(-e^{2 \pi\left(q e_{d}-i \Delta\right)}\right)+\operatorname{Li}_{2}\left(-e^{-2 \pi\left(q e_{d}+i \Delta\right)}\right)-\operatorname{Li}_{2}\left(-e^{2 \pi\left(q e_{d}+i \Delta\right)}\right)-\operatorname{Li}_{2}\left(-e^{2 i \pi \Delta-2 \pi q e_{d}}\right)\right],
\end{aligned}
$$

where in the second integrand, we have used the identity $\arctan (x) \pm \arctan (y)=\arctan [(x \pm y) /(1 \mp x y)]$ to simplify. It is obvious that the second integral is always density symmetric since it is independent of $e_{d}$. It's worthy of noticing that, at the large $p$ limit $(\Delta \rightarrow 0)$, one has

$$
\frac{\partial s_{1}}{\partial \Delta}=\frac{\pi(2 \Delta-1) \sin (2 \pi \Delta)}{\cos (2 \pi \Delta)+\cosh \left(2 \pi q e_{d}\right)}=-\pi(2 \Delta-1) \frac{\cos (2 \pi \Delta)-\cos (2 \theta)}{\sin (2 \pi \Delta)} .
$$

implies that $s_{1}$ has an extremal value at $\theta=\pi \Delta$, combining with $s_{1}^{\prime \prime}(\theta=\pi \Delta) \leq 0$ with the prime denoting the derivative with respect to $\Delta$. Thus, $s_{1}$ achieves its minimum at $\theta=\pi \Delta$. While, for density asymmetric case $\left(e_{d} \neq 0\right)$, the zero temperature entropy will also obtains an extra contribution from the chemical potential $\mu=(\partial \bar{F} / \partial n)_{T}=\mu_{0}-2 \pi q e_{d} T+$ $\cdots$ with $\mu_{0}=\partial e_{0} / \partial n$ from the contribution of zero energy $e_{0}$, it is associated with the particle density $n$ through $\bar{F}_{1}+n \mu$, so that the $T=0$ residual entropy is [53]

$$
s_{0}=s_{1}+\left.n\left(\frac{\partial \mu}{\partial T}\right)\right|_{T=0}=s_{1}-n \frac{\partial s_{1}}{\partial n}=s_{1}+2 \pi q e_{d} n .
$$

where we have used the thermodynamic Maxwell relation in Eq. (C135)

$$
\frac{\partial s}{\partial n}=-\left.\frac{\partial \mu}{\partial T}\right|_{T=0}=-2 \pi q e_{d}=\ln \frac{\sin (\pi \Delta-\theta)}{\sin (\pi \Delta+\theta)} .
$$

For $\Delta=1 / 4$ case, the result recovers Eq. (12) in Ref. [41]. The density $n$ can be obtained through the Maxwell relation in Eq. (C135) as

$$
\begin{aligned}
n=-\frac{1}{2 \pi q} \frac{\partial s_{1}}{\partial e_{d}} & =\frac{(2 \Delta-1) \sinh \left(2 \pi q e_{d}\right)}{2\left(\cos (2 \pi \Delta)+\cosh \left(2 \pi q e_{d}\right)\right)}-\frac{i}{4 \pi}\left[\ln \left(\frac{1+e^{2 \pi\left(q e_{d}-i \Delta\right)}}{1+e^{-2 \pi\left(q e_{d}+i \Delta\right)}}\right)+\ln \left(\frac{1+e^{-2 \pi q e_{d}+2 i \pi \Delta}}{1+e^{2 \pi\left(q e_{d}+i \Delta\right)}}\right)\right] \\
& =\frac{1}{2}(2 \Delta-1) \frac{\sin (2 \theta)}{\sin (2 \pi \Delta)}-\frac{i}{4 \pi} \ln \left(e^{-4 i \theta}\right)
\end{aligned}
$$

where in the last equality, we have used Eq. (C134), and it just recovers the number density given in Eq. (C136). The spectral asymmetry parameter $\theta$ obeys $\theta \in[-\pi \Delta, \pi \Delta]$. 


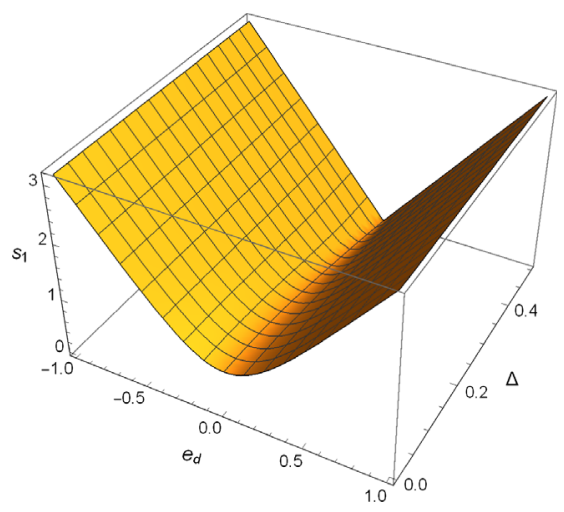

(a) $s_{1}\left(e_{d}, \Delta\right)$

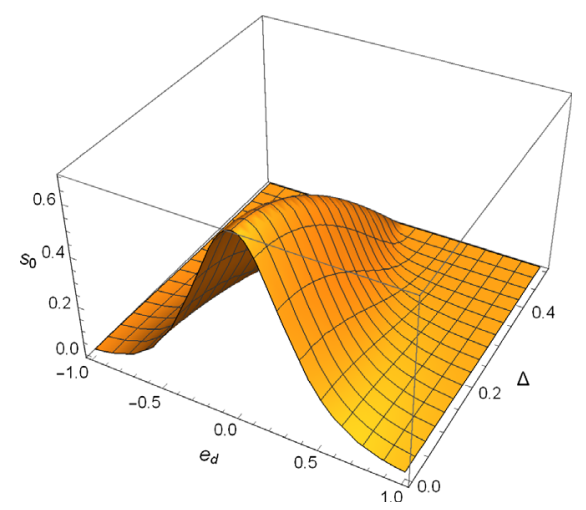

(d) $s_{0}\left(e_{d}, \Delta\right)$

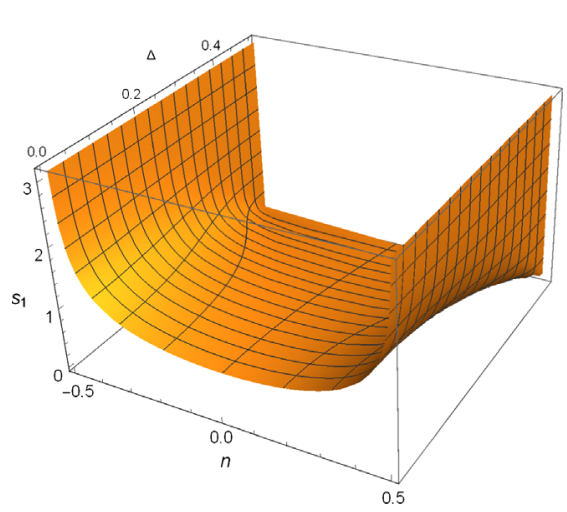

(b) $s_{1}(n, \Delta)$

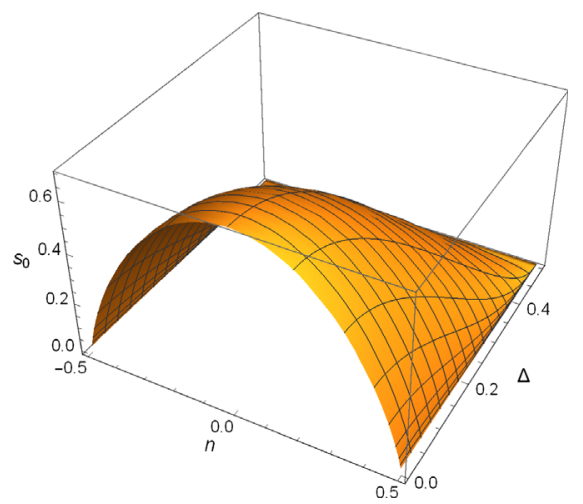

(e) $s_{0}(n, \Delta)$

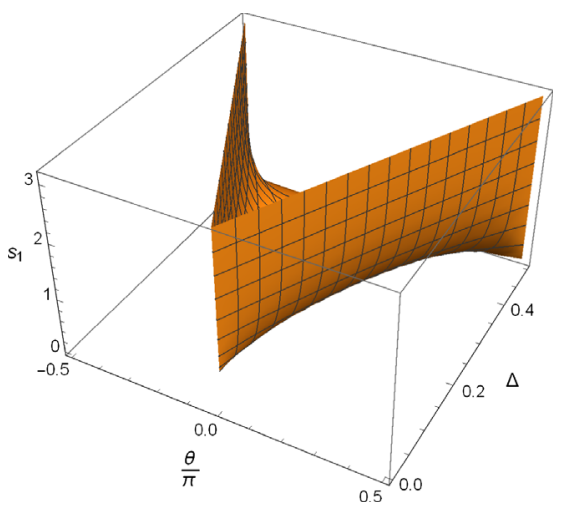

(c) $s_{1}(\theta, \Delta)$

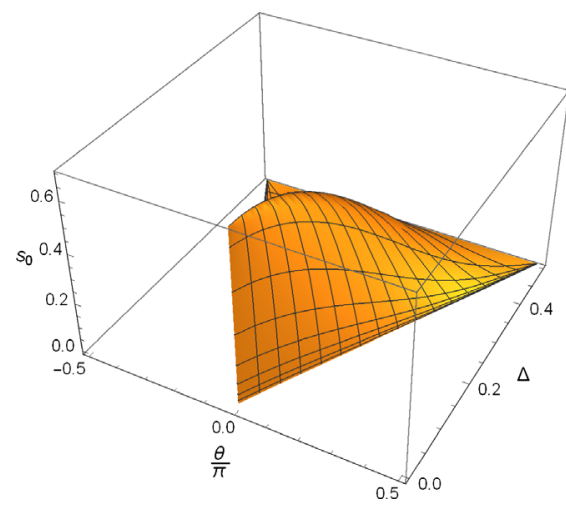

(f) $s_{0}(\theta, \Delta)$

FIG. 16. Residual entropy $s_{0}$ at zero temperature: (a) $s_{1}\left(e_{d}, \Delta\right)$; (b) $s_{1}(n, \Delta)$; (c) $s_{1}(\theta, \Delta)$; (d) $s_{0}\left(e_{d}, \Delta\right)$; (e) $s_{0}(n, \Delta)$; (f) $s_{0}(\theta, \Delta)$. $s_{1}$ is in Eq. (C200) and $s_{0}$ is in Eq. (C202) with the relation $s_{1}=s_{0}$ in the density symmetric case $\left(e_{d}=0\right.$ or $\left.\theta=0, n=0\right)$. $e_{d}$ is the IR effective gauge coupling, $n$ is the number density as in Eq. (C136) or Eq. (C204), $\theta$ is the spectral asymmetry angle in Eq. (C134). We have chosen the parameter $q=1, e_{d} \in[-1 / 2,1 / 2], n \in(0,1), \theta \in[-\pi / 2, \pi / 2]$ and $\Delta \in[0,1 / 2]$.

The $T=0$ residual entropy $s_{0}$ as a function of charge density $e_{d}$, density number $n$, and spectral asymmetric angle $\theta$, respectively, are plotted in Fig. 16, from which, one can observe that

$$
\begin{array}{r}
s_{0}(n=\mp 0.5)=s_{0}\left(e_{d}= \pm 1\right)=0, \\
s_{0}(n=0)=s_{0}\left(e_{d}=0\right)=\ln 2,
\end{array}
$$

which indicates that the zero temperature entropy is vanishing if the band is empty $(n=-0.5)$ or full filling
( $n=0.5$ ), while it achieves its maximum as $\ln 2$, if the band is half filling $(n=0)$, when the particle-hole symmetry is restored $\left(e_{d}=0\right)$. It also indicates the state is one with two different corresponding microstates at zero temperature.

\section{b. Finite temperature case}

For the free energy density $\bar{F}_{2}$ due to self-energy of fermions in Eq. (C189), by using the spectral representation of $\mathcal{G}(-\tau)$ and the definition of $\Sigma(\tau)$, we obtain the second term as [77]

$$
\begin{aligned}
\bar{F}_{2} \equiv \int_{0}^{\beta} d \tau \Sigma(\tau) \mathcal{G}(-\tau) & =\frac{1}{\beta} \sum_{i \omega_{n}} \Sigma\left(i \omega_{n}\right) \mathcal{G}\left(i \omega_{n}\right)=\frac{1}{\beta} \sum_{i \omega_{n}}\left[\mathcal{G}_{0}^{-1}\left(i \omega_{n}\right) \mathcal{G}\left(i \omega_{n}\right)-1\right]=\frac{1}{\beta} \sum_{i \omega_{n}}\left(-i \omega_{n}+\mu\right) \mathcal{G}\left(i \omega_{n}\right) \\
& =-\frac{1}{2 \pi i} \int_{C} d \omega(\mu-\omega) \mathcal{G}(\omega) \frac{1}{2} \tanh \frac{\beta \omega}{2}=\frac{1}{2 \pi i} \int_{C} \frac{d \omega^{\prime}}{\pi} \int d \omega \frac{(\omega-\mu) \rho\left(\omega^{\prime}\right)}{\omega-\omega^{\prime}} n_{F}(\omega),
\end{aligned}
$$

where we have firstly performed a Matsubara sum over discrete imaginary Euclidean frequencies to rewrite the sum over frequencies as a contour integral, secondly used the spectral decomposition in Eq. (C144) and thirdly do an contour integral. Then, the integral can be rearranged as 


$$
\bar{F}_{2}=\int_{-\infty}^{+\infty} \frac{d \omega}{\pi} \frac{(\omega-\mu) \rho(\omega)}{e^{\beta \omega}+1}=\int_{0}^{+\infty} \frac{d \omega}{\pi}[(\omega-\mu) \rho(\omega)+(\omega+\mu) \rho(-\omega)] n_{F}(\omega)+\int_{-\infty}^{0} \frac{d \omega}{\pi}(\omega-\mu) \rho(\omega),
$$

where in the last equality, we have subtracted a term in the integrand so that the integral is not singular at $\omega=-\infty$. Assuming that the spectral density is symmetric $(\rho(\omega)=\rho(-\omega))$, one obtains

$$
\bar{F}_{2}=2 \int_{0}^{+\infty} \frac{d \omega}{\pi} \frac{\omega \rho(\omega)}{1+e^{\beta \omega}}+\int_{-\infty}^{0} \frac{d \omega}{\pi}(\omega-\mu)[\rho(\omega)-\rho(-\infty)] \sim \frac{1}{\beta^{2 \Delta+1}}
$$

where in the last estimation, we have substituted the spectral density $\rho(\omega)$ at finite and zero temperature in Eqs. (C130) and (C145), respectively, into the above formula. One can obtains the finite temperature entropy density, according to the thermodynamics relation in Eq. (A5), as

$$
s_{2}=-\frac{\partial}{\partial T} \bar{F}_{2}=\beta^{2} \frac{\partial \bar{F}_{2}}{\partial \beta} \sim \frac{1}{\beta^{2 \Delta}},
$$

which does not contributes to the zero temperature entropy $s_{0}$ in the density symmetric case $\left(e_{d}=0\right.$ or $\left.\theta=0\right)$.

\section{Conductivity}

For $(0+1)$-dimensional quantum system, the conductivity can be obtained by making perturbation upon the $A_{t}$, i.e., the frequency dependent sector. In generic $\mathrm{RN}-\mathrm{AdS}_{d+1}$ spacetime, to calculate the conductivity on the boundary with the $\mathrm{CFT}_{d}$ symmetry, one needs to study fluctuations of vector gauge fields on the background spacetime. At linear order, the gauge fields satisfy the Maxwell field equations $\partial_{\mu}\left(\sqrt{-g} g^{\mu \rho} g^{\nu \sigma} F_{\rho \sigma}\right)=0$.

Consider turn on both $e^{-i \omega t+i k y} A_{t}(z)$ and $e^{-i \omega t+i k y} A_{y}(z)$ as the perutrbation term, which depends on both frequency and momentum. The linearized Maxwell equation without the metric corrections are the field equation of motion along longitudinal $y$ direction can be generalized to be one in $\operatorname{AdS}_{d+1}$ spacetime as

$$
\begin{aligned}
A_{t}^{\prime \prime}-\frac{d-3}{z} A_{t}^{\prime}-\frac{k^{2}}{f} A_{t}-\frac{k \omega}{f} A_{y} & =0, \\
\omega A_{t}^{\prime}+k f A_{y}^{\prime} & =0, \\
A_{y}^{\prime \prime}+\left(\frac{f^{\prime}}{f}-\frac{d-3}{z}\right) A_{y}^{\prime}+\frac{\omega^{2}}{f^{2}} A_{y}+\frac{k \omega}{f^{2}} A_{t} & =0,
\end{aligned}
$$

where for the RN-AdS ${ }_{d+1}$ case, referring to Eq. (C11), $f=$ $1-\left(z / z_{0}\right)^{d}-Q^{2} z^{d}\left(z_{0}^{d-2}-z^{d-2}\right)$ and $A_{t}=\mu\left(1-\left(z / z_{0}\right)^{d-2}\right)$. The gauge field along the transverse direction, i.e., $x \neq t$, $z, y$ is

$$
A_{x}^{\prime \prime}+\left(\frac{f^{\prime}}{f}-\frac{d-3}{z}\right) A_{x}^{\prime}+\frac{\omega^{2}-k^{2} f}{f^{2}} A_{x}=0 .
$$

Then the longitudinal $A_{y}$ can be solved through

$$
A_{y}^{\prime}=-\frac{i \omega}{\omega^{2}-k^{2} f} E_{y}^{\prime} .
$$

Alternatively, one can combine the first two equations to give the field equations from $A_{t}$ as

$$
A_{t}^{(3)}+\left(\frac{1}{z}+\frac{f^{\prime}}{f}\right) A_{t}^{\prime \prime}+\left(\frac{\left(z f^{\prime}-f\right)}{z^{2} f}+\frac{\omega^{2}-k^{2} f}{f^{2}}\right) A_{t}^{\prime}=0 .
$$

The generic field equation for $A_{t}$ becomes

$$
\begin{aligned}
A_{t}^{(3)} & +\left(\frac{f^{\prime}}{f}-\frac{d-3}{z}\right) A_{t}^{\prime \prime} \\
& +\left[\frac{\omega^{2}-k^{2} f}{f^{2}}-\frac{d-3}{z}\left(\frac{f^{\prime}}{f}-\frac{1}{z}\right)\right] A_{t}^{\prime}=0,
\end{aligned}
$$

and it can be viewed as a second-order equation for $A_{t}^{\prime}$. By making a gauge-invariant combination in terms of electric gauge field $E_{y}=\partial_{y} A_{t}-\partial_{t} A_{y}=i\left(k A_{t}+\omega A_{y}\right)$, the field equations for longitudinal gauge field components $E_{y}$ becomes

$E_{y}^{\prime \prime}+\left(\frac{\omega^{2} f^{\prime}}{\left(\omega^{2}-k^{2} f\right) f}-\frac{d-3}{z}\right) E_{y}^{\prime}+\frac{\omega^{2}-k^{2} f}{f^{2}} E_{y}=0$,

with $f(z)=1-\left(z / z_{0}\right)^{d}$ for pure $\operatorname{AdS}_{d+1}$. One rename $E_{y}=E_{L}$ and $A_{x}=E_{T}$, where $L$ and $T$ denotes the longitudinal and transverse direction, respectively. Then the equations of motion can be reexpressed as

$$
\begin{aligned}
E_{L}^{\prime \prime}+\left(\frac{\omega^{2} f^{\prime}}{\left(\omega^{2}-k^{2} f\right) f}-\frac{d-3}{z}\right) E_{L}^{\prime}+\frac{\omega^{2}-k^{2} f}{f^{2}} E_{L} & =0, \\
E_{T}^{\prime \prime}+\left(\frac{f^{\prime}}{f}-\frac{d-3}{z}\right) E_{T}^{\prime}+\frac{\omega^{2}-k^{2} f}{f^{2}} E_{T} & =0 .
\end{aligned}
$$

It is obvious that for $k=0, E_{L}=E_{T}=E$ satisfy the same equation as 


$$
E^{\prime \prime}+\left(\frac{f^{\prime}}{f}-\frac{d-3}{z}\right) E^{\prime}+\frac{\omega^{2}}{f^{2}} E=0
$$

For $d=1$ case, $k$ is always vanishing. As the physical consequence, one can not distinguish the longitudinal and transversal conductivity. For pure $\mathrm{AdS}_{2}$ case $(d=1)$, one has $f=1-z$, while for $\mathrm{RN}-\mathrm{AdS}_{2}$, one has
$f=\left(1+Q^{2}\right)(1-z)=\left(1+\mu^{2}\right)(1-z)$ and $A_{t}=\mu\left(1-z^{-1}\right)$, where we have set the horizon radius to be $z_{0}=1$. In the following, we will just focus on the pure $\mathrm{AdS}_{2}$ case, the result for the $\mathrm{RN}-\mathrm{AdS}_{2}$ case can be obtained by making a replacement upon the frequency as $\omega \rightarrow \omega /\left(1+\mu^{2}\right)$.

For $\mathrm{AdS}_{2}$ case $(d=1)$ case, the wave function of covariant gauge field $E(z)$ turns out to be

$$
\begin{aligned}
E(z) & =C_{1} e^{\pi \omega}(1-z)^{-i \omega}{ }_{2} F_{1}\left(-i \omega-\sqrt{1-\omega^{2}}+1,-i \omega+\sqrt{1-\omega^{2}}+1 ; 1-2 i \omega ; 1-z\right) \\
& +C_{2} e^{-\pi \omega}(1-z)^{i \omega}{ }_{2} F_{1}\left(i \omega-\sqrt{1-\omega^{2}}+1, i \omega+\sqrt{1-\omega^{2}}+1 ; 2 i \omega+1 ; 1-z\right) \\
& \stackrel{z \rightarrow 1}{\sim} C_{1} e^{\pi \omega}(1-z)^{-i \omega}+C_{2} e^{-\pi \omega}(1-z)^{i \omega},
\end{aligned}
$$

where $e^{-i \omega t}(1-z)^{-i \omega}$ corresponds to the in-falling wave, thus the in-falling boundary condition is $C_{2}=0$. In the UV boundary condition, the asymptotic behavior of the Green's function is

$$
E(z)^{z \rightarrow 0} \stackrel{C_{1} e^{\pi \omega} \Gamma(1-2 i \omega)}{\Gamma\left(1-i \omega-\sqrt{1-\omega^{2}}\right) \Gamma\left(1-i \omega+\sqrt{1-\omega^{2}}\right)}\left(\frac{1}{z}-\ln (z)+1+i \omega-H_{-i \omega-\sqrt{1-\omega^{2}}}-H_{\sqrt{1-\omega^{2}}-i \omega}\right) .
$$

The retarded Green's function turns out to be

$$
\mathcal{G}_{A}(\omega)=\frac{B}{A}=1-2 \gamma_{E}+i \omega-\psi^{(0)}\left(1-\sqrt{1-\omega^{2}}-i \omega\right)-\psi^{(0)}\left(1+\sqrt{1-\omega^{2}}-i \omega\right)+\ln \Lambda,
$$

where the subscript $A$ indicates that it the Green's function for the perturbation of gauge field $\delta A_{\mu}, \ln \Lambda$ is a renormalization dependent contact term and $\psi^{(0)}(z)=\Gamma^{\prime}(z) / \Gamma(z)$. The finite temperature result can be obtained as

$\mathcal{G}_{a}^{T}(\omega)=1-2 \gamma_{E}-i \frac{\omega}{2 \pi T}-\psi^{(0)}\left(-\sqrt{1-\left(\frac{\omega}{2 \pi T}\right)^{2}}-i \frac{\omega}{2 \pi T}\right)-\psi^{(0)}\left(\sqrt{1-\left(\frac{\omega}{2 \pi T}\right)^{2}}-i \frac{\omega}{2 \pi T}\right)+\ln \left(\frac{\Lambda}{2 \pi T}\right)$.

The poles of the Green's function at $-i \omega \pm \sqrt{1-\omega^{2}}=-n$ with $n \in \mathbb{Z}$, results in the frequency modes as

$$
\omega=-\frac{i}{2}\left(n-\frac{1}{n}\right)
$$

which are all in the lower half of the complex plane.

In the zero temperature case, the spectral function of the gauge field in $\operatorname{AdS}_{2}$ is

$$
\begin{aligned}
\rho(\omega)=\operatorname{Im}_{a}(\omega) & =\frac{1}{2} \pi\left(\operatorname{coth}\left[\pi\left(\omega-i \sqrt{1-\omega^{2}}\right)\right]+\operatorname{coth}\left[\pi\left(\omega+i \sqrt{1-\omega^{2}}\right)\right]\right) \\
& =\frac{\pi \sinh (2 \pi \omega)}{\cosh (2 \pi \omega)-\cos \left(2 \pi \sqrt{1-\omega^{2}}\right)}
\end{aligned}
$$

It is worth of noticing that the low frequency behavior of the spectral function is

$$
\rho(\omega) \stackrel{\omega \rightarrow 0}{=} \frac{1}{\omega}+\left(\frac{\pi^{2}}{3}-\frac{1}{4}\right) \omega+\left(-\frac{1}{16}-\frac{\pi^{4}}{45}\right) \omega^{3}+O\left(\omega^{5}\right)
$$

While in the large frequency limit, one has $\lim _{\omega \rightarrow \infty} \rho(\omega) / \omega=1$.

Thus, the conductivity is

$$
\sigma(\omega)=\frac{\mathcal{G}_{a}(\omega)}{i \omega}=\frac{1}{i \omega}\left[1-2 \gamma_{E}-i \omega-\psi^{(0)}\left(-\sqrt{1-\omega^{2}}-i \omega\right)-\psi^{(0)}\left(\sqrt{1-\omega^{2}}-i \omega\right)+\ln \Lambda\right],
$$


where we have used that $\psi^{(0)}(1+i z)=\psi^{(0)}(i z)-i / z$. The low frequency behavior $(\omega \ll T)$ of the conductivity is

$$
\sigma(\omega)=\frac{2 \pi T}{\omega^{2}}-\frac{i}{\omega}\left(\frac{1}{2}+\ln \frac{\Lambda}{2 \pi T}\right)+\frac{4 \pi^{2}-3}{24 \pi T}+\frac{i \omega(4 \zeta(3)-1)}{8 \pi^{2} T^{2}}+O\left(\omega^{2}\right) .
$$

The large frequency behavior $(\omega \gg T)$ of the conductivity is

$$
\sigma(\omega)=\frac{1}{2 \pi T}+\frac{1}{\omega}\left(\frac{\pi}{2}+i\left(\gamma_{E}-1\right)+i \ln \frac{2 \omega}{\Lambda}\right)+\frac{\pi\left(\pi^{2}-3\right)}{6 \omega^{2}} T+O\left(\frac{1}{\omega^{3}}\right) \propto \frac{1}{T} .
$$

\section{a. DC conductivity}

The exact form of the real part of the conductivity, namely, the direct current (DC) conductivity, is

$$
\begin{aligned}
\operatorname{Re}[\sigma(\omega)]= & -\frac{\pi \sinh \left(\frac{\omega}{T}\right)}{\omega \cos \left(2 \pi \sqrt{1-\frac{\omega^{2}}{4 \pi^{2} T^{2}}}\right)-\omega \cosh \left(\frac{\omega}{T}\right)} \stackrel{\omega \rightarrow 0}{=} \frac{2 \pi T}{\omega^{2}} \\
& +\frac{\pi}{6 T}+O(\omega) .
\end{aligned}
$$

\section{b. Optical conductivity}

The imaginary part of the conductivity satisfy the Kramers-Kronig relation, which give the optical conductivity as

$$
\begin{aligned}
\sigma_{o p t}(\omega)= & \operatorname{Im}[\sigma(\omega)]=\int d \omega^{\prime} \frac{\operatorname{Re}\left[\sigma\left(\omega^{\prime}\right)\right]}{\omega-\omega^{\prime}} \stackrel{\omega \rightarrow 0}{=} \\
& -\frac{1}{\omega}\left[\frac{1}{2}+\ln \left(\frac{\Lambda}{2 \pi T}\right)\right]+\frac{\omega(4 \zeta(3)-1)}{8 \pi^{2} T^{2}}+O\left(\omega^{3}\right) .
\end{aligned}
$$

\section{APPENDIX D: SUSCEPTIBILITY}

To appreciate the quantum phase transition with an order parameter, an important observable is susceptibilities, which characterize the dynamical nature of the quantum phase transition. Suppose that the order parameter is given by the expectation value of some bosonic operator $\mathcal{O}$, then the corresponding susceptibility $\chi(\omega, \vec{k})$ is given by the retarded function of $\mathcal{O}$. To be concrete, the uniform/ zero momentum/dynamical susceptibility [38,41] $\chi(\omega)$ and momentum dependent static susceptibility $\chi(\vec{k})$ can be defined, respectively, as

$\chi(\omega) \equiv G^{R}(\omega, \vec{k}=0), \quad \chi(\vec{k}) \equiv G^{R}(\omega=0, \vec{k})$,

and the full dynamical susceptibility $\chi(\omega, \vec{k})$ is defined as

$$
\chi(\omega, \vec{k}) \equiv G^{R}(\omega, \vec{k})
$$

Then, the existence of growing modes with instability are reflected in the singularities of susceptibility in the upper complex momentum space. Thus, it can be indicated by the divergence of uniform static susceptibility $\chi(0)$, The critical behavior of quantum phase transition, is different from the Landau's phase transition where one expects that the uniform susceptibility always diverges when approaching a critical point, e.g., near the critical point, $\chi(0)$ is characterized by a critical exponent $\gamma, \chi(\omega) \sim\left|g-g_{c}\right|^{-\gamma}$ where $g$ is the tuning parameter and $g_{c}$ is the value at critical point. Instead, at the critical point, the uniform static susceptibility remains finite, i.e., $\chi(0) \sim$ const., while the singularity behavior of $\chi(0)$ around $\omega \rightarrow 0$ can be indicated by taking a derivative with respect to $\omega$, thus one finds that $\chi^{\prime}(0)$ is divergent.

In momentum space, the advanced and retarded Green's function as well as the positive/negative Wightman functions are defined as $[53,78]$

$$
\begin{aligned}
& G^{A}(\omega)=i \int_{-\infty}^{+\infty} d t \theta(-t) e^{i \omega t} G(t), \\
& G^{R}(\omega)=-i \int_{-\infty}^{+\infty} d t \theta(t) e^{i \omega t} G(t), \\
& G^{+}(\omega)=\int_{-\infty}^{+\infty} d t e^{i \omega t}\langle\mathcal{O}(t) \mathcal{O}(0)\rangle, \\
& G^{-}(\omega)=\int_{-\infty}^{+\infty} d t e^{i \omega t}\langle\mathcal{O}(0) \mathcal{O}(t)\rangle .
\end{aligned}
$$

Except for the Pauli-Jordan commutator function $G(t) \equiv$ $\langle[\mathcal{O}(t), \mathcal{O}(0)]\rangle$, there are also other real-time two-point functions such as the Hadamard two-point function $G^{H}(t) \equiv\langle\{\mathcal{O}(t), \mathcal{O}(0)\}\rangle$ and the Feynman two-point function $G^{F}(t) \equiv \theta(t)\langle\mathcal{O}(t) \mathcal{O}(0)\rangle+\theta(-t)\langle\mathcal{O}(0) \mathcal{O}(t)\rangle$ can be expressed by linear combinations of the above Green's functions. In momentum space, the finite temperature generalization is Fenman's Green's function as $G_{s}^{F}(\omega, \beta) \equiv$ $\left(1-n_{s}(\omega)\right) G^{R}(\omega)+n_{s}(\omega) G^{R \star}(\omega)$ where $n_{s}(\nu)$ statistic distribution functions, e.g., $s=F$ to denote Fermi-Dirac statistics for fermions, or $s=B$ to denote Bose-Einstein statistics for bosons. In imaginary time, the retarded Green's functions and the Wightman functions are defined as $G^{R}(\tau)=[G(\tau+\epsilon)-G(\tau-\epsilon)] \theta(t)$ and $G^{ \pm}(\tau)=G(\tau \pm \epsilon)$, $G^{ \pm}(t) \stackrel{T \neq 0}{=} G(\tau \pm \beta / 2)$ with $\tau=i t$. The spectral functions of 
quantum liquid is defined as imaginary part of retarded Green's function as

$$
\rho(\omega) \propto-\frac{1}{\pi} \operatorname{Im} G^{R}(\omega) .
$$

The local spin-spin correlation function is directly related to the Green's function and defined as

$$
\chi_{\mathrm{loc}}(\omega) \propto \int_{-\infty}^{+\infty} d t e^{i \omega t} G^{R}(t) G^{R}(-t),
$$

and the local dynamical susceptibility is defined by [38]

$$
\chi_{\mathrm{loc}}^{\prime \prime}(\omega)=\pi \int_{-\infty}^{+\infty} d \nu \rho_{s}(\nu) \rho_{s}(\nu-\omega)\left[n_{s}(\nu-\omega)-n_{s}(\nu)\right],
$$

where $\rho_{s}(\nu)$ is spectral function with spin index $s$ and frequency $\nu$. The static local susceptibility $\chi_{\text {loc }}^{\prime}(\omega=0)$ is defined as

$$
\chi_{\mathrm{loc}}^{\prime}(\omega=0)=\int d \omega \frac{\chi_{\mathrm{loc}}^{\prime \prime}(\omega)}{\omega} .
$$

In dealing with the Fourier transformation for the retarded Green's function with loop corrections, it is useful to introduce a set of integral formulas as follows [53,79]

$$
\begin{aligned}
I_{\Delta-\frac{i \beta \omega}{2 \pi}}^{(0,1,2)} & \equiv \int_{0}^{+\infty} \frac{(2 \pi / \beta) d t e^{i \omega t}}{\left[\frac{\beta}{2 \pi} \sinh \frac{t}{2} \frac{2 \pi}{\beta}\right]^{2 \Delta}}\left\{1, \frac{2 \pi}{\beta} t,\left(\frac{2 \pi}{\beta}\right)^{2} t^{2}\right\} \\
& =I_{\Delta-\frac{i \beta \omega}{2 \pi}}^{(0)}\left\{1,-\psi_{\Delta-i \frac{i \omega}{2 \pi}}^{(0)}, \psi_{\Delta-i \frac{\beta \omega}{2 \pi}}^{(1)}+\left(\psi_{\Delta-i \frac{\beta \omega}{2 \pi}}^{(0)}\right)^{2}\right\}, \\
0 & <\Delta<\frac{1}{2}, \quad \beta>0, \quad \operatorname{Im} \omega>0,
\end{aligned}
$$

where

$$
I_{\Delta-\frac{i \beta \omega}{2 \pi}}^{(0)}=\left(\frac{4 \pi}{\beta}\right)^{2 \Delta} \frac{\Gamma(1-2 \Delta) \Gamma\left(\Delta-\frac{i \beta \omega}{2 \pi}\right)}{\Gamma\left(1-\Delta-\frac{i \beta \omega}{2 \pi}\right)},
$$

and we have defined a series of new functions though digamma function $\psi(z)$,

$\psi_{\Delta-i \frac{\beta \omega}{2 \pi}}^{(n)} \equiv \psi^{(n)}\left(\Delta-\frac{i \beta \omega}{2 \pi}\right)-\psi^{(n)}\left(1-\Delta-\frac{i \beta \omega}{2 \pi}\right)$,

where $\psi^{(n)}(z)=d^{n} \psi(z) / d z^{n}$ is the $n$-th derivative of the digamma function $\psi(z)=\psi^{(0)}(z)=\Gamma^{\prime}(z) / \Gamma(z)$ which satisfy the reflection formula, $\psi(1-z)-\psi(z)=\pi \cot (\pi z)$.

At low frequency limit $(\omega \ll 1)$, or large temperature limit $(\beta \ll 1)$, the leading-order behavior of the function

$$
\psi_{\Delta-i \frac{i \omega}{2 \pi}}^{(0,1)}=\left\{-\pi \cot (\pi \Delta), \psi^{(1)}(\Delta)-\psi^{(1)}(1-\Delta)\right\}+O(\omega \beta),
$$

which are constants and independent of frequency. If one shifted $\Delta \rightarrow \Delta+1$, then

$$
\begin{aligned}
I_{\Delta+1-\frac{i \beta \omega}{2 \pi}}^{(0,1,2)} & \equiv \int_{0}^{+\infty} \frac{(2 \pi / \beta) d t e^{i \omega t}}{\left[\frac{\beta}{2 \pi} \sinh \frac{t 2 \pi}{2} \beta\right]^{2 \Delta+2}}\left\{1, \frac{2 \pi}{\beta} t,\left(\frac{2 \pi}{\beta}\right)^{2} t^{2}\right\} \\
& =I_{\Delta+1-\frac{i \beta \omega}{2 \pi}}^{(0)}\left\{1,-\psi_{\Delta+1-i \frac{\beta \omega}{2 \pi}}^{(0)}, \psi_{\Delta+1-i \frac{\beta \omega}{2 \pi}}^{(1)}+\left(\psi_{\Delta+1-i \frac{\beta \omega}{2 \pi}}^{(0)}\right)^{2}\right\}, \\
\Delta & <\{-1 / 2,0,1 / 2\}, \quad \beta>0, \quad \operatorname{Im} \omega>0 .
\end{aligned}
$$

For $\Delta \in(0,1 / 2)$ case, one has $I_{\Delta+1-\frac{i \beta \omega}{2 \pi}}^{(0,1)}=0$, but $I_{\Delta+1-\frac{i \beta \omega}{2 \pi}}^{(2)} \neq 0$. It is intuitive to observe that the high-point integral can be obtained through

$$
\begin{aligned}
I_{\Delta+1-\frac{i \beta \omega}{2 \pi}}^{(n)} & \equiv \int_{0}^{+\infty} \frac{(2 \pi / \beta) d t e^{i \omega t}}{\left[\frac{\beta}{2 \pi} \sinh \frac{t}{2} \frac{2 \pi}{\beta}\right]^{2 \Delta+2}}\left(\frac{2 \pi}{\beta}\right)^{n} t^{n} \\
& =\left(\frac{2 \pi}{\beta}\right)^{n} \frac{1}{i^{n}} \frac{d^{n}}{d \omega^{n}} I_{\Delta+1-\frac{i \beta \omega}{2 \pi}}^{(0)},
\end{aligned}
$$

where $n \geq 3$. As would be expected, the higher-order derivatives to the digamma function, i.e., $\psi_{\Delta-i \beta \omega /(2 \pi)}^{(n)}$ will be present. Similarly, one also obtains another series of nonvanishing integrals as

$$
\begin{gathered}
\int_{0}^{+\infty} \frac{2 \pi}{\beta} d t \frac{e^{i \omega t} \cosh \left(\frac{t}{2} \frac{2 \pi}{\beta}\right)}{\left[\frac{\beta}{2 \pi} \sinh \left(\frac{t}{2} \frac{2 \pi}{\beta}\right)\right]^{2 \Delta+1}}\left\{1, \frac{2 \pi}{\beta} t,\left(\frac{2 \pi}{\beta}\right)^{2} t^{2}\right\} \\
=\frac{i \omega}{\Delta} I_{\Delta-\frac{i \beta \omega}{2 \pi}}^{(0)}\left\{1,-\frac{i}{\omega} \frac{2 \pi}{\beta}-\psi_{\Delta-i \frac{\beta \omega}{2 \pi}}^{(0)},\right. \\
\left.+\frac{2 i}{\omega} \frac{2 \pi}{\beta} \psi_{\Delta-i \frac{\beta \omega}{2 \pi}}^{(0)}+\left(\psi_{\Delta-i \frac{\beta \omega}{2 \pi}}^{(0)}\right)^{2}+\psi_{\Delta-i \frac{\beta \omega}{2 \pi}}^{(1)}\right\} . \\
\Delta<\{0,1 / 2,1 / 2\}, \quad \beta>0, \quad \operatorname{Im} \omega>0 .
\end{gathered}
$$

To simplify Eq. (D13), we have also used the relation that

$$
\psi^{(n)}(-x)-\psi^{(n)}(1-x)=\frac{n !}{x^{n+1}},
$$

as well as the relation as below

$$
\psi_{\Delta-i \frac{\beta \omega}{2 \pi}}^{(0)}=H_{\Delta-1-\frac{i \beta \omega}{2 \pi}}-H_{-\Delta-\frac{i \beta \omega}{2 \pi}},
$$

where $H_{n}=\sum_{i=1}^{n} 1 / i$ is the $n$-th harmonic number. This is due to the identity $H_{z}=\psi(z+1)+\gamma_{E}$, where $\gamma_{E} \approx$ 0.577216 is Euler-Mascheroni constant. In low temperature limit $(\beta \gg 1)$, or large frequency limit $(\omega \gg 1)$, one has

$H_{\Delta-\frac{i \beta \omega}{2 \pi}}=\gamma_{E}+\ln \left(-\frac{i \beta \omega}{2 \pi}\right)+\frac{i(2 \Delta+1) \pi}{\beta \omega}+O\left(\beta^{-2}\right)$.

In the low frequency limit $(\omega \ll 1)$ (but with finite temperature), one has

$$
H_{\Delta-\frac{i \beta \omega}{2 \pi}} \stackrel{\omega \rightarrow \infty}{=} H_{\Delta}-\frac{i \beta \omega \psi^{(1)}(\Delta+1)}{2 \pi}+O\left(\omega^{2}\right),
$$


take $\Delta= \pm 1 / 2$ and $\Delta= \pm 1 / 4$ as an examples, one has

$$
\begin{aligned}
H_{\frac{1}{2}-\frac{i \beta \omega}{2 \pi}} & =2-\ln 4-\frac{1}{4} i \pi \beta \omega+\frac{2 i \beta \omega}{\pi}+O\left(\omega^{2}\right), \\
H_{-\frac{1}{2}-\frac{i \beta \omega}{2 \pi}} & =-\ln (4)-\frac{1}{4} i \pi \beta \omega+O\left(\omega^{2}\right), \\
H_{\frac{1}{4}-\frac{i \beta \omega}{2 \pi}} & =-\frac{\pi}{2}+4-\ln (8)-\frac{i \beta \omega}{2 \pi}\left(8 C-16+\pi^{2}\right)+O\left(\omega^{2}\right), \\
H_{-\frac{1}{4}-\frac{i \beta \omega}{2 \pi}} & =\frac{\pi}{2}-\ln (8)-\frac{i \beta \omega}{2 \pi}\left(\pi^{2}-8 C\right)+O\left(\omega^{2}\right),
\end{aligned}
$$

where $C \equiv \sum_{n=0}^{\infty}(-1)^{n} /(2 n+1)^{2} \approx 0.915966$ is Catalan's constant.

[1] A. Kitaev, Talks at KITP, April 7, 2015 and May 27, 2015.

[2] S. Sachdev and J. Ye, Gapless Spin Fluid Ground State in a Random, Quantum Heisenberg Magnet, Phys. Rev. Lett. 70, 3339 (1993).

[3] J. Polchinski and V. Rosenhaus, The spectrum in the Sachdev-Ye-Kitaev model, J. High Energy Phys. 04 (2016) 001.

[4] A. Jevicki, K. Suzuki, and J. Yoon, Bi-Local Holography in the SYK Model, J. High Energy Phys. 07 (2016) 007.

[5] J. Maldacena and D. Stanford, Remarks on the SachdevYe-Kitaev model, Phys. Rev. D 94, 106002 (2016).

[6] K. Jensen, Chaos in $\mathrm{AdS}_{2}$ Holography, Phys. Rev. Lett. 117, 111601 (2016).

[7] J. Maldacena, D. Stanford, and Z. Yang, Conformal symmetry and its breaking in two dimensional Nearly Antide-Sitter space, Prog. Theor. Exp. Phys. 2016, $12 \mathrm{C} 104$ (2016).

[8] J. Engelsöy, T. G. Mertens, and H. Verlinde, An investigation of $\mathrm{AdS}_{2}$ backreaction and holography, J. High Energy Phys. 07 (2016) 139.

[9] D. Bagrets, A. Altland, and A. Kamenev, Sachdev-YeKitaev model as Liouville quantum mechanics, Nucl. Phys. B911, 191 (2016).

[10] M. Cvetič and I. Papadimitriou, $\mathrm{AdS}_{2}$ holographic dictionary, J. High Energy Phys. 12 (2016) 008; Erratum, J. High Energy Phys. 01 (2017) 120(E).

[11] Y. Gu, X. L. Qi, and D. Stanford, Local criticality, diffusion and chaos in generalized Sachdev-Ye-Kitaev models, J. High Energy Phys. 05 (2017) 125.

[12] D. J. Gross and V. Rosenhaus, A Generalization of SachdevYe-Kitaev, J. High Energy Phys. 02 (2017) 093.

[13] M. Berkooz, P. Narayan, M. Rozali, and J. Simón, Higher Dimensional Generalizations of the SYK Model, J. High Energy Phys. 01 (2017) 138.

[14] E. Witten, An SYK-Like Model Without Disorder, arXiv:1610.09758.

[15] J. S. Cotler, G. Gur-Ari, M. Hanada, J. Polchinski, P. Saad, S. H. Shenker, D. Stanford, A. Streicher, and M. Tezuka, Black holes and random matrices, J. High Energy Phys. 05 (2017) 118.
[16] R. A. Davison, W. Fu, A. Georges, Y. Gu, K. Jensen, and S. Sachdev, Thermoelectric transport in disordered metals without quasiparticles: The Sachdev-Ye-Kitaev models and holography, Phys. Rev. B 95, 155131 (2017).

[17] G. Turiaci and H. Verlinde, Towards a 2d QFT Analog of the SYK Model, J. High Energy Phys. 10 (2017) 167.

[18] A. M. Garcia-Garcia and J. J. M. Verbaarschot, Analytical spectral density of the Sachdev-Ye-Kitaev model at finite $N$, Phys. Rev. D 96, 066012 (2017).

[19] Z. Bi, C. M. Jian, Y.Z. You, K. A. Pawlak, and C. Xu, Instability of the non-Fermi liquid state of the Sachdev-YeKitaev Model, Phys. Rev. B 95, 205105 (2017).

[20] G. Mandal, P. Nayak, and S. R. Wadia, Coadjoint orbit action of Virasoro group and two-dimensional quantum gravity dual to SYK/tensor models, J. High Energy Phys. 11 (2017) 046.

[21] D. J. Gross and V. Rosenhaus, The Bulk Dual of SYK: Cubic Couplings, J. High Energy Phys. 05 (2017) 092.

[22] S. K. Jian and H. Yao, Solvable Sachdev-Ye-Kitaev Models in Higher Dimensions: from Diffusion to Many-Body Localization, Phys. Rev. Lett. 119, 206602 (2017).

[23] D. Stanford and E. Witten, Fermionic localization of the schwarzian theory, J. High Energy Phys. 10 (2017) 008.

[24] C. Peng, Vector models and generalized SYK models, J. High Energy Phys. 05 (2017) 129.

[25] S. R. Das, A. Jevicki, and K. Suzuki, Three dimensional view of the SYK/AdS duality, J. High Energy Phys. 09 (2017) 017.

[26] X. Y. Song, C. M. Jian, and L. Balents, A Strongly Correlated Metal Built from Sachdev-Ye-Kitaev Models, Phys. Rev. Lett. 119, 216601 (2017).

[27] T. G. Mertens, G. J. Turiaci, and H. L. Verlinde, Solving the schwarzian via the conformal bootstrap, J. High Energy Phys. 08 (2017) 136.

[28] Y. Chen, H. Zhai, and P. Zhang, Tunable quantum chaos in the Sachdev-Ye-Kitaev model coupled to a thermal bath, J. High Energy Phys. 07 (2017) 150.

[29] D. J. Gross and V. Rosenhaus, A line of CFTs: from generalized free fields to SYK, J. High Energy Phys. 07 (2017) 086. 
[30] Y. Gu, A. Lucas, and X. L. Qi, Spread of entanglement in a Sachdev-Ye-Kitaev chain, J. High Energy Phys. 09 (2017) 120.

[31] R. G. Cai, S. M. Ruan, R. Q. Yang, and Y. L. Zhang, The String Worldsheet as the Holographic Dual of SYK State, arXiv: 1709.06297.

[32] D. J. Gross and V. Rosenhaus, All point correlation functions in SYK, J. High Energy Phys. 12 (2017) 148.

[33] A. Kitaev and S. J. Suh, The soft mode in the Sachdev-YeKitaev model and its gravity dual, J. High Energy Phys. 05 (2018) 183.

[34] F. M. Haehl and M. Rozali, Fine Grained Chaos in $A d S_{2}$ Gravity, Phys. Rev. Lett. 120, 121601 (2018).

[35] X. Wu, X. Chen, C. M. Jian, Y.Z. You, and C. Xu, Candidate theory for the strange metal phase at a finiteenergy window, Phys. Rev. B 98, 165117 (2018).

[36] A. Gaikwad, L. K. Joshi, G. Mandal, and S. R. Wadia, Holographic dual to charged SYK from 3D Gravity and Chern-Simons, arXiv:1802.07746.

[37] K. S. Kolekar and K. Narayan, $A d S_{2}$ dilaton gravity from reductions of some nonrelativistic theories, Phys. Rev. D 98, 046012 (2018).

[38] O. Parcollet and A. Georges, Non-Fermi-liquid regime of a doped Mott insulator, Phys. Rev. B 59, 5341 (1999).

[39] D. R. Grempel and M. J. Rozenberg, Fluctuations in a Quantum Random Heisenberg Paramagnet, Phys. Rev. Lett. 80, 389 (1998).

[40] A. Georges, O. Parcollet, and S. Sachdev, Mean Field Theory of a Quantum Heisenberg Spin Glass, Phys. Rev. Lett. 85, 840 (2000).

[41] A. Georges, O. Parcollet, and S. Sachdev, Quantum fluctuations of a nearly critical Heisenberg spin glass, Phys. Rev. B 63, 134406 (2001).

[42] C. M. Varma, P. B. Littlewood, S. Schmitt-Rink, E. Abrahams, and A. E. Ruckenstein, Phenomenology of the normal state of $\mathrm{Cu}-\mathrm{O}$ high-temperature superconductors, Phys. Rev. Lett. 63, 1996 (1989).

[43] S. Sachdev, Holographic Metals and the Fractionalized Fermi Liquid, Phys. Rev. Lett. 105, 151602 (2010).

[44] R. Jackiw, Lower dimensional gravity, Nucl. Phys. B252, 343 (1985).

[45] C. Teitelboim, Gravitation and hamiltonian structure in two space-time dimensions, Phys. Lett. 126B, 41 (1983).

[46] A. Almheiri and J. Polchinski, Models of $A d S_{2}$ backreaction and holography, J. High Energy Phys. 11 (2015) 014.

[47] P. Hohenberg and W. Kohn, Inhomogeneous electron gas, Phys. Rev. 136, B864 (1964).

[48] Y. Nomura, S. Sakai, M. Capone, and R. Arita, Unified understanding of superconductivity and Mott transition in alkali-doped fullerides from first principles, Sci. Adv. 1, e1500568 (2015).

[49] A. Georges, G. Kotliar, W. Krauth, and M. J. Rozenberg, Dynamical mean-field theory of strongly correlated fermion systems and the limit of infinite dimensions, Rev. Mod. Phys. 68, 13 (1996).

[50] E. Witten, Anti-de Sitter space and holography, Adv. Theor. Math. Phys. 2, 253 (1998).

[51] W. Mueck and K. S. Viswanathan, Conformal field theory correlators from classical scalar field theory on $\operatorname{AdS}(d+1)$, Phys. Rev. D 58, 041901 (1998).
[52] A. Virosztek and J. Ruvalds, Nested-Fermi-liquid theory, Phys. Rev. B 42, 4064 (1990).

[53] O. Parcollet, A. Georges, G. Kotliar, and A. Sengupta, Overscreened multichannel SU(N) Kondo model: Large-N solution and conformal field theory, Phys. Rev. B 58, 3794 (1998).

[54] D. Chowdhury, Y. Werman, E. Berg, and T. Senthil, Translationally Invariant Non-Fermi Liquid Metals with Critical FermiSurfaces: Solvable Models, Phys. Rev. X 8, 031024 (2018).

[55] S. Sachdev, Bekenstein-Hawking Entropy and Strange Metals, Phys. Rev. X 5, 041025 (2015).

[56] R. G. Cai, Y. H. Qi, Y. L. Wu, and Y. L. Zhang, Topological Non-Fermi liquid, Phys. Rev. D 95, 124026 (2017).

[57] O. Aharony, S. S. Gubser, J. M. Maldacena, H. Ooguri, and Y. Oz, Large $N$ field theories, string theory and gravity, Phys. Rep. 323, 183 (2000).

[58] S. H. Shenker and D. Stanford, Black holes and the butterfly effect, J. High Energy Phys. 03 (2014) 067.

[59] J. Maldacena, S. H. Shenker, and D. Stanford, A bound on chaos, J. High Energy Phys. 08 (2016) 106.

[60] M. Blake, H. Lee, and H. Liu, A quantum hydrodynamical description for scrambling and many-body chaos, J. High Energy Phys. 10 (2018) 127.

[61] A. I. Larkin and Yu. N. Ovchinnikov, Quasiclassical Method in the Theory of Superconductivity, Zh. Eksp. Teor. Fiz. 55, 2262 (1969) [Sov. Phys. J. Exp. Theor. Phys. 28, 1200 (1969)].

[62] D. A. Roberts and D. Stanford, Diagnosing Chaos Using Four-Point Functions in Two-Dimensional Conformal Field Theory, Phys. Rev. Lett. 115, 131603 (2015).

[63] N. Tsuji, P. Werner, and M. Ueda, Exact out-of-time-ordered correlation functions for an interacting lattice fermion model, Phys. Rev. A 95, 011601 (2017).

[64] P. Caputa, Y. Kusuki, T. Takayanagi, and K. Watanabe, Outof-Time-Ordered Correlators in $\left(T^{2}\right)^{n} / \mathbb{Z}_{n}$, Phys. Rev. D 96, 046020 (2017).

[65] D. Z. Freedman, S. D. Mathur, A. Matusis, and L. Rastelli, Correlation functions in the $\operatorname{CFT}(d) / \operatorname{AdS}(d+1)$ correspondence, Nucl. Phys. B546, 96 (1999).

[66] J. S. Schwinger, Brownian motion of a quantum oscillator, J. Math. Phys. (N.Y.) 2, 407 (1961).

[67] L. V. Keldysh, Diagram technique for nonequilibrium processes, Zh. Eksp. Teor. Fiz. 47, 1515 (1964) [Sov. Phys. J. Exp. Theor. Phys. 20, 1018 (1965)].

[68] E. Witten, Coadjoint orbits of the Virasoro Group, Commun. Math. Phys. 114, 1 (1988).

[69] A. Alekseev and S. L. Shatashvili, Path integral quantization of the coadjoint orbits of the virasoro group and $2 \mathrm{~d}$ gravity, Nucl. Phys. B323, 719 (1989).

[70] E. M. Lifshitz and L. P. Pitaevskii, Statistical Physics, Part 2: Theory of the Condensed State (Pergamon Press, Oxford, 1980).

[71] A. Strominger, $\operatorname{AdS}(2)$ quantum gravity and string theory, J. High Energy Phys. 01 (1999) 007.

[72] V. de Alfaro, S. Fubini, and G. Furlan, Conformal invariance in quantum mechanics, Nuovo Cimento A 34, 569 (1976).

[73] C. Chamon, R. Jackiw, S. Y. Pi, and L. Santos, Conformal quantum mechanics as the $\mathrm{CFT}_{1}$ dual to $\mathrm{AdS}_{2}$, Phys. Lett. B 701, 503 (2011).

[74] R. Jackiw and S.-Y. Pi, Conformal blocks for the 4-Point function in conformal quantum mechanics, Phys. Rev. D 86, 
045017 (2012); Erratum, Phys. Rev. D 86, 089905(E) (2012).

[75] T. Faulkner, H. Liu, J. McGreevy, and D. Vegh, Emergent quantum criticality, Fermi surfaces, and $\operatorname{AdS}(2)$, Phys. Rev. D 83, 125002 (2011).

[76] T. Faulkner, N. Iqbal, H. Liu, J. McGreevy, and D. Vegh, Holographic non-Fermi liquid fixed points, Phil. Trans. R. Soc. A 369, 1640 (2011).

[77] A. A. Abrikosov, L. P. Gorkov, and I. E. Dzyaloshinski, Methods of Quantum Field Theory in Statistical Physics,
Revised ed., edited by R. A. Silverman (Dover, New York, 1963).

[78] S. Weinberg, The Quantum Theory of Fields. Vol. 1: Foundations (Cambridge University Press, Cambridge, England, 1995).

[79] I. S. Gradshteyn and I. M. Ryzhik, Table of Integrals, Series and Products (Academic Press, New York, 2007).

[80] A. A. Patel, J. McGreevy, D. P. Arovas, and S. Sachdev, Magnetotransport in a Model Of A Disordered Strange Metal, Phys. Rev. X 8, 021049 (2018). 